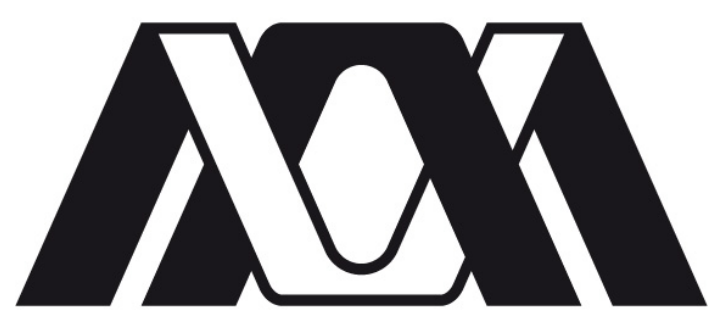

UNIVERSIDAD AUTÓNOMA METROPOLITANA

Unidad Iztapalapa

División de Ciencias Básicas e Ingeniería

\title{
CICLO DE STIRLING PARA UNA PARTÍCULA BROWNIANA
}

Tesis presentada para obtener el grado de Maestra en Ciencias (Física) por:

Fís. Ana Elisa Zamudio Murillo

Asesora de tesis:

Dra. Rosa María Velasco Belmont

Sinodales:

Presidente: Dra. Rosa María Velasco Belmont

Secretario: Dr. José Inés Jiménez Aquino

Vocal: Dra. Lorena Romero Salazar

14 de Noviembre del 2019

EP-001 UAM-Iztapalapa 


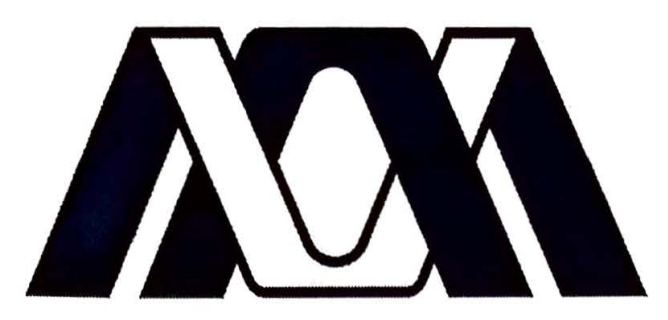

\author{
UNIVERSIDAD AUTÓNOMA METROPOLITANA \\ Unidad Iztapalapa \\ División de Ciencias Básicas e Ingeniería
}

\title{
CICLO DE STIRLING PARA UNA PARTÍCULA BROWNIANA
}

Tesis presentada para obtener el grado de Maestra en Ciencias (Física) por:

Fís. Ana Elisa Zamudio Murillo

Asesora de tesis:

Dra. Rosa María Velasco Belmont
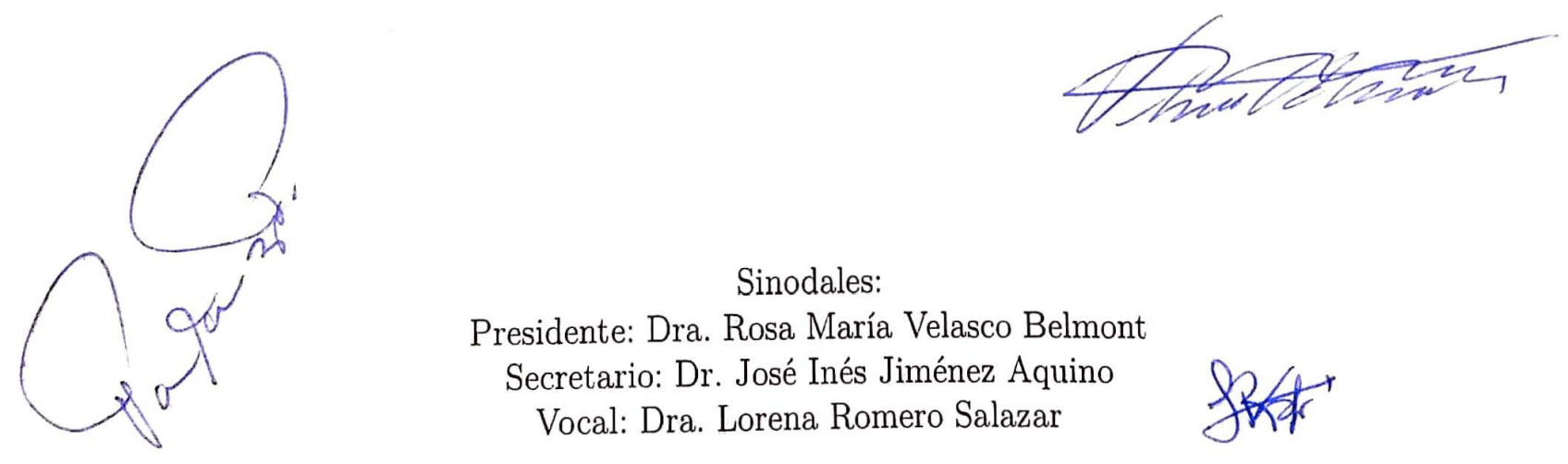

Sinodales:

Presidente: Dra. Rosa María Velasco Belmont Secretario: Dr. José Inés Jiménez Aquino Vocal: Dra. Lorena Romero Salazar

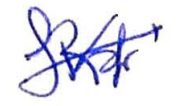




\section{Agradecimientos}

Agradezco el apoyo de CONACYT sin el cual no pude haberme dedicado completamente a mi maestría. A mi asesora, a la que admiro en todo sentido, que me ha guiado con paciencia y entusiasmo. Agradezco a mis papás por siempre apoyarme incondicionalmente, por la ardua revisión de esta tesis y sus porras. A Gil por ser mi motor en todo momento. A Nahum por enseñarme sobre las pinzas ópticas, mostrármelas experimentalmente y de forma teórica y además de todo eso por prestarme las ilustraciones que realizó en su tesis. Finalmente, a todos mis amigos que hicieron de esta experiencia la mejor. 



\section{Resumen}

La termodinámica estocástica estudia la termodinámica de sistemas pequeños en los cuales las fluctuaciones del medio afectan al sistema. Experimentalmente se han logrado realizar máquinas térmicas que completan ciclos de Carnot y Stirling con una molécula Browniana. Estas máquinas, debido a que se ven afectadas por las fluctuaciones, tienen un trabajo y entropía diferente al caso macroscópico. En nuestro modelo se resuelve la ecuación de Fokker Planck para el caso sobre amortiguado en presencia de un potencial tipo oscilador armónico el cual varía en el tiempo de acuerdo al protocolo que se aplica para completar un ciclo de Stirling, la termodinámica estocástica nos permite realizar un análisis de la entropía, el trabajo, y la eficiencia del ciclo de manera que se puede cuantificar qué tanto se ve afectado el ciclo por las fluctuaciones del medio a las que se encuentra sujeta la partícula. 



\section{Índice general}

\begin{tabular}{lll}
\hline Lista de figuras & IX
\end{tabular}

\begin{tabular}{lll}
\hline Lista de tablas & XI \\
\hline
\end{tabular}

$\begin{array}{ll}\text { 1. Introducción } & 1\end{array}$

$\begin{array}{ll}\text { 2. Relación Experimental } & 9\end{array}$

2.1. Pinzas ópticas . . . . . . . . . . . . . . . . . . . 9 9

2.1.1. Pinzas ópticas de un solo haz . . . . . . . . . . . . . . . . 11

2.2. Modelo experimental . . . . . . . . . . . . . . . . . 13

$\begin{array}{ll}\text { 3. Termodinámica estocástica } & 17\end{array}$

3.1. Primera ley de la termodinámica . . . . . . . . . . . . . . . . 20

3.1.1. Calor. . . . . . . . . . . . . . . . . . 21

3.1.2. Trabajo . . . . . . . . . . . . . . . . . 22

3.2. Entropía . . . . . . . . . . . . . . . . . . . . . 24

4. Ecuación de Langevin y Fokker Planck 25

4.1. Ecuación de Langevin para el oscilador ar-

mónico ......................... 25

4.2. Ecuación de Fokker-Planck . . . . . . . . . . . . . . . . . . 27

4.2.1. Relación entre la ecuación de Langevin y la ecuación de Fokker-Planck . . . . . . . . . . . . . . . . . . . 28

4.3. El oscilador armónico con dependencia temporal . . . . . . . . . . 29

5. Ciclo de Stirling 33

5.1. Ciclo de Stirling . . . . . . . . . . . . . . . . . . . . . . 33

5.2. Ciclo de Stirling en cuasi-equilibrio . . . . . . . . . . . . . 36

$\begin{array}{ll}\text { 6. Ciclo fuera de equilibrio } & 43\end{array}$

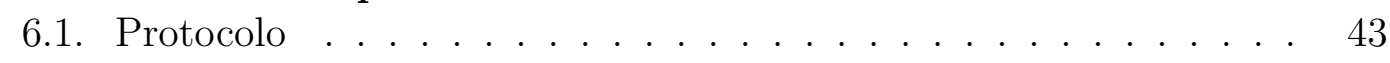

6.2. Ecuación de Langevin en el ciclo . . . . . . . . . . . . . . . . . . . . . 4 45

6.3. Ecuación de Fokker-Planck en el ciclo . . . . . . . . . . . . . . . . 49

\begin{tabular}{ll}
\hline 7. Termodinámica del ciclo & 55
\end{tabular}

7.0.1. Calor . . . . . . . . . . . . . . . . . . 55

7.0 .2$. Trabajo . . . . . . . . . . . . . 56

7.0.3. Entropía . . . . . . . . . . . . . . . . . . . . 57

7.1. Disminución del potencial . . . . . . . . . . . . . 58 
7.2. Disminución de la intensidad del ruido . . . . . . . . . . . . . . 62

7.3. Aumento del potencial . . . . . . . . . . . . . . . 63

7.4. Aumento de la intensidad del ruido . . . . . . . . . . . . . . . . . 66

7.5. Análisis ........................ 69

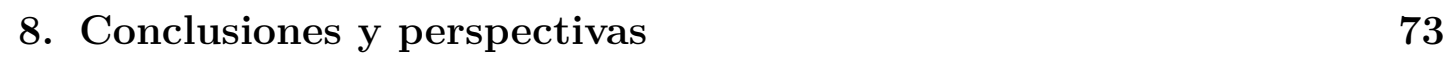

\begin{tabular}{ll}
\hline A. Análisis dimensional & 77
\end{tabular}

\begin{tabular}{ll}
\hline B. Solución a la ecuación de Langevin & 79
\end{tabular}

\begin{tabular}{ll}
\hline C. Solución a Fokker-Planck & 87
\end{tabular}

C.1. Proceso de 1 a 2 . . . . . . . . . . . . . . . . . 87

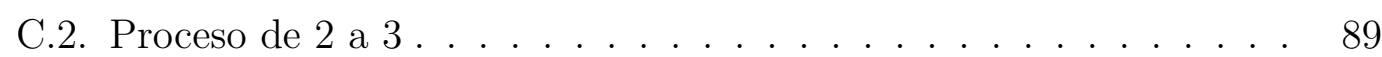

C.3. Proceso de 3 a 4 . . . . . . . . . . . . . . . . . . 91

C.4. Proceso de 4 a $1 . \ldots . \ldots . \ldots . \ldots 92$

\begin{tabular}{lr}
\hline D. Cálculos del calor & 95
\end{tabular}

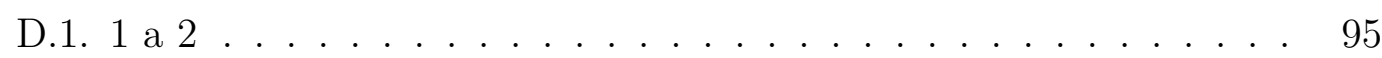

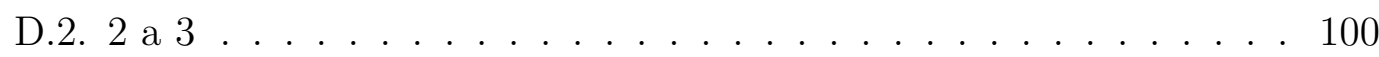

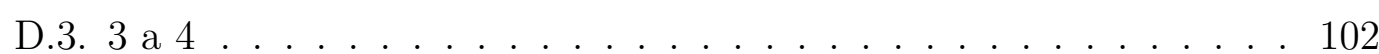

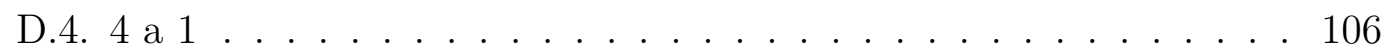

\begin{tabular}{ll}
\hline E. Trabajo en el ciclo & 109
\end{tabular}

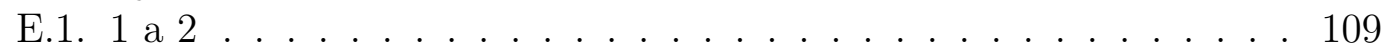

E.2.2 a $3 \ldots \ldots \ldots \ldots 111$

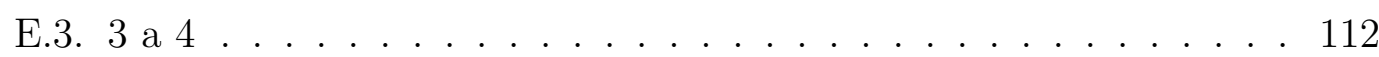

E.4.4a $1 \ldots \ldots \ldots \ldots \ldots \ldots \ldots$

\begin{tabular}{lr}
\hline F. Entropía en el ciclo & 115
\end{tabular}

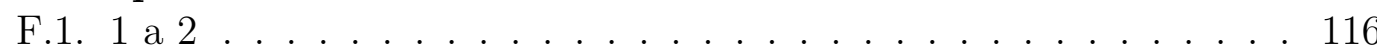

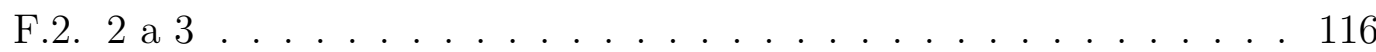

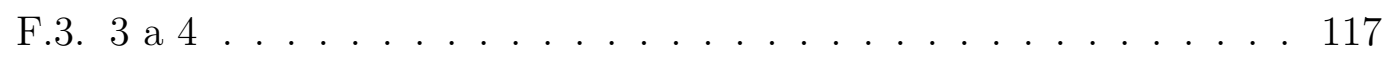

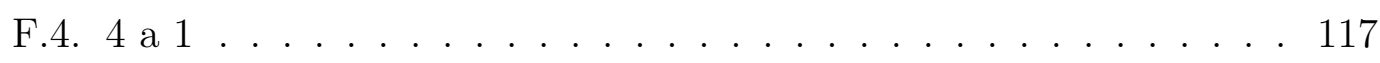

F.4.1. Análisis ciclo completo . . . . . . . . . . . . . 118 


\section{Índice de figuras}

1.1. Ejemplo de una trayectoria irregular que sigue una partícula Browniana. 6

1.2. Se muestran tres dibujos registrados en el artículo de Perrin ${ }^{24}$ obtenidos al trazar los segmentos que unen las posiciones consecutivas del mismo grano en intervalos de 30 segundos. . . . . . . . . . . . . . . . . . . . . 4

1.3. Tabla con una de las muchas medidas realizadas por Chaudesaigues. En este caso se realizó para 50 granos de radio $0.212 \mu m$ inmersos en un fluido con una viscosidad de $0.012 \mathrm{~Pa} \cdot \mathrm{s}$ a una temperatura $\mathrm{T}=17^{\circ} \mathrm{C}$. Las medidas se tomaron cada 30 segundos. En la tabla se muestra el desplazamiento promedio horizontal, $\xi$ es el coeficiente de difusión dado por Einstein y el valor del número de Avogadro que obtuvieron |24] . . . 5

2.1. De la referencia (22) se muestra el diagrama ilustrativo de las fuerzas que actúan sobre la partícula generadas por un haz plano, cuando cambia el medio por que el pasa el haz, la fuerza $F_{a}$ es generada por el rayo a y $F_{b}$ es generado por el rayo b y la fuerza de dispersión $F_{\text {scat }}$ es la fuerza generada por la presión de radiación. . . . . . . . . . . . . . . 10

2.2. Diagrama de las direcciones de las fuerzas que son generadas con un haz Gaussiano.22 . . . . . . . . . . . . . . . . . . . . . . . . . . . . 11

2.3. Modelo de la primera primera trampa óptica realizada con dos haces de luz Gaussianos, la fuerza gradiente va en dirección del máximo del haz!22 11

2.4. Se muestra el efecto del la fuerza de dispersión y gradiente sobre una esfera, las cuales generan el atrapamiento cuando se está usando un lente objetivo.22] . . . . . . . . . . . . . . . . . . . . . . . . . . 12

2.5. Diagrama de las fuerzas sobre la partícula cuando su centro se encuentra adelante o atrás del punto focal.22 . . . . . . . . . . . . . . . . . . . 13

2.6. Potencial generado por las pinzas ópticas visto como un resorte de Hooke lineal.39 . . . . . . . . . . . . . . . . . . . . . . . . 13

2.7. Arreglo experimental de pinzas ópticas con dos potenciales externos, $V_{k}$ conectado al láser y $V_{q}$ conectado a la cámara donde se encuentra la partícula.21 . . . . . . . . . . . . . . . . . . . . . 14

4.1. Del lado izquiero en la figura a se muestra la gráfica puramente cualitativa del comportamiento del promedio de la posición de la partícula, del lado derecho en la figura b se muestra la gráfica de la correlación de la posición. . . . . . . . . . . . . . . . . . . . . . . . . . . . 27

5.1. Ciclo de Stirling que consiste de cuatro procesos, la compresión y expansión isotérmica, y a volumen constante la adición y sustracción de calor. . . . . . . . . . . . . . . . . . . . . . . . 34 
5.2. Arreglo de pistones de la máquina ${ }^{38}$. . . . . . . . . . . . . . . 35

5.3. Se muestra de manera gráfica el cambio que se genera cuando se varía la rigidez del potencial con $k_{2}=2 k_{1}$. . . . . . . . . . . . . . . . . 36

5.4. Cilo de Stirling Browniano en el espacio $\Phi, k$ en donde las curvas del espacio se generan a partir de la ecuación de estado 5.20 . . . . . . . . . 40

6.1. Gráficas realizadas con el protocolo arriba indicado. En la figura derecha se muestra el protocolo para el cambio en la intensidad del ruido $q(t)$ en función del tiempo $t$, en donde $q_{2}=2 q_{1}$. En la figura izquierda se muestra el protocolo para el cambio de la rigidez del potencial $k(t)$ en función del tiempo t, en el cual se toma en cuenta que $k_{2}=2 k_{1}$. . . . 45

6.2. La posición de la patícula y su promedio durante la disminución del potencial . . . . . . . . . . . . . . . . . . 47

6.3. Gráfica de la posición durante el segundo proceso del ciclo en el que la intensidad del ruido cambia con una rigidez del potencial constante. . . 48

6.4. Gráfica para el tercer proceso del ciclo, en donde se muestra la posición de la partícula Browniana y su promedio. . . . . . . . . . . . . . . . . 49

6.5. Último proceso del ciclo. Gráfica de la posición de una partícula Browniana durante este proceso. . . . . . . . . . . . . . . . . . . 50

7.1. Gráfica del calor adimensional durante el primer proceso en función de a para $\Delta k=1$ y $\Delta q=1 \ldots \ldots \ldots \ldots \ldots$. . . . . . . . . . 59

7.2. Gráfica del trabajo adimensional durante el primer proceso en función de $\alpha$ para $\Delta k=1$ y $\Delta q=1 \ldots \ldots \ldots \ldots$. . . . . . . . . 60

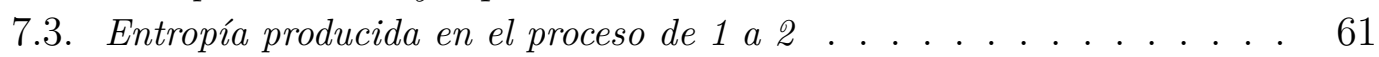

7.4. Calor durante el segundo proceso del ciclo, para $\Delta k=1$ y $\Delta q=1$. . . 62

7.5. Entropía durante el proceso de 2 a 3. . . . . . . . . . . . . . 64

7.6. Calor durante el aumento del potencial(Proceso de 3 a 4). . . . . . . . . 65

7.7. Gráfica del trabajo durante el proceso 3 a 4 con $\Delta k=1$ y $\Delta q=1$. . . 66

7.8. Cambio de entropía durante el proceso de 3 a 4 . . . . . . . . . . . . . 67

7.9. Calor durante el proceso de 4 a 1 . . . . . . . . . . . . . . . . . . . 67

7.10. Cambio en la entropía durante el proceso de 4 a 1 . . . . . . . . . . . . . 68

7.11. Gráfica del calor durante todo el ciclo, la línea naranja corresponde a la disminución del potencial, la negra corresponde a la disminución de la intensidad del ruido, la azul al aumento del potencial y la roja concierne al aumento de la intensidad del ruido. . . . . . . . . . . . . . . . . . 69

7.12. Gráfica del trabajo durante todo el ciclo . . . . . . . . . . . . . . . . . . 70

7.13. Cambio en la entropía durante el ciclo. . . . . . . . . . . . . . . . 71

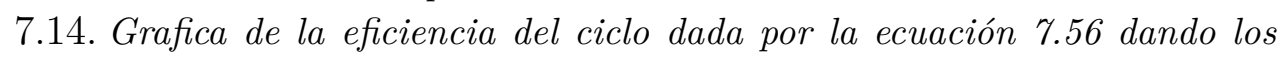
valores numéricos a las variables adimensionales $\Delta k=1$ y $\Delta q=1$. . . 72 


\section{Índice de cuadros}

2.1. Tabla con los valores numéricos del experimento realizado por Martínez. ${ }^{21} 15$

5.1. Tabla con los valores del calor y el trabajo en cada uno de los procesos del ciclo de Stirling. . . . . . . . . . . . . . . . . . 41 



\section{Capítulo 1}

\section{Introducción}

La necesidad filosófica de comprender el mundo que nos rodea nos ha llevado a pensar en lo pequeño; comenzó con la convicción de Demócrito de que dividiendo una gota de agua en partes se obtendrían cada vez gotas más pequeñas. Pero, ¿qué pasaría si llegase un punto en el que fuera imposible continuar con la división? Este pensamiento de conocer lo pequeño nos ha llevado por una senda de diversos estudios y nos hemos topado con varias sorpresas. Tan sólo en el mundo micrométrico se han descubierto propiedades diferentes y únicas que se observan por el microscopio y en algunos casos se pueden llegar a observar a simple vista. Estas propiedades emergen en sistemas inertes como el polvo, biomoléculas como las proteínas y hasta seres vivos como las bacterias; y son características del movimiento Browniano.

Edward Nelson ${ }^{23}$ en su libro nos relata la historia del hallazgo del movimiento Browniano y sus investigaciones. El movimiento Browniano se define como un movimiento azaroso de partículas muy pequeñas que se encuentran en un fluido. El descubrimiento de este movimiento se le atribuye a un botánico inglés llamado Robert Brown, en 1828. Se le atribuyó a él, no por ser el primero en observarlo puesto que ya varios científicos lo habían hecho, y el mismo Brown menciona en su artículo ${ }^{7}$ a sus precursores: Leeuwenhoek, Buffon y Spallanzani en el siglo XVI. Sin embargo, fue Brown el primero en realizar estudios detallados sobre el fenómeno, relatando en ellos todo el proceso de su descubrimiento.

Según se relata,,$\sqrt[6]{23}$ al observar los granos de polen bajo el microscopio en una solución acuosa notó que tenían un movimiento aleatorio, tal como se muestra en la figura 1.1. Pensando que quizás este movimiento se debía a la especie de polen, se dedicó a observar pólenes de diferentes especies obteniendo resultados similares. Su primera hipótesis fue que el movimiento Browniano no solo era vital (de partículas con vida) sino peculiar de las células sexuales masculinas de las plantas, cosa que él mismo refutó posteriormente. Estudió muchos tipos de sustancias orgánicas, encontrando en todas ellas el mismo tipo de movimiento, luego estudió los minerales, llegando a la conclusión de que el movimiento no estaba limitado a los cuerpos orgánicos.

Una de sus principales aportaciones al estudio del movimiento fue el idear un experimento con el que se logró refutar todas las teorías que explicaban que el 


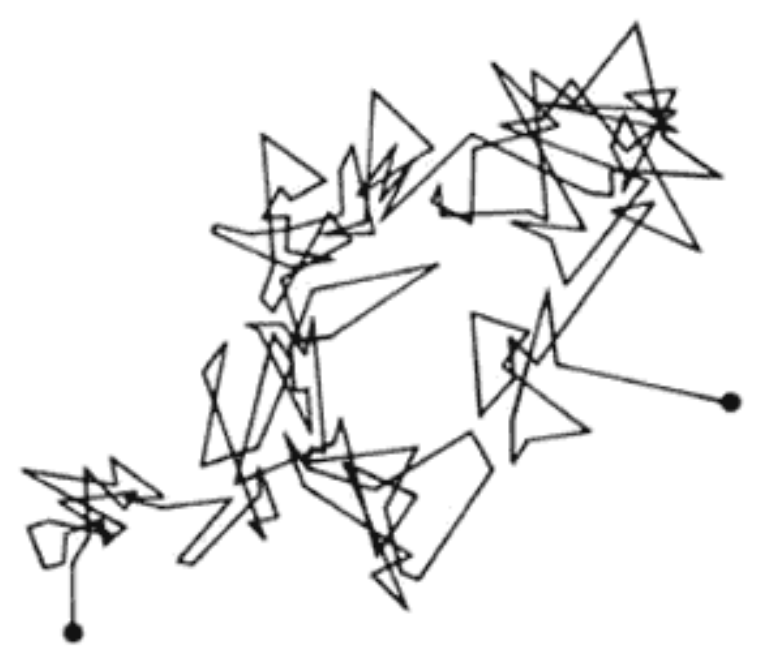

Figura 1.1: Ejemplo de una trayectoria irregular que sigue una partícula Browniana!

movimiento Browniano era generado por fuerzas externas, tales como: la evaporación, burbujas diminutas de aire, un equilibrio inestable en el fluido en el que se encuentra la partícula o fuerzas como la atracción y repulsión entre las partículas mismas. El experimento consistía en sumergir una gota de agua, que contenía una partícula micrométrica, en aceite. De manera que el agua se encontraba aislada de cualquier perturbación externa a través del aceite; al observar la partícula se encontró con un movimiento aparentemente inagotable. Pero entonces ¿cuál era el motivo del movimiento? A pesar de las arduas investigaciones de Brown sobre el movimiento Browniano, él no expresó una conclusión al respecto, describió como teoría que la materia que se encontraba estudiando a las que llamó moléculas activas mostraban un movimiento rápido e irregular cuyo origen provenía de las mismas partículas y no del medio que las rodeaba. Demostró que no tenía un origen mecánico y que era posible encontrar dicho movimiento tanto en partículas orgánicas como inorgánicas.

Posterior a la ardua investigación de Brown, una gran cantidad de científicos trataron de darle una explicación al fenómeno. Wiener en 1863, formuló varios argumentos para mostrar que el movimiento Browniano no podía atribuirse a causas externas, sino que tenía que deberse a movimientos internos del fluido. Perrin,$\sqrt[24]{,}$ dio una interpretación probabilística muy interesante en la cual explica que las colisiones moleculares del líquido, las cuales causan presión, no actúan uniformemente sobre la partícula Browniana ya que la superficie tiene un área tan pequeña que no puede compensar las irregularidades de la presión que ejercen las moléculas del líquido y mientras más pequeña sea la partícula Browniana se notarán más las desigualdades de la presión y en consecuencia se observarán oscilaciones cada vez más enérgicas.

Surgieron muchas más hipótesis sobre el origen del fenómeno, sin embargo, no se lograba llegar a un consenso. Jevons decía que el movimiento tenía un origen eléctrico, Ord atribuía este movimiento a las vibraciones de los coloides. Guoy en 1888 encontró que el movimiento era más intenso cuando la viscosidad del disolvente era menor, comprobó argumentos que soportaban la teoría cinética, basada en la suposición atómica y relacionó el movimiento con la agitación térmica 
molecular del líquido.

Nelson resume esta ardua investigación realizada por los científicos que hemos mencionado y otros más sobre el movimiento Browniano en siete puntos importantes. .23

1. El movimiento irregular de la partícula Browniana, se compone de traslaciones y rotaciones, en estos movimientos parece no haber tangente. (Perrin mencionó la no diferenciabilidad de las curvas).

2. Dos partículas parecen moverse independientemente, incluso cuando la distancia de separación entre ellas es menor que la longitud de su diámetro (Este hecho fue observado por Brown).

3. Al reducir el tamaño de la partícula, el movimiento es más activo.

4. La composición y la densidad de la partícula no tienen efecto sobre el movimiento.

5. En fluidos menos viscosos el movimiento es más activo.

6. Cuando la temperatura aumenta el movimiento resulta más activo. (El efecto de la viscosidad predomina sobre la temperatura).

7. El movimiento nunca para. (Cantoni y Oehl en $1865^{6}$ realizaron un experimento en el cual mantuvieron sellada herméticamente una suspensión durante un año y encontraron que el movimiento Browniano de las partículas no se alteró en ese intervalo de tiempo.)

Hasta ese momento todos los argumentos sobre el movimiento Browniano habían sido cualitativos. Unos años después, Albert Einstein publicó en 1905 un trabajo en el que propuso la explicación al movimiento Browniano. De hecho, predijo el movimiento sin un conocimiento completo de todo el trabajo que ya se había realizado, como él mismo lo menciona en su artículo! 10

"It is possible that the movements to be discussed here are identical with the socalled 'Brownian molecular motion'; however, the information available to me regarding the latter is so lacking in precision, that I can form no judgment in the matter."

En su trabajo predijo la relación del movimiento de las partículas suspendidas con la difusión y su desplazamiento cuadrático medio, dando una explicación del origen del movimiento. En un tiempo $t_{c o l}$, un tiempo extremadamente corto, la partícula Browniana se comporta como una partícula libre, esto implica que no es perturbada por choques. Este tiempo es muy pequeño para ser comparado con observables macroscópicas pero su magnitud es tal que el movimiento que ejecuta una partícula en dos intervalos consecutivos de tiempo $t_{c o l}$ pueden considerarse fenómenos mutuamente independientes. Al hacer la aproximación a tiempos $t$ grandes comparados con $t_{c o l}$ la partícula Browniana comienza a chocar con las partículas del medio en el que se encuentra, este movimiento corresponde al régimen difusivo de la partícula en el fluido, en este régimen, la raíz cuadrada del promedio aritmético del cuadrado del desplazamiento que tiene una partícula 
Browniana en un tiempo $t$ puede ser determinado y está dado por:

$$
\lambda=\sqrt{x^{2}}=\sqrt{2 D t}
$$

en donde $D$, el coeficiente de difusión para un material suspendido en un líquido, depende de varias cantidades como la temperatura del fluido $(T)$, el coeficiente de viscosidad $(\eta)$, las dimensiones de la partícula ( $a$ el radio), $R$ la constante universal de los gases ideales y el número de Avogadro $(N)$

$$
D=\frac{R T}{N 6 \pi \eta a} \text {. }
$$

Los argumentos de Einstein nos indican la naturaleza del movimiento sin darnos una teoría dinámica y gracias a ello se logra explicar el por qué mientras más pequeña es la partícula Browniana mayor es el movimiento y nunca para. Cuando disminuimos el radio de la partícula en (1.2), el coeficiente de difusión aumenta y esto equivale a mayor movimiento. Además de las conclusiones cualitativas, hizo predicciones cuantitativas que posteriormente pudieron ser comparadas con resultados experimentales.

Fueron Perrin y Chaudesaigues quienes entre los años 1908 y 1911 realizaron el experimento que comprobó la teoría de Einstein. ${ }^{24}$ El experimento consistió en tomar una serie de fotografías, registrando las posiciones de la partícula en intervalos de 30 segundos, al trazar los segmentos de líneas que unen estas posiciones registradas obtuvieron las trayectorias que se observan en la figura 1.2 z.

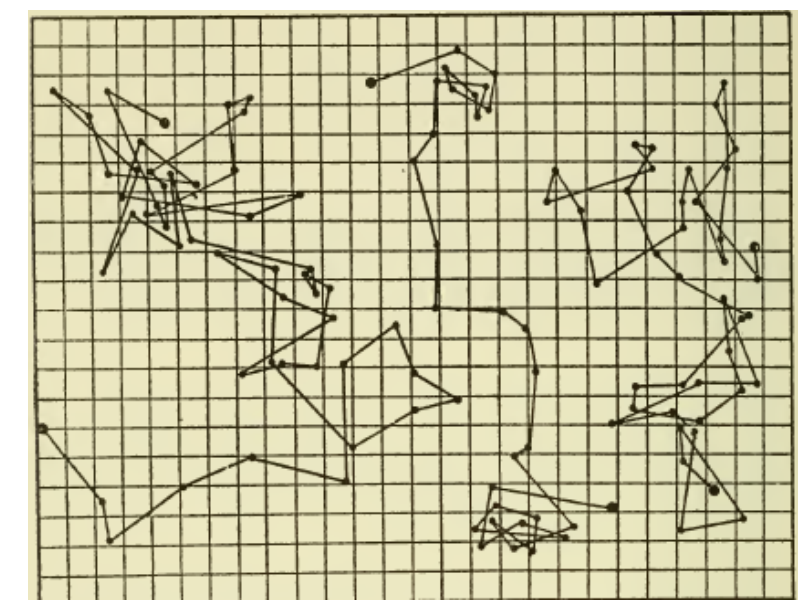

Figura 1.2: Se muestran tres dibujos registrados en el artículo de Perrin24 obtenidos al trazar los segmentos que unen las posiciones consecutivas del mismo grano en intervalos de 30 segundos.

Midiendo la distancia entre los puntos registrados en ese intervalo de tiempo, se obtuvieron los valores cuadráticos medios del desplazamiento, tal como se muestra en la imagen 1.3 .

Al graficar el desplazamiento cuadrático medio, observaron que, a partir de un cierto instante el valor del desplazamiento tenía un comportamiento lineal generando una recta como había predicho Einstein. La inclinación de esta recta es el coeficiente de difusión y pudo ser medido exitosamente. 


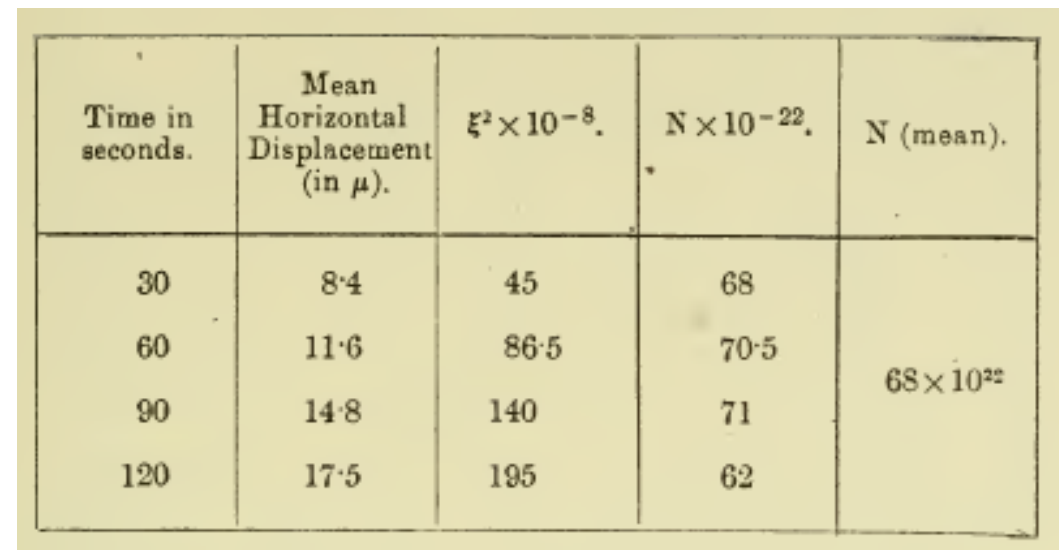

Figura 1.3: Tabla con una de las muchas medidas realizadas por Chaudesaigues. En este caso se realizó para 50 granos de radio $0.212 \mu \mathrm{m}$ inmersos en un fluido con una viscosidad de $0.012 \mathrm{~Pa} \cdot \mathrm{s}$ a una temperatura $\mathrm{T}=17^{\circ} \mathrm{C}$. Las medidas se tomaron cada 30 segundos. En la tabla se muestra el desplazamiento promedio horizontal, $\xi$ es el coeficiente de difusión dado por Einstein y el valor del número de Avogadro que obtuvieron 24

Puesto que experimentalmente ellos determinaban la temperatura, la viscosidad del fluido y las dimensiones de la partícula, a partir de la teoría de Einstein y con los datos obtenidos en el experimento lograron deducir el valor del número de Avogadro, el valor que obtuvieron fue de $6.4 \times 10^{23}$, su resultado fue muy bueno a pesar de todas las aproximaciones hechas, ya que actualmente el valor aceptado con mediciones más precisas del número de Avogadro es: $6.02 \times 10^{23}$. Perrin recibió el premio Nobel de Física en 1926 sus trabajos relativos a la discontinuidad de la materia y por el descubrimiento del equilibrio de sedimentación.

La maravillosa dupla que hicieron Einstein con su argumento y Perrin y Chaudesaigues con su comprobación experimental confirmaron la estructura atómica de la materia y esto impulsó el interés para estudiar el movimiento tan peculiar de esta partícula.

Posteriormente, Smoluchouski ${ }^{43}$ desarrolló un enfoque diferente del mismo problema con un método más directo que el usado por Einstein, él obtuvo una expresión del desplazamiento libre medio parecida a la de Einstein con una diferencia de un coeficiente de $64 / 27 \underline{\underline{19}}$

Dos años después del artículo de Einstein, en 1908, un físico francés llamado Langevin, inició un arduo estudio de procesos aleatorios que culminó en 1930 dando como resultado una descripción muy diferente pero igual de exitosa del movimiento Browniano en el que el movimiento es resultado de la suma de diferentes fuerzas, una fuerza de fricción debido al medio en dónde se encuentra la partícula y una fuerza fluctuante que genera su el movimiento aleatorio, esta fuerza tiene una distribución Gaussiana y la intensidad está determinada por el valor $q, q$ se puede relacionar con la temperatura del medio en el que se encuentra la partícula mediante el teorema de fluctuación-disipación, que nos dice que una vez alcanzado el estadio estacionario y el equilibrio térmico con el baño, las fuerzas fluctuantes se balancean con las fuerzas de fricción. 
Los sistemas pequeños desde ese entonces han sido ampliamente estudiados, gracias a todos los progresos tecnológicos el estudio de estos sistemas se ha facilitado y profundizado con aparatos que permiten tomar medidas y manipularlos. Todo este progreso nos ha encaminado estudiar lo que conocemos macroscópicamente traducido a sistemas micrométricos que presentan un movimiento Browniano. Tal es el caso de esta tesis con el estudio de máquinas térmicas.

Las máquinas térmicas generan trabajo mecánico a partir de la absorción de energía, muchos tipos de máquinas se han ideado desde tiempos muy remotos, el primer vestigio encontrado de una máquina térmica es la llamada "aeolipila", una turbina de vapor primitiva ${ }^{14}$ Fue hasta el periodo de la revolución industrial que el desarrollo de las máquinas térmicas tuvo un ímpetu, se comenzaron a usar para facilitar el trabajo de los obreros en las minas, se crearon fábricas, locomotoras, etc. A partir de ese momento surgieron grandes transformaciones tecnológicas, económicas, sociales y culturales. Este desarrollo estuvo aunado con la investigación de los fenómenos físicos que permitían la generación de trabajo a partir de energía; de estos estudios surgió la termodinámica. Cuando se desarrolló la teoría se encontró que los procesos de intercambio de energía estaban limitados por lo que llamaron las Leyes de la Termodinámica, el postulado de Clausius y de Carnot le adjudica al flujo de energía, sentido y limitaciones. Sabemos entonces que es imposible, o no se ha demostrado hasta ahora, la creación de una máquina térmica perpetua que transforme toda la energía absorbida en trabajo mecánico. La eficiencia de una máquina que trabaja en contacto con dos baños términos transforma energía en trabajo de manera limitada por la eficiencia del ciclo de Carnot, los avances tecnológicos buscan el desarrollo de máquinas cuya eficiencia sea lo más parecida a la del ciclo de Carnot. Sin embargo, el estudio de la termodinámica se hizo bajo supuestos muy ideales en la que los sistemas macroscópicos se encuentran siempre en equilibrio y no se toma en cuenta el tiempo del proceso ni sus pérdidas, aún así funcionó muy bien para el desarrollo de máquinas térmicas más eficientes. Sin embargo, posteriormente surgió el interés de investigar estos sistemas (aún macroscópicos) pero ahora fuera del equilibrio, Curzon y Ahlborn ${ }^{9}$ determinaron la eficiencia de un ciclo de Carnot para un tiempo finito, se demostró que la eficiencia tiene el valor $\eta=1-\sqrt{\frac{T_{l}}{T_{h}}}$. En donde $T_{h}$ es la temperatura de un baño caliente y $T_{l}$ es la temperatura de un baño frío por lo que se cumple que $T_{h}>T_{l}$.

La pregunta es ¿cómo proceder si ahora nuestro sistema es del tamaño de micras y tiene un movimiento aleatorio? Gracias a las técnicas de micro manipulación se han logrado realizar diferentes tipos de motores y máquinas micrométricas, por ejemplo: un nanomotor bio-hibrido de ADN que se desplaza por pistas predefinidas ${ }^{41}$ o elevadores moleculares. ${ }^{3}$ Incluso en 2016 se les otrogó el premio Nobel de química a Jean-Pierre Sauvage, Sir J. Fraser Stoddart y Bernard L. Feringa, quienes desarrollaron moléculas con movimientos controlables que podían realizar tareas cuando se les suministraba energía. ${ }^{28}$ También se ha logrado realizar experimentalmente ciclos de Stirling ${ }^{\sqrt{5}}$ y ciclos de Carnot ${ }^{20}$ con una partícula Browniana.

Aunque ha sido ampliamente estudiado el movimiento Browniano no queda del todo claro cómo describir la termodinámica traducida a sistemas pequeños. En la literatura se encuentran al menos dos líneas de estudio, la primera de ellas 
surgió con los trabajos de T. Hill, $\frac{15}{15}$ quien trató de desarrollar una termodinámica apropiada para los sistemas pequeños, sin embargo, hasta donde se ha podido ver, no ha tenido aplicaciones prácticas. Por otra parte la termodinámica estocástica desarrollada por Sekimoto ${ }^{34}$ nos permite calcular el trabajo, calor y entropía para una sola trayectoria de una partícula con movimiento aleatorio impulsado por las fluctuaciones del medio.

En la literatura existen varias investigaciones que se basan en los trabajos de Sekimoto y se dedican a hacer un análisis de las variables termodinámicas para diferentes procesos. Bao-Quan et al ${ }^{1}{ }^{1}$ hacen un análisis de micro motores y refrigeradores considerando que la partícula Browniana se mueve en un potencial tipo dientes de sierra y toman en cuenta que el ciclo sucede entre dos baños térmicos con los que el sistema está en equilibrio en su respectivo momento, encuentra que este ciclo es reversible cuando la máquina trabaja cuasi estáticamente y que la eficiencia nunca se aproxima a la del ciclo de Carnot. Schmield ${ }^{29}$ estudia un tipo de motor cuyos grados de libertad están sujetos a un potencial dependiente del tiempo acoplado de manera temporal a dos baños térmicos; en este caso, aunque el sistema tiene una dependencia temporal con los baños térmicos utilizan la relación de fluctuación-disipación y encuentra una expresión de la eficiencia diferente a la de Curzon-Ahlborn. Tu ${ }^{40}$ utilizando el formalismo de Sekimoto y la ecuación de Kramers desarrolla la teoría de una máquina térmica estocástica modificada por un potencial armónico dependiente del tiempo. En este caso, el autor utiliza estados de semi equilibrio y el teorema de fluctuación disipación en los que para cada tiempo la partícula Browniana se encuentra en equilibrio con el medio y utiliza lo que llaman "atajos a la adiabaticidad", en su artículo, encuentra que la eficiencia del ciclo a su máxima potencia es igual a la expresión de la eficiencia a la que llega Curzon y Ahlborn. Rana ${ }^{25}$ estudia el ciclo de Carnot de una partícula Browniana que es manipulada por un potencial armónico dependiente del tiempo, muestra que existen diferencias cualitativas entre el caso inercial y el caso sobre-amortiguado. Todas las cantidades termodinámicas, incluida la eficiencia, exhiben fuertes fluctuaciones incluso en el régimen cuasi estático y esto lo refuerza con una simulación. En todos estos trabajos se ha encontrado el cumplimiento de las leyes de la termodinámica partiendo del análisis de Sekimoto y tomando un promedio sobre muchas realizaciones del mismo proceso. A pesar del gran trabajo que han hecho, en todos ellos se ha aplicado el teorema de fluctuación-disipación el cual para sistemas que se encuentran fuera de equilibrio no está garantizada su validez, por lo que todos ellos suponen en sus cálculos un estado de equilibrio o semi equilibrio.

Esta tesis está inspirada en los trabajos mencionados, planteamos el estudio de una máquina térmica que trabaja en un ciclo de Stirling durante un tiempo finito $\tau$ en el caso sobre-amortiguado. En este modelo el sistema es sacado del equilibrio debido a un cambio generado por una fuerza externa y un cambio en la intensidad del ruido, es necesario tomar en cuenta que el sistema no se encuentra en equilibrio ni en semi equilibrio con el medio. Usamos el enfoque de Sekimoto para encontrar las variables termodinámicas de los procesos.

Se eligió el ciclo de Stirling ya que en el caso clásico su eficiencia puede llegar a ser la de un ciclo de Carnot, y todos sus procesos se pueden realizar mediante protocolos sencillos, de esta manera no nos metemos al problema de los procesos 
adiabáticos que solo se han resuelto mediante aproximaciones. A partir de esta tesis podremos cuantificar la influencia de las fluctuaciones en la eficiencia del ciclo y se llega a una solución general sin aproximaciones en la que se puede encontrar el valor de las variables termodinámicas dado un tiempo $\tau$ del proceso, las soluciones de esta tesis nos permiten extender lo aprendido a otro tipo de ciclos y tener una buena base de comparación.

Durante el desarrollo de la tesis comenzaremos con una descripción del método de micro manipulación más usado, las pinzas ópticas. Veremos la manera en la que los sistemas micrométricos pueden ser modificados de forma externa. En el capítulo 3 se presenta la definición de la termodinámica estocástica, de esta manera podremos relacionar la física de una partícula Browniana con la termodinámica del sistema, encontraremos las expresiones equivalentes para la primera ley de la termodinámica y para la entropía según el enfoque de Sekimoto.

Ya que el sistema se ve afectado por la manipulación de las pinzas ópticas, desarrollaremos la ecuación de Langevin y Fokker-Planck y tendremos una idea de cómo se ve afectado el movimiento de la partícula Browniana, primero en el caso más simple en el que no existe dependencia temporal y posteriormente el caso general en el que existe dependencia temporal generada por la manipulación de las pinzas ópticas.

Posteriormente describiremos cómo es que se puede realizar un ciclo de Stirling con una sola partícula Browniana y realizaremos un estudio de éste para el caso en cuasi equilibrio, en este caso no es necesario usar la termodinámica estocástica de Sekimoto. Para el caso del ciclo fuera de equilibrio en el Capítulo 6 primero proponemos un protocolo con el que es modificada la partícula Browniana y realizaremos el análisis completo de este caso que es totalmente dependiente del tiempo. Encontramos la ecuación de Langevin y de Fokker-Planck válidos para cada uno de los procesos. En el capítulo 7 se presentan los resultados del análisis termodinámico del ciclo cuando se encuentra fuera de equilibrio y en el capítulo 8 se tienen las conclusiones y persperctivas del trabajo. 


\section{Capítulo 2}

\section{Relación Experimental}

El progreso de la tecnología no siempre se ha desarrollado de la mano del progreso científico. Un ejemplo claro de esto es la termodinámica, ya que la primera máquina térmica fue ideada en el año 130 a.C. mientras que los conceptos que nos ayudan a entenderlas de manera termodinámica comenzaron a surgir a partir del año 1803 con la definición del calor específico y calor latente. ${ }^{14}$ Alrededor de 200 años después, el desarrollo de la tecnología ha sido increíblemente amplio, abarca desde exploraciones espaciales no tripuladas hasta la manipulación de estructuras moleculares. Todo el progreso tecnológico ha impulsado también un amplio desarrollo de la ciencia.

Con la micro-nano tecnología ahora somos capaces de controlar partículas muy pequeñas a nuestro gusto y esto abre puertas a una gran cantidad de experimentos en el mundo micrométrico. En este capítulo veremos cómo es que el desarrollo de las pinzas ópticas contribuye al estudio de sistemas micrométricos.

\subsection{Pinzas ópticas}

Las pinzas ópticas son un mecanismo de captura y manipulación óptica que consiste de un haz de luz altamente enfocado capaz de sostener partículas micrométricas. Se ha convertido en una herramienta muy versátil y utilizada para la micromanipulación ya que un sistema de pinzas ópticas es capaz de clasificar, direccionar y transportar cualquier objeto nano o micrométrico, incluso objetos biológicos como glóbulos rojos pueden ser manipulados sin alterar su composición. $\frac{37}{37}$

Arthur Ashkin fue quien desarrolló la idea básica para atrapar objetos usando la fuerza de radiación de la luz láser. Sin embargo, la idea de que la luz puede ejercer una presión surgió hace varios siglos atrás. En 1873 Kepler postuló que la presión de la luz es la razón por la cual las colas de los cometas siempre van en dirección contraria al sol.

Posteriormente Maxwell demostró con su teoría del electromagnetismo que la luz podía ejercer una fuerza y además encontró una equivalencia entre la presión de radiación con la densidad de energía que transporta una onda electromagnética. La existencia de la presión de radiación pudo comprobarse cuando se desarrolla- 
ron sistemas de alta densidad de energía a principios del año 1900. La invención de láser impulsó las investigaciones sobre la presión de radiación y fue en 1970 que Ashkin realizó las primeras investigaciones que llevarían a las pinzas ópticas.

Al hacer un análisis de las fuerzas que se ejercen sobre la partícula debido al láser podremos comprender el fenómeno. ${ }^{22}$ La presión de radiación empuja a la partícula con una fuerza $F_{\text {scat }}$, ésta es la fuerza de dispersión que se muestra en la figura 2.1. Además de esta fuerza existen otras debidas al cambio del medio por el que pasa el haz. Imaginemos un rayo " $a$ ", primero se propaga por un medio con un índice de refracción $n_{m}$ en el momento en el que el haz de luz incide sobre una partícula, cambia el medio de propagación, se genera un cambio en el índice de refracción $n$, el cual es más grande que el del medio que rodea a la partícula $n_{m}$, esto genera un cambio en el momento del haz y debido a la conservación del momento la partícula también experimenta un cambio de momento de la misma magnitud pero en dirección opuesta que da lugar a la fuerza $F_{a}$. Esto pasa de la misma manera con otros rayos formados por el haz, por ejemplo un segundo rayo "b"generando la fuerza $F_{b}$.

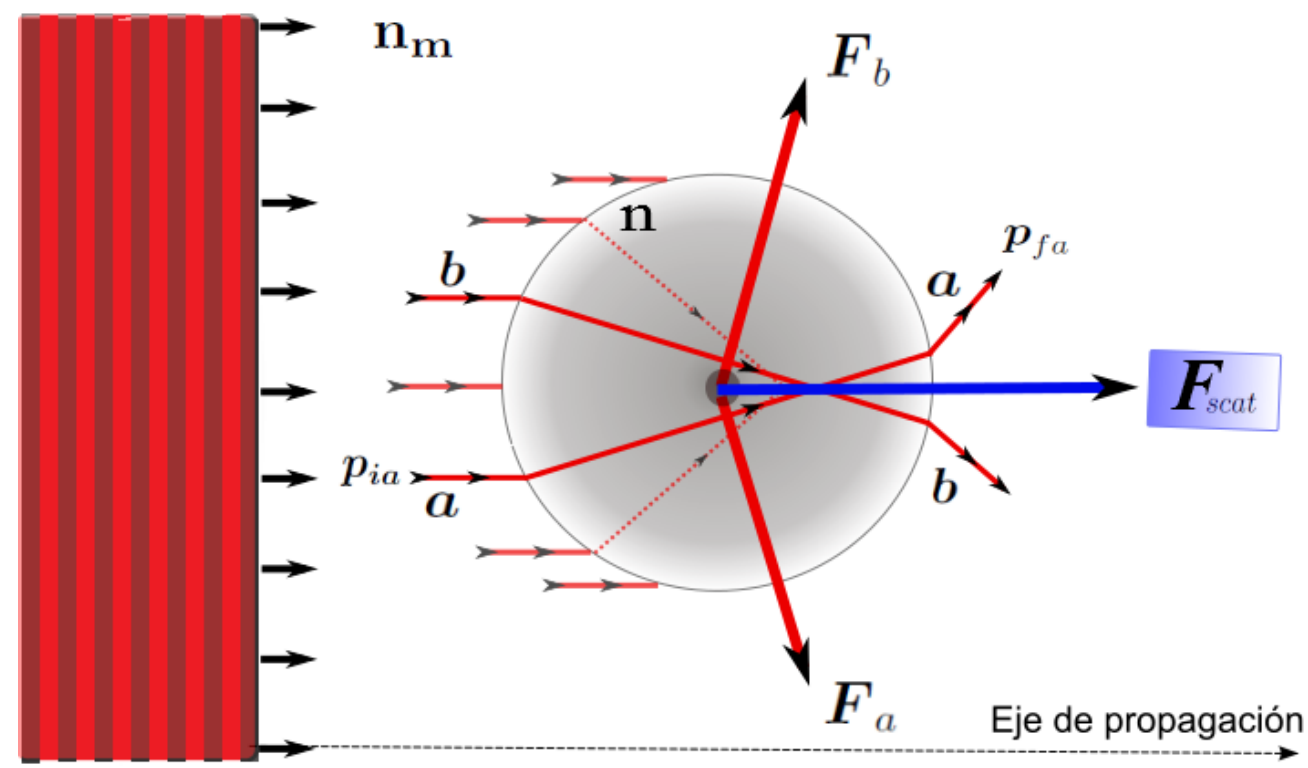

Figura 2.1: De la referencia (22) se muestra el diagrama ilustrativo de las fuerzas que actúan sobre la partícula generadas por un haz plano, cuando cambia el medio por que el pasa el haz, la fuerza $F_{a}$ es generada por el rayo a y $F_{b}$ es generado por el rayo b y la fuerza de dispersión $F_{\text {scat }}$ es la fuerza generada por la presión de radiación.

Vemos entonces que debido al haz de luz que actúa sobre la partícula, actúan la fuerza de dispersión $F_{\text {scat }}$ y las fuerzas debidas al cambio en el medio.

Cuando se trabaja con un haz que tiene perfil Gaussiano, el cambio de momento provocado al encontrar un medio diferente genera una fuerza cuyos componentes transversales dan origen a una fuerza de gradiente $\left(F_{\text {grad }}\right)$ que empuja a la partícula hacia la región donde el gradiente es mayor, que coincide con la dirección transversal al eje de propagación, como se puede ver en la figura 2.2. Es decir, la partícula es atraída hacia el máximo del haz Gaussiano 


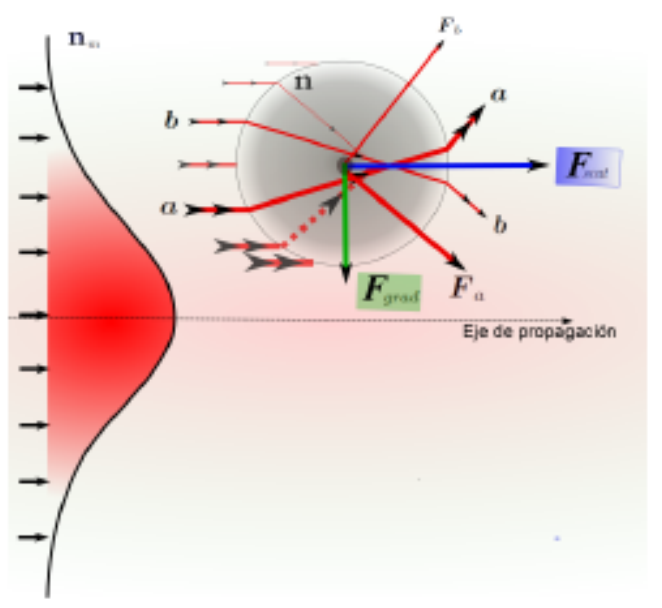

Figura 2.2: Diagrama de las direcciones de las fuerzas que son generadas con un haz Gaussiano.22

Únicamente con la fuerza de gradiente y la fuerza de dispersión fueron ideadas las primeras pinzas ópticas que consistían en el atrapamiento de partículas utilizando dos haces de luz con la misma intensidad, como la que se muestra en la figura2.3.

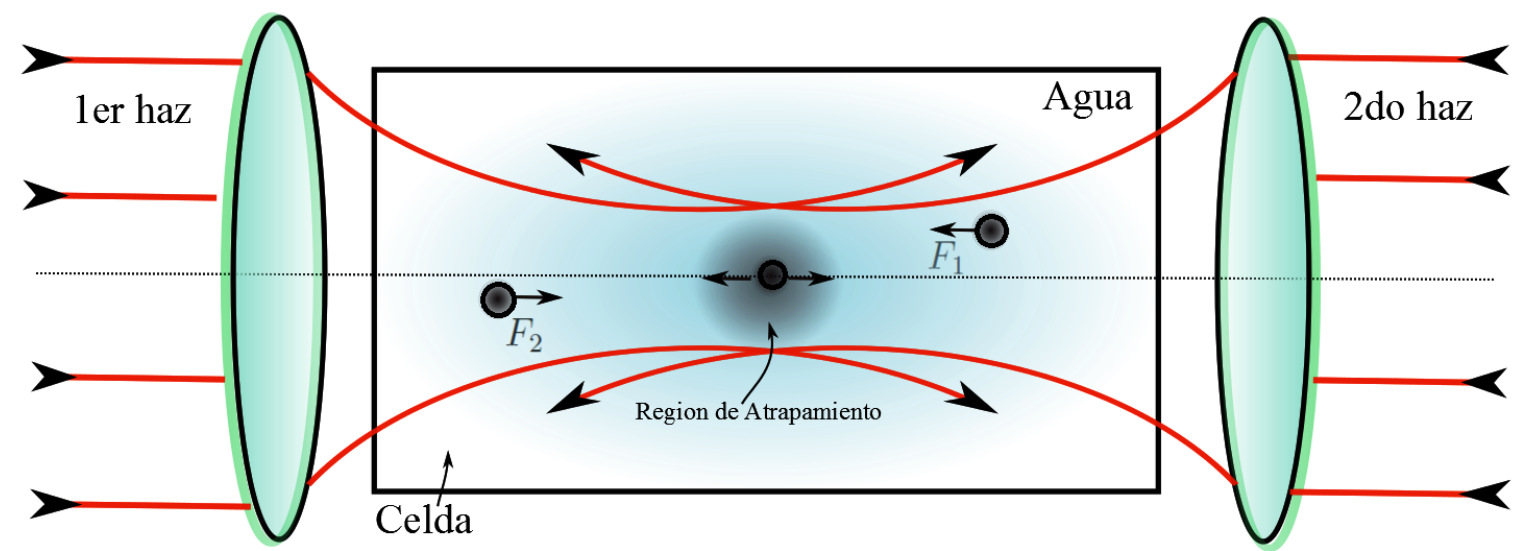

Figura 2.3: Modelo de la primera primera trampa óptica realizada con dos haces de luz Gaussianos, la fuerza gradiente va en dirección del máximo del haz.22

\subsubsection{Pinzas ópticas de un solo haz}

Para generar una trampa óptica de un solo haz con perfil Gaussiano, es necesario enfocar el haz láser a través de un objetivo de microscopio con alta apertura numérica, al hacer esto el haz tiene una rápida convergencia a un punto focal, este arreglo es el más común de pinzas ópticas. Para entender mejor cómo es que la convergencia genera el atrapamiento descomponemos el haz total en rayos individuales que se propagan en forma paralela y en línea recta, en la figura 2.4 podemos ver la trayectoria que siguen dos rayos incidiendo sobre la superficie de una esfera.

El efecto que tiene la fuerza gradiente generada por el perfil Gaussiano del láser y 


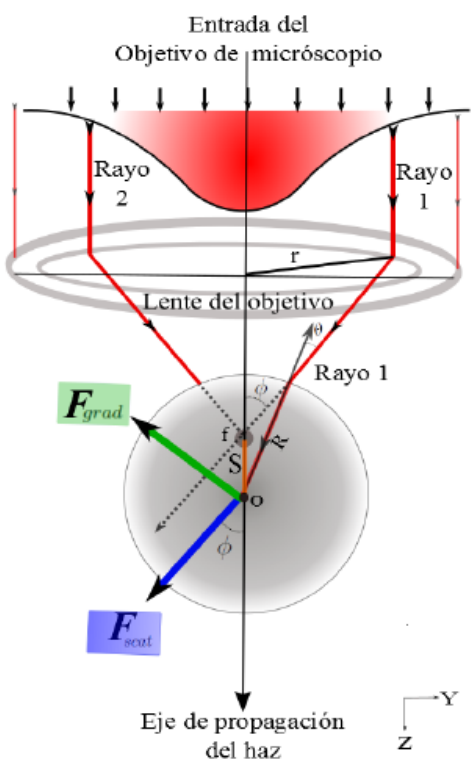

Figura 2.4: Se muestra el efecto del la fuerza de dispersión y gradiente sobre una esfera, las cuales generan el atrapamiento cuando se está usando un lente objetivo.22

la fuerza de esparcimiento generada por la presión de radiación al estar enfocado por el objetivo, es un cambio de dirección de las fuerzas, ahora la fuerza de dispersión empuja a la esfera en una dirección que no coincide con la dirección de propagación sobre el eje $z$ y de la misma manera sucede con la fuerza gradiente, pero además de eso la componente en $z$ de la fuerza de dispersión va en dirección contraria a la de propagación.

El balance entre la componente en la dirección $z$ de ambas fuerzas determina que ocurra o no el atrapamiento en las pinzas ópticas. En la figura 2.5 notamos que si la componente $z$ de la fuerza de gradiente es dominante entonces ocurre el atrapamiento, de otro modo la fuerza de esparcimiento empuja a la esfera en dirección del haz incidente más allá del punto focal, en este caso el signo de la fuerza de gradiente depende de la distancia $S$ que va del centro de la esfera, al punto focal $f$. Si el centro de la esfera está adelante o atrás del punto focal del objetivo, la fuerza de gradiente atraerá o empujará a la esfera hacia ese punto, generándose en esa región una trampa estable tridimensional.

La partícula confinada en el campo óptico actúa en una primera aproximación para desplazamientos pequeños como un oscilador armónico ${ }^{39}$, como se puede ver en la Figura 2.6. Este hecho se investigó teóricamente durante el desarrollo de las pinzas ópticas en $1982 !^{17} \mathrm{Y}$ aunque se ha demostrado que la aproximación no es correcta cuando se toman en cuenta desplazamientos grandes ${ }^{26}$ para fines de esta tesis se puede utilizar la aproximación de un oscilador armónico.

Las pinzas ópticas se han convertido en una herramienta de bastante utilidad en el estudio de micro-sistemas y con mucho potencial en varios campos.

Un ejemplo de las aplicaciones de las pinzas ópticas es el control de nanopartículas para generar ciclos mediante la manipulación de parámetros externos. Se han 

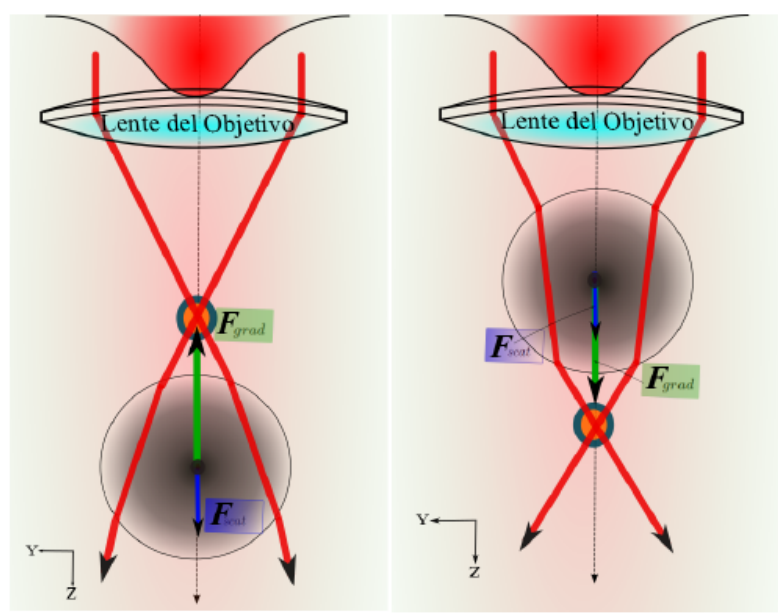

Figura 2.5: Diagrama de las fuerzas sobre la partícula cuando su centro se encuentra adelante o atrás del punto focal.22

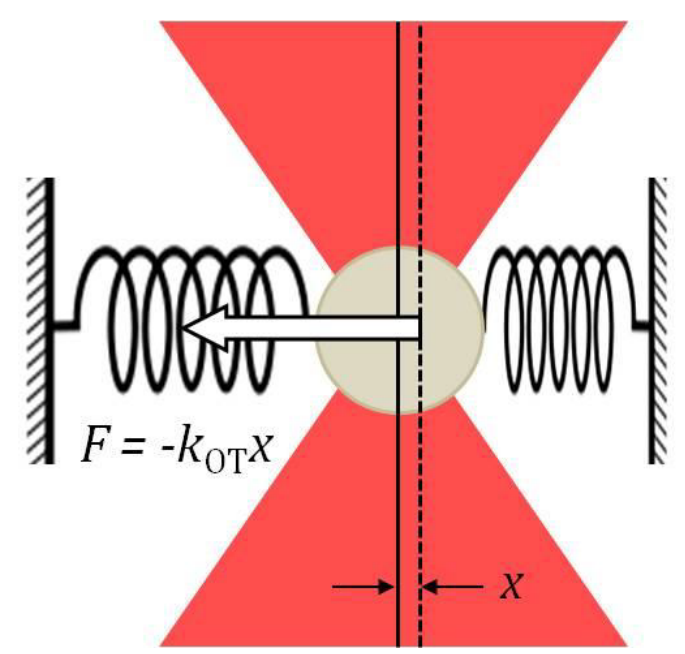

Figura 2.6: Potencial generado por las pinzas ópticas visto como un resorte de Hooke lineal. 39

realizado varios estudios sobre el tema, el más influyente para la realización de esta tesis es el siguiente modelo experimental realizado por I. Martínez et al. en su artíclulo llamado Adiabatic Processes Realized With a Trapped Brownian Particle ${ }^{21}$ por lo que explicaremos detalladamente el proceso.

\subsection{Modelo experimental}

Martínez y su equipo idearon un modelo experimental de pinzas ópticas para atrapar a una partícula Browniana y manipularla. La manera en la que efectúan su modelo experimental les permite variar parámetros externos para realizar todo tipo de procesos termodinámicos con la partícula atrapada.

Como explicamos anteriormente, para obtener las pinzas ópticas se necesita de un láser, en este caso de $980 \mathrm{~nm}$ altamente enfocado por un objetivo $O_{1}$ con apertura numérica grande, como se ve en la figura 2.7. Esto genera el potencial óptico tipo oscilador armónico necesario para atrapar a la partícula Browniana. 
Las partículas son esferas de poliestireno con $1.00 \pm 0.05 \mu$ de diámetro diluidas en agua de manera que la concentración de esferas por milímetro es muy baja y se encuentran en una cámara sellada para evitar flujos y contaminación. Esto permite un óptimo estudio de la partícula Browniana confinada por las pinzas ópticas.

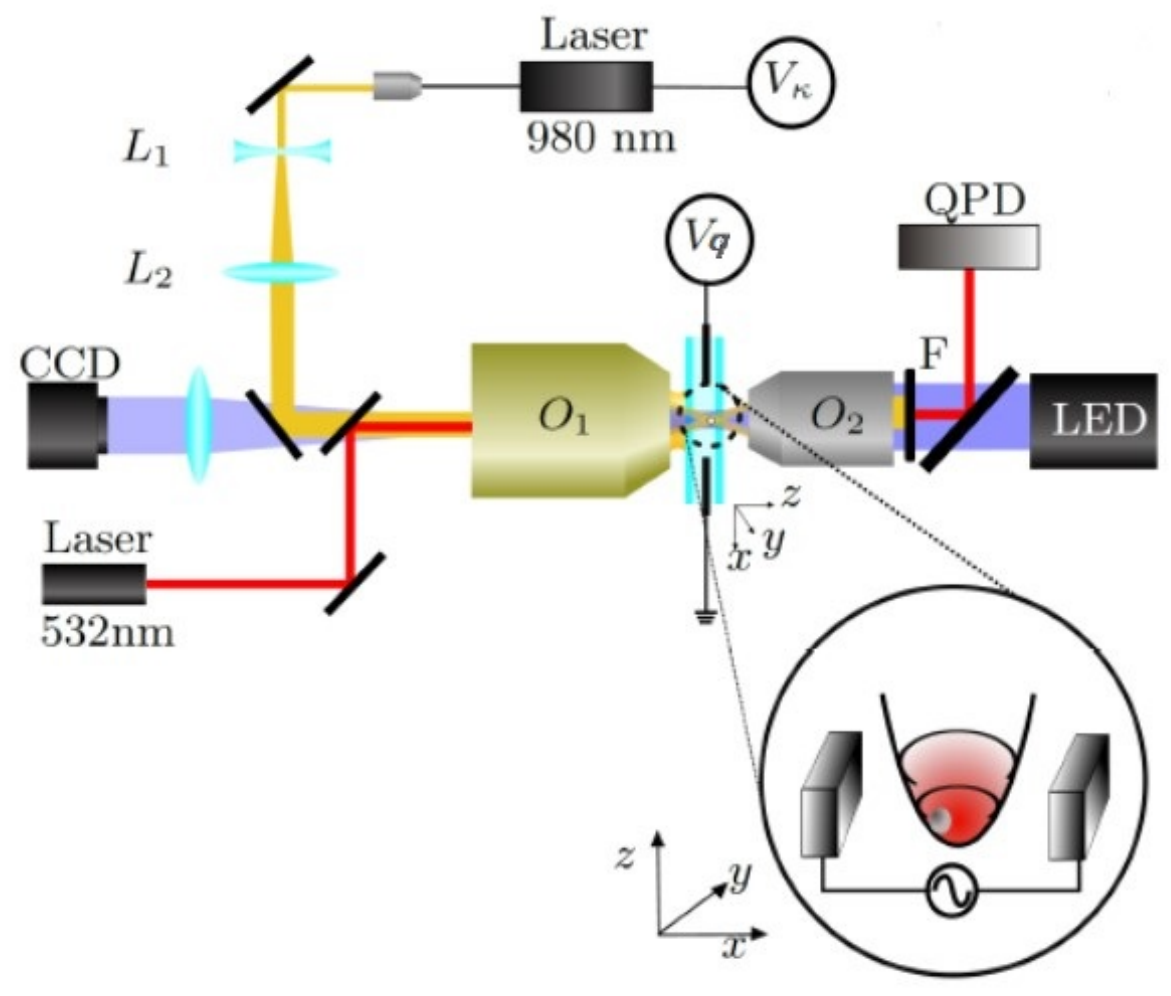

Figura 2.7: Arreglo experimental de pinzas ópticas con dos potenciales externos, $V_{k}$ conectado al láser y $V_{q}$ conectado a la cámara donde se encuentra la partícula!21

En el modelo para manipular a la partícula Browniana se aplican dos voltajes en diferentes secciones del experimento, de tal manera que la manipulación externa de estos voltajes genera que la partícula Browniana atrapada pase por diferentes procesos. Un voltaje que puede ser manipulado externamente, $V_{k}$, es aplicado directamente al láser, lo que sucede al modificar el voltaje externo es que cambia la potencia óptica del láser. La potencia está ligada de manera lineal con la rigidez de la trampa óptica, por lo tanto, al modificar el voltaje externo $V_{k}$, la rigidez de la trampa es controlada en la misma proporción. En el caso en el que modelamos el potencial de las pinzas ópticas como un potencial tipo oscilador armónico en una dimensión el cambio en el voltaje externo es directamente proporcional al cambio de la constante elástica o constante de rigidez del potencial.

El segundo voltaje externo controlable $V_{q}$ es aplicado a la cámara sellada en la que se encuentran las esferas mediante dos electrodos de aluminio que son colocados en los extremos como se muestra en la figura 2.7. El voltaje resultante controla la intensidad del ruido que siente la partícula Browniana que en una aproximación puede ligarse a la temperatura efectiva de la partícula. 
Con estos dos voltajes podemos controlar a voluntad, de manera externa e independiente, la rigidez de la trampa y la intensidad del ruido que siente la partícula. El control total de estos parámetros nos permite formular una gran variedad de procesos termodinámicos.

En el cuadro 2.1 podremos encontrar los datos utilizados por Martínez para la realización del experimento.

\begin{tabular}{||c|c||}
\hline \multicolumn{2}{||c||}{ Datos experimentales } \\
\hline \hline Láser & $\begin{array}{c}\text { Longitud de onda } \lambda=980 \mathrm{~nm} \\
\text { Potencia Máxima } 100 \mathrm{~mW}\end{array}$ \\
\hline Objetivo & CFI PL FL $100 \times N A 1.30$ apertura numérica 1.30 \\
\hline$V_{k}$ & Potencia máxima $250 \mathrm{kHz}$ \\
\hline Esferas & Material: Poliestireno \\
& Diámetro: $(1.00 \pm 0.05) \mu \mathrm{m}$ \\
\hline Medio: Agua & Viscosidad $\eta=1.0 \times 10^{-3} \mathrm{Kg} /(\mathrm{ms}) \mathrm{a} 293.15 \mathrm{~K}$ \\
\hline Proceso isotérmico & con $T=300 \mathrm{~K}$ y $k$ pasa de: $k_{0}=(5.0 \pm 0.2) p N / \mu \mathrm{m}$ \\
& $\mathrm{a} k=(28.0 \pm 0.2) \mathrm{pN} / \mu \mathrm{m}$ \\
\hline Proceso isócoro & con $k=(10.0 \pm 0.2) p N / \mu m \mathrm{y} \mathrm{T}$ pasa de: $T_{0}=300 \mathrm{~K}$ \\
& $T=1200 \mathrm{~K}$ \\
\hline Duración de los protocolos & $\tau=0.5 \mathrm{~s}$ \\
\hline
\end{tabular}

Cuadro 2.1: Tabla con los valores numéricos del experimento realizado por Martínez.21

Tomando en cuenta el control de ambos parámetros externos gracias a las pinzas ópticas podemos proponer un protocolo para generar un ciclo de Stirling con una partícula Browniana. Actualmente, muchas de las más poderosas técnicas de micro-manipulación son derivadas de las pinzas ópticas; se ha logrado llevar acabo reacciones fotoquímicas, tales como la polimerización por absorción multifotónica y es conveniente para la fabricación de objetos biológicos y químicos en tiempo real. Por todo el gran impulso de las pinzas ópticas al estudio de los sistemas pequeños, Arthur Ashkin fue premiado con el Nobel de física del 2018.

Ya que conocemos el funcionamiento de las pinzas ópticas y la manera en la que nos pueden ayudar a estudiar sistemas micrométricos y en este caso a realizar procesos termodinámicos, resulta necesario determinar qué es la termodinámica en sistemas micrométricos mejor conocida como termodinámica estocástica. 



\section{Capítulo 3}

\section{Termodinámica estocástica}

La termodinámica clásica es una ciencia fenomenológica, es decir se encuentra basada en postulados generales inferidos de innumerables estudios experimentales, nos indican la manera en la que se realiza el proceso de intercambio de energía en sistemas macroscópicos que se encuentran en equilibrio. A partir de estos postulados es posible determinar las características macroscópicas de una sustancia dada durante un proceso, como el calor, el trabajo y la entropía.13 Por otro lado, con la mecánica clásica o hamiltoniana es posible determinar la evolución temporal del movimiento de una partícula clásica. Conociendo la evolución determinista de las moléculas que componen a un fluido, podemos hacer uso de la mecánica estadística para determinar la termodinámica de equilibrio del sistema. Sin embargo, existe un nivel intermedio entre la dinámica hamiltoniana microscópica y la termodinámica macroscópica. Este nivel define la dinámica de moléculas de tamaño mesoscópico que son del orden de micras, la dinámica de las partículas en este nivel además de encontrarse bajo la influencia de fuerzas de índole diversa, también sienten la influencia de las colisiones con las moléculas del medio en el que están inmersas. Para sistemas de este tamaño tendremos una dinámica estocástica donde las fluctuaciones no pueden despreciarse. Existen varias formas de estudiar este movimiento. Pero ¿cómo es la termodinámica para sistemas de este tamaño?, ¿cómo se puede encontrar a partir de la dinámica de estos sistemas estocásticos una relación con la termodinámica en equilibrio y fuera de equilibrio que conocemos?, ¿la termodinámica de estos sistemas tendrá propiedades termodinámicas que no conocemos para sistemas mesoscópicos? Estas son unas de las muchas cuestiones con las que trata la termodinámica estocástica.

Existen dos enfoques principales para el estudio de la termodinámica de sistemas pequeños. La desarrollada por Hill ${ }^{15}$, quien hace una generalización de las ecuaciones de la termodinámica en las que se debe de incluir una variación en el tamaño del sistema. Por ejemplo $N$ el número de grados de polimerización o agregación y $V$ el volumen del sistema. En su trabajo, Hill utiliza dos conceptos de mecánica estadística: el concepto de ensambles lo lleva a una escala de sistemas pequeños, y el postulado fundamental de la mecánica estadística el cual indica que el promedio temporal para un solo sistema es igual al promedio del ensamble correspondiente. 
Por otro lado, la termodinámica estocástica combina lo que Sekimoto llamó energética estocástica, ${ }^{33}$ con la idea de que la entropía puede ser aplicada consistentemente a una sola trayectoria fluctuante. Sekimoto hizo un estudio de la termodinámica para sistemas pequeños que se basa en la ecuación de Langevin. 34

Langevin buscó la forma de aplicar la segunda ley de Newton a una partícula Browniana, escribiendo una versión de esta ley para procesos estocásticos. De esta manera se logró explicar la dinámica estocástica mediante la existencia de fuerzas y con ésta se puede determinar la trayectoria aleatoria de una partícula Browniana. La aparente simplicidad de este enfoque se logra con el coste de forzar la existencia de nuevos objetos matemáticos con propiedades extrañas: el cálculo estocástico.

En su artículo Langevin ${ }^{19}$ pudo determinar una aplicación correcta del método de Smoluchowski que lo llevó a recuperar la fórmula de Einstein. Su razonamiento fue el siguiente ${ }^{\sqrt[6]{6}}$ La fuerza total que experimenta la partícula en una dimensión cuando se encuentra en un fluido, es la suma de dos fuerzas: una fuerza que sucede en una escala de tiempo del orden de segundos, es la sistemática, y otra que genera en la partícula un movimiento irregular, la fuerza fluctuante.

$$
F=F_{s}(t)+F_{f}(t)
$$

$F_{s}(t)$ es la fuerza sistemática y está compuesta por la suma de fuerzas externas que actúan sobre la partícula; la fuerza de fricción que actúa sobre la partícula Browniana debido al medio en el que se encuentra y una fuerza debida a una influencia externa. De acuerdo a la hidrodinámica la fuerza de fricción se genera en sentido opuesto a la velocidad relativa y depende de ésta

$$
F_{\text {fric }}=-\alpha v \text {. }
$$

donde $\alpha$ es el coeficiente de fricción del medio y $v$ es la velocidad relativa de la partícula Browniana respecto del fluido en que está sumergida. En 1851 Stokes determinó que esta fuerza para objetos esféricos moviéndose en un flujo viscoso con número de Reynold pequeño es

$$
F_{\text {fric }}=-6 \pi r \eta v
$$

en donde $\eta$ es la viscosidad del fluido, $v$ la velocidad de la esfera y $r$ el radio de la esfera. La fuerza sistemática sería la suma de la fueza hidrodinámica y la fuerza externa.

$$
F_{s}=F_{\text {fric }}+F_{\text {ext }}
$$

La fuerza fluctuante generada debido al medio en el que se encuentra la partícula, no está correlacionada entre sí en distintos instantes de tiempo, esto significa que el valor de la fuerza estocástica en un instante dado no tiene relación con la fuerza en otro instante, debido a que en este intervalo de tiempo del orden de segundos la fuerza varía muchísimo; esta propiedad permite realizar la suma de las fuerzas. La podemos determinar como la masa por una variable aleatoria, $\Gamma(t)$, que es mejor conocida como ruido

$$
F_{f}=m \Gamma(t),
$$


es el ruido el que genera que la fuerza tenga propiedades aleatorias. Langevin propuso al ruido con una distribución Gaussiana, esto implica que asumimos que su promedio sobre el ensamble de muchas realizaciones es igual a cero

$$
\langle\Gamma(t)\rangle=0
$$

y su correlación temporal satisface que a diferentes tiempos no existe relación entre las $\Gamma$

$$
\left\langle\Gamma(t) \Gamma\left(t^{\prime}\right)\right\rangle=q \delta\left(t-t^{\prime}\right)
$$

Tomando esto en cuenta las fuerzas se pueden sumar y el resultado de la suma también va a ser aleatorio.

$$
F=-\alpha v(t)+F_{\text {ext }}+m \Gamma(t)
$$

Por otro lado, de acuerdo con los principios de la mecánica Newtoniana la fuerza que experimenta una partícula determina la trayectoria y la velocidad que tiene, esto implica que tanto la posición como la velocidad de la partícula son también cantidades fluctuantes y se tendrán que determinar sus distribuciones en términos de las propiedades de la distribución de $\Gamma(t)$.

La ecuación de Langevin para una partícula Browniana en donde $\gamma=\frac{\alpha}{m}$ está dada por 27

$$
\begin{aligned}
\dot{v} & =-\gamma v(t)+\frac{F_{\text {ext }}}{m}+\Gamma(t) \\
\dot{x} & =v
\end{aligned}
$$

Sekimoto se basa en la ecuación de Langevin para realizar una analogía con la termodinámica clásica, puesto que la ecuación de Langevin modela la dinámica de una partícula tomando en cuenta su interacción con un ambiente térmico de la misma manera en la que la termodinámica clásica explica la interacción entre sistema, ambiente y sistema externo. Esta analogía la realiza de la siguiente manera:

Un sistema clásico se define como una porción del universo delimitada, con al menos $10^{23}$ partículas. En el caso mesoscópico el sistema está representado por la posición y momento de la partícula Browniana descrita por la ecuación de Langevin.

El ambiente térmico o baño es el sistema de fondo con el cual se encuentra en contacto el sistema, en este caso la interacción se puede despreciar ya que la energía transferida es muy pequeña comparada con la energía del ambiente, normalmente está caracterizado por una temperatura. En el caso mesoscópico la interacción entre el sistema y el ambiente térmico está caracterizada por el coeficiente de fricción $\gamma$ y la intensidad de la fuerza aleatoria $\Gamma(t)$.

El sistema externo en termodinámica es un agente que es capaz de controlar macroscópicamente el sistema, entre ellos existe un intercambio de energía, por ejemplo mediante un cambio de volumen y a veces de masa. En el caso mesoscópico el sistema externo es un sistema cuya dinámica no está 
determinada por la ecuación de Langevin y es capaz de controlar y sacar del equilibrio al sistema.

El enfoque de Sekimoto ha sido ampliamente utilizado debido a que se puede hacer un análisis energético sobre una sola trayectoria de la partícula Browniana, encontrando el trabajo, calor y entropía. Ha sido utilizado en sistemas mesoscópicos como coloides o biomoléculas llevadas fuera de equilibrio por fuerzas que tienen una dependencia temporal. 32

\subsection{Primera ley de la termodinámica}

Como hemos visto la ecuación de Langevin es una ecuación de balance de fuerzas entre la interacción de la partícula, el medio y cualquier sistema externo. Siendo así, es posible derivar a partir de ella una ecuación de balance de energía, que debido al paralelismo con la termodinámica sería el equivalente a la primera ley de la termodinámica y nos indicará la relación entre el trabajo aplicado o extraído, el intercambio de calor y los cambios en la energía generada sobre una sola trayectoria fluctuante. Al derivarse de una ecuación estocástica la energía tendrá entonces un carácter aleatorio generado por el ruido térmico, el mismo causante del movimiento Browniano de la partícula de estudio. $\underline{31}$

Escribiendo la ecuación de Langevin para un sistema en una dimensión en el caso sobre amortiguado, en el que podemos despreciar el término en la ecuación que es debido a la inercia y además tomando en cuenta que está siendo modificada por la presencia de una fuerza externa generalizada derivable de un potencial $U(x, a)$ que depende de la posición y de un parámetro externo $a, F_{e x t}=-\frac{d U}{d x}$, en el caso sobre amortiguado la ecuación de Langevin es

$$
0=-\gamma \frac{d x}{d t}-\frac{d U(x, a)}{d x}+\Gamma(t)
$$

en donde $x$ es la variable dinámica del sistema y es aleatoria, $\gamma$ es la constante de fricción del medio por unidad masa y $\Gamma$ es conocido como el ruido térmico. El primer término $-\gamma \frac{d x}{d t}$ nos indica la fuerza sistemática que ejerce el medio sobre la partícula, el segundo es la fuerza debida al potencial externo y el último corresponde al de la fuerza fluctuante que también nos indica un tipo de interacción entre la partícula y el medio. $\underline{33}$

Debido al carácter aleatorio del sistema el cálculo efectuado puede realizarse en el sentido del cálculo de Ito ${ }^{16}$ o del cálculo de Stratonovich. ${ }^{[36}$ Las reglas de cálculo de Itô y de Stratonovich son diferentes, pero ambas son matemáticamente consistentes, el enfoque de Stratonovich es el que usaremos debido a la forma de la ecuación diferencial con la que tratamos, ya que es lineal y tiene ruido blanco, las reglas formales del cálculo tal como las integrales por partes, cambios de variables y la regla de la cadena se mantienen como las conocemos en este enfoque. $\frac{35}{3}$

Si suponemos que el estado del sistema ha cambiado un $-d x$ entonces la multiplicación de la fuerza dada por la ecuación de Langevin por este cambio representa un balance de energía. Al hacer la multiplicación con el cálculo de Stratonovich 
obtenemos la siguiente expresión de la energía

$$
\begin{aligned}
& 0=\gamma \frac{d x}{d t} \circ d x+\frac{d U(x, a)}{d x} \circ d x-\Gamma(t) \circ d x \\
& 0=-\left(-\gamma \frac{d x}{d t}+\Gamma\right) \circ d x+\frac{d U(x, a)}{d x} \circ d x
\end{aligned}
$$

el primer término del lado derecho de la ecuación (3.11) corresponde a la fuerza de reacción ejercida por el sistema sobre el baño térmico (que sea de esta manera y no la fuerza ejercida por el baño sobre el sistema se debe al signo en $-d x$ que hemos multiplicado y la convención que utilizaremos). El segundo término es la energía debida al cambio del potencial.

Aunque el movimiento microscópico de las partículas del medio no está representado explícitamente en la ecuación de Langevin, la ley de acción y reacción nos permite identificar la cantidad de energía que ha sido transferida. Esta cantidad varía en cada realización incluso en el signo pero se ha logrado extender exitosamente el concepto que ha sido utilizado en el estudio de máquinas térmicas principalmente en dos $\operatorname{casos}^{2}$

1 En máquinas térmicas que tienen un potencial periódico estático y una temperatura que cambia con el tiempo $8[18] 42$

2 En máquinas que tienen motores con un potencial armónico dependiente del tiempo y una temperatura temporalmente modulada.11] 12,29

\subsubsection{Calor}

La energía que es transmitida por medios no mecánicos es llamada calor, en el caso mesoscópico el calor surge de la energía que es transmitida entre el sistema y el medio, es una energía que se encuentra siempre presente debido a la fuerza restitutiva $-\gamma d x / d t$ y al ruido térmico $\Gamma(t)$ que juntas ejercen una fuerza $-\gamma d x / d t+\Gamma(t)$ sobre la partícula. Tomando en cuenta que se cumple la ley de acción y reacción entonces la partícula ejerce una fuerza de reacción $-(-\gamma d x / d t+\Gamma(t))$ sobre el medio.

Al tomar en cuenta que hay una evolución de la posición del sistema $x$ sobre un intervalo de tiempo $d t$ podemos determinar el trabajo hecho por el medio sobre la partícula en ese intervalo de tiempo. El intercambio de energía que es ejercido por el medio sobre la partícula lo definimos como calor transferido, el cual puede ser positivo o negativo.

$$
d^{\prime} Q=\left(-\gamma \frac{d x}{d t}+\Gamma(t)\right) \circ d x(t)
$$

Sustituyendo este valor en la ecuación de Langevin llegamos a una segunda expresión para el calor.

$$
0=-d^{\prime} Q+\frac{d U(x, a)}{d x} \circ d x
$$




$$
d^{\prime} Q=\frac{d U(x, a)}{d x} \circ d x
$$

La convención de signos que usaremos es la siguiente: cuando se tiene una cantidad positiva de trabajo se entiende que es el sistema el que está recibiendo calor del medio en el que se encuentra. En el caso contrario en el que el trabajo es negativo entonces el sistema está dando calor al medio.

Tomando en cuenta la definición de calor que acabamos de describir seguiremos con el desarrollo de la ecuación de balance de energía derivada de la ecuación de Langevin

$$
0=-d^{\prime} Q+\frac{d U}{d x} \circ d x
$$

Habíamos mencionado que la energía potencial no solo depende de la posición, también se ve influenciada por un parámetro externo $a(t)$, la dependencia en el potencial del parámetro externo representa un cambio en la forma del potencial. Cuando $U(x, a)$ representa un contenedor rígido el parámetro $a$ es el que controla la posición de la barrera. En el caso del potencial tipo oscilador armónico, el parámetro externo es el que controla la rigidez. Tomando en cuenta esto la diferencial de la energía potencial es

$$
d U(x(t), a(t))=\frac{d U(x(t), a(t))}{d x} \circ d x+\frac{d U(x(t), a(t))}{d a} \circ d a
$$

Realizando la sustitución correspondiente en la ecuación de balance

$$
0=-d^{\prime} Q+d U(x(t), a(t))-\frac{d U(x(t), a(t))}{d a} \circ d a
$$

Por otro lado la energía interna del sistema para el caso sobreamortiguado desprecia el término de la energía cinética y tiene la siguiente expresión

$$
E=U(x, a)
$$

La ecuación de balance de energía hasta el momento queda de la siguiente forma,

$$
d E=d Q+\frac{d U}{d a} \circ d a
$$

se puede observar una gran analogía con la primera ley de la termodinámica

\subsubsection{Trabajo}

Siguiendo el desarrollo de la ecuación de balance de energía podemos identificar el segundo término del lado derecho de la ecuación con el trabajo

$$
W=\frac{d U(x(t), a(t))}{d a} \circ d a
$$


Emulando la lógica de la termodinámica en la que la expresión para el trabajo termodinámico generalizado es la siguiente ecuación tiene la forma ${ }^{13}$

$$
d^{\prime} W=F d \lambda
$$

donde $F$ es una variable termodinámica que representa la fuerza macroscópica externa involucrada en la interacción y $d \lambda$ representa el cambio en la variable extensiva $\lambda$, para que esta expresión represente el trabajo debe de cumplir que el producto de una variable intensiva y una extensiva tenga dimensiones de energía y sea representativa de una interacción física, ejemplos de esto es el trabajo conocido de termodinámica $P d V$. Siguiendo esta lógica entonces se puede decir que el trabajo es el intercambio de energía generado por una fuerza que surge debido al control del sistema externo

$$
F=-\frac{\partial U(x(t), a(t))}{\partial a}
$$

Es una fuerza que ejerce la partícula sobre el sistema externo, de la misma manera pero con el signo contrario el sistema externo ejerce una fuerza sobre nuestra partícula. La multiplicación de esta fuerza por el desplazamiento da se entiende naturalmente como el trabajo hecho por el sistema externo sobre la partícula Browniana, y por lo tanto el parámetro externo $a$ es una variable extensiva. Es la manipulación del parámetro externo el que saca al sistema de su estado de equilibrio.

$$
W=\frac{\partial U}{\partial a} \cdot d a
$$

Cuando el trabajo es positivo implica que se está haciendo trabajo sobre la partícula Browniana; un trabajo negativo implica lo contrario, que la partícula Browniana hace trabajo sobre el sistema externo.

Aunque el parámetro externo sea determinista, es decir se puede controlar desde el experimento y no tiene un comportamiento fluctuante, el trabajo tiene una característica aleatoria debida al ruido del medio en el que se encuentra la partícula, esto implica que en cada realización del proceso el resultado del trabajo será diferente, sin embargo cuando el proceso es realizado durante un tiempo infinito, el resultado es independiente del protocolo con el que se varía el parámetro $a(t)$, solo depende del estado inicial y final del sistema.

Con la definición del trabajo 3.19 podemos reescribir la ecuación de balance de energía

$$
d E=d Q+d W
$$

esta ecuación es análoga a la primera ley de la termodinámica ya que ambas están basadas en el principio de conservación de la energía. La ecuación que hemos encontrado se cumple para cada realización de un proceso estocástico. Esta es la base de la termodinámica estocástica, el análisis de la energía sobre una realización de un proceso estocástico derivado de la ecuación de Langevin. 


\subsection{Entropía}

Para complementar el desarrollo teórico del análisis energético para sistemas pequeños que hemos realizado, surge el concepto de entropía para una sola trayectoria fluctuante. $\underline{30}$

Históricamente la definición de entropía surge del estudio de las máquinas térmicas como una medida que caracteriza la eficiencia con la cual la energía puede ser transformada en movimiento. El segundo postulado de la termodinámica nos lleva a entender que en un sistema termodinámico aislado, todo proceso que en él ocurra, irreversible o reversible, debe ser tal que la entropía del universo no disminuya; en el último caso, el cambio neto en la entropía del universo es igual a cero $\frac{13}{}$ Esto nos da una idea de la dirección en la que un proceso puede o no ocurrir en la naturaleza y un límite en la eficiencia.

¿Cómo podríamos aplicar la noción de la entropía a sistemas de tamaño micrométrico fuera del equilibrio? Para la entropía la interpretación se hace de manera diferente a lo que se realizó con la primera ley. Se hace con la definición de la entropía de Gibbs.

Al tener un sistema con un número enorme de partículas resulta imposible seguir el movimiento de cada una de ellas, Gibbs nos presenta en 1904 las bases para calcular propiedades macroscópicas de un sistema formado por un conjunto enorme de partículas, usando la definición de ensamble. El ensamble está compuesto por una gran cantidad de sistemas idénticos macroscópicamente pero cada uno se encuentra en una situación particular. Por ejemplo, en cada uno las partículas están en diferentes posiciones, o en diferentes microestados. ${ }^{4}$

Gibbs propone que la entropía de un sistema se puede ver como la propiedad de un ensamble de muchos sistemas idénticos. Para el sistema que nos interesa en esta tesis se ha propuesto en la literatura una extensión de la entropía de Gibbs donde se trabaja con una partícula ${ }^{30}$. En este caso, la densidad de probabilidad en el espacio fase $w(x(t), t)$, nos proporciona la probabilidad de que la partícula Browniana esté entre $x, x+d x$ al tiempo $t$. Asimismo,

$$
s(x(t), t)=-k_{B} \operatorname{Ln}[w(x(t), t)]
$$

nos da la entropía asociada con la trayectoria de la partícula y está evaluada con $x(t)$ a lo largo de la trayectoria para una realización del ruido. Esto conduce a que la entropía definida de esta forma sea una cantidad estocástica y por lo tanto podrá tomar todos los valores compatibles con las realizaciones que correspondan, es decir podremos tener una entropía negativa. El promedio sobre las realizaciones es lo que nos da un sentido físico claro. Dicho promedio también se puede realizar con la densidad de probabilidad, que en nuestro caso es solución de la ecuación de Fokker-Planck en la aproximación sobreamortiguada.

En los próximos capítulos veremos la manera de aplicar la termodinámica estocástica al modelo que propondremos, para esto primero debemos de determinar la ecuación de movimiento de la partícula Browniana. 


\section{Capítulo 4}

\section{Ecuación de Langevin y Fokker Planck}

Cuando una partícula Browniana se encuentra bajo la influencia de una fuerza externa su comportamiento se ve modificado. Nos interesa estudiar cuál es la modificación del comportamiento de la partícula Browniana cuando se encuentra bajo la influencia de las pinzas ópticas, desarrollaremos dos maneras para determinar la evolución temporal de la partícula, la ecuación de Langevin y la ecuación de Fokker-Planck.

Vimos en el modelo experimental que una partícula atrapada por las pinzas ópticas se puede asumir como una partícula atrapada en un potencial tipo oscilador armónico. Este es el primer paso a desarrollar para encontrar el comportamiento de la partícula Browniana y su densidad de probabilidad.

\subsection{Ecuación de Langevin para el oscilador ar- mónico}

Cuando la partícula Browniana se encuentra atrapada por las pinzas ópticas se dice que se encuentra dentro de un potencial tipo oscilador armónico, supondremos que nuestro modelo está en una dimensión,

$$
U(x, k)=\frac{1}{2} k x^{2}
$$

en donde el parámetro externo $a$ está dado por la rigidez del potencial $k$ que en este caso es constante. La fuerza derivada del potencial se ejerce sobre la partícula.

$$
F_{e x t}=-\frac{\partial U(x(t), k)}{\partial x}=-k x(t)
$$

La fuerza externa de las pinzas ópticas se incluye en la ecuación de Langevin.

$$
F=-\alpha v(t)+m \Gamma(t)+F_{e x t}=-\alpha v(t)+m \Gamma(t)-k x(t)
$$


La ecuación de Langevin en su forma completa por unidad de masa es

$$
\begin{aligned}
\dot{x} & =v(t) \\
\dot{v} & =-\frac{\alpha}{m} v(t)-\frac{k}{m} x+\Gamma(t)
\end{aligned}
$$

Renombramos $\frac{\alpha}{m}=\gamma$ de manera que podemos reescribir (4.5) como

$$
\dot{v}=-\gamma v(t)-\frac{k}{m} x(t)+\Gamma(t)
$$

El ruido del sistema tiene una forma Gaussiana y es conocido como ruido blanco $\Gamma(t)$, al obtener el promedio sobre un ensamble de muchas realizaciones del mismo proceso se encuentran las siguientes propiedades estadísticas:

$$
\begin{aligned}
\langle\Gamma(t)\rangle & =0 \\
\left\langle\Gamma(t) \Gamma\left(t^{\prime}\right)\right\rangle & =q \delta\left(t-t^{\prime}\right)
\end{aligned}
$$

$q$ en la ecuación (4.8), nos indica la intensidad del ruido que cuando el sistema se encuentra en equilibrio térmico con el medio en el que se encuentra se relaciona con la temperatura mediante el teorema de fluctuación disipación.

En esta tesis se trabajará con la aproximación sobre-amortiguada, de manera que la inercia del sistema es despreciable y la ecuación de Langevin se reduce a una sola que describe la dinámica del sistema.

$$
\dot{x}=-\frac{k}{\gamma m} x(t)+\frac{\Gamma(t)}{\gamma}
$$

La ecuación 4.9 es lineal diferencial ordinaria de primer orden, para resolver esta ecuación se puede usar el método del factor integrante:

$$
x(t)=x\left(t_{0}\right) e^{-\frac{k}{\gamma m}\left(t-t_{0}\right)}+\frac{1}{\gamma} \int_{t_{0}}^{t} \Gamma\left(t^{\prime}\right) e^{-\frac{k}{\gamma m}\left(t-t^{\prime}\right)} d t^{\prime}
$$

Al sacar el promedio de la posición sobre el ensamble, con las propiedades Gaussianas del ruido llegamos a la expresión que define el promedio de la posición de la partícula Browniana.

$$
\langle x(t)\rangle=x\left(t_{0}\right) e^{-\frac{k}{\gamma m}\left(t-t_{0}\right)}
$$

El comportamiento del promedio se puede observar en la imagen a) de la figura 4.1, esta gráfica es puramente esquemática para mostrar el relajamiento. Vemos que al transcurrir el tiempo el promedio del valor de la posición va disminuyendo hasta que finalmente adquiere el valor de cero. Esto significa que la partícula llega a "olvidar" el valor inicial de su posición. El tiempo $t$ que la velocidad tarda en llegar a un valor pequeño, comparado con el inicial, es el tiempo de relajación. Notamos que este tiempo depende de la rigidez del potencial, al aumentar el valor 
de la rigidez disminuye el tiempo de relajación.

Por otra parte, la correlación es

$$
\left\langle x\left(t_{1}\right) x\left(t_{2}\right)\right\rangle=x\left(t_{0}\right)^{2} e^{-\frac{k}{m \gamma}\left(t_{1}+t_{2}-2 t_{0}\right)}+\frac{q m}{2 \gamma k}\left(e^{-\frac{k}{m \gamma}\left|t_{1}-t_{2}\right|}-e^{-\frac{k}{m \gamma}\left(t_{1}+t_{2}-2 t_{0}\right)}\right)
$$

Para tiempos grandes, es independiente de la posición inicial y solo depende de la diferencia en el tiempo $\frac{q m}{2 \gamma k} e^{-\left(\frac{k}{m \gamma}\right)\left|t_{1}-t_{2}\right|}$, esto se nota en la figura b de [4.1], parece ser que la rigidez del potencial tiene el mismo efecto que tiene la friccion del medio, mientras más grande sea, se reduce la intensidad del movimiento.

a)

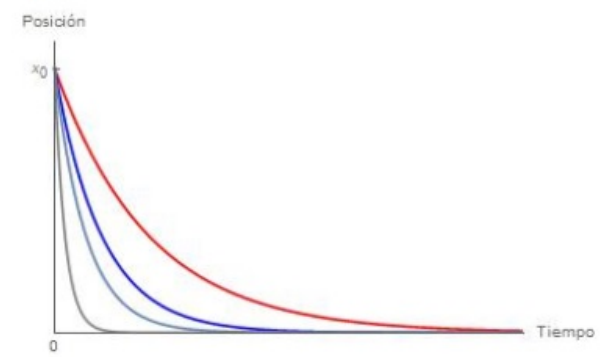

b)

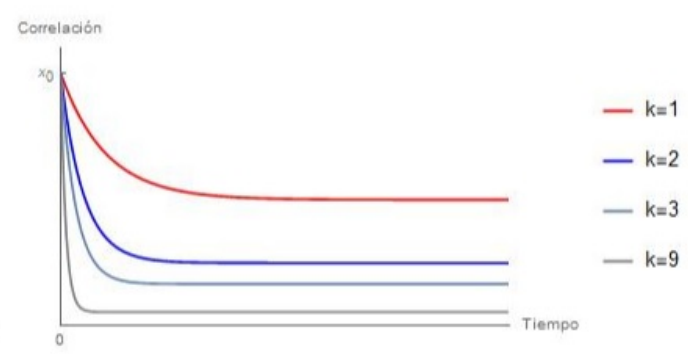

Figura 4.1: Del lado izquiero en la figura a se muestra la gráfica puramente cualitativa del comportamiento del promedio de la posición de la partícula, del lado derecho en la figura b se muestra la gráfica de la correlación de la posición.

La ecuación de Langevin nos indica el comportamiento de la partícula cuando es manipulada por las pinzas ópticas, esta es una manera de estudiar estos procesos, pero existen diversas formas como la ecuación de Boltzmann y la ecuación maestra que también describen los procesos estocásticos. Para los fines de esta tesis nos interesa por su equivalencia con la ecuación de Langevin y la información extra que contiene, estudiar la ecuación de Fokker-Planck.

\subsection{Ecuación de Fokker-Planck}

La ecuación de Fokker-Planck es una ecuación de evolución temporal de la densidad de probabilidad $w(x, t)$ para las variables fluctuantes, ésta nos indica la probabilidad de encontrar a la partícula en un cierto intervalo de posición $(x, x+d x)$ a un tiempo $t$, por lo que la ecuación nos indica cómo evoluciona dicha función de distribución. La forma general para desarrollar la ecuación de evolución temporal de la densidad de probabilidad es el desarrollo de Kramers Moyal.

$$
\frac{\partial w(\xi, t)}{\partial t}=\sum_{n=1}^{n=2}\left(-\frac{\partial}{\partial \xi}\right)^{n} D^{(n)}(\xi, t) w(\xi, t)
$$

En donde $D^{(n)}(\xi, t)$ son los coeficientes de Kramers-Moyal y se definen como la derivada de los cumulantes $k_{n}$ divididos por $n$ !, los cuales cumplen con $k_{1}=M_{1}$, 
$k_{2}=M_{2}-M_{1}^{2}, k_{3}=M_{3}-3 M_{1} M_{2}+2 M_{1}^{3}$ y los momentos $M_{n}$ están dados por $M_{n}=\left\langle\xi^{n}\right\rangle$ con $\xi$ una variable aleatoria, cuando el promedio del ruido es nulo, como lo es en este caso, los momentos y cumulantes coinciden.

$$
D^{(n)}(\xi)=\frac{1}{n !} \frac{d}{d t} M_{n}
$$

En el caso del ruido Gaussiano, por sus propiedades, solo los primeros dos términos de la serie son diferentes de cero, entonces:

$$
\frac{\partial w(\xi, t)}{\partial t}=\left[-\frac{\partial}{\partial \xi} D^{(1)}(\xi, t)+\frac{\partial^{2}}{\partial \xi^{2}} D^{(2)}(\xi, t)\right] w(\xi, t) .
$$

$D^{(1)}$ es llamado el coeficiente de deriva y $D^{(2)}$ es el coeficiente de difusión, estos coeficientes dependen también del tiempo. Matemáticamente es una ecuación diferencial parcial lineal de segundo orden de tipo parabólica, y se le conoce como la ecuación de Kolmogorov.27

Al resolver la ecuación de Fokker-Planck podemos obtener la función de distribución. Debido a que la aplicación de la ecuación de Fokker-Planck no está restringida al sistema cerca del equilibrio, podemos aplicarla a sistemas que se encuentran lejos del equilibrio. La ecuación de Fokker Planck no solo describe propiedades estacionarias, también describe la dinámica del sistema.

\subsubsection{Relación entre la ecuación de Langevin y la ecua- ción de Fokker-Planck}

La relación entre la ecuación de Fokker-Planck y la ecuación de Langevin está dada por los coeficientes de Kramers-Moyal, el de deriva y el de difusión. El proceso para pasar de Langevin a Fokker-Planck es el siguiente:

Proponemos como ejemplo una ecuación de Langevin en una dimensión en dónde $h(\xi, t)$ y $g(\xi, t)$ son funciones de la variable aleatoria y que en general no son lineales 27

$$
\dot{\xi}=h(\xi, t)+g(\xi, t) \Gamma
$$

De la ecuación de Langevin se pueden encontrar los coeficientes de KramersMoyal, el coficiente de deriva está determinado por

$$
\begin{aligned}
D^{(1)} & =\lim _{\tau \rightarrow 0} \frac{1}{\tau}\langle(\xi(t+\tau)-\langle\xi(t)\rangle)\rangle \\
& =h(\xi, t)+\frac{\partial g(\xi, t)}{\partial \xi} g(\xi, t)
\end{aligned}
$$

y el coeficiente de difusión es

$$
\begin{aligned}
D^{(2)} & =1 / 2 \lim _{\tau \rightarrow 0} \frac{1}{\tau}\left\langle(\xi(t+\tau)-\langle\xi(t)\rangle)^{2}\right\rangle \\
& =1 / 2 \lim _{\tau \rightarrow 0} \frac{1}{\tau}\left\langle\int_{t}^{t+\tau} g\left(x, t^{\prime}\right) \Gamma\left(t^{\prime}\right) d t^{\prime} \int_{t}^{t+\tau} g\left(x, t^{\prime \prime}\right) \Gamma\left(t^{\prime \prime}\right) d t^{\prime \prime}\right\rangle
\end{aligned}
$$


Comparando la ecuación de Langevin que propusimos 4.14 con la ecuación de Langevin cuando está influenciada por un potencial tipo oscilador armónico (4.24) encontramos que

$$
h(\xi, t)=-\frac{k}{m \gamma} \quad g(\xi, t)=\frac{1}{\gamma}
$$

Entonces

$$
D^{(1)}=-\frac{k x}{m \gamma}
$$

y para el coeficiente de difusión

$$
\begin{aligned}
D^{(2)} & =1 / 2 \lim _{\tau \rightarrow 0} \frac{1}{\tau}\left\langle\int_{t}^{t+\tau} \frac{1}{\gamma} \Gamma\left(t^{\prime}\right) d t^{\prime} \int_{t}^{t+\tau} \frac{1}{\gamma} \Gamma\left(t^{\prime \prime}\right) d t^{\prime \prime}\right\rangle \\
& =1 / 2 \lim _{\tau \rightarrow 0} \frac{1}{\tau} \frac{1}{\gamma^{2}} \int_{t}^{t+\tau} d t^{\prime} \int_{t}^{t+\tau}\left\langle\Gamma\left(t^{\prime}\right) \Gamma\left(t^{\prime \prime}\right)\right\rangle d t^{\prime \prime} \\
& =\frac{q}{2 \gamma^{2}}
\end{aligned}
$$

$\mathrm{Al}$ sustituir los valores de los coeficientes (4.18) y (4.21) en el desarrollo de Kramers-Moyal para el caso de ruido Gaussiano llegamos a la ecuación

$$
\frac{\partial w(x, t)}{\partial t}=\left[\frac{k}{m \gamma} \frac{\partial x}{\partial x}+\frac{q}{2 \gamma^{2}} \frac{\partial^{2}}{\partial x^{2}}\right] w(x, t)
$$

Esta es la ecuación de Fokker-Planck correspondiente a la ecuación de Langevin para una partícula Browniana en presencia de un potencial tipo oscilador armónico.

La teoría para determinar la dinámica de las partículas Brownianas ha sido ampliamente desarrollada y como hemos visto existen varios enfoques que tratan con ellos, el uso de cada enfoque depende del problema a manejar, en este caso la ecuación de Langevin y de Fokker-Planck serán la base de desarrollo para estudiar una partícula Browniana que pasa por diferentes procesos termodinámicos.

\subsection{El oscilador armónico con dependencia tem- poral}

Cuando el potencial en el que está atrapada la partícula Browniana es modificado externamente, se modifica el comportamiento de la partícula Browniana, y dependerá de la manera en la que es modificado en función del tiempo la trayectoria que observaremos.

Tomando en cuenta al sistema en una sola dimensión, el efecto de las pinzas ópticas por sí solas sobre la partícula es el de un potencial tipo oscilador armónico en el cual la partícula Browniana queda atrapada y puede manipularse mediante el parámetro externo que ahora tendrá una dependencia temporal $k(t)$.

$$
U(x, k(t))=\frac{1}{2} k(t) x^{2}(t)
$$


La manipulación de la rigidez de la trampa, $k(t)$, es debida al potencial externo $V_{k}$ que vimos en el modelo experimental y será necesario dejarlo expresado así hasta que se indique si este parámetro es constante o variable durante el proceso realizado.

La intensidad de ruido $q(t)$, nos indica la intensidad del ruido y debido al segundo voltaje aplicado en el experimento $V_{q}$ la intensidad del ruido es una cantidad controlable en el experimento de forma externa, por lo que en general tiene una dependencia temporal y no es aleatorio.

En la aproximación sobre-amortiguada llegamos el equivalente de la solución en equilibrio pero ahora con una dependencia temporal en $q(t)$ y $k(t)$

$$
\dot{x}=-\frac{k(t)}{\gamma m} x+\frac{\Gamma(t)}{\gamma}
$$

La solución a esta ecuación está dada por

$$
x(t)=x\left(t_{0}\right) e^{-\frac{1}{\gamma m} \int_{t_{0}}^{t} k\left(t^{\prime \prime \prime}\right) d t^{\prime \prime \prime}}+\frac{1}{\gamma} \int_{t_{0}}^{t} \Gamma\left(t^{\prime}\right) e^{-\frac{1}{\gamma m} \int_{t^{\prime}}^{t} k\left(t^{\prime \prime \prime}\right) d t^{\prime \prime \prime}} d t^{\prime}
$$

cuyo desarrollo se encuentra en el Apéndice B.

Para encontrar la función de distribución del sistema, que nos será de mucha ayuda más adelante, pasamos de la ecuación de Langevin (4.24) a la ecuación de Fokker-Planck. Como vimos en el capítulo anterior debemos de encontrar los coeficientes de deriva 4.16) y de difusión (4.17).

Los coeficientes son los mismos que encontramos para el oscilador armónico simple pero ahora muestran una dependencia temporal en la rigidez del potencial y en la intensidad del ruido. Tenemos que $h(\xi, t)=-\frac{k(t)}{m \gamma}$ y $g(\xi, t)=\frac{1}{\gamma}$ por lo que

$$
D^{(1)}=-\frac{k(t) x}{m \gamma}
$$

y el coeficiente de difusión se encuentra utlizando las propiedades del ruido Gaussiano manteniendo la dependencia temporal en la intensidad del ruido

$$
\begin{aligned}
D^{(2)} & =1 / 2 \lim _{\tau \rightarrow 0} \frac{1}{\tau}\left\langle\int_{t}^{t+\tau} \frac{1}{\gamma} \Gamma\left(t^{\prime}\right) d t^{\prime} \int_{t}^{t+\tau} \frac{1}{\gamma} \Gamma\left(t^{\prime \prime}\right) d t^{\prime \prime}\right\rangle \\
& =1 / 2 \lim _{\tau \rightarrow 0} \frac{1}{\tau} \frac{1}{\gamma^{2}} \int_{t}^{t+\tau} d t^{\prime} \int_{t}^{t+\tau}\left\langle\Gamma\left(t^{\prime}\right) \Gamma\left(t^{\prime \prime}\right)\right\rangle d t^{\prime \prime} \\
D^{2)} & =\frac{q(t)}{2 \gamma^{2}}
\end{aligned}
$$

Cuando el sistema se encuentra en equilibrio térmico con el ambiente y mantenemos la intensidad del ruido constante, existe una relación entre la intensidad del

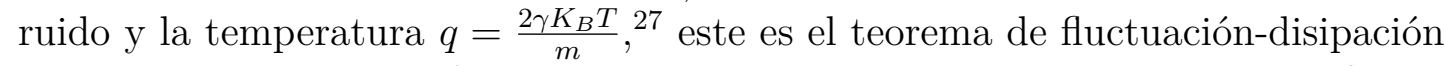
que no está garantizado fuera del equilibrio. Tomando esto en cuenta el coeficiente de difusión regresa al valor encontrado por Einstein que vimos en el primer 
capítulo.

$$
D^{(2)}=\frac{K_{B} T}{m \gamma}
$$

En nuestro caso el sistema no se encuentra en equilibrio con el medio debido a que las variaciones externas de los parámetros la sacan de este estado.

Con los coeficientes de difusión y de deriva al sustituirlos en el desarrollo de Kramers- Moyal llegamos a la ecuación de Fokker-Planck

$$
\frac{\partial w(x, t)}{\partial t}=\frac{k(t)}{m \gamma} \frac{\partial(x(t) w(x, t))}{\partial x}+\frac{q(t)}{2 \gamma^{2}} \frac{\partial^{2} w(x, t)}{\partial x^{2}}
$$

Esta ecuación nos indica la forma en la que varía en el tiempo la probabilidad de encontrar a la partícula en un intervalo $(x, x+d x)$ a un tiempo $t$.

Debido a que la ecuación de Langevin es una ecuación diferencial de primer orden, lineal y con ruido Gaussiano. La solución de la ecuación de Fokker-Planck es Gaussiana.

$$
w(x, t)=A(t) e^{-\epsilon(t) x^{2}}
$$

$A(t)=\sqrt{\frac{\epsilon(t)}{\pi}}$ es el coeficiente de normalización de la Gaussiana y $\epsilon(t)$ está relacionada con la varianza. Para que la distribución propuesta sea una solución debe de satisfacer

$$
\dot{\epsilon}=\frac{2 \epsilon(t)}{\gamma}\left[\frac{k(t)}{m}-\frac{q(t) \epsilon(t)}{\gamma}\right]
$$

La solución es más fácil de construir en términos de $\frac{1}{\epsilon(t)}$ y con una integración directa se obtiene:

$$
\frac{1}{\epsilon(t)}=\frac{1}{\epsilon\left(t_{0}\right)} e^{-\frac{2}{\gamma m} \int k\left(t^{\prime}\right) d t^{\prime}}+\frac{2}{\gamma^{2}} \int_{t_{0}}^{t} q\left(t^{\prime}\right) e^{-\frac{2}{\gamma m} \int_{t^{\prime}}^{t} k(t) d t^{\prime \prime}} d t^{\prime}
$$

Para completar la solución se necesita información de la manera en la que $k(t)$ y $q(t)$ dependen en el tiempo, esto nos lo da el protocolo. El protocolo se especificará en el próximo capítulo. 



\section{Capítulo 5}

\section{Ciclo de Stirling}

En la revolución industrial se desarrolló ampliamente la idea de construir máquinas que a partir de calor generaran trabajo, en un sentido amplio una máquina térmica es aquella que usa energía y parte de esa energía la devuelve como trabajo, las limitaciones de esta transferencia de energía se dan por el segundo postulado de la termodinámica. Aunque antes ya se tenían registro de prototipos de máquinas térmicas, no fue hasta 1698 que Thomas Savery ideó la manera de elevar grandes cantidades de agua utilizando calor.

Se comenzaron a utilizar de manera industrial máquinas para realizar esta labor. Varios prototipos de máquinas fueron diseñados para el uso en la industria minera para sacar agua de las minas; otro tipo de máquina de vapor se desarrolló para su aplicación en la industria ferrocarrilera. Desde ese entonces hasta nuestros tiempos se ha buscado la manera de hacer más eficientes las máquinas ${ }^{14}$.

\subsection{Ciclo de Stirling}

En 1816, Robert Stirling patentó lo que ahora conocemos como máquina de Stirling, se trata de una máquina térmica y fue la primera máquina ideada en la que la sustancia trabajaba en un circuito cerrado. El ciclo opera por una compresión y expansión cíclica de un fluido a diferentes temperaturas, está diseñada para que el gas se comprima en la parte fría de la máquina y se expanda en la caliente, como se muestra en la figura 5.1, el ciclo está constituido por dos procesos isotérmicos y dos procesos isocóricos, es un ciclo termodinámico idealizado, ya que es reversible, lo que implica que el intercambio de calor debe de ser perfecto (no hay pérdidas de energía) y el proceso debe de ser realizado cuasiestáticamente.

Consideramos un cilindro con dos pistones opuestos como se muestra en la figura 5.2. con un regenerador, una esponja térmica que absorbe y libera calor alternativamente, entre los pistones. El volumen entre el regenerador y el pistón izquierdo es el volumen de expansión y entre el regenerador y el pistón derecho es el de compresión. El volumen de expansión se mantiene a una temperatura máxima mientras que el volumen de compresión se mantiene a una temperatura mínima. Para comenzar con el ciclo asumimos que el pistón del espacio de compresión está en el extremo derecho y el pistón del espacio de expansión está cerca del regene- 


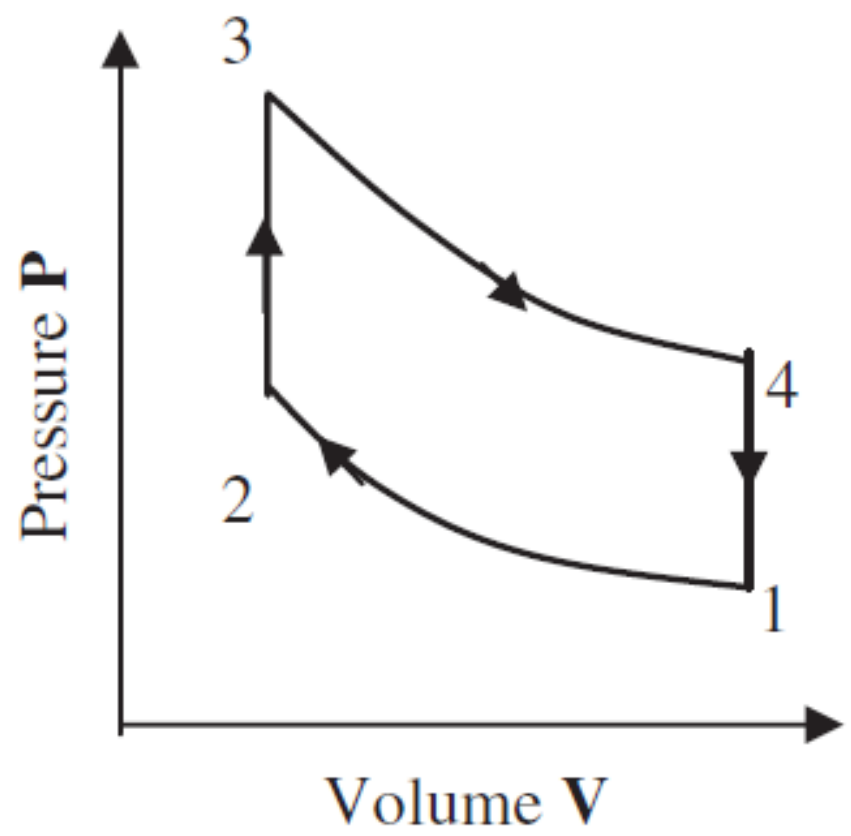

Figura 5.1: Ciclo de Stirling que consiste de cuatro procesos, la compresión y expansión isotérmica, y a volumen constante la adición y sustracción de calor.

rador, en este punto todo el fluido de trabajo está en el espacio de compresión a una temperatura mínima. El volumen de compresión está en su máximo y la presión y temperatura están en su mínimo.

Durante el primer proceso el pistón de compresión se mueve hacia el regenerador mientras que el pistón de expansión se mantiene estacionario. El fluido es comprimido, pasa de un volumen mínimo $v_{\min }$ a un volumen máximo $V_{\max }$ y la presión aumenta, la temperatura se mantiene constante en $T=T_{\min }$ debido al flujo de calor del espacio frío a los alrededores, tomando en cuenta que la máquina trabaja con un gas ideas y debido a que se encuentra en un proceso isotérmico la variación de la energía interna es cero y el calor por primera ley de la termodinámica es igual al trabajo

$$
\Delta Q=\Delta W=\int_{V_{\max }}^{V_{\min }} P d V=n R T_{\min } \operatorname{Ln}\left(\frac{V_{\min }}{V_{\max }}\right)<0
$$

en este caso se hace trabajo sobre el fluido para compensarlo el calor fluye a sus alrededores.

Durante el segundo proceso los dos pistones se mueven simultáneamente, el pistón de compresión se mueve hacia el regenerador y el de expansión se aleja de éste. Así el volumen entre los pistones se mantiene constante, $V=V_{1}$ mientras que el fluido es transferido del volumen de compresión al volumen de expansión, y pasa de una temperatura mínima $T_{\min }$ a la temperatura máxima $T_{\max }$. El aumento gradual de la temperatura causa un aumento en la presión, en este caso.

$$
\Delta W=0
$$




$$
\Delta Q=\Delta U=n c_{V} \Delta T=n c_{V}\left(T_{\max }-T_{\min }\right)
$$

En este caso el gas absorbe calor aumentando su energía interna y por lo tanto su temperatura.

Durante el tercer proceso el pistón de expansión continúa moviéndose lejos del regenerador mientras que el pistón de compresión se mantiene estacionario. Mientras sucede la expansión, la presión disminuye y el volumen aumenta de $V_{\min }$ a $V_{\max }$. La temperatura se mantiene constante en $T=T_{\max }$ debido al calor absorbido del baño térmico caliente. Entonces

$$
\Delta Q=\Delta W=\int_{V_{\min }}^{V_{\max }} n R T_{\max } \operatorname{Ln}\left(\frac{V_{\max }}{V_{\min }}\right)
$$

el gas realiza trabajo mientras absorbe calor.

En el último proceso los dos pistones se mueven simultáneamente para transferir el fluido al espacio de compresión manteniendo el volumen constante en $V_{2}$. Durante el flujo de este fluido el calor es transferido del fluido al regenerador, reduciendo la temperatura de $T_{\max }$ hasta llegar al mínimo $T_{\min }$.

$$
\begin{aligned}
\Delta W & =0 \\
\Delta Q & =\Delta U=n c_{V}\left(T_{\min }-T_{\max }\right)
\end{aligned}
$$

El gas ideal cede calor disminuyendo su energía interna, el resultado del proceso es la conversión de calor en trabajo.

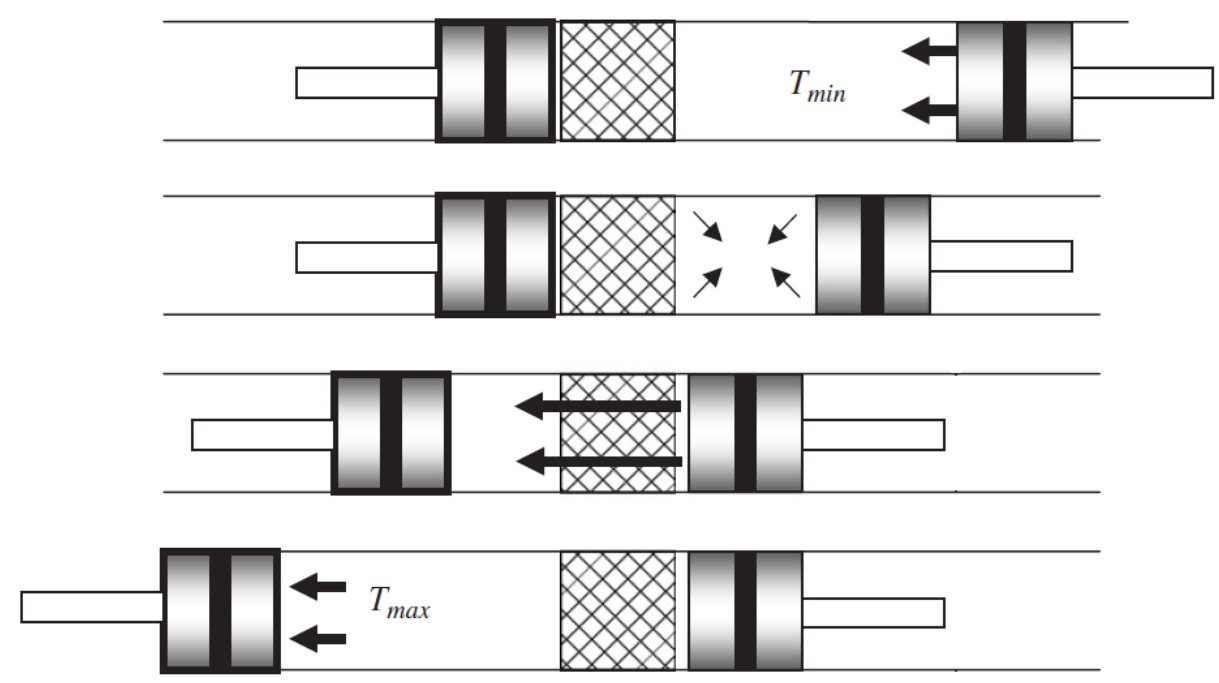

Figura 5.2: Arreglo de pistones de la máquina

El ciclo de Stirling tiene una eficiencia calorífica grande, es capaz de alcanzar la eficiencia del ciclo de Carnot solo en el caso ideal. Dado que el motor Stirling tiene la habilidad de utilizar fuentes de energía alternativas y renovables puede ser muy importante en industrias que busquen utilizar energías alternativas y eficientes, el estudio de este tipo de máquinas sigue vigente. 
Nos interesa ampliar los estudios de termodinámica de sistemas macroscópicos a sistemas pequeños del orden de micras para el caso de una sola partícula Browniana.

En el caso micrométrico tenemos una partícula controlada por las pinzas ópticas y dos voltajes externos $V_{k}$ y $V_{q}$ que nos permite generar procesos con los cuales se modifica a la partícula Browniana. La variación en el volumen se puede interpretar como un cambio en la rigidez del potencial armónico generado por las pinzas ópticas. Esta variación en el potencial puede generar que la partícula tenga más libertad para moverse cuando la rigidez del potencial es pequeña o puede limitar su movimiento cuando la rigidez del potencial es grande, como se puede observar en la figura 5.3

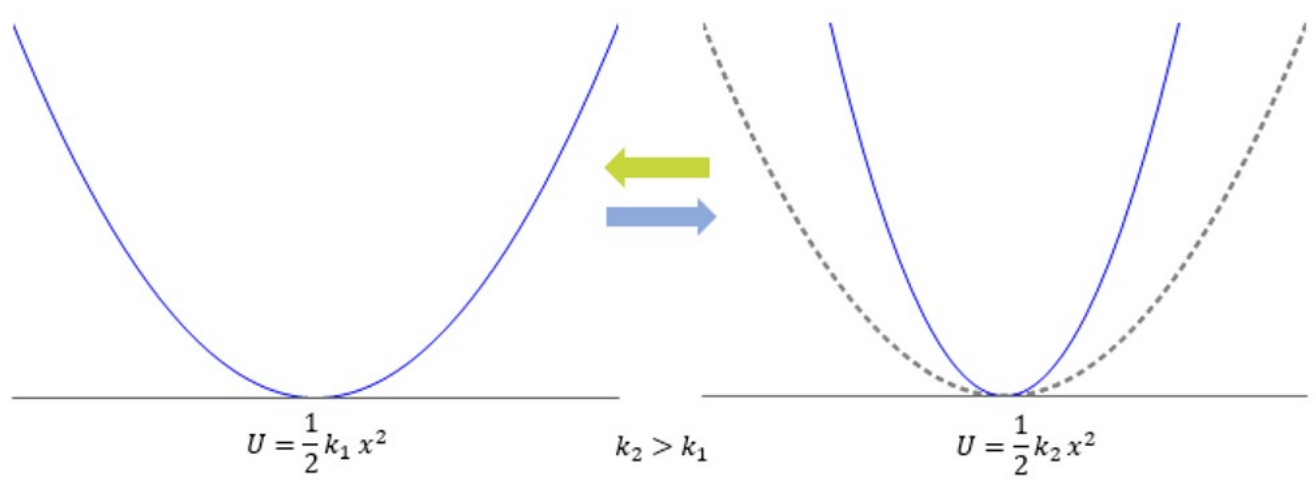

Figura 5.3: Se muestra de manera gráfica el cambio que se genera cuando se varía la rigidez del potencial con $k_{2}=2 k_{1}$.

La temperatura en el caso micrométrico no se puede definir a menos que el sistema se encuentre en equilibrio con el medio en el que se encuentra, de otra manera se deja indicado que es proporcional al ruido que siente el sistema. Un proceso isotérmico será en nuestro caso un proceso que sucede a una intensidad de ruido constante, es decir, $q(t)$ no variará en el tiempo, y los procesos isocóricos, es decir, a volumen constante, se realizan manteniendo invariante la rigidez del potencial armónico.

\subsection{Ciclo de Stirling en cuasi-equilibrio}

Una primera aproximación del problema que ya se ha llevado a cabo en varios artículos 1] 21]29]40 es considerar que los procesos termodinámicos son llevados por estados de cuasi-equilibrio. En estos procesos los parámetros de control cambian de forma mucho más lenta que los tiempos de relajación del sistema, así pasa por estados de cuasi-equilibrio, llegando a un estado final en equilibrio. De esta manera el resultado es independiente del camino por el que se lleva acabo el proceso.

Para un proceso de este tipo se utiliza la densidad de puntos de un ensamble canónico en el espacio fase y se describe con la distribución de Gibbs sobre el 
proceso. Para encontrar la distribución debemos de determinar la hamiltoniana del sistema, en este caso se toma en cuenta el potencial de las pinzas ópticas

$$
H(x(t), v(t), t)=\frac{1}{2} k(t) x(t)^{2}+\frac{1}{2} m v(t)^{2}
$$

Ya que el estudio en esta tesis se realiza en el caso sobre-amortiguado, la hamiltoniana correspondiente es

$$
H\left(x_{t}, t\right)=\frac{1}{2} k(t) x(t)^{2}
$$

y nos indica que al tiempo $t$ el sistema está descrito por un ensamble Canónico, entonces la densidad de probabilidad de encontrar a la partícula browniana con una posición $x$ a un tiempo $t$ es

$$
\rho\left(x_{t}, t\right)=\frac{e^{-\beta_{t} H\left(x_{t}, t\right)}}{Z_{t}}=\frac{e^{-\frac{\beta_{t} k_{t} x_{t}^{2}}{2}}}{Z_{t}}
$$

En donde

$$
\beta(t)=\frac{1}{k_{B} T(t)}
$$

y $Z_{t}$ es la función de partición en el ensamble canónico

$$
Z_{t}=\int d x e^{-\beta_{t} H(x(t), t)}=\sqrt{\frac{2 \pi k_{B} T(t)}{k(t)}}
$$

Sustituyendo la función de partición y el valor de $\beta(t)$ encontramos la densidad de probabilidad

$$
\rho(x, t)=\sqrt{\frac{k(t)}{2 \pi k_{B} T(t)}} e^{-\frac{\beta(t) k(t) x(t)^{2}}{2}}
$$

La densidad de probabilidad $\rho(x, t)$ la podemos comparar con la densidad de probabilidad de la solución general de Fokker-Planck (4.31) al hacer esto hallamos el valor de $\epsilon(t)$ (4.33) en el tratamiento de cuasi-equilibrio.

$$
\sqrt{\frac{k(t)}{2 \pi k_{B} T(t)}} e^{-\frac{k(t) x(t)^{2}}{2 k_{B} T(t)}}=\sqrt{\frac{\epsilon(t)}{\pi}} e^{-\epsilon(t) x^{2}}
$$

notamos la existencia una relación entre $\epsilon(t)$ con $k(t)$ y la temperatura del medio que lo rodea

$$
\epsilon(t)=\frac{k(t)}{2 k_{B} T(t)}
$$

Por otro lado $\epsilon(t)$ en equilibrio se puede derivar de la ecuación (4.32), ya que en este caso debe de cumplir que no existe un cambio en el tiempo sobre esta variable 


$$
\dot{\epsilon}(t)=\frac{2 \epsilon(t)}{\gamma}\left[\frac{k(t)}{m}-\frac{q(t) \epsilon(t)}{\gamma}\right]=0
$$

por lo tanto

$$
\epsilon(t)=\frac{\gamma k(t)}{m q(t)}
$$

Al sustituir el valor de $\epsilon(t)$ de la ecuación (5.13) en la ecuación (5.15) encontramos la relación que existe entre la intensidad del ruido con la temperatura que solo se cumple cuando el sistema se encuentra en equilibrio.

$$
q(t)=\frac{\gamma}{m} 2 k_{B} T(t)
$$

Nos regresa a la relación de fluctuación-disipación pero ahora para el cuasiequilibrio.

Reescribimos la función de partición (5.10) en términos de la intensidad del ruido y la rigidez de la trampa.

$$
Z(t)=\sqrt{\frac{\pi m q(t)}{\gamma k(t)}}
$$

La función de partición nos permite encontrar la ecuación de estado del sistema por su relación con la energía libre

$$
F(q, k)=-\frac{m q(t)}{2 \gamma} \operatorname{Ln}(z(q, k))=-\frac{m q(t)}{2 \gamma} \operatorname{Ln}\left(\sqrt{\frac{\pi m q(t)}{\gamma k(t)}}\right)
$$

La ecuación de estado del sistema, en termodinámica clásica está dada por $d F(T, v)=$ $-S d T-p d v$ al hacer el equivalente a sistemas pequeños definimos una función $\Phi$ semejante a la presión y llegamos a la siguiente relación.

$$
d F(q, k)=-\frac{m}{2 \gamma k_{B}} S d q+\Phi d k
$$

Partiendo de esta suposición, al utilizar la función de partición (5.17) y la energía libre (5.18) podemos obtener la ecuación de estado de nuestro sistema y la entropía

$$
\begin{aligned}
\Phi_{k} & =\left(\frac{\partial F}{\partial k}\right)_{q}=\frac{m q(t)}{4 \gamma k(t)} \\
S_{\text {efectiva }} & =\frac{m S}{2 \gamma k_{B}}=-\left(\frac{\partial F}{\partial q}\right)_{k}=\frac{m}{2 \gamma}\left(\operatorname{Ln} \sqrt{\frac{\pi m q(t)}{\gamma k(t)}}+1\right)
\end{aligned}
$$

Vemos que $\Phi$ está relacionada directamente con $q$ y es inversamente proporcional a $k$, lo cual es semejante al comportamiento de un gas ideal en donde $q$ juega el papel de la temperatura y $k$ del volumen. Siguiendo este enfoque, el trabajo está dado por la expresión

$$
d W=\frac{m q(t)}{4 \gamma k(t)} d k(t)
$$


La convención de signos utilizada es la siguiente: trabajo positivo implica que se realiza trabajo sobre la partícula y si es negativo indica que la partícula realiza trabajo sobre sus alrededores.

Además, con la relación entre la energía interna y la energía libre

$$
U=F+T S
$$

obtenemos la expresión para la energía interna.

$$
\begin{aligned}
U & =F+\frac{m q(t)}{2 \gamma} S \\
& =-\frac{m q(t)}{2 \gamma} \operatorname{Ln}\left(\sqrt{\frac{\pi m q(t)}{\gamma k(t)}}\right)+\frac{m q(t)}{2 \gamma}\left(\operatorname{Ln} \sqrt{\frac{\pi m q(t)}{\gamma k(t)}}+1\right) \\
& =\frac{m q(t)}{2 \gamma}
\end{aligned}
$$

El cambio en la energía interna

$$
\Delta U=\Delta Q+W
$$

únicamente depende de la intensidad del ruido, en el caso del calor, un calor positivo implica un flujo de energía del medio a la partícula, es calor absorbido y si es negativo es calor cedido. Tomando todo esto en cuenta podemos proponer el ciclo y calcular sus variables termodinámicas.

Para el ciclo Stirling tenemos dos procesos a $q$ constante y dos procesos a $k$ constante. Usando la ecuación de estado 5.20 podemos determinar cada uno de los procesos las curvas a $q$ constante corresponden a hipérbolas que se encuentran en función de $k$ y rectas para $k$ constante. Esto significa que en el espacio de las variables $(q, k)$ la forma del ciclo es muy semejante a la del ciclo Stirling con un gas ideal como se muestra en la figura 5.4 .

El primer proceso comienza en el punto $\left(k_{2}, q_{2}\right)$. Se trata de una disminución del potencial $k(t)$ el cual va de $k_{2}$ a $k_{1}$ siendo $k_{2}>k_{1}$; la disminución del potencial corresponde a una disminución en el atrapamiento de la partícula Broniana, esto implica que posibilita más su movimiento, el proceso sucede a una intensidad de ruido grande $q_{2}$. En este caso el trabajo está dado por

$$
\Delta W_{1 \rightarrow 2}=\frac{\gamma q_{2}}{4 \gamma} \int_{k_{2}}^{k_{1}} \frac{d k}{k}=-\frac{m q_{2}}{4 \gamma} \operatorname{Ln}\left(\frac{k_{2}}{k_{1}}\right)
$$

ya que el proceso se realiza manteniendo constante la intensidad del ruido, el cambio en la energía interna 5.23 es cero y por lo tanto

$$
\Delta W_{1 \rightarrow 2}=-\Delta Q
$$

Entonces

$$
\Delta Q_{1 \rightarrow 2}=\frac{m q_{2}}{4 \gamma} \operatorname{Ln}\left(\frac{k_{2}}{k_{1}}\right)
$$




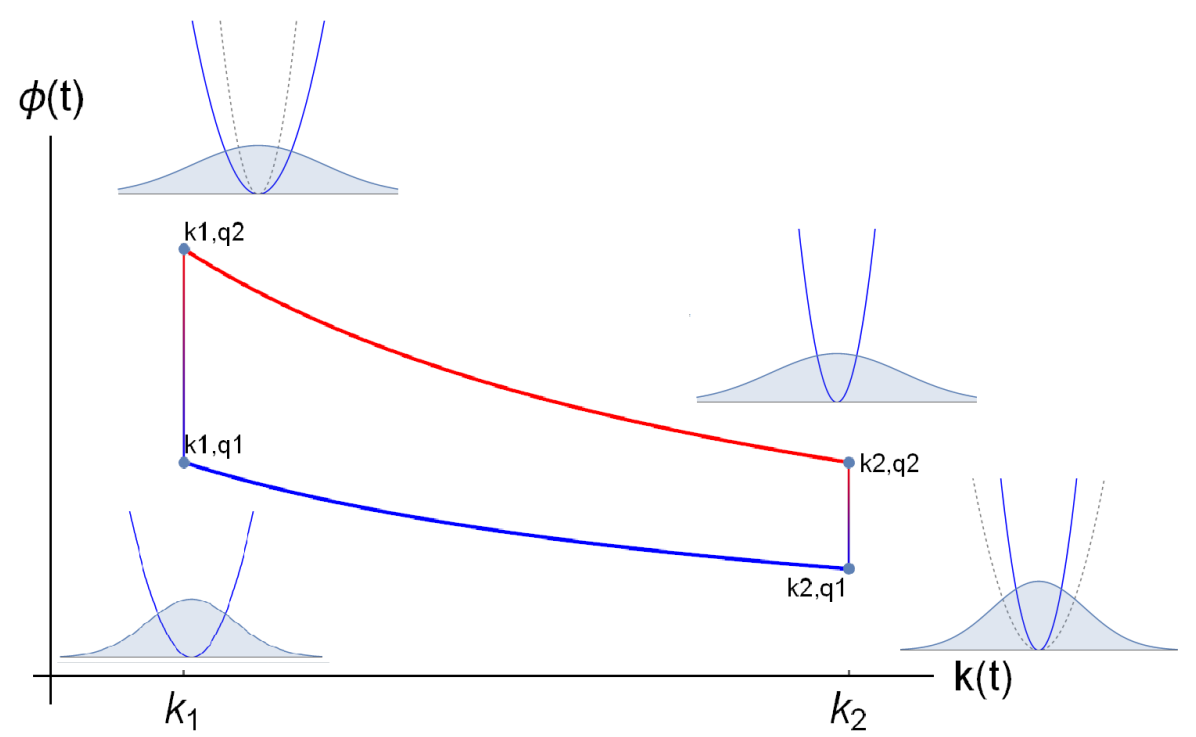

Figura 5.4: Cilo de Stirling Browniano en el espacio $\Phi, k$ en donde las curvas del espacio se generan a partir de la ecuación de estado 5.20.

Ya que $k_{2}>k_{1}$, el trabajo en este caso es una cantidad negativa y el calor es positivo, lo cual implica que el flujo de energía va del medio a la partícula, mientras tanto la partícula realiza trabajo sobre sus alrededores.

El segundo proceso, que comienza en el punto $\left(k_{1}, q_{2}\right)$ es la disminución en la intensidad del ruido que va de $q=q_{2}$ a $q=q_{1}$, siendo $q_{2}>q_{1}$ mientras que la dureza del potencial se mantiene constante con $k=k_{1}$. En este caso debido a que $k$ permanece constante, no existe trabajo y por lo tanto

$$
\begin{aligned}
\Delta W_{2 \rightarrow 3} & =0 \\
\Delta U_{2 \rightarrow 3} & =\Delta Q=\frac{m}{2 \gamma}\left(q_{1}-q_{2}\right)
\end{aligned}
$$

y ya que $q_{2}>q_{1}$, el calor es negativo. Esto implica que durante este proceso se libera calor al medio.

El tercer proceso que comienza en el punto $\left(k_{1}, q_{1}\right)$ es el aumento en el potencial que pasa de $k=k_{1}$ a $k=k_{2}$ por lo tanto la partícula tendrá un movimiento más restringido con una intensidad de ruido constante $q=q_{1}$. En este caso el trabajo es

$$
\Delta W_{3 \rightarrow 4}=\frac{m q_{1}}{2 \gamma} \operatorname{Ln}\left(\frac{k_{2}}{k_{1}}\right)
$$

y en este proceso no existe un intercambio en la energía interna ya que solo depende de la intensidad del ruido, debido a esto podemos expresar el calor como el negativo del trabajo

$$
\Delta Q_{3 \rightarrow 4}=-\frac{m q_{1}}{2 \gamma} \operatorname{Ln}\left(\frac{k_{2}}{k_{1}}\right)
$$

Entonces, vemos que se está realizando trabajo en el sistema mientras que el calor fluye de la partícula a sus alrededores. 
El último proceso que cierra el ciclo, comienza en el punto $\left(k_{2}, q_{1}\right)$, es el aumento en la intensidad de ruido que pasa de $q=q_{1}$ a $q=q_{2}$ mientras se mantiene un potencial constate con una rigidez $k=k_{2}$. En este caso no existe trabajo

$$
\Delta W_{4 \rightarrow 1}=0
$$

y el calor es igual al cambio en la energía interna.

$$
\Delta U_{4 \rightarrow 1}=\Delta Q_{4 \rightarrow 1}=\frac{m}{2 \gamma}\left(q_{2}-q_{1}\right)
$$

El cambio es positivo por lo que se está absorbiendo calor de los alrededores.

Finalmente la eficiencia de la máquina está determinada por el cambio total del trabajo sobre la cantidad de calor absorbida por el sistema.

$$
\eta=\frac{W_{\text {total }}}{Q_{a}}=\frac{\frac{m}{2 \gamma}\left(q_{2}-q_{1}\right) \operatorname{Ln}\left(\frac{k_{2}}{k_{1}}\right)}{\frac{m q_{2}}{2 \gamma} \operatorname{Ln}\left(\frac{k_{2}}{k_{1}}\right)}=1-\frac{q_{1}}{q_{2}}
$$

que guarda una gran similitud con el caso ideal.

En resumen los valores de las cantidades termodinámicas se pueden ver en el cuadro 5.1 .

\begin{tabular}{||c|c|c||}
\hline Proceso & $\langle\Delta W\rangle$ & $\langle\Delta Q\rangle$ \\
\hline \hline Disminución de $k_{2} \rightarrow k_{1} \operatorname{con} q(t)=q_{2}$ & $-\frac{m q_{2}}{4 \gamma} \operatorname{Ln}\left(\frac{k_{2}}{k_{1}}\right)$ & $\frac{m q_{2}}{4 \gamma} \operatorname{Ln}\left(\frac{k_{2}}{k_{1}}\right)$ \\
\hline Disminución de $q_{2} \rightarrow q_{1} \operatorname{con} k(t)=k_{1}$ & 0 & $\frac{m}{2 \gamma}\left(q_{1}-q_{2}\right)$ \\
\hline Aumento de $k_{1} \rightarrow k_{2}$ & $\frac{m q_{1}}{2 \gamma} \operatorname{Ln}\left(\frac{k_{2}}{k_{1}}\right)$ & $-\frac{m q_{1}}{2 \gamma} \operatorname{Ln}\left(\frac{k_{2}}{k_{1}}\right)$ \\
\hline Aumento de $q_{1} \rightarrow q_{2}$ & 0 & $\frac{m}{2 \gamma}\left(q_{2}-q_{1}\right)$ \\
\hline
\end{tabular}

Cuadro 5.1: Tabla con los valores del calor y el trabajo en cada uno de los procesos del ciclo de Stirling.

Estudiamos al sistema en un estado de semi-equilibrio, el resultado de este estudio es muy vago ya que al hacerlo no se están tomando en cuenta las fluctuaciones del medio, el resultado al que se llega es muy parecido al ciclo de Stirling que conocemos en la termodinámica clásica. El sistema trabaja a dos intensidades de ruido diferente, en el proceso la energía es transferida a la partícula, es decir, absorbe calor para transformar una parte de esta energía en trabajo.

Para determinar la solución completa en el caso de estudio de esta tesis es necesario plantear el proceso con el que se va a modificar a la partícula Browniana. En el próximo capítulo plantearemos el proceso para realizar un ciclo de Stirling. 



\section{Capítulo 6}

\section{Ciclo de Stirling Browniano}

El interés principal de esta tesis es estudiar los efectos de las fluctuaciones del medio que sienten partículas de tamaño micrométricas o más pequeñas. Se espera que aplicando de una manera adecuada la termodinámica estocástica a los sistemas micrométricos el promedio de las cantidades termodinámicas tales como el calor, el trabajo, la entropía y la eficiencia se vean modificadas de su caso de cuasi-equilibrio. En un sistema fuera de equilibrio es importante tomar en cuenta que el ciclo se realiza durante un tiempo finito de una cierta manera, el protocolo define cómo se van variando los parámetros externos, la rigidez de la trampa $k(t)$ y la intensidad del ruido $q(t)$ como función del tiempo. A partir de ello se encuentra la solución a la ecuación de Langevin que nos muestra el comportamiento de la partícula y la solución a la ecuación de Fokker-Planck que nos indica la probabilidad de encontrar a la partícula en $x+\Delta x$ a un tiempo $t$ para cada proceso del ciclo.

En términos de dicha distribución de probabilidad se encontrarán los promedios de las cantidades termodinámicas, trabajo, calor y entropía definidas en el capítulo 3 para la partícula Browniana en un tiempo finito. Además, podremos determinar qué tanto se diferencia del caso en cuasi-equilibrio y comprobaremos que en el caso cuasiestático las soluciones regresan a lo que estudiamos del ciclo en cuasiequilibrio.

\subsection{Protocolo}

En el modelo experimental desarrollado en el capítulo 1 explicamos que de forma experimental se pueden modificar externamente la intensidad del ruido $q(t)$ gracias a un voltaje externo $V_{q}$, y la rigidez de la trampa $k(t)$ gracias al voltaje externo $V_{k}$, esto nos da la libertad de proponer un protocolo que nos indique la forma en la que ambos parámetros cambian en el tiempo para poder realizar procesos termodinámicos. Como hemos visto la intensidad del ruido está relacionada con la temperatura y la rigidez de la trampa con el volumen.

Proponemos un protocolo del ciclo de Stirling en el que $q(t)$ y $k(t)$ varían linealmente en el tiempo para realizar dos procesos isotérmicos y dos isocóricos, el ciclo completo se realiza en un tiempo total $\tau$ y cada uno de sus cuatro procesos se realizarán en un tiempo $\frac{\tau}{4}$. 
Antes de comenzar con los cálculos proponemos las siguientes variables adimensionales, el detalle de este análisis se encuentra en el apéndice A.

$$
\begin{aligned}
\alpha & =\frac{k_{1} \tau}{m \gamma} \\
\triangle k & =\frac{k_{2}-k_{1}}{k_{1}} \\
\triangle q & =\frac{q_{2}-q_{1}}{q_{1}}
\end{aligned}
$$

$\mathrm{Al}$ definir estas variables podremos escribir las ecuaciones de manera adimensional, pero más allá de eso notamos que con las variables adimensionales podemos extraer más información, $\alpha$ mide el cociente de los tiempos característicos en el sistema y ello permite el análisis de resultados, $\tau$ es el tiempo del ciclo, mientras que $\frac{k_{1}}{m \gamma}$ tiene unidades de frecuecia, es un tiempo de relajación característico del sistema. $\triangle k$ y $\triangle q$ son cocientes que nos determinan qué tanto se varían externamente la rigidez de la trampa y la intensidad del ruido con respecto a un estado base. Por ejemplo, cuando se varía mucho la rigidez de la trampa existe un cambio muy grande entre $k_{1}$ y $k_{2}, \triangle k$ nos muestra esta diferencia comparándola con $k_{1}$ de manera adimensional. A partir de este momento se reescribirán las expresiones en función de las variables adimensionales.

En el primer proceso a intensidad de ruido constante, tenemos una disminución lineal de la rigidez del potencial, de $k_{2}$ a $k_{1}$, esto sólo es válido para $0 \leq t \leq \frac{\tau}{4}$

$$
\begin{aligned}
& k_{1 \rightarrow 2}(t)=\frac{k_{1}-k_{2}}{\tau} 4 t+k_{2}=k_{1}\left(1+\Delta k-\frac{4 t \Delta k}{\tau}\right) \\
& q_{1 \rightarrow 2}(t)=q_{2}=q_{1}(1+\Delta q)
\end{aligned}
$$

El segundo proceso con rigidez de la trampa óptica constante se realiza linealmente la disminución del ruido durante un tiempo finito que sólo es válido para $\frac{\tau}{4} \leq t \leq \frac{2 \tau}{4}$.

$$
\begin{aligned}
& k_{2 \rightarrow 3}(t)=k_{1} \\
& q_{2 \rightarrow 3}(t)=\frac{q_{1}-q_{2}}{\tau} 4\left(t-\frac{\tau}{4}\right)+q_{2}=q_{1}\left(1+\Delta q\left(2-\frac{4 t}{\tau}\right)\right)
\end{aligned}
$$

El tercer proceso es un aumento en la rigidez del potencial, a una intensidad de ruido mínima y constante válida para un intervalo de tiempo que va de $\frac{2 \tau}{4} \leq t \leq$ $\frac{3 \tau}{4}$

$$
\begin{aligned}
& k_{3 \rightarrow 4}(t)=\frac{k_{2}-k_{1}}{\tau} 4\left(t-\frac{2 \tau}{4}\right)+k_{1}=k_{1}\left(1+\Delta k\left(-2+\frac{4 t}{\tau}\right)\right) \\
& q_{3 \rightarrow 4}(t)=q_{1}
\end{aligned}
$$


El último proceso que cierra el ciclo sucede para $\frac{3 \tau}{4} \leq t \leq \tau$. Se trata de un aumento lineal en la intensidad del ruido con una rigidez de potencial constate

$$
\begin{aligned}
& k_{4 \rightarrow 1}(t)=k_{2}=k_{1}(1+\Delta k) \\
& q_{4 \rightarrow 1}(t)=\frac{q_{2}-q_{1}}{\tau} 4\left(t-\frac{3 \tau}{4}\right)+q_{1}=q_{1}\left(1+\Delta q\left(-3+\frac{4 t}{\tau}\right)\right)
\end{aligned}
$$

La dependencia temporal del protocolo la podemos observar en la figura 6.1. La
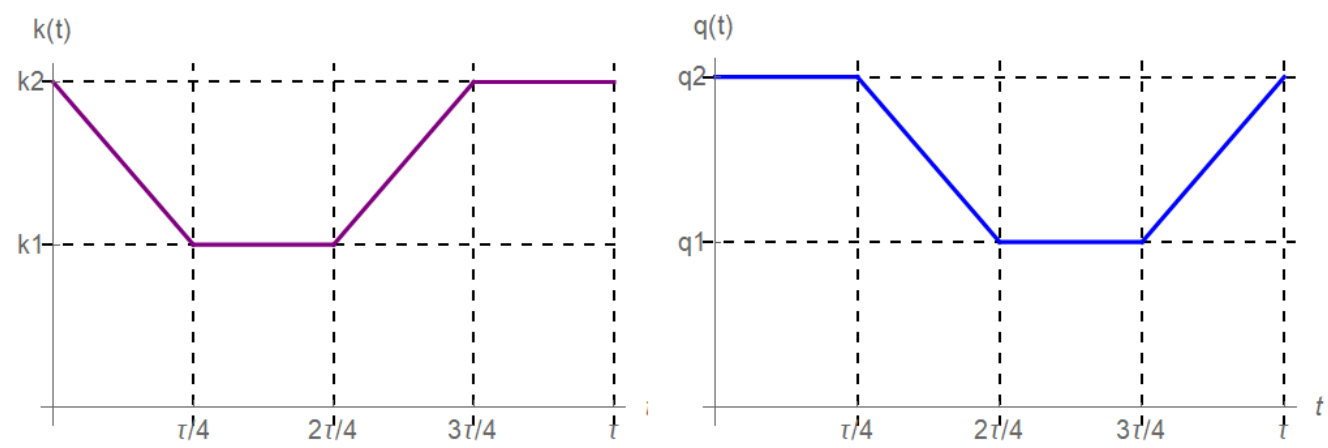

Figura 6.1: Gráficas realizadas con el protocolo arriba indicado. En la figura derecha se muestra el protocolo para el cambio en la intensidad del ruido $q(t)$ en función del tiempo $t$, en donde $q_{2}=2 q_{1}$. En la figura izquierda se muestra el protocolo para el cambio de la rigidez del potencial $k(t)$ en función del tiempo $t$, en el cual se toma en cuenta que $k_{2}=2 k_{1}$.

manera en que varían las variables externas, el cambio en la intensidad del ruido $q(t)$ y el cambio en la rigidez del potencial $k(t)$, con el protocolo propuesto en función del tiempo nos da la pauta para comenzar el análisis de nuestro modelo de un ciclo de Stirling realizado por una partícula Browniana en un tiempo finito.

\subsection{Ecuación de Langevin en el ciclo}

Para estudiar un sistema Browniano nos hemos enfocado en dos procedimientos distintos que son totalmente equivalentes, por un lado, la ecuación de Langevin determina la ecuación de evolución estocástica de la partícula Browniana y por otro lado la ecuación de Fokker-Planck nos indica la evolución temporal de la distribución de probabilidad.

La solución a la ecuación de Langevin ya fue encontrada parcialmente en (4.25). Es a partir de esta ecuación que introducimos el protocolo propuesto de nuestro modelo, de esta manera para cada uno de los procesos termodinámicos por los que pasa la partícula podremos determinar las soluciones estocásticas de la ecuación de Langevin. Con lo cual obtendremos la posición de la partícula en un instante y su trayectoria.

$$
x(t)=x\left(t_{0}\right) e^{-\frac{1}{\gamma m} \int_{t_{0}}^{t} k\left(t^{\prime \prime \prime}\right) d t^{\prime \prime \prime}}+\frac{1}{\gamma} \int_{t_{0}}^{t} \Gamma\left(t^{\prime}\right) e^{-\frac{1}{\gamma m} \int_{t^{\prime}}^{t} k\left(t^{\prime \prime \prime}\right) d t^{\prime \prime \prime}} d t^{\prime}
$$


El promedio y la correlación encontrados sobre un ensamble de muchas realizaciones se encuentra tomando en cuenta las propiedades del ruido Gaussiano

$$
\begin{gathered}
\langle\Gamma(t)\rangle=0 \\
\left\langle\Gamma(t) \Gamma\left(t^{\prime}\right)\right\rangle=q\left(t^{\prime}\right) \delta\left(t-t^{\prime}\right)
\end{gathered}
$$

El promedio está dado por la siguiente expresión.

$$
\langle x(t)\rangle=x\left(t_{0}\right) e^{-\frac{1}{\gamma m} \int_{t_{0}}^{t} k\left(t^{\prime \prime \prime}\right) d t^{\prime \prime \prime}}
$$

y la función de correlación está dada por

$$
\begin{aligned}
\left\langle x\left(t_{1}\right) x\left(t_{2}\right)\right\rangle & =\left\langle x\left(t_{1}\right)\right\rangle\left\langle x\left(t_{2}\right)\right\rangle \\
& +\frac{1}{\gamma^{2}} \int_{t_{0}}^{\left(t_{1}, t_{2}\right)} d t^{\prime}\left(q\left(t^{\prime}\right) e^{-\frac{1}{\gamma m}\left(\int_{t^{\prime}}^{t_{1}} k\left(t^{\prime \prime}\right) d t^{\prime \prime}+\int_{t^{\prime}}^{t_{2}} k\left(t^{\prime \prime}\right) d t^{\prime \prime}\right)}\right)
\end{aligned}
$$

El límite de integración es $t_{1}$ o $t_{2}$ dependiendo de cuál sea el menor. A partir de estas soluciones, tomando en cuenta una condición inicial para la posición y los protocolos en función de las variables adimensionales con los que cambia $k\left(t^{\prime \prime \prime}\right)$ y $q\left(t^{\prime}\right)$ se pueden encontrar la posición de la partícula Browniana a un tiempo $t$ su promedio y la correlación en cada uno de los procesos. Los cálculos completos se encuentran en el apéndice B.

Durante la disminución de la rigidez del potencial tomando en cuenta el protocolo (6.4) y 6.5) calculamos la posición,

$$
\begin{aligned}
x(t)_{1 \rightarrow 2} & =x\left(t_{0}\right)_{1 \rightarrow 2} e^{\frac{\alpha t}{\tau}\left(\frac{2 t \Delta k}{\tau}-(1+\Delta k)\right)}+\frac{\sqrt{\pi} \tau e^{\frac{\alpha(-4 t \Delta k+\tau(1+\Delta k))^{2}}{8 \Delta k \tau^{2}}}}{2 \sqrt{2} \gamma \sqrt{\alpha} \sqrt{\Delta k}} \\
& \left(\operatorname{Erf}\left[\frac{\sqrt{\alpha}(1+\Delta k)}{2 \sqrt{2} \sqrt{\Delta k}}\right]-\operatorname{Erf}\left[\frac{\sqrt{\alpha}(-4 t \Delta k+\tau+\Delta k \tau)}{2 \sqrt{2} \sqrt{\Delta k} \tau}\right]\right) \Gamma(q)
\end{aligned}
$$

El ruido $\Gamma\left(q_{1}\right)$ tiene una distribución Gaussiana, su promedio es cero y su varianza es constante $q_{1}(1+\Delta q)$

El promedio de la posición tiene la siguiente expresión

$$
\left\langle x(t)_{1 \rightarrow 2}\right\rangle=x\left(t_{0}\right)_{1 \rightarrow 2} e^{-\alpha\left[(1+\Delta k) \frac{t}{\tau}-\frac{4 t^{2} \Delta k}{2 \tau^{2}}\right]}
$$

y la correlación cuando $t_{1}=t_{2}$ está dada por

$$
\begin{aligned}
\left\langle x^{2}(t)_{1 \rightarrow 2}\right\rangle & =x\left(t_{0}\right)_{1 \rightarrow 2}^{2} e^{-\frac{\alpha}{\tau}\left[(1+\Delta k)(2 t)-\frac{4 t^{2} \Delta k}{\tau}\right]}+\frac{q_{1}(1+\Delta q) \sqrt{m \pi \gamma \tau}}{\gamma^{2} \sqrt{k_{1} \Delta k}} e^{\frac{\alpha(1+\Delta k)^{2} \tau}{4 \tau \Delta k}} \\
& e^{-\frac{\alpha}{\tau}\left[(1+\Delta k)(2 t)-\frac{4 \Delta k t^{2}}{\tau}\right]}\left(\operatorname{Erf}\left[\frac{\sqrt{\alpha}(1+\Delta k)}{2 \sqrt{\Delta k}}\right]\right. \\
& \left.-\operatorname{Erf}\left[\frac{\sqrt{\alpha}(-4 t \Delta k+\tau+\Delta k \tau)}{2 \sqrt{\Delta k \tau}}\right]\right)
\end{aligned}
$$

En la figura 6.2 vemos la posición de la partícula para una realización, las fluctuaciones están aumentando en el tiempo debido a que la rigidez del potencial disminuye lo cual permite más movimiento, podemos observar su movimiento en comparación con su promedio que decae de manera lenta. 


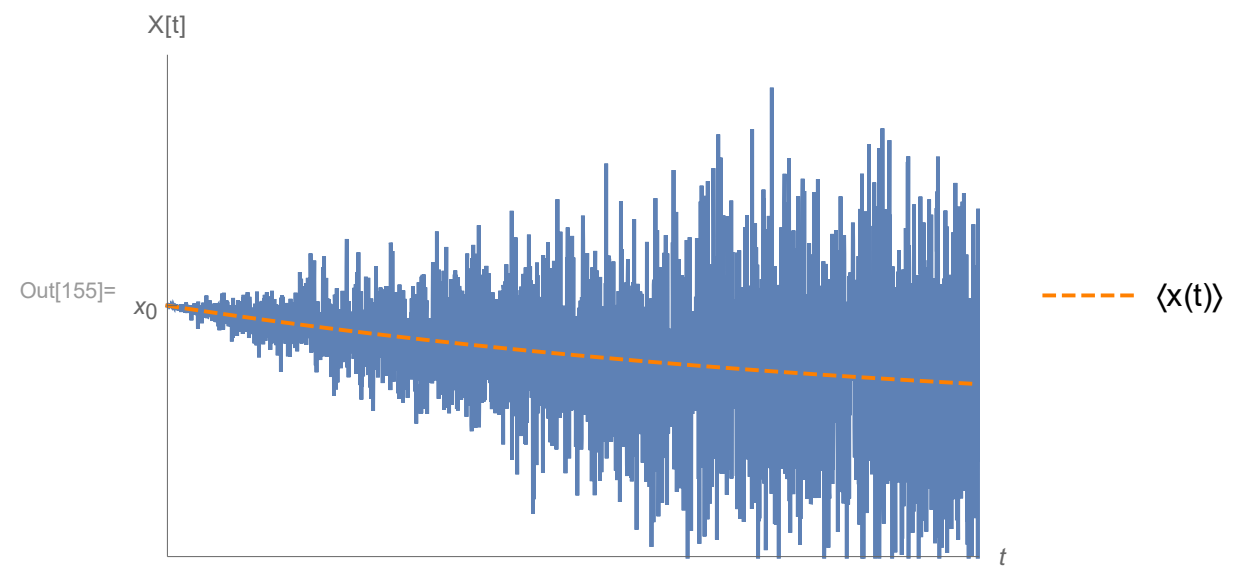

Figura 6.2: La posición de la patícula y su promedio durante la disminución del potencial

En el segundo proceso comenzamos en el tiempo $\frac{\tau}{4}$ la rigidez de la trampa permanece constante (6.6) pero cambia la intensidad del ruido como se indica en (6.7), en este caso la posición está dada por:

$$
x(t)_{2 \rightarrow 3}=x\left(t_{0}\right)_{2 \rightarrow 3} e^{-\alpha\left(\frac{t}{\tau}-\frac{1}{4}\right)}+\frac{1}{\gamma} \int_{\frac{\tau}{4}}^{t} \Gamma\left(t^{\prime}\right) e^{-\frac{\alpha\left(t-t^{\prime}\right)}{\tau}} d t^{\prime}
$$

Debido a su dependencia temporal en la intensidad del ruido, la integral debe de ser evaluada numéricamente. Pero por su propiedad Gaussiana no hay problema en encontrar el promedio de su posición y su correlación.

El promedio está dado por

$$
\left\langle x(t)_{2 \rightarrow 3}\right\rangle=x\left(t_{0}\right)_{2 \rightarrow 3} e^{-\frac{\alpha}{\tau}(t-\tau / 4)}
$$

Y la correlación cuando $t_{1}=t_{2}$

$$
\begin{aligned}
\left\langle x^{2}(t)_{2 \rightarrow 3}\right\rangle & =x\left(t_{0}\right)_{2 \rightarrow 3}^{2} e^{-\frac{\alpha}{\tau}\left(2 t-\frac{\tau}{2}\right)}+\frac{q_{1}}{\gamma^{2}}\left[\left(\frac{2 \Delta q}{\alpha}\left(t+\frac{\tau}{2 \alpha}\right)+\frac{\tau(1+\Delta q)}{2 \alpha}\right)\right. \\
& \left.+e^{-\frac{\alpha}{\tau}\left(2 t-\frac{\tau}{2}\right)}\left(-\frac{2 \Delta q}{\alpha}\left(\frac{\tau}{4}+\frac{\tau}{2 \alpha}\right)-\frac{\tau(1+\Delta q)}{2 \alpha}\right)\right]
\end{aligned}
$$

En la figura 6.3 vemos que la posición de la partícula aunque muestra un aumento en las fluctuaciones se encuentra mucho más limitada en sus movimientos, esto se genera debido al potencial de atrapamiento constante que se está ejerciendo sobre ésta y a la disminución de la intensidad del ruido, vemos que la posición quedan muy cerca del valor promedio.

En el tercer proceso del ciclo el tiempo $t$ solo puede tomar valores entre $2 \tau / 4$ y $3 \tau / 4$ durante este tiempo se realiza un aumento en el confinamiento de la partícula Browniana (6.8) con una intensidad de ruido constante (6.9). 


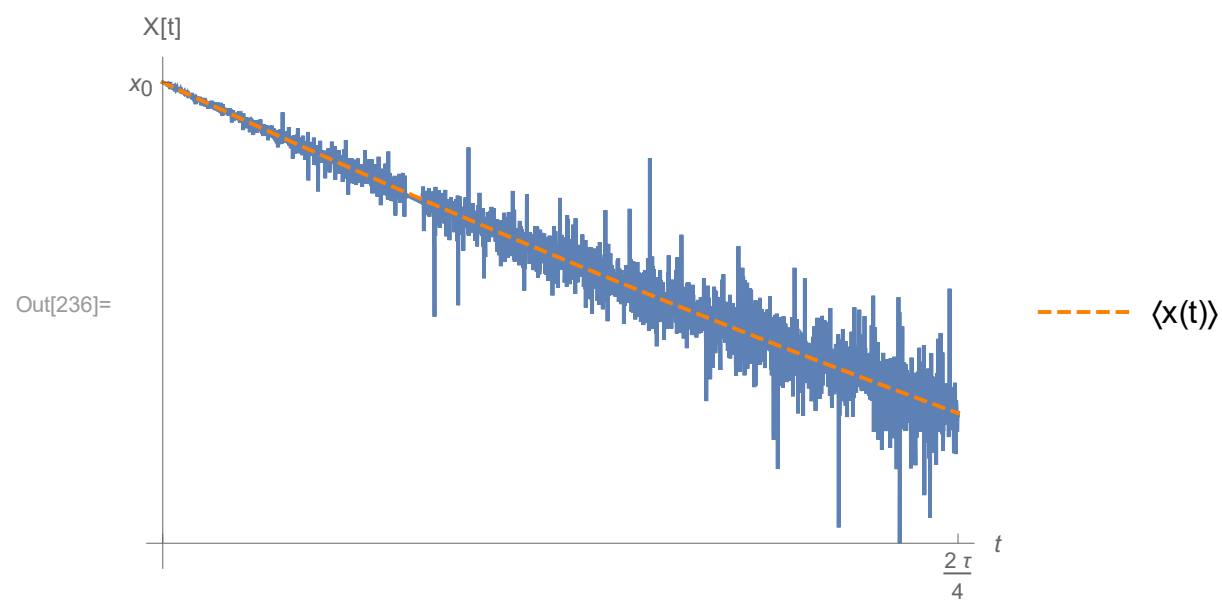

Figura 6.3: Gráfica de la posición durante el segundo proceso del ciclo en el que la intensidad del ruido cambia con una rigidez del potencial constante.

La ecuación que describe la posición de la partícula es

$$
\begin{aligned}
x(t)_{3 \rightarrow 4} & =x\left(t_{0}\right)_{3 \rightarrow 4} e^{-\frac{\alpha(2 t-\tau)(2 t \Delta k+\tau-\Delta k \tau)}{2 \tau^{2}}}-\frac{\sqrt{\pi} \tau e^{-\frac{\alpha(4 t \Delta k+\tau-2 \Delta k \tau)^{2}}{8 \Delta k \tau^{2}}}}{2 \sqrt{2} \gamma \sqrt{\Delta k}} \\
& \left(\operatorname{Erfi}\left[\frac{\sqrt{\alpha}}{2 \sqrt{2} \sqrt{\Delta k}}\right]-\operatorname{Erfi}\left[\frac{\sqrt{\alpha}(4 t \Delta k+\tau-2 \Delta k \tau)}{2 \sqrt{2} \sqrt{\Delta k} \tau}\right]\right) \Gamma\left(q_{1}\right)
\end{aligned}
$$

En donde $\Gamma(t)$ tiene las propiedades del ruido Gaussiano con promedio cero y con intensidad de ruido constante $q_{1}$

En promedio usando las propiedades del ruido, obtenemos

$$
\left\langle x(t)_{3 \rightarrow 4}\right\rangle=x\left(t_{0}\right)_{3 \rightarrow 4} e^{-\frac{\alpha}{\tau}\left(1-2 \Delta k+\frac{4 \Delta k t}{\tau}\right)\left(t-\frac{\tau}{2}\right)}
$$

y la correlación cuando $t_{1}=t_{2}$ está dada por:

$$
\begin{aligned}
& \left\langle x(t)_{3 \rightarrow 4}\right\rangle=x\left(t_{0}\right)_{3 \rightarrow 4}^{2} e^{-\alpha\left(\frac{2 t}{\tau}-\frac{2 \Delta k(2 t)}{\tau}+\frac{\Delta k\left(2 t^{2}\right)}{\tau^{2}}+1-\frac{3 \Delta k}{2}\right)}+\frac{q_{1} e^{-\frac{\alpha}{\tau}\left[(2 t)(1-2 \Delta k)+\frac{2 t^{2}}{\tau}\right]}}{\gamma^{2}} \\
& \frac{q_{1} \tau \sqrt{\pi}}{2 \sqrt{2 \alpha}}\left[\operatorname{Erfi}(\sqrt{2 \alpha}(-1+\Delta k))+\operatorname{Erfi}\left(\frac{\sqrt{\alpha}(2 t+\tau-2 \Delta k \tau)}{\sqrt{2} \tau}\right)\right]
\end{aligned}
$$

El comportamiento de la partícula Browniana en este caso se puede observar en la figura 6.4 .

Con el cuarto proceso realizando el aumento de la intensidad del ruido 6.11 sucede en un tiempo $t$ que va de $3 \tau / 4$ a $\tau$ con una rigidez de potencial constante 6.10 regresamos al estado inicial de los parámetros externo en el ciclo.

La posición de la partícula está dada por la siguiente ecuación

$$
x(t)_{4 \rightarrow 1}=x\left(t_{0}\right)_{4 \rightarrow 1} e^{-\frac{\alpha(1+\Delta k)\left(t-\frac{3 \tau}{4}\right)}{\tau}}+\frac{1}{\gamma} \int_{\frac{3 \tau}{4}}^{t} \Gamma\left(t^{\prime}\right) e^{-\frac{\alpha\left(t-t_{1}\right)(1+\Delta k)}{\tau}} d t^{\prime}
$$




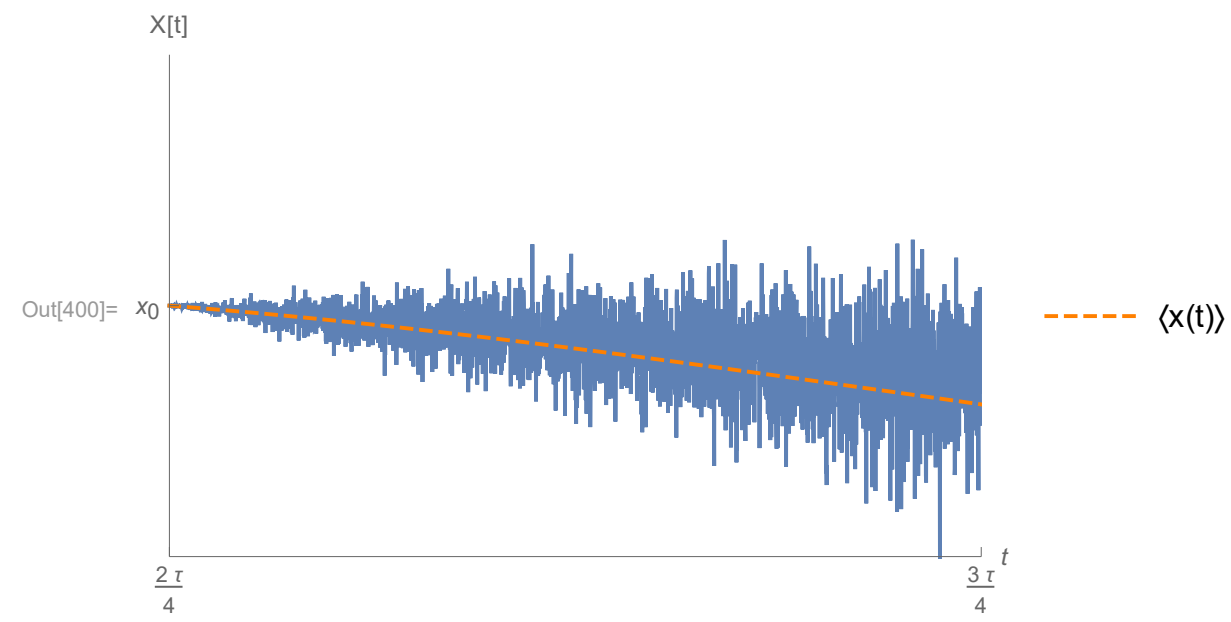

Figura 6.4: Gráfica para el tercer proceso del ciclo, en donde se muestra la posición de la partícula Browniana y su promedio.

Cuya solución por la dependencia temporal del ruido dado por el protocolo 6.11 solo puede realizarse numéricamente. Pero las expresiones del promedio y la correlación pueden ser determinadas gracias a la propiedad Gaussiana del ruido.

El promedio está dado por

$$
\left\langle x(t)_{4 \rightarrow 1}\right\rangle=x\left(t_{0}\right)_{4 \rightarrow 1} e^{-\frac{\alpha(1+\Delta k)}{\tau}\left(t-\frac{3 \tau}{4}\right)}
$$

Y la correlación tiene la siguiente forma cuando $t_{1}=t_{2}$.

$$
\begin{aligned}
&\left\langle x(t)_{4 \rightarrow 1}^{2}\right\rangle=x\left(t_{0}\right)_{4 \rightarrow 1}^{2} e^{-\frac{\alpha(1+\Delta k)}{\tau}\left(2 t-\frac{3 \tau}{2}\right)}+\frac{\tau q_{1}}{2 \gamma^{2} \alpha(1+\Delta k)} \\
& {\left[\left(1-3 \Delta q-\frac{4 \Delta q}{\tau}\left(\frac{\tau}{2 \alpha(1+\Delta k)}\right)\right)+e^{-\frac{\alpha(1+\Delta k)}{\tau}\left(2 t+\frac{3 \tau}{2}\right)}\right.} \\
&\left.\left(-1+3 \Delta q+\frac{4 \Delta q}{\tau}\left(\frac{3 \tau}{4}+\frac{\tau}{2 \alpha(1+\Delta k)}\right)\right)\right]
\end{aligned}
$$

Podemos ver el comportamiento de la partícula durante una realización del proceso en referencia con el promedio en la figura 6.5. Se nota en la imagen que durante este proceso la partícula se encuentra en un potencial de confinamiento más fuerte ya que muestra menos fluctuaciones.

Podríamos realizar el análisis completo termodinámico a partir de esta solución a la ecuación de Langevin, debido a las fluctuaciones que presenta la partícula en cada realización obtendrá una trayectoria diferente y por lo tanto resultados diferentes, en este caso es más conveniente realizar en análisis mediante su distribución de probabilidad dada por la ecuación de Fokker-Planck.

\subsection{Ecuación de Fokker-Planck en el ciclo}

Cuando se hace el análisis con la ecuación de Fokker-Planck nos interesa encontrar la distribución de probabilidad en cada uno de los procesos, de esta manera podremos determinar la probabilidad de que la variable $x$ tenga un valor entre $(x, x+d x)$ al tiempo $t$, la solución se propuso con una forma Gaussiana 


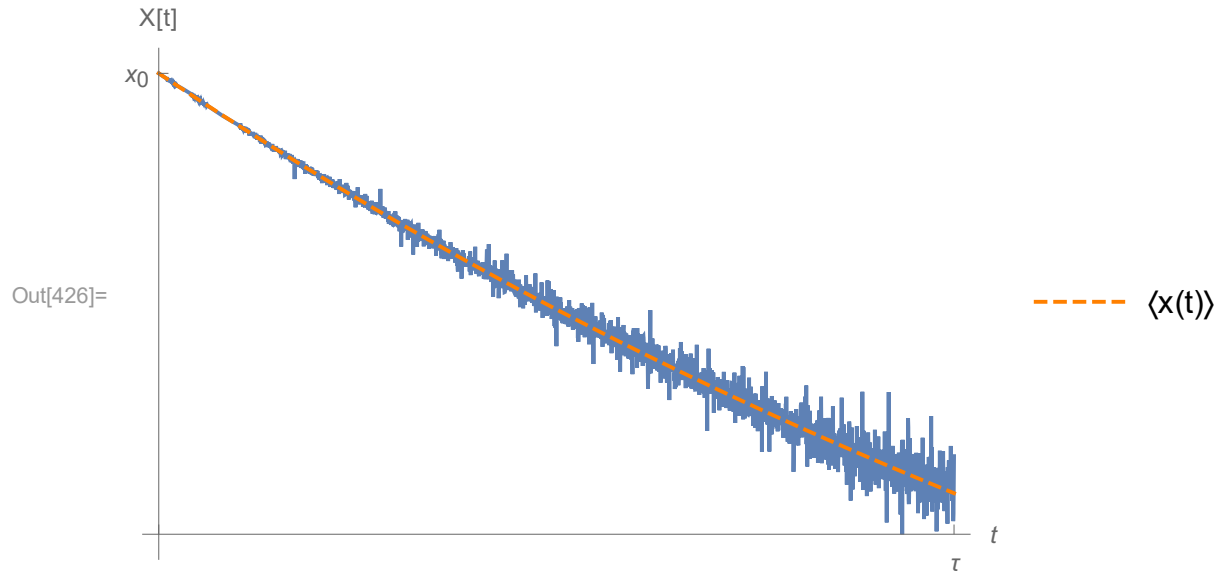

Figura 6.5: Último proceso del ciclo. Gráfica de la posición de una partícula Browniana durante este proceso.

$$
w(x, t)=\sqrt{\frac{\epsilon(t)}{\pi}} e^{-\epsilon(t) x^{2}}
$$

y como vimos en el la sección 4.3 encontrar la distribución de probabilidad se reduce a encontrar $\epsilon(t)$ dada por (4.33),

$$
\frac{1}{\epsilon(t)}=\frac{1}{\epsilon\left(t_{0}\right)} e^{-\frac{2}{\gamma m} \int_{t_{0}}^{t} k\left(t^{\prime}\right) d t^{\prime}}+\frac{2}{\gamma^{2}} \int_{t_{0}}^{t} q\left(t^{\prime}\right) e^{-\frac{2}{\gamma m} \int_{t^{\prime}}^{t} k\left(t^{\prime \prime}\right) d t^{\prime \prime}} d t^{\prime}
$$

Con la aplicación del protocolo propuesto solo resta resolver las integrales en el tiempo para encontrar la solución, además se debe de tomar en cuenta la condición inicial de la partícula. En el caso del ciclo, solo durante el primer proceso la condición inicial de la partícula Browniana corresponde al equilibrio térmico con el baño en el que se encuentra, de esta manera $\epsilon\left(t_{0}\right)$ es

$$
\epsilon\left(t_{0}\right)=\frac{\gamma k\left(t_{0}\right)}{m q\left(t_{0}\right)}
$$

Los pasos a detalle de los siguientes cálculos se encuentran en el Apéndice C

En el primer proceso $k(t)$ y $q(t)$ están dados por (6.4) y (6.5) respectivamente. El protocolo de este proceso implica que el confinamiento de la partícula Browniana disminuye. Tomando en cuenta que inicialmente la partícula se encuentra en su estado de equilibrio con el medio, y después al comenzar el proceso la sacamos de su equilibrio, podemos escribir $\epsilon(t)$ de forma adimensional de la siguiente manera.

$$
\begin{array}{r}
\frac{\epsilon(0)_{1 \rightarrow 2}}{\epsilon_{1 \rightarrow 2}(t)}=e^{\frac{2 \alpha t}{\tau}\left[2 \Delta k \frac{t}{\tau}-(\Delta k+1)\right]}+\frac{\sqrt{\alpha}(1+\Delta k) \sqrt{\pi} e^{\frac{\alpha}{4 \Delta k}\left(-4 \Delta k \frac{t}{\tau}+(\Delta k+1)\right.}}{2 \sqrt{\Delta k}} \\
\left(\operatorname{Erf}\left[\frac{\sqrt{\alpha}(\Delta k+1)}{2 \sqrt{\Delta k}}\right]-\operatorname{Erf}\left[\frac{\sqrt{\alpha}\left(-4 \Delta k \frac{t}{\tau}+(\Delta k+1)\right)}{2 \sqrt{\Delta k}}\right]\right)
\end{array}
$$


Una vez que se le asignan valores a $\Delta q$ y $\Delta k$ los resultados tienen dependencia con $\alpha$ y $t / \tau$ y esto sucede en cada uno de los procesos.

En este caso, el proceso está limitado por los valores que puede tomar el tiempo, ya que el protocolo se realiza para un tiempo $t$ finito que va de 0 a $\frac{\tau}{4}$. En la solución 6.30 podemos ver que la dependencia temporal está dada por la fracción adimensional $\frac{t}{\tau}$ a la que llamaremos $t_{a d}$ de esta manera el primer proceso es válido para

$$
0 \leq t_{a d} \leq \frac{1}{4}
$$

Con $t_{a d}=0$ la solución se reduce al valor de la condición inicial y en $t_{a d}=\frac{1}{4}$ nos indica su valor al final del proceso, si el proceso fuera ideal y se realizara cuasiestáticamente entonces $\frac{1}{\epsilon(t)}$ final coincidiría con su valor en equilibrio térmico dado por $\frac{1}{\epsilon\left(t=\frac{\tau}{4}\right)}=\frac{m q_{2}}{\gamma k_{1}}$. Realizando la resta de ambas expresiones encontramos la raíz numérica para encontrar en qué $\alpha$ el valor de $\frac{1}{\epsilon\left(t=\frac{\tau}{4}\right)}$ se aproxima a su valor de equilibrio. $\mathrm{Al}$ hacer esto encontramos que para $\alpha \approx 127$ el sistema podría estar en un estado de equilibrio, recordemos que $\alpha$ (6.1) mide el cociente de los tiempos característicos en el sistema, esto implica que un $\alpha$ grande puede relacionarse con un tiempo de proceso grande comparado con el tiempo de relajación del sistema lo que nos llevaría al caso cuasiestático o por ejemplo que la rigidez inicial $k_{1}$ es muy grande comparada con $\frac{\tau}{m \gamma}, \alpha$ grande se puede interpretar de diversas maneras y los datos experimentales nos pueden ayudar a determinar en qué situaciones se está evaluando el proceso.

Durante el segundo proceso del ciclo la disminución del ruido 6.7 a un potencial constante 6.6 nos lleva al siguiente resultado, en este caso no necesariamente la condición inicial del sistema $\epsilon(t)$ corresponde al estado de equilibrio $\frac{1}{\epsilon\left(\frac{\tau}{4}\right)_{e q}}=\frac{m q_{2}}{\gamma k_{1}}$

$$
\begin{aligned}
& \frac{\epsilon(\tau / 4)_{1 \rightarrow 2}}{\epsilon_{2 \rightarrow 3}(t)}=e^{-2 \alpha\left(\frac{t}{\tau}-\frac{1}{4}\right)}-\frac{\epsilon(\tau / 4)_{1 \rightarrow 2}}{\epsilon_{e q} \alpha(1+\Delta q)} \\
& \left(4 \frac{t}{\tau} \alpha \Delta q+\left(2 \Delta q\left[-1+e^{-\frac{2 \alpha}{\tau}(t-\tau / 4)}\right]+\alpha\left[-1-2 \Delta q+e^{-2 \alpha\left(\frac{t}{\tau}-\frac{1}{4}\right)}(1+\Delta q)\right]\right)\right)
\end{aligned}
$$

en el caso en el que $\frac{\epsilon(\tau / 4)_{1 \rightarrow 2}}{\epsilon_{e q}}=1$ se cumpla entonces llegamos a

$$
\begin{aligned}
\frac{\epsilon\left(t_{0}\right)_{2 \rightarrow 3}}{\epsilon_{2 \rightarrow 3}(t)} & =e^{-2 \alpha\left(\frac{t}{\tau}-\frac{1}{4}\right)}-\frac{4 t \alpha \Delta q}{\alpha \tau(1+\Delta q)} \\
& -\frac{2 \Delta q\left[-1+e^{-2 \alpha\left(\frac{t}{\tau}-\frac{1}{4}\right)}\right]+\alpha\left[-1-2 \Delta q+e^{-2 \alpha\left(\frac{t}{\tau}-\frac{1}{4}\right)}(1+\Delta q)\right]}{\alpha(1+\Delta q)}
\end{aligned}
$$

La soluciones están expresadas en términos de la fracción adimensional $t_{a d}=\frac{t}{\tau} \mathrm{y}$ ya que el tiempo se encuentra limitado, la fracción adimensional solo puede ir de

$$
\frac{1}{4} \leq t_{a d} \leq \frac{1}{2}
$$


evaluada en $t_{a d}=\frac{1}{4}$ la solución nos indica que $\epsilon$ se encuentra en el $\epsilon$ inicial, cuando se evalúa en el tiempo final entonces encontramos el valor de $\epsilon$ fuera de equilibrio, que si el proceso se realiza en caso cuasiestático podríamos regresar al caso de equilibrio.

El tercer proceso es un aumento en la rigidez del potencial con una intensidad de ruido constante. Durante este proceso la solución es la siguiente,

$$
\begin{gathered}
\frac{\epsilon(2 \tau / 4)_{2 \rightarrow 3}}{\epsilon_{3 \rightarrow 4}(t)}=e^{-\alpha\left(2 \frac{t}{\tau}-1\right)\left(2 \Delta k \frac{t}{\tau}+1-\Delta k\right)}-\frac{\epsilon(2 \tau / 4)_{2 \rightarrow 3}}{\epsilon_{e q}} \frac{\sqrt{\alpha} \sqrt{\pi} e^{-\frac{\alpha}{4 \Delta k}\left(4 \Delta k \frac{t}{\tau}-2 \Delta k+1\right)^{2}}}{2 \sqrt{\Delta k}} \\
{\left[\operatorname{Erfi}\left(\frac{\sqrt{\alpha}}{2 \sqrt{\Delta k}}\right)-\operatorname{Erfi}\left(\frac{\sqrt{\alpha}\left(4 \Delta k \frac{t}{\tau}+1-2 \Delta k\right)}{2 \sqrt{\Delta k}}\right)\right]}
\end{gathered}
$$

En el caso en el que la condición inicial coincide con el estado de equilibrio

$$
\begin{gathered}
\frac{\epsilon\left(t_{0}\right)_{3 \rightarrow 4}}{\epsilon_{3 \rightarrow 4}(t)}=e^{-\alpha\left(2 \frac{t}{\tau}-1\right)\left(2 \Delta k \frac{t}{\tau}+1-\Delta k\right)}-\frac{\sqrt{\alpha} \sqrt{\pi} e^{-\frac{\alpha}{4 \Delta k}\left(4 \Delta k \frac{t}{\tau}-2 \Delta k+1\right)^{2}}}{2 \sqrt{\Delta k}} \\
{\left[\operatorname{Erfi}\left(\frac{\sqrt{\alpha}}{2 \sqrt{\Delta k}}\right)-\operatorname{Erfi}\left(\frac{\sqrt{\alpha}\left(4 \Delta k \frac{t}{\tau}+1-2 \Delta k\right)}{2 \sqrt{\Delta k}}\right)\right]}
\end{gathered}
$$

Ambas soluciones están en función de la fracción adimensional $t_{a d}$, durante este proceso la solución está limitada para

$$
\frac{1}{2} \leq t_{a d} \leq \frac{3}{4}
$$

cuando la analizamos en el tiempo inicial del proceso la solución es la $\epsilon(t)$, cuando lo analizamos en el tiempo final obtenemos $\epsilon(t)$ fuera de equilibrio, para regresar que el proceso pueda tomarse en cuenta como un proceso en equilibrio entonces, $\alpha$, tiene que ser muy grande.

El último proceso que cierra el ciclo es un aumento en la intensidad del ruido mientras se mantiene la rigidez del potencial de atrapamiento constante. La solución la encontraremos resolviendo tres integrales

$$
\begin{aligned}
& \frac{\epsilon(3 \tau / 4)_{3 \rightarrow 4}}{\epsilon_{4 \rightarrow 1}(t)}=e^{2 \alpha(1+\Delta k)\left(-\frac{t}{\tau}+\frac{3}{4}\right)}+\frac{\epsilon(3 \tau / 4)_{3 \rightarrow 4}}{\epsilon_{e q} \alpha(1+\Delta k)}\left[4 \alpha \Delta q \frac{t}{\tau}(1+\Delta k)+2 \Delta q\right. \\
& \left.\left(-1+e^{2 \alpha(1+\Delta k)\left(-\frac{t}{\tau}+\frac{3}{4}\right)}\right)-\alpha(1+\Delta k)\left(-1+e^{2 \alpha(1+\Delta k)\left(-\frac{t}{\tau}+\frac{3}{4}\right)}+3 \Delta q\right)\right]
\end{aligned}
$$

Cuando la $\epsilon_{e q}$ coincide con el valor de la condición inicial entonces tenemos

$$
\begin{aligned}
& \frac{\epsilon\left(t_{0}\right)_{4 \rightarrow 1}}{\epsilon_{4 \rightarrow 1}(t)}=e^{2 \alpha(1+\Delta k)\left(-\frac{t}{\tau}+\frac{3}{4}\right)}+\frac{1}{\alpha(1+\Delta k)}\left[4 \alpha \Delta q \frac{t}{\tau}(1+\Delta k)+2 \Delta q\right. \\
& \left.\left(-1+e^{2 \alpha(1+\Delta k)\left(-\frac{t}{\tau}+\frac{3}{4}\right)}\right)-\alpha(1+\Delta k)\left(-1+e^{2 \alpha(1+\Delta k)\left(-\frac{t}{\tau}+\frac{3}{4}\right)}+3 \Delta q\right)\right]
\end{aligned}
$$

Las soluciones están en función de la fracción adimensional de los tiempos que llamamos $t_{a d}$, este tiempo va de

$$
\frac{3}{4} \leq t_{a d} \leq 1
$$


La solución regresa al valor esperado en el tiempo inicial y en el tiempo final $\epsilon(t)_{4 \rightarrow 1}$ el sistema llega al estado de equilibrio cuando $\alpha$ es muy grande.

En cada uno de los procesos se puede llegar al estado de equilibrio con el medio en el que se encuentra la partícula siempre y cuando $\alpha$ sea muy grande. Esto implica que el proceso se tiene que realizar de manera cuasiestática o durante un tiempo $\tau$ infinito para que la partícula Browniana pueda realizar un ciclo de Stirling reversible. Claramente las fluctuaciones que siente la partícula y la dependencia temporal en el protocolo sacan del equilibrio a nuestro sistema y solo en un caso muy ideal se podría tomar en cuenta un cuasi equilibrio.

Hasta aquí solo hemos determinado $\epsilon$, con estas expresiones podemos encontrar la distribución de probabilidad, y con la distribución de probabilidad podemos determinar los promedios sobre el ensamble de muchas realizaciones. La termodinámica del sistema, que como ya vimos sólo en casos muy específicos se encuentra en equilibrio, la determinaremos con ayuda de las expresiones que acabamos de encontrar. 



\section{Capítulo 7}

\section{Termodinámica del ciclo}

Para un sistema que es manipulado por pinzas ópticas, el potencial del sistema se puede modelar como un potencial del tipo oscilador armónico en una dimensión. Hacemos uso de esta aproximación para encontrar las ecuaciones que definirán la termodinámica de la partícula de estudio, se debe de tener especial cuidado en mantener la dependencia temporal en la rigidez de la trampa $k(t)$ y en la intensidad del ruido $q(t)$ y recordar que se trata del caso sobre-amortiguado. En la primera parte encontraremos la expresión del calor, posteriormente desarrollaremos la expresión para el trabajo y la entropía. Finalmente analizaremos la termodinámica de cada proceso del ciclo de Stirling dado por el protocolo.

\subsubsection{Calor}

El intercambio de energía que se realiza durante un proceso entre el sistema y los alrededores nos indica el cambio del calor (3.14) del sistema.

$$
d Q=\frac{\partial U(x, t)}{\partial x} \cdot d x(t)
$$

en nuestro modelo el potencial generado por las pinzas ópticas es un potencial armónico en una dimensión, sustituyendo el valor y dejando la dependencia temporal del parámetro externo, que en este caso es la rigidez de la trampa $k(t)$ llegamos a la siguiente expresión.

$$
\begin{aligned}
d Q & =k(t) x(t) d x \\
& =\frac{k(t)}{2} d x^{2}
\end{aligned}
$$

Integrando podemos determinar el cambio de calor que sucede durante el proceso

$$
\Delta Q=\int_{x\left(t_{0}\right)}^{x(t)} \frac{k(t)}{2} d\left(x^{2}\right)
$$

siguiente

Por las propiedades aleatorias de los sistemas pequeños el calor tendrá un valor diferente en cada realización del proceso, aunque se lleve a cabo mediante el 
mismo protocolo. Nos interesa encontrar el promedio de una gran cantidad de realizaciones del proceso. El promedio no actúa sobre la rigidez del resorte debido a que es modificada externamente de manera determinista.

$$
\langle\Delta Q\rangle=\int_{x(t 0)}^{x(t)} \frac{k(t)}{2} d\left\langle x^{2}\right\rangle
$$

El promedio lo encontramos utilizando la distribución de probabilidad que es solución a la ecuación de Fokker-Planck (4.31). Usando esta cantidad y tomando en cuenta que la distribución debe de cumplir con 4.33 podemos encontrar el promedio de $x^{2}$.

$$
\begin{aligned}
\left\langle x^{2}\right\rangle & =\int w(x, t) x^{2} d x \\
& =\frac{1}{2 \epsilon(t)}
\end{aligned}
$$

haciendo uso de esta relación y la relación 4.32 encontramos la expresión del calor

$$
\langle\Delta Q\rangle=-\int_{t 0}^{t}\left(\frac{k(t)^{2}}{2 m \gamma \epsilon(t)}-\frac{k(t) q(t)}{2 \gamma^{2}}\right) d t
$$

Esta es la ecuación que describe el cambio promedio del calor durante un proceso que es realizado mediante modificaciones en los parámetros externos, podemos observar que esta expresión depende completamente de éstos, se puede modificar la rigidez de la trampa y dejar constante la intensidad del ruido, se puede hacer de la manera contraria, o bien variar las ambas al mismo tiempo, todo depende del proceso que se quiere estudiar.

\subsubsection{Trabajo}

Para encontrar el trabajo seguimos el mismo proceso, aplicamos el potencial tipo oscilador armónico en la definición de trabajo

$$
\begin{aligned}
d W(x, t) & =\frac{\partial\left(\frac{1}{2} k(t) x(t)^{2}\right)}{\partial k(t)} d k(t) \\
& =\left(\frac{1}{2} x(t)^{2}\right) \frac{d k(t)}{d t} d t
\end{aligned}
$$

Integramos para determinar el cambio de trabajo que sucede en cada proceso.

$$
\Delta W(x, t)=\int_{t_{0}}^{t_{f}}\left(\frac{1}{2} x(t)^{2}\right) \frac{d k(t)}{d t} d t
$$

Al ser la posición una variable aleatoria, entonces el trabajo es estocástico y tendrá al igual que el calor, un valor diferente en cada realización del proceso. El promedio está dado por 


$$
\langle\Delta W(x, t)\rangle=\int_{t_{0}}^{t_{f}} \frac{1}{2}\left\langle x(t)^{2}\right\rangle \frac{d k(t)}{d t} d t
$$

Usando la distribución de probabilidad (4.31) determinamos que el promedio de $x^{2}$ es.

$$
\left\langle x(t)^{2}\right\rangle=\frac{1}{2} \frac{1}{\epsilon(t)}
$$

Entonces la expresión final del promedio del trabajo es

$$
\langle\Delta W(x, t)\rangle=\int_{t_{0}}^{t_{f}} \frac{1}{4 \epsilon(t)} \frac{d k(t)}{d t} d t
$$

y siempre será cero cuando no exista cambio en la rigidez $k(t)$ del potencial. Las implicaciones de esto es otro paralelismo con la termodinámica clásica para un gas ideal, ya que la rigidez de la trampa es proporcional a mantener un volumen en el sistema constante. Como bien sabemos durante cualquier proceso isocórico no se realiza trabajo.

\subsubsection{Entropía}

La entropía de Gibbs para sistemas fuera de equilibrio nos indica que existe una entropía que depende de la trayectoria de la partícula, podemos escribirla como

$$
S^{*}(x, t)=\frac{s}{k_{B}}=-\operatorname{Ln} w(x, t)
$$

Para determinar la entropía sobre una trayectoria reescribirmos el logaritmo de $w$ usando las propiedades de los logaritmos

$$
\operatorname{Ln}[w(x(t), t)]=\frac{\operatorname{Ln}\left[\frac{\epsilon(t)}{\pi}\right]}{2}-\epsilon(t) x^{2}
$$

de esta manera la entropía está dada por

$$
s(t)=-\frac{\operatorname{Ln}\left[\frac{\epsilon(t)}{\pi}\right]}{2}+\epsilon(t) x^{2}
$$

en donde $x(t)$ está determinada por el proceso estocástico por lo que un resultado con senntido físico está dado por el promedio sobre muchas realizaciones

$$
\langle s(t)\rangle=-\frac{1}{2} \operatorname{Ln}\left[\frac{\epsilon(t)}{\pi}\right]+\epsilon(t)\left\langle x^{2}\right\rangle
$$

Pero el promedio de $x^{2}$ como hemos visto está dado por 7.5 , por lo tanto

$$
\langle s(t)\rangle=-\frac{1}{2} \operatorname{Ln}\left[\frac{\epsilon(t)}{\pi}\right]+\frac{1}{2}
$$

El cambio de entropía que se genera durante el proceso está dado por

$$
\Delta\langle s(t)\rangle=\left\langle s\left(t_{f}\right)\right\rangle-\left\langle s\left(t_{o}\right)\right\rangle
$$




$$
=\frac{1}{2}\left(\operatorname{Ln}\left[\frac{\epsilon\left(t_{o}\right)}{\epsilon\left(t_{f}\right)}\right]\right)
$$

El cambio de entropía durante cada proceso del ciclo depende del logaritmo de la razón entre las $\epsilon(t)$.

Todas las cantidades termodinámicas que hemos encontrado dependen del protocolo aquí planteado para realizar el ciclo de Stirling. Comenzaremos con el análisis de estas cantidades en los cuatro procesos del ciclo, tomaremos en cuenta como condición inicial de cada uno de los procesos que el sistema se encuentra en un equilibrio con el medio y por lo tanto

$$
\epsilon\left(t_{0}\right)=\epsilon_{e q}=\frac{\gamma k\left(t_{0}\right)}{m q\left(t_{0}\right)}
$$

Los cálculos a detalle están realizados en los Apéndices D, E y F.

\subsection{Disminución del potencial}

El primer proceso es el cambio en la rigidez del potencial 6.5 mientras se mantiene constante el ruido 6.4). La forma de $\epsilon(t)$ está dada por (6.30), con $\epsilon\left(t_{0}\right)=\frac{\gamma k_{2}}{m q_{2}}$ que se puede reescribir en términos de las variables adimensionales como $\epsilon\left(t_{0}\right)=\frac{\gamma k_{1}(\Delta k+1)}{m q_{1}(\Delta q+1)}$ de esta manera definimos una constante $M=\frac{\gamma k_{1}}{m q_{1}}$ y podemos reescribir $\epsilon\left(t_{0}\right)=\frac{M(\Delta k+1)}{(\Delta q+1)}$.

Las unidades de la expresión del calor quedan expresadas en la fracción $\frac{k_{1}}{M}$ que tiene unidades de energía, escribiendo $\frac{M\langle\Delta Q\rangle}{k_{1}}=\langle\Delta Q\rangle^{*}$ llegamos a una expresión adimensional para el calor

$$
\begin{aligned}
& \left\langle\Delta Q_{1 \rightarrow 2}\right\rangle^{*}=\frac{\alpha(1+\Delta q)}{2(1+\Delta k)}\left[\frac { 2 \sqrt { \Delta k } } { \alpha ^ { 3 / 2 } } e ^ { - \frac { \alpha ( \Delta k + 1 ) ^ { 2 } } { 4 \Delta k } } \left(\left(\frac{\sqrt{\alpha} e^{\left(\frac{\sqrt{\alpha}}{2 \sqrt{\Delta k}}\right)^{2}}}{4 \sqrt{\Delta k}}-\frac{1}{4} \sqrt{\pi} \operatorname{Erfi}\left[\frac{\sqrt{\alpha}}{2 \sqrt{\Delta k}}\right]\right)\right.\right. \\
& \left.-\left(\frac{\sqrt{\alpha}(\Delta k+1) e^{\left(\frac{\sqrt{\alpha}}{2 \sqrt{\Delta k}}(\Delta k+1)\right)^{2}}}{4 \sqrt{\Delta k}}-\frac{1}{4} \sqrt{\pi} \operatorname{Erfi}\left[\frac{\sqrt{\alpha}}{2 \sqrt{\Delta k}}(\Delta k+1)\right]\right)\right) \\
& +\frac{(1+\Delta k) \sqrt{\pi}}{\alpha} \operatorname{Erf}\left[\frac{\sqrt{\alpha}(\Delta k+1)}{2 \sqrt{\Delta k}}\right]\left(\left(\frac{\sqrt{\alpha} e^{\left(\frac{\sqrt{\alpha}}{2 \sqrt{\Delta k}}\right)^{2}}}{4 \sqrt{\Delta k}}-\frac{1}{4} \sqrt{\pi} \operatorname{Erfi}\left[\frac{\sqrt{\alpha}}{2 \sqrt{\Delta k}}\right]\right)\right. \\
& \left.-\left(\frac{\sqrt{\alpha}(\Delta k+1) e^{\left(\frac{\sqrt{\alpha}}{2 \sqrt{\Delta k}}(\Delta k+1)\right)^{2}}}{4 \sqrt{\Delta k}}-\frac{1}{4} \sqrt{\pi} \operatorname{Erfi}\left[\frac{\sqrt{\alpha}}{2 \sqrt{\Delta k}}(\Delta k+1)\right]\right)\right) \\
& -\frac{(1+\Delta k) \sqrt{\pi}}{\alpha}\left(\frac{\left(\frac{\sqrt{\alpha}}{2 \sqrt{\Delta k}}\right)^{2}\left(-1+F\left[(1,1),\left(\frac{1}{2}, 2\right),\left(\frac{\sqrt{\alpha}}{2 \sqrt{\Delta k}}\right)^{2}\right]\right)}{2 \sqrt{\pi}}\right.
\end{aligned}
$$




$$
\begin{aligned}
& \left.-\frac{\left(\frac{\sqrt{\alpha}}{2 \sqrt{\Delta k}}(\Delta k+1)\right)^{2}\left(-1+F\left[(1,1),\left(\frac{1}{2}, 2\right),\left(\frac{\sqrt{\alpha}}{2 \sqrt{\Delta k}}(\Delta k+1)\right)^{2}\right]\right)}{2 \sqrt{\pi}}\right) \\
& \left.+\frac{(1+\Delta k)(2+\Delta k)}{8}\right]
\end{aligned}
$$

Para analizar el resultado damos valores numéricos a las variables adimensionales. $\Delta k=1$ y $\Delta q=1$ tomando esto en cuenta la solución queda en términos de $\alpha$, en el límite cuando $\alpha$ tiende a infinito el cambio en el calor tiende al valor

$$
\lim _{\alpha \rightarrow \infty}\left\langle\Delta Q_{1 \rightarrow 2}\right\rangle^{*}=\frac{\operatorname{Ln}(2)}{2}
$$

Al graficar el promedio del calor encontramos el comportamiento mostrado en la figura 7.1 El cambio de calor positivo indica que el sistema está absorbiendo calor

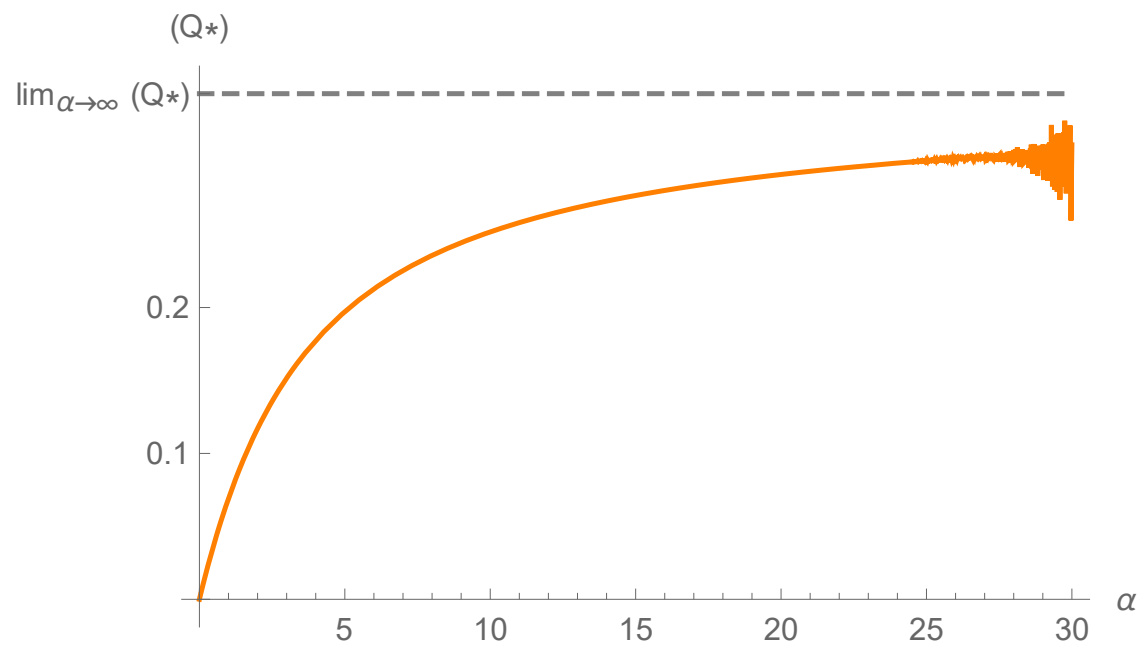

Figura 7.1: Gráfica del calor adimensional durante el primer proceso en función de $\alpha$ para $\Delta k=1$ y $\Delta q=1$

de sus alrededores, este cambio tiende a su valor límite mientras $\alpha$ es más grande pero solo cuando es infinito alcanza el valor del límite.

Para encontrar el promedio del trabajo realizado es necesario encontrar el cambio en la rigidez del potencial

$$
\dot{k}(t)=\frac{d k(t)}{d t}=\frac{k_{1}-k_{2}}{\tau} 4
$$

Tomamos en cuenta que durante este proceso la condición inicial está dada por

$$
\epsilon\left(t_{0}\right)=\frac{\gamma k_{2}}{m q_{2}}=\frac{\gamma k_{1}(1+\Delta k)}{m q_{1}(\Delta q)}=\frac{M(1+\Delta k)}{(\Delta q)}
$$

de esta manera podemos reescribir el cambio en la rigidez del potencial como

$$
\frac{k_{1}-k_{2}}{\epsilon\left(t_{0}\right) \tau}=-\frac{k_{1} \Delta k(1+\Delta q)}{\tau M(1+\Delta k)}
$$


Las unidades del trabajo en el sistema internacional son $[W]=J=\frac{K g m^{2}}{s^{2}}$ la expresion del calor queda en términos de las fracción de $k_{1}=\frac{K g}{s^{2}}$ y $\varepsilon=\frac{1}{m^{2}}$, que forma una fracción con unidades de trabajo $\frac{k_{1}}{\varepsilon}=\frac{K g m^{2}}{s^{2}}$, por lo tanto podemos escribir una expresión adimensional del trabajo $\langle W\rangle^{*}=\frac{\varepsilon\langle W\rangle}{k_{1}}$, de esta manera

$$
\begin{aligned}
& \left\langle\Delta W_{1 \rightarrow 2}\right\rangle^{*}=-\frac{\sqrt{\pi} \sqrt{\Delta k}(1+\Delta q) e^{-\frac{\alpha(\Delta k+1)^{2}}{4 \Delta k}}}{4 \sqrt{\alpha}(1+\Delta k)}\left\{-\operatorname{Erfi}\left[\frac{\sqrt{\alpha}}{2 \sqrt{\Delta k}}\right]\right. \\
& +\operatorname{Erfi}\left[\frac{\sqrt{\alpha}(1+\Delta k)}{2 \sqrt{\Delta k}}\right]+\frac{\sqrt{\alpha}(1+\Delta k) e^{\frac{\alpha(\Delta k+1)^{2}}{4 \Delta k}}}{4 \sqrt{\pi} \Delta k^{3 / 2}}\left(2 \pi \Delta k \operatorname{Erf}\left[\frac{\alpha(\Delta k+1)}{2 \sqrt{\Delta k}}\right]\right. \\
& \left.\left(\operatorname{Erfi}\left[\frac{\sqrt{\alpha}(1+\Delta k)}{2 \sqrt{\Delta k}}\right]-\operatorname{Erfi}\left[\frac{\sqrt{\alpha}}{2 \sqrt{\Delta k}}\right]\right)\right)+\alpha_{2} F_{2}\left[(1,1),\left(\frac{3}{2}, 2\right), \frac{\alpha}{4 \Delta k}\right] \\
& \left.-\alpha(\Delta k+1)^{2}{ }_{2} F_{2}\left[(1,1),\left(\frac{3}{2}, 2\right), \frac{\alpha(1+\Delta k)^{2}}{4 \Delta k}\right]\right\}
\end{aligned}
$$

Este trabajo solo depende del cambio de la rigidez del potencial $\Delta k$, del cambio en la intensidad del ruido $\Delta q$ y de la variable adimensional $\alpha=\frac{k_{1} \tau}{m \gamma}$.

Damos valores a las variables adimensionales $\Delta k=1$ y $\Delta q=1$ para graficar el trabajo con respecto a $\alpha$. Lo primero que nos interesa encontrar es el límite cuando $\alpha$ tiende a infinito

$$
\lim _{\alpha \rightarrow \infty}\left\langle\Delta W_{1 \rightarrow 2}\right\rangle^{*}=-\frac{\operatorname{Ln}[2]}{2}
$$

El límite en valor absoluto es igual al calor, al graficar encontramos el siguiente comportamiento

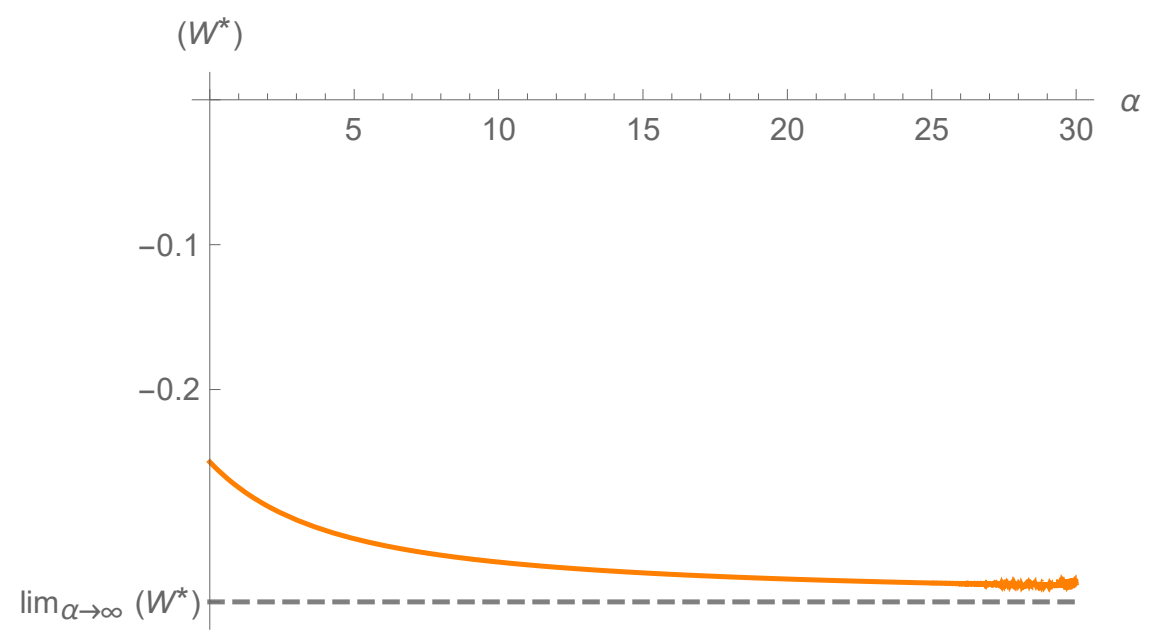

Figura 7.2: Gráfica del trabajo adimensional durante el primer proceso en función de $\alpha$ para $\Delta k=1$ y $\Delta q=1$

El promedio del trabajo siempre es negativo lo cual indica que la partícula Browniana hace trabajo sobre sus alrededores y tiende de manera rápida al valor del límite, pero solo llega a este cuando $\alpha$ es infinito, este caso nos puede indicar el valor del trabajo que se obtiene cuando se toma al sistema en semi equilibrio. El 
que en este límite el trabajo ejercido sobre los alrededores sea igual que el calor que está recibiendo la partícula nos lleva al cumplimiento de la primera ley de la termodinámica para un gas ideal.

$$
\Delta W+\Delta Q=0
$$

Es decir que la energía "interna "depende únicamente de la intensidad del ruido.

Para encontrar la entropía a partir de la ecuación (7.12 tomamos en cuenta que el proceso de 1 a 2 se lleva a cabo en un tiempo de $t_{0}=0$ a $t_{f}=\frac{\tau}{4}$ debemos de evaluar la solución en el tiempo inicial y en el tiempo final. En el tiempo inicial tiene el valor de la condición inicial, como ya se había mencionado antes y cuando evaluamos en el tiempo final

$$
\begin{aligned}
& \frac{1}{\epsilon_{1 \rightarrow 2}\left(\frac{\tau}{4}\right)}=\frac{1}{\epsilon\left(t_{0}\right)_{1 \rightarrow 2}}\left(e^{\frac{\alpha}{2}\left[\Delta k \frac{1}{2}-(\Delta k+1)\right]}+\frac{\sqrt{\alpha}(1+\Delta k) \sqrt{\pi} e^{\frac{\alpha}{4 \Delta k}}}{2 \sqrt{\Delta k}}\right. \\
& \left.\left(\operatorname{Erf}\left[\frac{\sqrt{\alpha}(\Delta k+1)}{2 \sqrt{\Delta k}}\right]-\operatorname{Erf}\left[\frac{\sqrt{\alpha}}{2 \sqrt{\Delta k}}\right]\right)\right)
\end{aligned}
$$

$\mathrm{Al}$ hacer los cálculos para encontrar el promedio de la entropía encontramos

$$
\begin{aligned}
\Delta\langle s\rangle_{1 \rightarrow 2} & =\frac{1}{2} \operatorname{Ln}\left[\frac{\epsilon\left(t_{0}\right)_{1 \rightarrow 2}}{\epsilon\left(t_{f}\right)_{1 \rightarrow 2}}\right] \\
& =\frac{1}{2} \operatorname{Ln}\left[e^{\frac{\alpha}{2}\left[\Delta k \frac{1}{2}-(\Delta k+1)\right]}+\frac{\sqrt{\alpha}(1+\Delta k) \sqrt{\pi} e^{\frac{\alpha}{4 \Delta k}}}{2 \sqrt{\Delta k}}\right. \\
& \left.\left(\operatorname{Erf}\left[\frac{\sqrt{\alpha}(\Delta k+1)}{2 \sqrt{\Delta k}}\right]-\operatorname{Erf}\left[\frac{\sqrt{\alpha}}{2 \sqrt{\Delta k}}\right]\right)\right]
\end{aligned}
$$

Al dar valores numéricos a $\Delta k=1$ y $\Delta q=1$ entonces podemos ver el comportamiento de la entropía en función de $\alpha$ en la figura 7.3 .

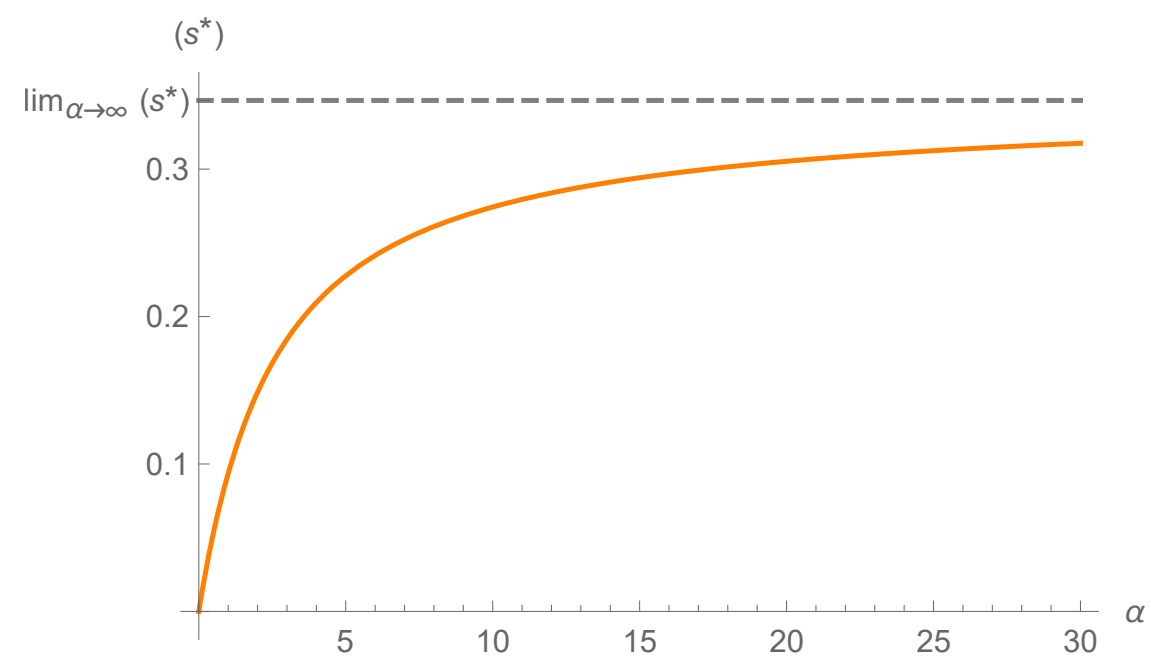

Figura 7.3: Entropía producida en el proceso de 1 a 2

El límite cuando $\alpha$ tiende a infinito es

$$
\lim _{\alpha \rightarrow \infty} \Delta\langle s\rangle_{1 \rightarrow 2}=\frac{\operatorname{Ln}[2]}{2}
$$




\subsection{Disminución de la intensidad del ruido}

Durante el segundo proceso, se realiza un cambio en la intensidad del ruido (6.7), mientras tanto la rigidez del potencial se mantiene constante 6.6. En este caso la solución de $\epsilon(t)$ está dada por (6.32) cuando tomamos en cuenta que el punto inicial corresponde al valor de epsilon evaluado en $\tau / 4$.

Utilizamos las expresiones encontradas para determinar las variables termodinámicas de este proceso. La solución la determinamos en función de las variables adimensionales $\Delta k, \Delta q$ y $\alpha$, además la condición inicial la reescribimos en términos de la constante $\varepsilon$,

$$
\epsilon\left(t_{0}\right)_{2 \rightarrow 3}=\frac{\gamma k_{1}}{m q_{2}}=\frac{M}{(1+\Delta q)}
$$

De esta manera como lo vimos en el proceso anterior la expresión del calor queda en términos de la expresión $\frac{k_{1}}{M}$, la cual tiene unidades de energía por lo que podemos escribir un calor adimensional $\frac{M\left\langle\Delta Q_{2 \rightarrow 3}\right\rangle}{k_{1}}=\left\langle\Delta Q_{2 \rightarrow 3}\right\rangle^{*}$

$$
\left\langle\Delta Q_{2 \rightarrow 3}\right\rangle^{*}=\frac{1}{16 \alpha}\left[-8 \Delta q\left(e^{-\frac{\alpha}{2}}-1\right)-4 \alpha \Delta q\right]
$$

La expresión es muy sencilla esto nos permite encontrar el límite del calor cuando se toma $\alpha$ muy grande sin la necesidad de dar valores numéricos.

$$
\lim _{\alpha \rightarrow \infty}\left\langle\Delta Q_{2 \rightarrow 3}\right\rangle^{*}=-\frac{\Delta q}{4}
$$

Damos los mismos valores numéricos a las variables adimensionales $\Delta k=1, \Delta q=$ 1 y obtenemos la gráfica mostrada en la figura 7.4

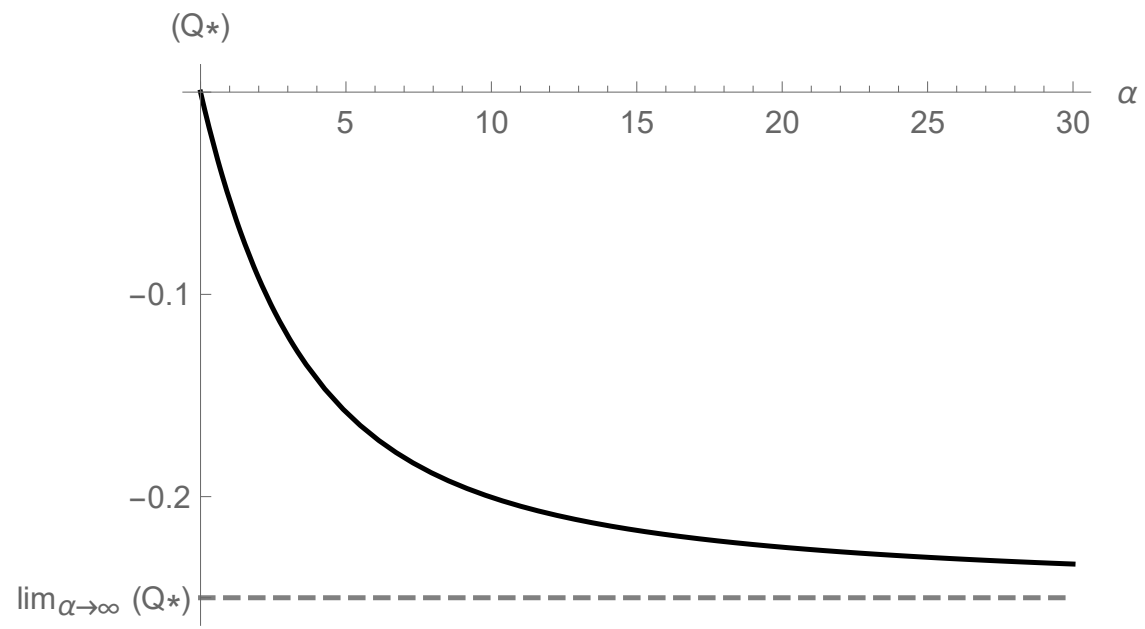

Figura 7.4: Calor durante el segundo proceso del ciclo, para $\Delta k=1$ y $\Delta q=1$

El cambio del calor en este proceso es negativo, esto implica que, durante el cambio de la intensidad del ruido, el sistema está dando calor al medio. Por otro lado, ya que la rigidez del potencial se mantiene contante durante todo el proceso no hay trabajo.

$$
\left\langle W_{2 \rightarrow 3}\right\rangle=0
$$


En este proceso el cambio de entropía lo encontramos utilizando la expresión 7.12 , en este caso el proceso se lleva a cabo durante el tiempo $t_{0}=\frac{\tau}{4}$ y $t_{f}=\frac{2 \tau}{4}$. Debemos de evaluar $\epsilon$ en los dos tiempos. Cuando evaluamos $\epsilon(t)$ en el tiempo inicial $t_{0}=\frac{\tau}{4}$ llegamos a la condición inicial.

$$
\frac{1}{\epsilon_{2 \rightarrow 3}\left(\frac{\tau}{4}\right)}=\frac{1}{\epsilon\left(t_{0}\right)_{2 \rightarrow 3}}
$$

y para el tiempo final tenemos

$$
\frac{1}{\epsilon_{2 \rightarrow 3}\left(\frac{2 \tau}{4}\right)}=-\frac{1}{\epsilon\left(t_{0}\right)} e^{-\frac{\alpha}{2}}-\frac{1}{\epsilon_{e q} \alpha(1+\Delta q)}\left(2 \Delta q\left[-1+e^{-\frac{\alpha}{2}}\right]-\alpha+\alpha e^{-\frac{\alpha}{2}}(1+\Delta q)\right)
$$

El cambio de entropía durante el proceso es el siguiente

$$
\begin{aligned}
\Delta\langle s\rangle_{2 \rightarrow 3} & =\frac{1}{2} \operatorname{Ln}\left[\epsilon ( t _ { 0 } ) \left(-\frac{1}{\epsilon\left(t_{0}\right)} e^{-\frac{\alpha}{2}}-\frac{1}{\epsilon_{e q} \alpha(1+\Delta q)}\left(2 \Delta q\left[-1+e^{-\frac{\alpha}{2}}\right]\right.\right.\right. \\
& \left.\left.\left.-\alpha+\alpha e^{-\frac{\alpha}{2}}(1+\Delta q)\right)\right)\right]
\end{aligned}
$$

Cuando $\alpha$ tiende a infinito entonces

$$
\lim _{\alpha \rightarrow \infty} \Delta\langle s\rangle_{2 \rightarrow 3}=\frac{1}{2} \operatorname{Ln}\left[\frac{\epsilon\left(t_{0}\right)_{2 \rightarrow 3}}{\epsilon_{e q}(1+\Delta q)}\right]
$$

Tomando el caso en que $\epsilon\left(t_{0}\right)=\epsilon_{e q}$ el límite se reduce a

$$
\lim _{\alpha \rightarrow \infty} \Delta\langle s\rangle_{2 \rightarrow 3}=\frac{1}{2} \operatorname{Ln}\left[\frac{1}{(1+\Delta q)}\right]
$$

Y la gráfica de la entropía dados los valores $\Delta k=1$ y $\Delta q=1$ queda de la siguiente forma 7.5 .

\subsection{Aumento del potencial}

En esta sección del ciclo se realiza un aumento de la rigidez del potencial 6.8 manteniendo una intensidad de ruido constante $(6.9)$. La solución a la distribución de probabilidad depende de la forma de $\epsilon(t)$ que en este caso tiene la forma de 6.34). Con el mismo análisis adimensional que hemos hecho en los otros procesos notamos que podemos escribir una expresión adimensional para el calor en este proceso $\left\langle\Delta Q_{3 \rightarrow 4}\right\rangle^{*}=\frac{M\left\langle Q_{3 \rightarrow 4}\right\rangle}{k_{1}}$

$$
\left\langle Q_{3 \rightarrow 4}\right\rangle^{*}=-\frac{e^{\frac{\alpha}{4 \Delta k}}}{4}\left\{(\Delta k+1) e^{-\frac{\alpha}{4 \Delta k}(\Delta k+1)^{2}}+\frac{\sqrt{\Delta k} \sqrt{\pi}}{\sqrt{\alpha}} \operatorname{Erf}\left(\frac{\sqrt{\alpha}}{2 \sqrt{\Delta k}}(\Delta k+1)\right)\right.
$$




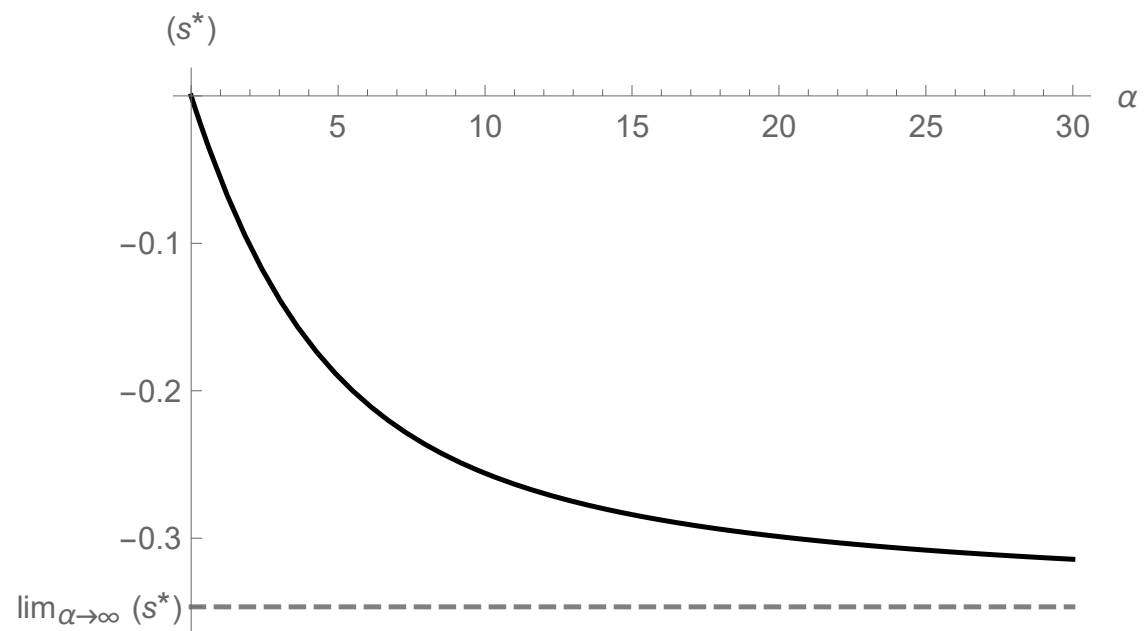

Figura 7.5: Entropía durante el proceso de 2 a 3.

$$
\begin{aligned}
& \left.+e^{-\frac{\alpha}{4 \Delta k}}-\frac{\sqrt{\Delta k} \sqrt{\pi}}{\sqrt{\alpha}} \operatorname{Erf}\left(\frac{\sqrt{\alpha}}{2 \sqrt{\Delta k}}\right)\right\}+\frac{\sqrt{\alpha} \sqrt{\pi}}{8 \sqrt{\Delta k}} \operatorname{Erfi}\left(\frac{\sqrt{\alpha}}{2 \sqrt{\Delta k}}\right) \\
& \left\{(\Delta k+1) e^{-\frac{\alpha}{4 \Delta k}(\Delta k+1)^{2}}+\frac{\sqrt{\Delta k} \sqrt{\pi}}{\sqrt{\alpha}} \operatorname{Erf}\left(\frac{\sqrt{\alpha}}{2 \sqrt{\Delta k}}(\Delta k+1)\right)+e^{-\frac{\alpha}{4 \Delta k}}\right. \\
& \left.-\frac{\sqrt{\Delta k} \sqrt{\pi}}{\sqrt{\alpha}} \operatorname{Erf}\left(\frac{\sqrt{\alpha}}{2 \sqrt{\Delta k}}\right)\right\}-\frac{\alpha}{16 \Delta k}\left\{(\Delta k+1)^{2}(1\right. \\
& \left.\left.-{ }_{2} F_{2}\left[1,1 ; \frac{1}{2}, 2 ;-\frac{\alpha}{4 \Delta k}(\Delta k+1)^{2}\right]\right)-\left(1-{ }_{2} F_{2}\left[1,1 ; \frac{1}{2}, 2 ;-\frac{\alpha}{4 \Delta k}\right]\right)\right\} \\
& +\frac{\alpha(\Delta k+2)}{16}
\end{aligned}
$$

Esta expresión depende únicamente de $\Delta k$ y de $\alpha$, para analizarla damos un valor numérico a la diferencia de rigidez de la trampa.

$$
\Delta k=1
$$

De esta manera podemos encontrar el límite cuando $\alpha$ tiende a infinito

$$
\lim _{\alpha \rightarrow \infty}\left\langle Q_{3 \rightarrow 4}\right\rangle^{*}=-\frac{\operatorname{Ln}[2]}{4}
$$

Graficamos para determinar el comportamiento del calor cuando $\Delta k=1$ en función de $\alpha$ como se muestra en la figura 7.6

El calor en este proceso es negativo lo cual implica que el aumento del potencial conlleva a un flujo de calor de la partícula Browniana al medio, mientras que existe un trabajo debido al mismo. El cambio en la rigidez del potencial es

$$
\dot{k}(t)=\frac{d k(t)}{d t}=\frac{k_{2}-k_{1}}{\tau} 4
$$

Este cambio de potencial nos lleva a encontrar la expresión del trabajo, así como lo hicimos en la disminución del potencial tomamos como condición inicial que el 


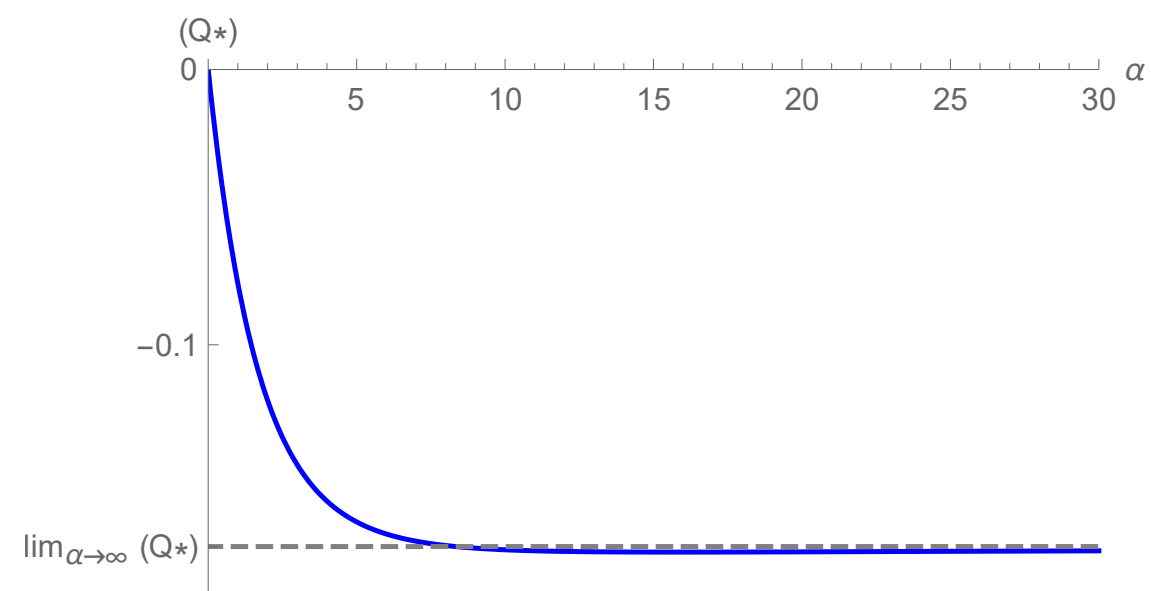

Figura 7.6: Calor durante el aumento del potencial(Proceso de 3 a 4).

sistema se encuentra en equilibrio, escribimos el valor del trabajo adimensional $\left\langle W_{3 \rightarrow 4}\right\rangle^{*}=\frac{M\left\langle W_{3 \rightarrow 4}\right\rangle}{k_{1}}$

$$
\begin{aligned}
\left\langle W_{3 \rightarrow 4}\right\rangle^{*} & =-\frac{\sqrt{\pi} \Delta k}{4 \sqrt{\alpha} \sqrt{\Delta k}}\left\{e^{\frac{\alpha}{4 \Delta k}}\left(\operatorname{Erf}\left[\frac{\sqrt{\alpha}}{2 \sqrt{\Delta k}}\right]-\operatorname{Erf}\left[\frac{\sqrt{\alpha}(1+\Delta k)}{2 \sqrt{\Delta k}}\right]\right)\right. \\
& -\frac{2 \alpha}{8 \sqrt{\pi} \sqrt{\alpha} \Delta k^{3 / 2}}\left(2 \pi \Delta k \operatorname{Erf}\left[\frac{\sqrt{\alpha}}{2 \sqrt{\Delta k}}\right] \operatorname{Erfi}\left[\frac{\sqrt{\alpha}}{2 \sqrt{\Delta k}}\right]\right. \\
& -\alpha F\left[(1,1),\left(\frac{3}{2}, 2\right),-\frac{\alpha}{4 \Delta k}\right]-2 \pi \Delta k \operatorname{Erf}\left[\frac{\sqrt{\alpha}(1+\Delta k)}{2 \sqrt{\Delta k}}\right] \operatorname{Erfi}\left[\frac{\sqrt{\alpha}}{2 \sqrt{\Delta k}}\right] \\
& \left.\left.+\alpha(1+\Delta k)^{2} F\left[(1,1),\left(\frac{3}{2}, 2\right),-\frac{\alpha(1+\Delta k)^{2}}{4 \Delta k}\right]\right)\right\}
\end{aligned}
$$

Así el trabajo al igual que el que hemos determinado anteriormente, depende únicamente de $\Delta k$, y de la variable adimensional $\alpha=\frac{k_{1} \tau}{m \gamma}$. Para analizar la solución damos los mismos valores adimensionales, de esta manera podemos encontrar el límite y graficar con respecto a $\alpha$. El límite cuando $\alpha$ tiende a infinito es

$$
\lim _{\alpha \rightarrow \infty}\left\langle W_{3 \rightarrow 4}\right\rangle^{*}=\frac{\operatorname{Ln}[2]}{4}
$$

La figura 7.7 nos indica el comportamiento del trabajo, en este caso el trabajo positivo se está haciendo sobre la partícula Browniana, solo recupera el valor del límite cuando $\alpha$ es infinito.

En el límite, el calor liberado es en magnitud igual al trabajo ejercido, esto implica de nuevo el cumplimiento de la primera ley para un gas ideal $\Delta W=\Delta Q$. Para la entropía analizamos la solución 6.34 en el tiempo inicial y final del proceso. En este caso el tiempo va de $t_{0}=\frac{2 \tau}{4}$ a $t_{f}=\frac{3 \tau}{4}$ la solución en el tiempo inicial está dado por la condición inicial y para el tiempo final llegamos a

$$
\frac{1}{\epsilon_{3 \rightarrow 4}\left(\frac{2 \tau}{4}\right)}=\frac{1}{\epsilon\left(t_{0}\right)}
$$




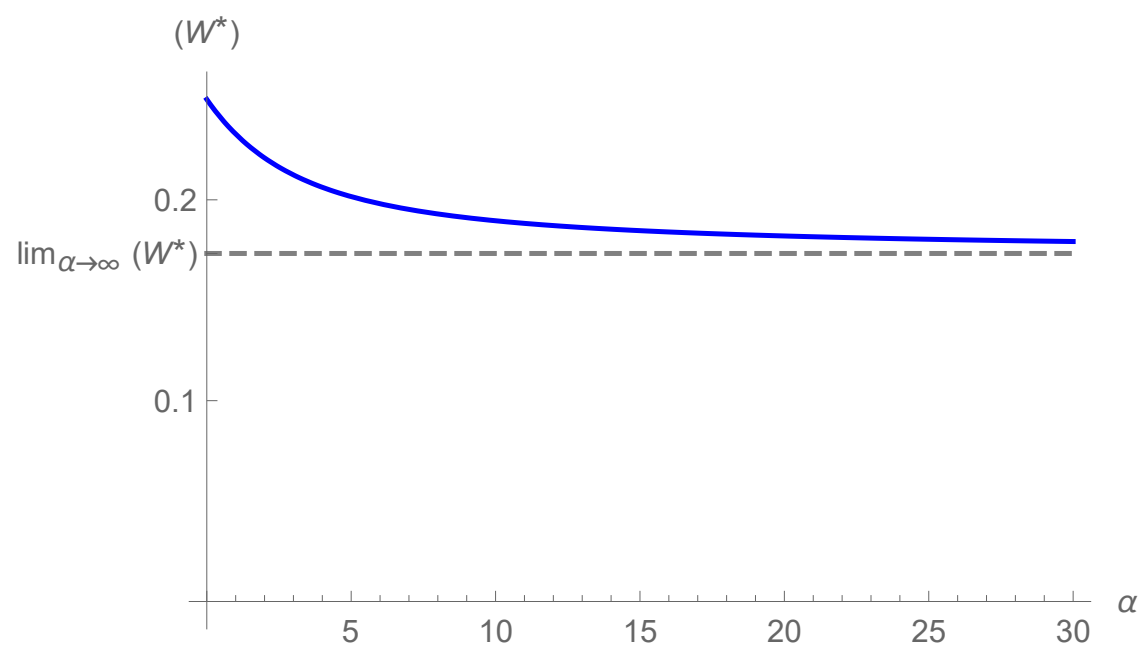

Figura 7.7: Gráfica del trabajo durante el proceso 3 a 4 con $\Delta k=1$ y $\Delta q=1$

y para el tiempo final

$$
=\frac{1}{\epsilon\left(t_{0}\right)_{3 \rightarrow 4}} e^{-\frac{\alpha\left(\frac{\Delta k}{2}+1\right)}{2}}+\frac{\sqrt{\alpha} \sqrt{\pi} e^{-\frac{\alpha}{4 \Delta k}(\Delta k+1)^{2}}}{2 \epsilon_{e q} \sqrt{\Delta k}}\left[\operatorname{Erfi}\left(\frac{\sqrt{\alpha}}{2 \sqrt{\Delta k}}\right)-\operatorname{Erfi}\left(\frac{\sqrt{\alpha}(\Delta k+1)}{2 \sqrt{\Delta k}}\right)\right.
$$

Tomando esto en cuenta entonces el cambio de entropía durante el proceso es el siguiente

$$
\begin{aligned}
\Delta\langle s\rangle_{3 \rightarrow 4} & =\frac{1}{2} \operatorname{Ln}\left[\frac{\epsilon\left(t_{o}\right)}{\epsilon\left(t_{f}\right)}\right] \\
& =\frac{1}{2} \operatorname{Ln}\left[\epsilon ( t _ { 0 } ) \left(\frac{1}{\epsilon\left(t_{0}\right)} e^{-\frac{\alpha\left(\frac{\Delta k}{2}+1\right)}{2}}+\frac{\sqrt{\alpha} \sqrt{\pi} e^{-\frac{\alpha}{4 \Delta k}(\Delta k+1)^{2}}}{2 \epsilon_{e q} \sqrt{\Delta k}}\right.\right. \\
& {\left.\left.\left[\operatorname{Erfi}\left(\frac{\sqrt{\alpha}}{2 \sqrt{\Delta k}}\right)-\operatorname{Erfi}\left(\frac{\sqrt{\alpha}(\Delta k+1)}{2 \sqrt{\Delta k}}\right)\right]\right)\right] }
\end{aligned}
$$

Cuando $\epsilon\left(t_{0}\right)_{3 \rightarrow 4}=\epsilon_{e q}$ y damos valores numéricos a $\Delta k_{1}=1$ y $\Delta q_{1}=1$ encontramos que el límite cuando $\alpha$ tiende a infinito es

$$
\lim _{\alpha \rightarrow \infty} \Delta\langle s\rangle_{3 \rightarrow 4}=-\frac{\operatorname{Ln}[2]}{2}
$$

y al graficar el comportamiento de este encontramos su decaimiento al valor límite como vemos en l figura 7.8

\subsection{Aumento de la intensidad del ruido}

El último proceso del ciclo es un cambio en la intensidad del ruido (6.11) manteniendo la rigidez del potencial constante (6.10). La forma de $\epsilon(t)$ durante este proceso está tada por 6.36.

En este proceso la expresión del calor podemos reescribirla como un calor adi- 


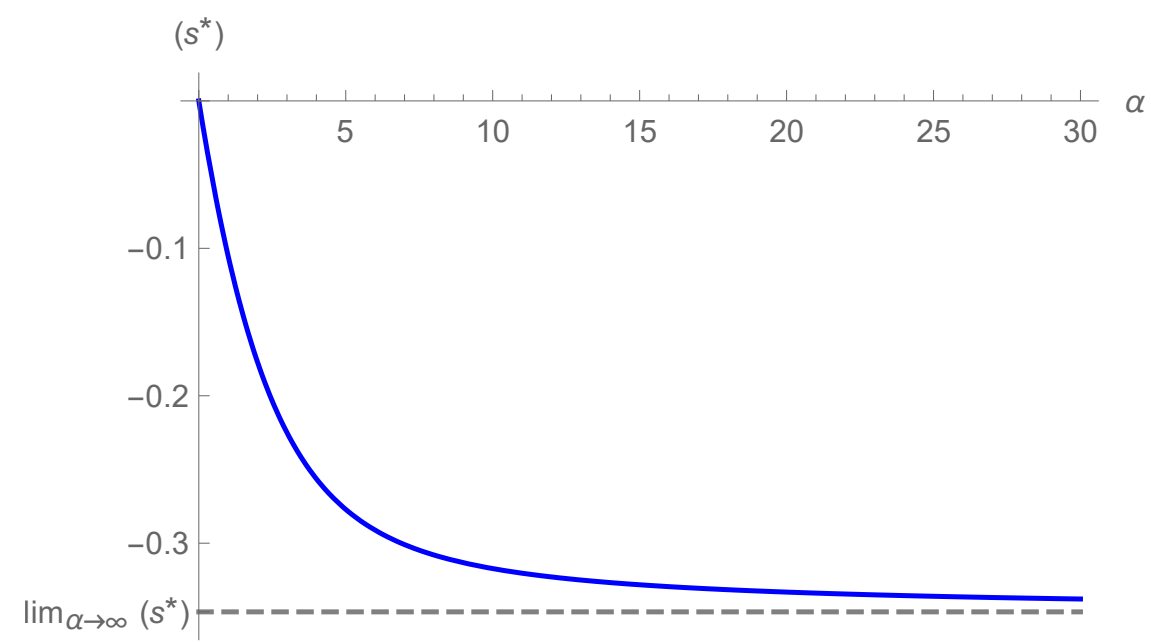

Figura 7.8: Cambio de entropía durante el proceso de 3 a 4

mensional $\frac{M\left\langle\Delta Q_{4 \rightarrow 1}\right\rangle}{k_{1}}$

$$
\left\langle\Delta Q_{4 \rightarrow 1}\right\rangle^{*}=\frac{\Delta q\left(e^{-\frac{\alpha}{2}(1+\Delta k)}+1\right)}{2 \alpha(1+\Delta k)}+\frac{\Delta q}{4}
$$

El límite cuando $\alpha$ tiende a infinito

$$
\lim _{\alpha \rightarrow \infty}\left\langle\Delta Q_{4 \rightarrow 1}\right\rangle^{*}=\frac{\Delta q}{4}
$$

Dando valor a $\Delta q=1$ y $\Delta k=1$ podemos graficar obteniendo el comportamiento graficado en la figura 7.9

$(Q *)$

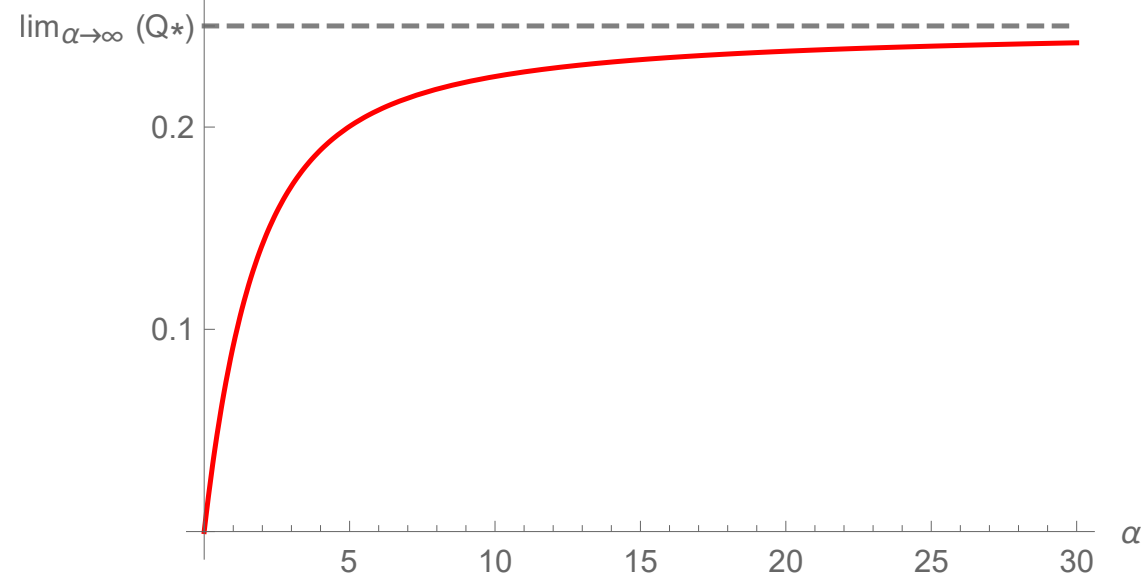

Figura 7.9: Calor durante el proceso de 4 a 1

El flujo de calor en este caso va del medio a la partícula Browniana y debido a que el proceso se realiza manteniendo la rigidez del potencial constante,

$$
\frac{d k(t)}{d t}=0
$$


no hay trabajo

$$
\langle W\rangle=0
$$

La entropía se encuentra analizando $\epsilon$ que en este caso el proceso se realiza en un tiempo que va de $t_{0}=\frac{3 \tau}{4}$ a $t_{f}=\tau$ al evaluar la solución en cada uno de los tiempos encontramos que en el tiempo inicial tenemos el valor dado de la condición inicial y para el tiempo final tenemos

$$
\begin{aligned}
\frac{1}{\epsilon_{4 \rightarrow 1}(t)} & =\frac{e^{-(1+\Delta k)\left(\frac{\alpha}{2}\right)}}{\epsilon\left(t_{0}\right)}+\frac{1}{\epsilon_{e q} \alpha(1+\Delta k)}[4 \alpha \Delta q(1+\Delta k) \\
& \left.-\left(-2 \Delta q\left(-1+e^{(1+\Delta k)\left(-\frac{\alpha}{2}\right)}\right)+\alpha(1+\Delta k)\left(-1+e^{(1+\Delta k)\left(-\frac{\alpha}{2}\right)}+3 \Delta q\right)\right)\right]
\end{aligned}
$$

Tomando esto en cuenta podemos escribir la diferencia de entropía en el proceso

$$
\begin{aligned}
\Delta\langle s\rangle_{4 \rightarrow 1} & =\frac{1}{2} \operatorname{Ln}\left[\frac{\epsilon\left(t_{o}\right)}{\epsilon\left(t_{f}\right)}\right]=\frac{1}{2} \operatorname{Ln}\left[\frac{\epsilon\left(t_{0}\right) e^{-(1+\Delta k)\left(\frac{\alpha}{2}\right)}}{\epsilon\left(t_{0}\right)}\right. \\
& +\frac{\epsilon\left(t_{0}\right)}{\epsilon_{e q} \alpha(1+\Delta k)}\left[4 \alpha \Delta q(1+\Delta k)-\left(-2 \Delta q\left(-1+e^{(1+\Delta k)\left(-\frac{\alpha}{2}\right)}\right)\right.\right. \\
& \left.\left.\left.+\alpha(1+\Delta k)\left(-1+e^{(1+\Delta k)\left(-\frac{\alpha}{2}\right)}+3 \Delta q\right)\right)\right]\right]
\end{aligned}
$$

En el límite cuando $\alpha$ tiende a infinito encontramos

$$
\lim _{\alpha \rightarrow \infty} \Delta\langle s\rangle_{4 \rightarrow 1}=\frac{1}{2} \operatorname{Ln}\left[\frac{\epsilon\left(t_{0}\right)}{\epsilon_{e q}}(\Delta q+1)\right]
$$

En el caso en el que $\epsilon\left(t_{0}\right)=\epsilon_{e q}$ y damos valores numéricos a $\Delta k_{1}=1$ y $\Delta q_{1}=1$ encontramos el comportamiento de la eficiencia en función de $\alpha$ que se muestra en la figura 7.10 .

$\left(s^{*}\right)$

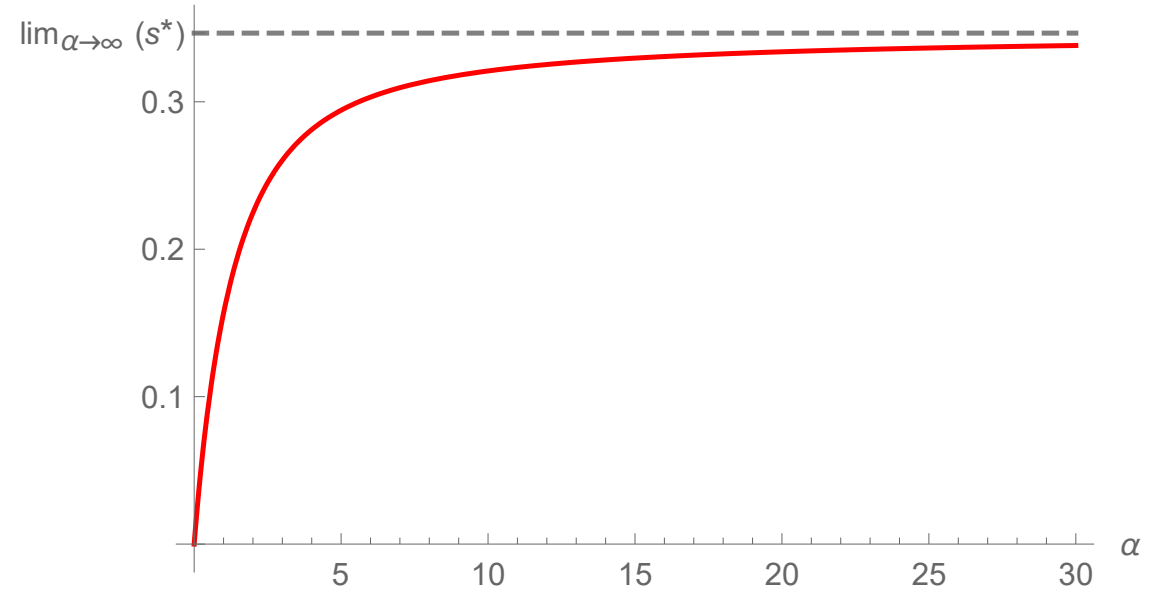

Figura 7.10: Cambio en la entropía durante el proceso de 4 a 1

La entropía se aproxima de forma rápida a su límite, sin embargo solo llega a éste cuando $\alpha$ tiende a infinito. 


\subsection{Análisis}

Durante el ciclo en los cuatro procesos existe una transferencia de energía entre el medio y la partícula, al que llamamos calor, el signo nos indica si el calor está siendo absorbido por la partícula o si está liberando al medio. En nuestra convención de signos un calor positivo significa que el sistema está recibiendo calor del medio, calor negativo indica lo contrario. Como podemos ver en la figura 7.11, durante el proceso de 1 a 2 cuando disminuye la rigidez manteniendo una intensidad de ruido constante (línea amarilla) y el proceso de 4 a 1 cuando la intensidad del ruido aumenta con una rigidez de la trampa constante (línea roja) tenemos un calor positivo, esto implica que durante estos procesos se está absorbiendo calor, durante los otros dos procesos se está liberando calor al medio.

$(\mathrm{Q})$

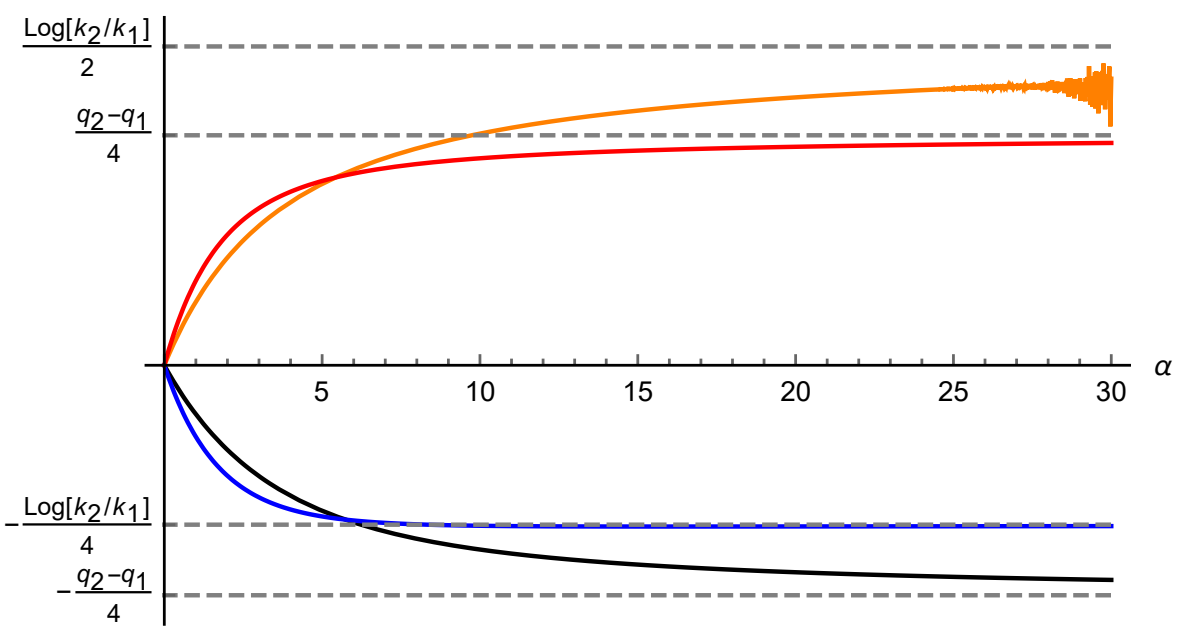

Figura 7.11: Gráfica del calor durante todo el ciclo, la línea naranja corresponde a la disminución del potencial, la negra corresponde a la disminución de la intensidad del ruido, la azul al aumento del potencial y la roja concierne al aumento de la intensidad del ruido.

Tomando en cuenta solo los límites cuando $\alpha$ es muy grande y los valores otorgados a $\Delta k$ y $\Delta q$ podemos ver que al sumar los calores $\left\langle\Delta Q_{3 \rightarrow 3}\right\rangle^{*}$ y $\left\langle\Delta Q_{4 \rightarrow 1}\right\rangle^{*}$ se eliminan sin importar el valor de las $k$ y $q$ y al final del proceso

$$
\begin{aligned}
& \left\langle\Delta Q_{1 \rightarrow 2}\right\rangle^{*}+\left\langle\Delta Q_{2 \rightarrow 3}\right\rangle^{*}+\left\langle\Delta Q_{3 \rightarrow 4}\right\rangle^{*}+\left\langle\Delta Q_{4 \rightarrow 1}\right\rangle^{*} \\
& =-\frac{\operatorname{Ln}[2]}{4}+\frac{\operatorname{Ln}[2]}{2}=\frac{\operatorname{Ln}[2]}{4}
\end{aligned}
$$

El calor final es positivo, esto indica que el sistema está absorbiendo calor.

Durante el ciclo hay dos procesos en los que existe trabajo como mencionamos antes, estos los podemos ver en la figura 7.12, el signo nos indica si el trabajo está siendo ejercido sobre la partícula o si la partícula está haciendo trabajo sobre el sistema. En nuestra convención de signos, el positivo indica que se está haciendo trabajo sobre la partícula y el negativo indica lo contrario. En el primer proceso 
del ciclo cuando se disminuye la rigidez del potencial para limitar el movimiento el trabajo es negativo, es decir al reducir la rigidez del potencial existe un trabajo que realiza la partícula sobre el sistema. En el proceso de 3 a 4 cuando se aumenta la rigidez del potencial se tiene un trabajo positivo que se está ejerciendo sobre el sistema.

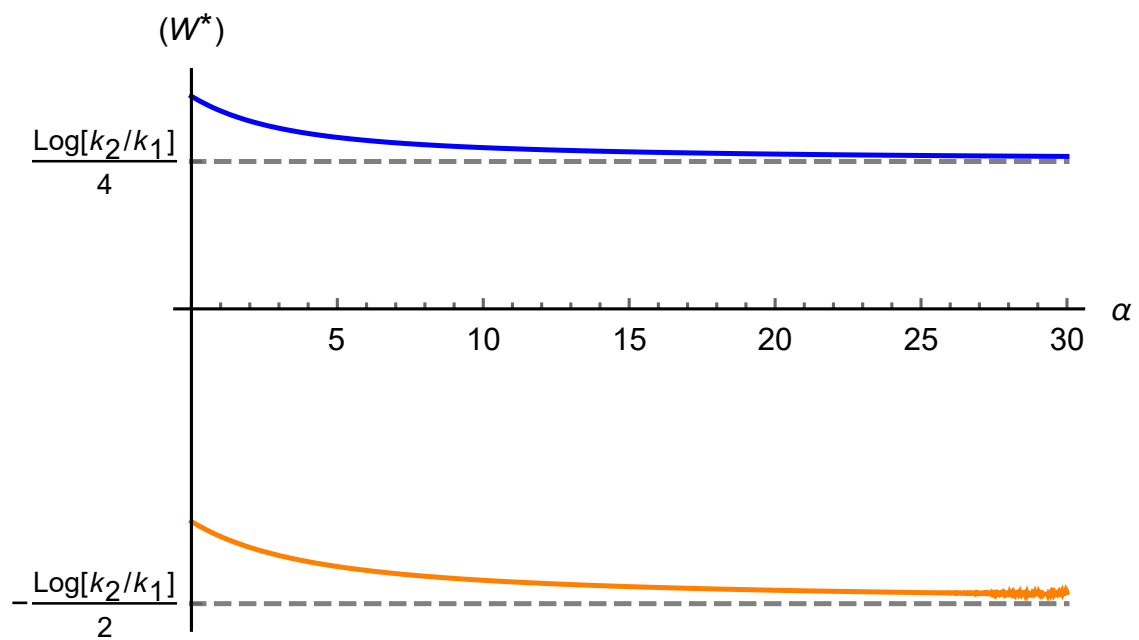

Figura 7.12: Gráfica del trabajo durante todo el ciclo

Podemos notar que el valor absoluto del trabajo ejercido sobre el sistema (el que es positivo) siempre es menor que el valor absoluto del trabajo generado (el que es negativo). En el límite obtenemos

$$
\langle\Delta W\rangle_{t o t}^{*}=\left\langle\Delta W_{1 \rightarrow 2}\right\rangle^{*}+\left\langle\Delta W_{3 \rightarrow 4}\right\rangle^{*}=-\frac{\operatorname{Ln}[2]}{4}
$$

Por lo tanto, hay trabajo generado. Siguiendo los postulados de la termodinámica clásica, para que esto ocurra debe de haber calor absorbido por el sistema y como lo hemos visto esto efectivamente sucede. La máquina Browniana absorbe calor del medio y genera trabajo.

Durante la realización de los 4 procesos vemos en la figura 7.13 que la forma de la entropía tiende al valor en equilibrio, sin embargo, nunca llega a este estado. En el límite cuando $\alpha$ tiende a infinito, la máquina se comporta como un ciclo ideal en el que el total de la variación de la entropía es nula, solo en este caso el ciclo es reversible.

La eficiencia térmica de la máquina se representa como la fracción del calor absorbido que se convierte en trabajo. El cociente está dado por el trabajo total $W$ efectuado por la partícula Browniana, sobre el calor suministrado a la máquina.

$$
\eta=\frac{\left|W_{t o t}\right|}{Q_{a b s}}
$$

El trabajo en el ciclo es

$$
W_{t o t}=\left\langle\Delta W_{1 \rightarrow 2}^{*}\right\rangle+\left\langle\Delta W_{3 \rightarrow 4}^{*}\right\rangle
$$




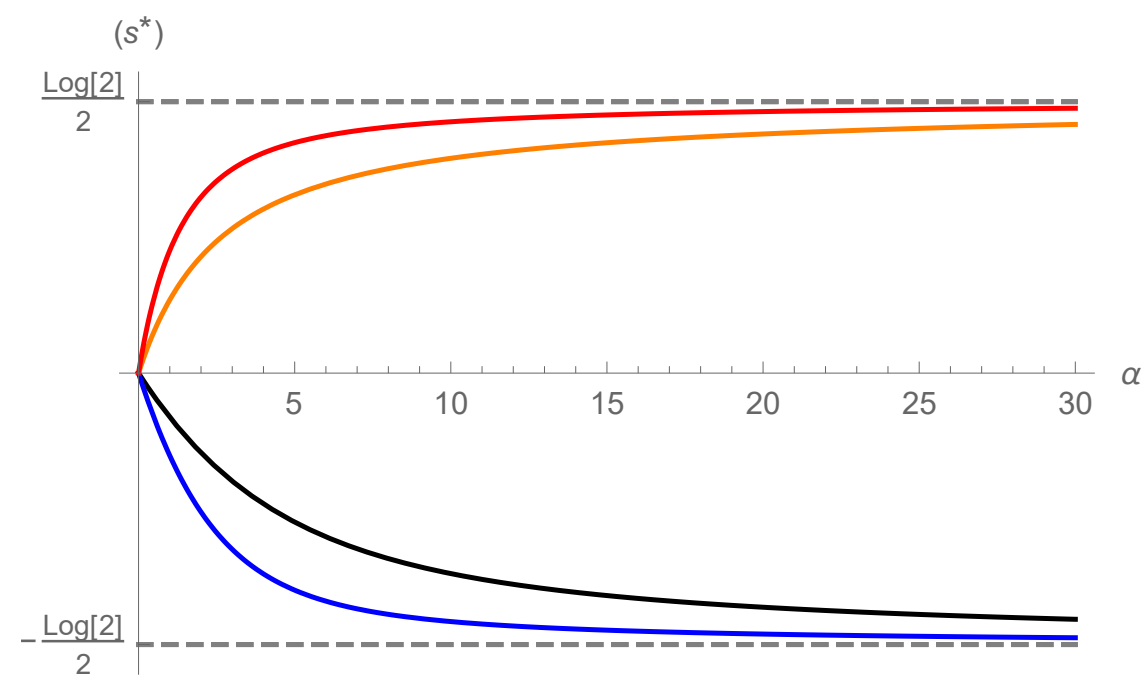

Figura 7.13: Cambio en la entropía durante el ciclo.

Evaluado en el límite cuando $\alpha$ tiende a infinito el valor del cambio de trabajo total es

$$
W_{t o t}=-\frac{\operatorname{Ln}[2]}{2}+\frac{\operatorname{Ln}[2]}{4}=-\frac{\operatorname{Ln}[2]}{4}
$$

El trabajo total es negativo, el signo indica que es un trabajo que realiza la partícula Browniana sobre sus alrededores. En el caso del calor absorbido notamos que durante la disminución de la rigidez del potencial el flujo de energía va del medio a la partícula Browniana $\left\langle\Delta Q_{1 \rightarrow 2}^{*}\right\rangle$ pero no sólo eso, durante los procesos $\left\langle\Delta Q_{2 \rightarrow 3}^{*}\right\rangle$ y $\left\langle\Delta Q_{4 \rightarrow 2}^{*}\right\rangle$ existe calor, un calor absorbido que en el límite cuando $\alpha$ es infinito se elimina y nos regresa al caso ideal.

$$
\begin{aligned}
Q_{a b s} & =\left\langle\Delta Q_{1 \rightarrow 2}^{*}\right\rangle+\left\langle\Delta Q_{2 \rightarrow 3}^{*}\right\rangle+\left\langle\Delta Q_{3 \rightarrow 4}^{*}\right\rangle \\
& =\frac{\operatorname{Ln}[4]}{2}-\frac{\Delta q}{4}+\frac{\Delta q}{4}=\frac{\operatorname{Ln}[4]}{2}
\end{aligned}
$$

Tomando esto en cuenta la eficiencia en el límite para $\Delta k=1$ y $\Delta q=1$ es

$$
\eta=\frac{1}{2}
$$

obtenemos la eficiencia ideal de un ciclo de Stirling que es la misma que la de una máquina de Carnot ideal. Pero tomando en cuenta $\alpha$ pequeñas esperamos que la eficiencia sea menor que la del límite, para esto utilizamos las expresiones ya encontradas del calor (7.14), (7.24), (7.33) y del trabajo (7.18) y (7.35). Damos valores numéricos a $\Delta k=1$ y $\Delta q=1$ para determinar la forma de la eficiencia del ciclo en función de $\alpha$ dada por la ecuación (7.56

$$
\eta=\frac{\left|\left\langle\Delta W_{1 \rightarrow 2}^{*}\right\rangle+\left\langle\Delta W_{3 \rightarrow 4}^{*}\right\rangle\right|}{\left\langle\Delta Q_{1 \rightarrow 2}^{*}\right\rangle+\left\langle\Delta Q_{2 \rightarrow 3}^{*}\right\rangle+\left\langle\Delta Q_{3 \rightarrow 4}^{*}\right\rangle}
$$

Efectivamente vemos que el protocolo propuesto nos ha llevado a la realización de un ciclo de Stirling, de esta manera se genera trabajo a partir de un calor absorbido y en el caso límite su comportamiento es idéntico al del ciclo de Stirling para un gas ideal. Cuando nos quedamos fuera del caso límite la eficiencia 


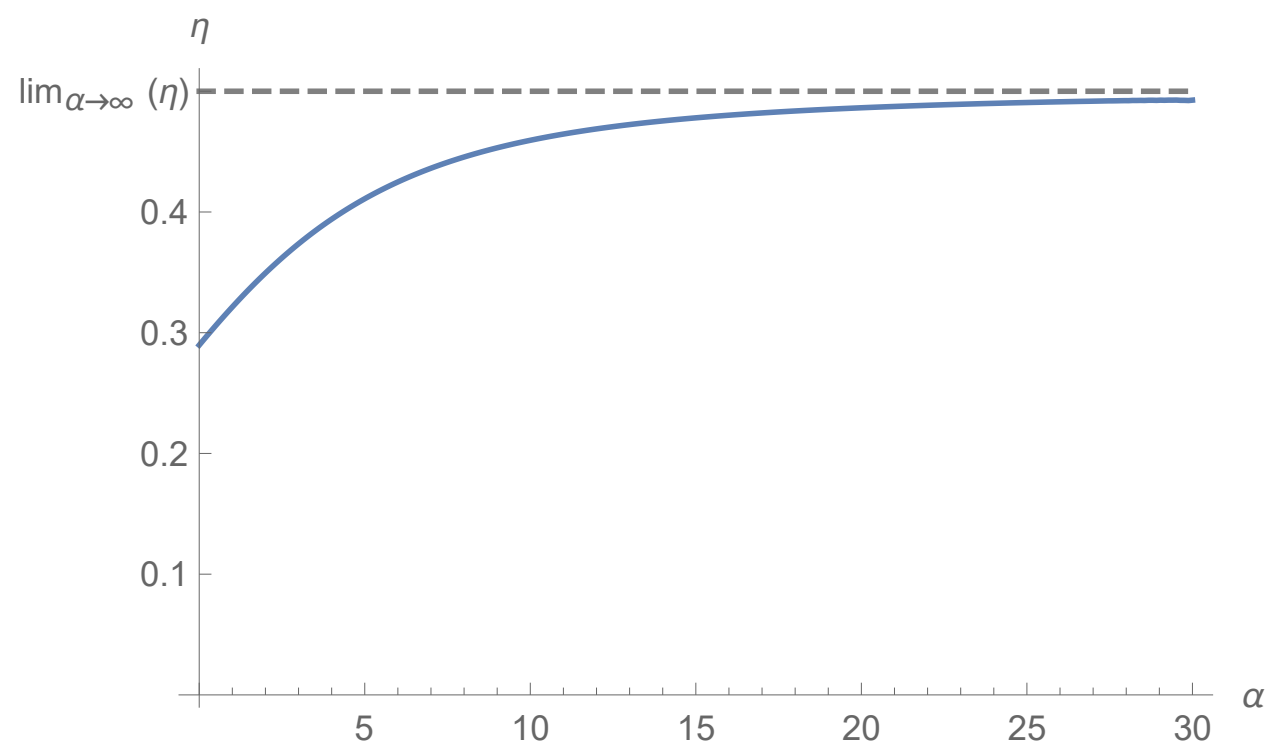

Figura 7.14: Grafica de la eficiencia del ciclo dada por la ecuación 7.56 dando los valores numéricos a las variables adimensionales $\Delta k=1$ y $\Delta q=1$.

es menor, el total de la entropía es diferente a cero, pero sigue persistiendo un trabajo realizado a partir del calor absorbido. Para confirmar tomamos un valor de $\alpha$ que no es muy grande para que no se encuentre cerca de los límites.

Para $\alpha=5$ :

El cambio de entropía en el ciclo está dado por $\Delta S=0.294268$, cuando es diferente de cero nos indica que estamos en un ciclo que ya no es reversible, y efectivamente cumple con la premisa de que la entropía del sistema siempre aumenta, mientras más pequeño sea $\alpha$ más grande será la entropía, cada vez nos estaríamos alejando más del caso de equilibrio en el que el ciclo es reversible. El calor total del ciclo para este caso es $\Delta Q=0.0749527$ en este caso el calor es positivo por lo que, el flujo de éste va del medio a la partícula. Finalmente, el trabajo total en el ciclo para este caso está dado por $\Delta W=-0.101376$ esto implica que se está generando calor. Vemos que efectivamente para cada valor de $\alpha$ se cumple que tenemos una máquina térmica de una partícula Browniana, pero que ya no es reversible y se encuentra fuera del equilibrio. 


\section{Capítulo 8}

\section{Conclusiones y perspectivas}

A lo largo de este trabajo utilizando la termodinámica estocástica para sistemas micrométricos desarrollada por Sekimoto y la teoría basada en el desarrollo de las pinzas ópticas, pudimos proponer un protocolo con el cual se fuerza a la partícula Browniana para que recorra un ciclo de Stirling en un tiempo $\tau$ finito. Con esta hipótesis, se le está sacando del equilibrio térmico con el baño y por lo tanto el estudio de este sistema debe de ser un estudio completamente fuera de equilibrio y no se debe de usar el teorema de fluctuación disipación, el cual que casi siempre se toma como axioma. Tomando esto en cuenta proponemos un sistema en una dimensión, manipulado externamente por un potencial tipo oscilador armónico. Partimos de la ecuación de Langevin sobreamortiguada para encontrar la ecuación de movimiento de una partícula en cada uno de los procesos que componen al ciclo. La simulación de la ecuación de Langevin nos permitió ver el comportamiento de la partícula cuando se modifican los parámetros externos que logran que ésta realice el ciclo. A partir de la ecuación de Langevin determinamos la ecuación de Fokker-Planck y propusimos una distribución de probabilidad Gaussiana en función de una función del tiempo $\epsilon(t)$ que está relacionada con la varianza y es diferente dependiendo del proceso que se vaya a evaluar.

La densidad de probabilidad se utilizó para realizar el análisis termodinámico basado en las ecuaciones derivadas del estudio de Sekimoto et al. Los resultados de este análisis mostraron que la partícula al ser manipulada por el protocolo propuesto efectivamente hace la función de una máquina térmica cuyo flujo de energía (calor) total va del medio a la partícula Browniana y parte se convierte en trabajo que realiza la partícula Browniana sobre el medio, todo ello de acuerdo con la primera ley de la termodinámica. Así mismo mediante el trabajo de Seifert et al es posible definir la entropía para una trayectoria de una partícula Browniana y su eficiencia, al determinarla encontramos una concordancia con la segunda ley de la termodinámica y respeta el límite de eficiencia del ciclo de Carnot.

Las soluciones se obtuvieron en términos de variables adimensionales $\Delta k, \Delta q$ y $\alpha$, en especial $\alpha$ nos indica el cociente entre dos tiempos importantes del sistema, en esta variable adimensional se puede comparar el tiempo del ciclo $\tau$ con el tiempo característico del sistema $\frac{k_{1}}{m \gamma}$, de manera que para $\alpha$ grande implica que $\tau$ es muy grande comparado con $\frac{k_{1}}{m \gamma}$ o que $\tau$ es muy chica comparada con $\frac{k_{1}}{m \gamma}$. Esto nos permitio evaluar las soluciones en sus casos límites, tomando $\alpha$ tendiendo a 
infinito pudimos encontrar que las soluciones encontradas son las mismas que las del caso en cuasi-equilibrio y que además tiene una similitud muy cercana a la solución de un gas ideal en termodinámica clásica, esto significa que si el proceso se realiza en un tiempo infinito podríamos regresar al caso ideal reversible y por lo tanto tomar en cuenta que el sistema se encuentra en equilibrio con el medio, por otro lado esto también sucede si por ejemplo la fricción del medio es muy pequeña comparada con el tamaño de $\frac{k_{1} \tau}{m}$, etc. Y esto no es todo, también pudimos comprobar que para cualquier otro $\alpha$ el análisis basado en el desarrollo de la termodinámica de Sekimoto, el ciclo sigue cumpliendo la función de una máquina y la entropía del sistema nos indica que el sistema no se encuentra en equilibrio y por lo tanto se trata de un ciclo irreversible cuya eficiencia es menor que la del caso ideal de equilibrio.

Es decir, obtuvimos una solución para el ciclo de Stirling que nos permite determinar los valores del calor, el trabajo y la entropía para cualquier $\tau$ y efectivamente estos valores difieren del caso límite cuando $\tau$ tiende a infnito.

Tomando en cuenta los valores numéricos del experimento realizado por Martínez encontramos un valor aproximado de $\alpha$ dado por 2647.28 en este caso $\alpha$ es tan grande que el análisis tomando en cuenta un cuasi-equilibrio es una buena aproximación.

Este trabajo solo es el punto de partida de una gran variedad de trabajos que se pueden realizar.

1. Realizar un análisis numérico del ciclo, pero tomando en cuenta que solo el inicio del ciclo está un estado de equilibrio.

2. Realizar el mismo análisis con un protocolo diferente.

3. Determinar hasta qué punto el trabajo, el calor, entropía etc. dependen del protocolo.

4. Realizar un análisis de la reversibilidad del ciclo utilizando los teoremas de fluctuación.

5. Hacer el mismo análisis con otros ciclos. 



\section{Apéndice A}

\section{Análisis dimensional}

El sistema de estudio está formado por una partícula Browniana inmersa en un fluido y en presencia de un potencial armónico, por lo tanto su dinámica depende de las siguientes cantidades: $k(t)$ la rigidez del potencial, $\gamma$ es el coeficiente de fricción por unidad de masa, $\epsilon(t)$, es parte de la solución de la distribución de probabilidad y está relacionado con la varianza, $q(t)$ es la intensidad del ruido y $t$ es el tiempo en el que se está evaluando. Las dimensiones de cada uno son las siguientes:

$$
\begin{aligned}
& {[k(t)]=\frac{k g}{s^{2}}} \\
& {[\gamma]=\frac{1}{s}} \\
& {[\epsilon(t)]=\frac{1}{m^{2}}} \\
& {[q(t)]=\frac{m^{2}}{s^{3}}} \\
& {[t]=s}
\end{aligned}
$$

A partir de esto podemos derivar las siguientes variables adimensionales que serán utilizadas para realizar los cálculos y analizar los resultados

$$
\alpha=\frac{k_{1} \tau}{m \gamma} \quad \Delta k=\frac{k_{2}-k_{1}}{k_{1}} \quad \Delta q=\frac{q_{2}-q_{1}}{q_{1}}
$$

$\alpha$ nos la razón entre dos tiempos característicos del sistema lo cual es muy importante, por un lado tenemos $\frac{k_{1}}{m \gamma}$ con unidades de $\frac{1}{t}$, el tiempo característico del sistema mientras que $\tau$ es el tiempo en el que se realiza el ciclo, tener esta variable nos permite tener una referencia del tamaño del tiempo de ciclo comparado con el tiempo característico del sistema.

$\Delta k$ nos indica qué tan grande es el cambio en la rigidez del potencial comparado con su valor inicial.

$\Delta q$ nos indica qué tan grande es el cambio en la intensidad del ruido comparado con su valor inicial.

La variable adimensional $\alpha$ la podemos determinar a partir de los datos experimentales reportados en el artículo de Martínez et al. $!^{21}$ El tiempo característico está dado por $\frac{k_{1}}{m \gamma}$ en donde $\gamma$ la definimos como

$$
\gamma=\frac{\alpha}{m}
$$

Si suponemos que la partícula de estudio es una esfera, podemos usar la relación que encontró stokes para $\alpha$.

$$
\alpha=6 \pi R \eta
$$

Donde $R$ es el radio de la partícula y $\eta$ es la viscosidad del fluido. Entonces debemos encontrar

$$
\frac{k_{1}}{m \gamma}=\frac{k_{1}}{\alpha}=\frac{k_{1}}{6 \pi R \eta}
$$

Si se encuentra en agua, su viscosidad está dada por

$$
\eta=1.0020 c p=1.0020 \times 10^{-3} \frac{K g}{m s}
$$

Según los datos del experimento realizado por Martínez

$$
r=500 n m=500 \times 10^{-9} m
$$




$$
k_{1}=5.0 \frac{P N}{\mu m}=5.0 \times 10^{-6} \frac{K g}{s^{2}}
$$

Entonces

$$
\frac{k_{1}}{6 \pi R \eta}=\frac{5.0 \times 10^{-6} \frac{\mathrm{Kg}}{\mathrm{s}^{2}}}{6 \pi\left(500 \times 10^{-9} \mathrm{~m}\right)\left(1.0020 \times 10^{-3} \frac{\mathrm{Kg}}{\mathrm{ms}}\right)}=\frac{5.0 \times 10^{-6} \frac{\mathrm{Kg}}{\mathrm{s}^{2}}}{6 \pi\left(5.01 \times 10^{-10}\right) \frac{\mathrm{Kg}}{\mathrm{s}}}=\frac{9980.03992}{6 \pi} \frac{1}{\mathrm{~s}} \simeq 529.456323 \frac{1}{\mathrm{~s}}
$$

y si $\tau=5 s$ entonces obtenemos el valor de

$$
\alpha \simeq 529.456323 \frac{1}{s} \times 5 s=2647.281615
$$

Obtenemos un $\alpha$ muy grande esto implica que el tiempo de realización del ciclo es muy grande comparado con el tiempo característico del problema que es $\frac{1}{529.456323} s$, es decir en este caso es válido el tratamiento en cuasi equilibrio. 


\section{Apéndice B}

\section{Solución a la ecuación de Langevin}

La ecuación de Langevin es la ecuación de movimiento de la partícula browniana al resolverla podemos encontrar la posición de la partícula en un instante dado y su trayectoria que será aleatoria debido al ruido. En nuestros cálculos resolveremos la ecuación para el caso sobre amortiguado

$$
\dot{x}=-\frac{k\left(t^{\prime}\right)}{m \gamma} x\left(t^{\prime}\right)+\frac{\Gamma\left(t^{\prime}\right)}{\gamma}
$$

Es una ecuación lineal diferencial parcial de primer orden con una forma general.

$$
\frac{d x}{d t^{\prime}}=-A\left(t^{\prime}\right) x+B\left(t^{\prime}\right) \Rightarrow \frac{d x}{d t^{\prime}}+A\left(t^{\prime}\right) x=B\left(t^{\prime}\right)
$$

En donde $A\left(t^{\prime}\right)=\frac{k\left(t^{\prime}\right)}{\gamma m}$ y $B\left(t^{\prime}\right)=\frac{\Gamma\left(t^{\prime}\right)}{\gamma}$

Para resolver esta ecuación podemos usar el método del factor integrante, en el cual primeramente debemos de identificar el factor integrante

$$
e^{\int_{t_{0}}^{t^{\prime}} A\left(t^{\prime \prime \prime}\right) d t^{\prime \prime \prime}}
$$

al encontrarlo se debe de multiplicar la ecuación por el factor integrante B.3.

$$
\begin{aligned}
e^{\int_{t_{0}}^{t^{\prime}} A\left(t^{\prime \prime \prime}\right) d t^{\prime \prime \prime}} \frac{d x}{d t^{\prime}}+A\left(t^{\prime}\right) x e^{\int_{t_{0}}^{t^{\prime}} A\left(t^{\prime \prime \prime}\right) d t^{\prime \prime \prime}} & =B\left(t^{\prime}\right) e^{\int_{t_{0}}^{t^{\prime}} A\left(t^{\prime \prime \prime}\right) d t^{\prime \prime \prime}} \\
\frac{d}{d t^{\prime}}\left(x e^{\int_{t_{0}}^{t^{\prime}} A\left(t^{\prime \prime \prime}\right) d t^{\prime \prime \prime}}\right) & =B\left(t^{\prime}\right) e^{\int_{t_{0}}^{t^{\prime}} A\left(t^{\prime \prime \prime}\right) d t^{\prime \prime \prime}} \\
d\left(x\left(t^{\prime}\right) e^{\int_{t_{0}}^{t^{\prime}} A\left(t^{\prime \prime \prime}\right) d t^{\prime \prime \prime}}\right) & =B\left(t^{\prime}\right) e^{\int_{t_{0}}^{t^{\prime}} A\left(t^{\prime \prime \prime}\right) d t^{\prime \prime \prime}} d t^{\prime}
\end{aligned}
$$

Realizando la integración se obtiene

$$
\begin{aligned}
\left.\left(x\left(t^{\prime}\right) e^{\int_{t_{0}}^{t^{\prime}} A\left(t^{\prime \prime \prime}\right) d t^{\prime \prime \prime}}\right)\right|_{t_{0}} ^{t} & =\int_{t_{0}}^{t} B\left(t^{\prime}\right) e^{\int_{t_{0}}^{t^{\prime}} A\left(t^{\prime \prime \prime}\right) d t^{\prime \prime \prime}} d t^{\prime} \\
x(t) e^{\int_{t_{0}}^{t} A\left(t^{\prime \prime \prime}\right) d t^{\prime \prime \prime}}-x\left(t_{0}\right) e^{\int_{t_{0}}^{t_{0}} A\left(t^{\prime \prime \prime}\right) d t^{\prime \prime \prime}} & =\int_{t_{0}}^{t} B\left(t^{\prime}\right) e^{\int_{t_{0}}^{t^{\prime}} A\left(t^{\prime \prime \prime}\right) d t^{\prime \prime \prime}} d t^{\prime}
\end{aligned}
$$

La exponencial del segundo término del lado izquierdo de la ecuación se elimina debido a los límites

$$
\begin{aligned}
x(t) e^{\int_{t_{0}}^{t} A\left(t^{\prime \prime \prime}\right) d t^{\prime \prime \prime}}-x\left(t_{0}\right) & =\int_{t_{0}}^{t} B\left(t^{\prime}\right) e^{\int_{t_{0}}^{t^{\prime}} A\left(t^{\prime \prime \prime}\right) d t^{\prime \prime \prime}} d t^{\prime} \\
x(t) e^{\int_{t_{0}}^{t} A\left(t^{\prime \prime \prime}\right) d t^{\prime \prime \prime}} & =x\left(t_{0}\right)+\int_{t_{0}}^{t} B\left(t^{\prime}\right) e^{\int_{t_{0}}^{t^{\prime}} A\left(t^{\prime \prime \prime}\right) d t^{\prime \prime \prime}} d t^{\prime} \\
x(t) & =x\left(t_{0}\right) e^{-\int_{t_{0}}^{t} A\left(t^{\prime \prime \prime}\right) d t^{\prime \prime \prime}}+e^{-\int_{t_{0}}^{t} A\left(t^{\prime \prime \prime}\right) d t^{\prime \prime \prime}} \int_{t_{0}}^{t} B\left(t^{\prime}\right) e^{\int_{t_{0}}^{t^{\prime}} A\left(t^{\prime \prime \prime}\right) d t^{\prime \prime \prime}} d t^{\prime}
\end{aligned}
$$




$$
\begin{aligned}
& x(t)=x\left(t_{0}\right) e^{-\int_{t_{0}}^{t} A\left(t^{\prime \prime \prime}\right) d t^{\prime \prime \prime}}+\int_{t_{0}}^{t} B\left(t^{\prime}\right) e^{-\int_{t_{0}}^{t} A\left(t^{\prime \prime \prime}\right) d t^{\prime \prime \prime}} e^{\int_{t_{0}}^{t^{\prime}} A\left(t^{\prime \prime \prime}\right) d t^{\prime \prime \prime}} d t^{\prime} \\
& x(t)=x\left(t_{0}\right) e^{-\int_{t_{0}}^{t} A\left(t^{\prime \prime \prime}\right) d t^{\prime \prime \prime}}+\int_{t_{0}}^{t} B\left(t^{\prime}\right) e^{-\int_{t^{\prime}}^{t} A\left(t^{\prime \prime \prime}\right) d t^{\prime \prime \prime}} d t^{\prime}
\end{aligned}
$$

Sustituyendo los valores para $A\left(t^{\prime}\right)$ y $B\left(t^{\prime}\right)$ llegamos a la solución final

$$
x(t)=x\left(t_{0}\right) e^{-\frac{1}{\gamma m} \int_{t_{0}}^{t} k\left(t^{\prime \prime \prime}\right) d t^{\prime \prime \prime}}+\frac{1}{\gamma} \int_{t_{0}}^{t} \Gamma\left(t^{\prime}\right) e^{-\frac{1}{\gamma m} \int_{t^{\prime}}^{t} k\left(t^{\prime \prime \prime}\right) d t^{\prime \prime \prime}} d t^{\prime}
$$

A partir de esta solución, tomando en cuenta una condición inicial para la posición y los protocolos con los que cambia $k\left(t^{\prime \prime \prime}\right)$ y $\Gamma\left(t^{\prime}\right)$ se puede encontrar la posición de la partícula Browniana a un tiempo $t$. Acontinuación se pueden ver las gráficas de la trayectoria de esta partícula en cada uno de los procesos del ciclo de Stirling. Se realizaron tomando en cuenta las variables adimensionales $\alpha=\frac{k 1 \tau}{m \gamma}, \Delta k=\frac{k_{2}-k_{1}}{k_{1}}$ y $\Delta q=\frac{q_{2}-q_{1}}{q_{1}}$

De esta ecuación podemos sacar el promedio y la varianza tomando en cuenta que el ruido del sistema es Gaussiano y debe de cumplir

$$
\begin{gathered}
\langle\Gamma(t)\rangle=0 \\
\left\langle\Gamma(t) \Gamma\left(t^{\prime}\right)\right\rangle=q\left(t^{\prime}\right) \delta\left(t-t^{\prime}\right)
\end{gathered}
$$

El promedio

$$
\begin{aligned}
\langle x(t)\rangle & =x\left(t_{0}\right) e^{-\frac{1}{\gamma m} \int_{t_{0}}^{t} k\left(t^{\prime \prime \prime}\right) d t^{\prime \prime \prime}}+\frac{1}{\gamma} \int_{t_{0}}^{t}\left\langle\Gamma\left(t^{\prime}\right)\right\rangle e^{-\frac{1}{\gamma m} \int_{t^{\prime}}^{t} k\left(t^{\prime \prime \prime}\right) d t^{\prime \prime \prime}} d t^{\prime} \\
\langle x(t)\rangle & =x\left(t_{0}\right) e^{-\frac{1}{\gamma m} \int_{t_{0}}^{t} k\left(t^{\prime \prime \prime}\right) d t^{\prime \prime \prime}}
\end{aligned}
$$

y la función de correlación

$$
\left\langle\left(x\left(t_{1}\right)-\left\langle x\left(t_{1}\right)\right\rangle\right)\left(x\left(t_{2}\right)-\left\langle x\left(t_{2}\right)\right\rangle\right)\right\rangle
$$

En donde

$$
x\left(t_{1}\right)-\left\langle x\left(t_{1}\right)\right\rangle=\frac{1}{\gamma} \int_{t_{0}}^{t_{1}} \Gamma\left(t^{\prime}\right) e^{-\frac{1}{\gamma m} \int_{t^{\prime}}^{t_{1}} k\left(t^{\prime \prime \prime}\right) d t^{\prime \prime \prime}} d t^{\prime}
$$

Entonces

$$
\begin{aligned}
& \left(x\left(t_{1}\right)-\left\langle x\left(t_{1}\right)\right\rangle\right)\left(x\left(t_{2}\right)-\left\langle x\left(t_{2}\right)\right\rangle\right) \\
& =\left(\frac{1}{\gamma} \int_{t_{0}}^{t_{1}} \Gamma\left(t^{\prime}\right) e^{-\frac{1}{\gamma m} \int_{t^{\prime}}^{t_{1}} k\left(t^{\prime \prime \prime}\right) d t^{\prime \prime \prime}} d t^{\prime}\right)\left(\frac{1}{\gamma} \int_{t_{0}}^{t_{2}} \Gamma\left(t^{\prime \prime}\right) e^{-\frac{1}{\gamma m} \int_{t^{\prime \prime}}^{t_{2}} k\left(t^{\prime \prime \prime}\right) d t^{\prime \prime \prime}} d t^{\prime \prime}\right)
\end{aligned}
$$

$\mathrm{Al}$ sacar el promedio

$$
\begin{aligned}
& \left\langle\left(x\left(t_{1}\right)-\left\langle x\left(t_{1}\right)\right\rangle\right)\left(x\left(t_{2}\right)-\left\langle x\left(t_{2}\right)\right\rangle\right)\right\rangle \\
& =\left\langle\frac{1}{\gamma} \int_{t_{0}}^{t_{1}} \Gamma\left(t^{\prime}\right) e^{-\frac{1}{\gamma m} \int_{t^{\prime}}^{t_{1}} k\left(t^{\prime \prime \prime}\right) d t^{\prime \prime \prime}} d t^{\prime} \frac{1}{\gamma} \int_{t_{0}}^{t_{2}} \Gamma\left(t^{\prime \prime}\right) e^{-\frac{1}{\gamma m} \int_{t^{\prime \prime}}^{t_{2}} k\left(t^{\prime \prime \prime}\right) d t^{\prime \prime \prime}} d t^{\prime \prime}\right\rangle \\
& =\frac{1}{\gamma^{2}} \int_{t_{0}}^{t_{1}} d t^{\prime} \int_{t_{0}}^{t_{2}} d t^{\prime \prime}\left\langle\Gamma\left(t^{\prime}\right) \Gamma\left(t^{\prime \prime}\right)\right\rangle e^{-\frac{1}{\gamma m} \int_{t^{\prime}}^{t_{1}} k\left(t^{\prime \prime \prime}\right) d t^{\prime \prime \prime}} e^{-\frac{1}{\gamma m} \int_{t^{\prime \prime}}^{t_{2}} k\left(t^{\prime \prime \prime}\right) d t^{\prime \prime \prime}}
\end{aligned}
$$

con la relación $\mathrm{B} .9$ podemos reescribirlo como

$$
=\frac{1}{\gamma^{2}} \int_{t_{0}}^{t_{1}} d t^{\prime} \int_{t_{0}}^{t_{2}} d t^{\prime \prime} q\left(t^{\prime \prime}\right) \delta\left(t^{\prime}-t^{\prime \prime}\right) e^{-\frac{1}{\gamma m} \int_{t^{\prime}}^{t_{1}} k\left(t^{\prime \prime \prime}\right) d t^{\prime \prime \prime}} e^{-\frac{1}{\gamma m} \int_{t^{\prime \prime}}^{t_{2}} k\left(t^{\prime \prime \prime}\right) d t^{\prime \prime \prime}}
$$

Al efectual la integral sobre $t^{\prime \prime}$ debido a la propiedad de la delta el límite será $t_{1}$ o $t_{2}$ dependiendo de cuál sea el menor

$$
=\frac{1}{\gamma^{2}} \int_{t_{0}}^{t_{1} o t_{2}} d t^{\prime}\left(q\left(t^{\prime}\right) e^{-\frac{1}{\gamma m}\left(\int_{t^{\prime}}^{t_{1}} k\left(t^{\prime \prime}\right) d t^{\prime \prime}+\int_{t^{\prime}}^{t_{2}} k\left(t^{\prime \prime}\right) d t^{\prime \prime}\right)}\right)
$$

Hasta aquí se puede encontrar una solución general sin necesidad de usar el protocolo.

Usamos los protocolos ya adimensionalizados para encontrar el promedio y la correlación. 


\section{Parte 1 del ciclo}

En este proceso el tiempo $(t)$ solo puede tomar valores entre 0 y $\tau / 4$

$$
\begin{aligned}
& k(t)=\frac{k_{1}-k_{2}}{\tau} 4 t+k_{2}=k_{1}\left(1+\Delta k-\frac{4 t \Delta k}{\tau}\right) \\
& q(t)=q_{2}=q_{1}(1+\Delta q)
\end{aligned}
$$

El promedio

$$
\begin{aligned}
\langle x(t)\rangle & =x\left(t_{0}\right) e^{-\frac{1}{\gamma m} \int_{t_{0}}^{t} k\left(t^{\prime \prime \prime}\right) d t^{\prime \prime \prime}}=x\left(t_{0}\right) e^{-\frac{k_{1}}{\gamma m} \int_{0}^{t}\left(1+\Delta k-\frac{4 t^{\prime \prime \prime} \Delta k}{\tau}\right) d t^{\prime \prime \prime}} \\
& =x\left(t_{0}\right) e^{-\left.\frac{k_{1}}{\gamma m}\left[(1+\Delta k) t^{\prime \prime \prime}-\frac{4 t^{\prime \prime \prime 2} \Delta k}{2 \tau}\right]\right|_{0} ^{t}}=x\left(t_{0}\right) e^{-\frac{k_{1}}{\gamma m}\left[(1+\Delta k) t-\frac{4 t^{2} \Delta k}{2 \tau}\right]}
\end{aligned}
$$

La correlación

$$
\begin{aligned}
\left\langle\left(x\left(t_{1}\right)-\left\langle x\left(t_{1}\right)\right\rangle\right)\left(x\left(t_{2}\right)-\left\langle x\left(t_{2}\right)\right\rangle\right)\right\rangle & =\frac{q_{1}(1+\Delta q)}{\gamma^{2}} \int_{t_{0}}^{t_{1} o t_{2}} d t^{\prime} e^{-\frac{k_{1}}{\gamma m}\left(\int_{t^{\prime}}^{t 1}\left(1+\Delta k-\frac{4 t^{\prime \prime} \Delta k}{\tau}\right) d t^{\prime \prime}+\int_{t^{\prime}}^{t_{2}}\left(1+\Delta k-\frac{4 t^{\prime \prime} \Delta k}{\tau}\right) d t^{\prime \prime}\right)} \\
& =\frac{q_{1}(1+\Delta q)}{\gamma^{2}} e^{-\frac{k_{1}}{\gamma m}\left[(1+\Delta k)\left(t_{1}+t_{2}\right)-\frac{4 \Delta k\left(t_{1}^{2}+t_{2}^{2}\right)}{2 \tau}\right]} \int_{t_{0}}^{t_{1} o t_{2}} d t^{\prime} e^{\frac{2 k_{1}}{\gamma m}\left[(1+\Delta k) t^{\prime}-\frac{4 t^{\prime 2} \Delta k}{2 \tau}\right]}
\end{aligned}
$$

Se debe de completar el cuadrado perfecto en la exponencial, al hacerlo la solución de la integral será una función de error debido a que la integral no se hace sobre todo el tempo. Además se tendrán dos soluciones diferentes, estas soluciones dependen del tamaño de $t_{1}$ y $t_{2}$

Cuando $t_{1}>t_{2}$ el límite de la integral es $t_{2}$

$$
\begin{aligned}
\left\langle\left(x\left(t_{1}\right)-\left\langle x\left(t_{1}\right)\right\rangle\right)\left(x\left(t_{2}\right)-\left\langle x\left(t_{2}\right)\right\rangle\right)\right\rangle & =\frac{q_{1}(1+\Delta q)}{\gamma^{2}} \int_{t_{0}}^{t_{1} o t_{2}} d t^{\prime} e^{-\frac{k_{1}}{\gamma m}\left(\int_{t^{\prime}}^{t 1}\left(1+\Delta k-\frac{4 t^{\prime \prime} \Delta k}{\tau}\right) d t^{\prime \prime}+\int_{t^{\prime}}^{t_{2}}\left(1+\Delta k-\frac{4 t^{\prime \prime} \Delta k}{\tau}\right) d t^{\prime \prime}\right)} \\
& =\frac{q_{1}(1+\Delta q)}{\gamma^{2}} e^{-\frac{k_{1}}{\gamma m}\left[(1+\Delta k)\left(t_{1}+t_{2}\right)-\frac{4 \Delta k\left(t_{1}^{2}+t_{2}^{2}\right)}{2 \tau}\right]} \sqrt{\frac{m \pi \gamma \tau}{k_{1} \Delta k}} e^{\frac{k_{1}(1+\Delta k)^{2} \tau}{4 m \gamma k}} \\
& \left(\operatorname{Erf}\left[\frac{\sqrt{k_{1} \tau}(1+\Delta k)}{2 \sqrt{\gamma m \Delta k}}\right]-\operatorname{Erf}\left[\frac{\sqrt{k_{1}}\left(-4 t_{2} \Delta k+\tau+\Delta k \tau\right)}{2 \sqrt{m \gamma \Delta k \tau}}\right]\right)
\end{aligned}
$$

Cuando $t_{2}>t_{1}$ el límite de la integral es $t_{1}$

$$
\begin{aligned}
\left\langle\left(x\left(t_{1}\right)-\left\langle x\left(t_{1}\right)\right\rangle\right)\left(x\left(t_{2}\right)-\left\langle x\left(t_{2}\right)\right\rangle\right)\right\rangle & =\frac{q_{1}(1+\Delta q)}{\gamma^{2}} \int_{t_{0}}^{t_{1} o t_{2}} d t^{\prime} e^{-\frac{k_{1}}{\gamma m}\left(\int_{t^{\prime}}^{t 1}\left(1+\Delta k-\frac{4 t^{\prime \prime} \Delta k}{\tau}\right) d t^{\prime \prime}+\int_{t^{\prime}}^{t_{2}}\left(1+\Delta k-\frac{4 t^{\prime \prime} \Delta k}{\tau}\right) d t^{\prime \prime}\right)} \\
& =\frac{q_{1}(1+\Delta q)}{\gamma^{2}} e^{-\frac{k_{1}}{\gamma m}\left[(1+\Delta k)\left(t_{1}+t_{2}\right)-\frac{4 \Delta k\left(t_{1}^{2}+t_{2}^{2}\right)}{2 \tau}\right]} \sqrt{\frac{m \pi \gamma \tau}{k_{1} \Delta k}} e^{\frac{k_{1}(1+\Delta k)^{2} \tau}{4 m \gamma \Delta k}} \\
& \left(\operatorname{Erf}\left[\frac{\sqrt{k_{1} \tau}(1+\Delta k)}{2 \sqrt{\gamma m \Delta k}}\right]-\operatorname{Erf}\left[\frac{\sqrt{k_{1}}\left(-4 t_{1} \Delta k+\tau+\Delta k \tau\right)}{2 \sqrt{m \gamma \Delta k \tau}}\right]\right)
\end{aligned}
$$

Entonces podemos escribir

$$
\begin{aligned}
\left\langle x\left(t_{1}\right) x\left(t_{2}\right)\right\rangle & =\left\langle x\left(t_{1}\right)\right\rangle\left\langle x\left(t_{2}\right)\right\rangle+\left\langle\left(x\left(t_{1}\right)-\left\langle x\left(t_{1}\right)\right\rangle\right)\left(x\left(t_{2}\right)-\left\langle x\left(t_{2}\right)\right\rangle\right)\right\rangle \\
& =x\left(t_{0}\right)^{2} e^{-\frac{k_{1}}{\gamma m}\left[(1+\Delta k)\left(t_{1}+t_{2}\right)-\frac{4\left(t_{1}^{2}+t_{2}^{2}\right) \Delta k}{2 \tau}\right]}+\frac{q_{1}(1+\Delta q)}{\gamma^{2}} e^{-\frac{k_{1}}{\gamma m}\left[(1+\Delta k)\left(t_{1}+t_{2}\right)-\frac{4 \Delta k\left(t_{1}^{2}+t_{2}^{2}\right)}{2 \tau}\right]} \\
& \sqrt{\frac{m \pi \gamma \tau}{k_{1} \Delta k}} e^{\frac{k_{1}(1+\Delta k)^{2} \tau}{4 m \gamma \Delta k}}\left(\operatorname{Erf}\left[\frac{\sqrt{k_{1} \tau}(1+\Delta k)}{2 \sqrt{\gamma m \Delta k}}\right]-\operatorname{Erf}\left[\left\{\begin{array}{ll}
\frac{\sqrt{k_{1}}\left(-4 t_{2} \Delta k+\tau+\Delta k \tau\right)}{2 \sqrt{m \gamma \Delta k \tau}}, t_{1}>t_{2} & \frac{\sqrt{k_{1}}\left(-4 t_{1} \Delta k+\tau+\Delta k \tau\right)}{2 \sqrt{m \gamma \Delta k \tau}}, t_{2}>t_{1}
\end{array}\right)\right.\right.
\end{aligned}
$$

Cuando $t_{1}=t_{2}$ llegamos al valor que necesitaremos más tarde para encontrar el trabajo.

$$
\begin{aligned}
\left\langle x^{2}(t)\right\rangle & =x\left(t_{0}\right)^{2} e^{-\frac{k_{1}}{\gamma m}\left[(1+\Delta k)(2 t)-\frac{4 t^{2} \Delta k}{\tau}\right]}+\frac{q_{1}(1+\Delta q)}{\gamma^{2}} e^{-\frac{k_{1}}{\gamma m}\left[(1+\Delta k)(2 t)-\frac{4 \Delta k t^{2}}{\tau}\right]} \\
& \sqrt{\frac{m \pi \gamma \tau}{k_{1} \Delta k}} e^{\frac{k_{1}(1+\Delta k)^{2} \tau}{4 m \gamma / k}}\left(\operatorname{Erf}\left[\frac{\sqrt{k_{1} \tau}(1+\Delta k)}{2 \sqrt{\gamma m \Delta k}}\right]-\operatorname{Erf}\left[\frac{\sqrt{k_{1}}(-4 t \Delta k+\tau+\Delta k \tau)}{2 \sqrt{m \gamma \Delta k \tau}}\right]\right)
\end{aligned}
$$




\section{Parte 2 del ciclo}

En este proceso el tiempo $(t)$ solo puede tomar valores entre $\tau / 4$ y $2 \tau / 4$

$$
\begin{aligned}
k(t) & =k_{1} \\
q(t) & =\frac{q_{1}-q_{2}}{\tau} 4\left(t-\frac{\tau}{4}\right)+q_{2}=q_{1}\left(1+\Delta q\left(2-\frac{4 t}{\tau}\right)\right)
\end{aligned}
$$

el promedio

$$
\langle x(t)\rangle=x\left(t_{0}\right) e^{-\frac{1}{\gamma m} \int_{\tau / 4}^{t} k_{1} d t^{\prime \prime \prime}}=x\left(t_{0}\right) e^{-\frac{k_{1}}{\gamma m}(t-\tau / 4)}
$$

La correlación

$$
\begin{aligned}
\left\langle\left(x\left(t_{1}\right)-\left\langle x\left(t_{1}\right)\right\rangle\right)\left(x\left(t_{2}\right)-\left\langle x\left(t_{2}\right)\right\rangle\right)\right\rangle & =\frac{q_{1}}{\gamma^{2}} \int_{\tau / 4}^{t_{1} o t_{2}} d t^{\prime}\left[\left(1+\Delta q\left(2-\frac{4 t}{\tau}\right)\right) e^{-\frac{k_{1}}{\gamma m}\left(\int_{t^{\prime}}^{t 1} d t^{\prime \prime}+\int_{t^{\prime}}^{t_{2}} d t^{\prime \prime}\right)}\right] \\
& =\frac{q_{1}}{\gamma^{2}} \int_{\tau / 4}^{t_{1} o t_{2}} d t^{\prime}\left[\left(1+\Delta q\left(2-\frac{4 t}{\tau}\right)\right) e^{-\frac{k_{1}}{\gamma m}\left(t_{1}+t_{2}-2 t^{\prime}\right)}\right] \\
& =\frac{q_{1}}{\gamma^{2}} e^{-\frac{k_{1}}{\gamma m}\left(t_{1}+t_{2}\right)} \int_{\tau / 4}^{t_{1} o t_{2}} d t^{\prime}\left(1+\Delta q\left(2-\frac{4 t}{\tau}\right)\right) e^{\frac{k_{1}}{\gamma m}\left(2 t^{\prime}\right)} \\
& =\frac{q_{1}}{\gamma^{2}} e^{-\frac{k_{1}}{\gamma m}\left(t_{1}+t_{2}\right)} \int_{\tau / 4}^{t_{1} o t_{2}} d t^{\prime}\left(e^{\frac{k_{1}}{\gamma m}\left(2 t^{\prime}\right)}+\Delta q\left(2-\frac{4 t}{\tau}\right) e^{\frac{k_{1}}{\gamma m}\left(2 t^{\prime}\right)}\right)
\end{aligned}
$$

Hay dos soluciones posibles a esta integral. Cuando $t_{1}>t_{2}$ el límite de la integral es $t_{2}$

$$
\begin{aligned}
& \left\langle\left(x\left(t_{1}\right)-\left\langle x\left(t_{1}\right)\right\rangle\right)\left(x\left(t_{2}\right)-\left\langle x\left(t_{2}\right)\right\rangle\right)\right\rangle \\
& =\frac{q_{1}}{\gamma^{2}} e^{-\frac{k_{1}}{\gamma m}\left(t_{1}+t_{2}\right)}\left[\frac{(1+2 \Delta q)\left(e^{\frac{2 k_{1}}{\gamma m} t_{2}}-e^{\frac{2 k_{1}}{\gamma m} \frac{\tau}{4}}\right)}{\frac{2 k_{1}}{\gamma m}}-\frac{2 \Delta q \gamma m}{k_{1} \tau}\left(-e^{\frac{2 k_{1} t_{2}}{\gamma m}}\left(t_{2}+\frac{\gamma m}{2 k_{1}}\right)+e^{\frac{k_{1} \tau}{2 m \gamma}}\left(\frac{\tau}{4}+\frac{\gamma m}{2 k_{1}}\right)\right)\right] \\
& =\frac{q_{1}}{\gamma^{2}} e^{-\frac{k_{1}}{\gamma m}\left(t_{1}+t_{2}\right)}\left[e^{\frac{2 k_{1} t_{2}}{\gamma m}}\left(\frac{2 \Delta q \gamma m}{k_{1} \tau}\left(t_{2}+\frac{\gamma m}{2 k_{1}}\right)+\frac{1+\Delta q}{\frac{2 k_{1}}{\gamma m}}\right)+e^{\frac{k_{1} \tau}{2 m \gamma}}\left(-\frac{2 \Delta q \gamma m}{k_{1} \tau}\left(\frac{\tau}{4}+\frac{\gamma m}{2 k_{1}}\right)-\frac{1+\Delta q}{\frac{2 k_{1}}{\gamma m}}\right)\right] \\
& =\frac{q_{1}}{\gamma^{2}}\left[e^{-\frac{k_{1}}{\gamma m}\left(t_{1}-t_{2}\right)}\left(\frac{2 \Delta q \gamma m}{k_{1} \tau}\left(t_{2}+\frac{\gamma m}{2 k_{1}}\right)+\frac{1+\Delta q}{\frac{2 k_{1}}{\gamma m}}\right)+e^{-\frac{k_{1}}{\gamma m}\left(t_{1}+t_{2}\right)} e^{\frac{k_{1} \tau}{2 m \gamma}}\left(-\frac{2 \Delta q \gamma m}{k_{1} \tau}\left(\frac{\tau}{4}+\frac{\gamma m}{2 k_{1}}\right)-\frac{1+\Delta q}{\frac{2 k_{1}}{\gamma m}}\right)\right]
\end{aligned}
$$

Cuando $t_{1}<t_{2}$ el límita de la integral es $t_{1}$

$$
\begin{aligned}
& \left\langle\left(x\left(t_{1}\right)-\left\langle x\left(t_{1}\right)\right\rangle\right)\left(x\left(t_{2}\right)-\left\langle x\left(t_{2}\right)\right\rangle\right)\right\rangle \\
& =\frac{q_{1}}{\gamma^{2}} e^{-\frac{k_{1}}{\gamma m}\left(t_{1}+t_{2}\right)}\left[e^{\frac{2 k_{1} t_{1}}{\gamma m}}\left(\frac{2 \Delta q \gamma m}{k_{1} \tau}\left(t_{2}+\frac{\gamma m}{2 k_{1}}\right)+\frac{1+\Delta q}{\frac{2 k_{1}}{\gamma m}}\right)+e^{\frac{k_{1} \tau}{2 m \gamma}}\left(-\frac{2 \Delta q \gamma m}{k_{1} \tau}\left(\frac{\tau}{4}+\frac{\gamma m}{2 k_{1}}\right)-\frac{1+\Delta q}{\frac{2 k_{1}}{\gamma m}}\right)\right] \\
& =\frac{q_{1}}{\gamma^{2}}\left[e^{-\frac{k_{1}}{\gamma m}\left(t_{2}-t_{1}\right)}\left(\frac{2 \Delta q \gamma m}{k_{1} \tau}\left(t_{2}+\frac{\gamma m}{2 k_{1}}\right)+\frac{1+\Delta q}{\frac{2 k_{1}}{\gamma m}}\right)+e^{-\frac{k_{1}}{\gamma m}\left(t_{1}+t_{2}\right)} e^{\frac{k_{1} \tau}{2 m \gamma}}\left(-\frac{2 \Delta q \gamma m}{k_{1} \tau}\left(\frac{\tau}{4}+\frac{\gamma m}{2 k_{1}}\right)-\frac{1+\Delta q}{\frac{2 k_{1}}{\gamma m}}\right)\right]
\end{aligned}
$$

tomando en cuenta los dos casos podemos escribir

$$
\begin{aligned}
& \left\langle\left(x\left(t_{1}\right)-\left\langle x\left(t_{1}\right)\right\rangle\right)\left(x\left(t_{2}\right)-\left\langle x\left(t_{2}\right)\right\rangle\right)\right\rangle \\
& =\frac{q_{1}}{\gamma^{2}}\left[e^{-\frac{k_{1}}{\gamma m}\left|t_{2}-t_{1}\right|}\left(\frac{2 \Delta q \gamma m}{k_{1} \tau}\left(t_{2}+\frac{\gamma m}{2 k_{1}}\right)+\frac{1+\Delta q}{\frac{2 k_{1}}{\gamma m}}\right)+e^{-\frac{k_{1}}{\gamma m}\left(t_{1}+t_{2}\right)} e^{\frac{k_{1} \tau}{2 m \gamma}}\left(-\frac{2 \Delta q \gamma m}{k_{1} \tau}\left(\frac{\tau}{4}+\frac{\gamma m}{2 k_{1}}\right)-\frac{1+\Delta q}{\frac{2 k_{1}}{\gamma m}}\right)\right]
\end{aligned}
$$

Entonces podemos escribir

$$
\begin{aligned}
\left\langle x\left(t_{1}\right) x\left(t_{2}\right)\right\rangle & =\left\langle x\left(t_{1}\right)\right\rangle\left\langle x\left(t_{2}\right)\right\rangle+\left\langle\left(x\left(t_{1}\right)-\left\langle x\left(t_{1}\right)\right\rangle\right)\left(x\left(t_{2}\right)-\left\langle x\left(t_{2}\right)\right\rangle\right)\right\rangle \\
& =x\left(t_{0}\right)^{2} e^{-\frac{k 1}{\gamma m}\left(t_{1}+t_{2}-\frac{\tau}{2}\right)}+\frac{q_{1}}{\gamma^{2}}\left[e^{-\frac{k_{1}}{\gamma m}\left|t_{2}-t_{1}\right|}\left(\frac{2 \Delta q \gamma m}{k_{1} \tau}\left(t_{2}+\frac{\gamma m}{2 k_{1}}\right)+\frac{1+\Delta q}{\frac{2 k_{1}}{\gamma m}}\right)\right. \\
& \left.+e^{-\frac{k_{1}}{\gamma m}\left(t_{1}+t_{2}\right)} e^{\frac{k_{1} \tau}{2 m \gamma}}\left(-\frac{2 \Delta q \gamma m}{k_{1} \tau}\left(\frac{\tau}{4}+\frac{\gamma m}{2 k_{1}}\right)-\frac{1+\Delta q}{\frac{2 k_{1}}{\gamma m}}\right)\right]
\end{aligned}
$$

Cuando $t_{1}=t_{2}$

$\left\langle x^{2}(t)\right\rangle=x\left(t_{0}\right)^{2} e^{-\frac{k_{1}}{\gamma m}\left(2 t-\frac{\tau}{2}\right)}+\frac{q_{1}}{\gamma^{2}}\left[\left(\frac{2 \Delta q \gamma m}{k_{1} \tau}\left(t+\frac{\gamma m}{2 k_{1}}\right)+\frac{1+\Delta q}{\frac{2 k_{1}}{\gamma m}}\right)+e^{-\frac{2 k_{1} t}{\gamma m}} e^{\frac{k_{1} \tau}{2 m \gamma}}\left(-\frac{2 \Delta q \gamma m}{k_{1} \tau}\left(\frac{\tau}{4}+\frac{\gamma m}{2 k_{1}}\right)-\frac{1+\Delta q}{\frac{2 k_{1}}{\gamma m}}\right)\right]$ 


\section{Parte 3 del ciclo}

En este proceso el tiempo $(t)$ solo puede tomar valores entre $2 \tau / 4$ y $3 \tau / 4$ el protocolo es el siguiente

$$
\begin{aligned}
& k(t)=\frac{k_{2}-k_{1}}{\tau} 4\left(t-\frac{2 \tau}{4}\right)+k_{1}=k_{1}\left(1+\Delta k\left(-2+\frac{4 t}{\tau}\right)\right) \\
& q(t)=q_{1}
\end{aligned}
$$

el promedio

$$
\begin{aligned}
\langle x(t)\rangle & =x\left(t_{0}\right) e^{-\frac{1}{\gamma m} \int_{t_{0}}^{t} k\left(t^{\prime \prime \prime}\right) d t^{\prime \prime \prime}} \\
& =x\left(t_{0}\right) e^{-\frac{1}{\gamma m} \int_{2 \tau / 4}^{t}\left(k_{1}\left(1+\Delta k\left(-2+\frac{4 t^{\prime \prime \prime}}{\tau}\right)\right)\right) d t^{\prime \prime \prime}} \\
& =x\left(t_{0}\right) e^{-\frac{k_{1} \tau}{\gamma m}\left[\frac{t}{\tau}-\frac{2 \Delta k t}{\tau}+\frac{2 \Delta k t^{2}}{\tau^{2}}+\frac{1}{2}-\frac{1 \Delta k}{2}\right]}
\end{aligned}
$$

La correlación

$$
\begin{aligned}
\left\langle\left(x\left(t_{1}\right)-\left\langle x\left(t_{1}\right)\right\rangle\right)\left(x\left(t_{2}\right)-\left\langle x\left(t_{2}\right)\right\rangle\right)\right\rangle & =\frac{1}{\gamma^{2}} \int_{t_{0}}^{t_{1} o t_{2}} d t^{\prime}\left(q\left(t^{\prime}\right) e^{-\frac{1}{\gamma m}\left(\int_{t^{\prime}}^{t 1} k\left(t^{\prime \prime}\right) d t^{\prime \prime}+\int_{t^{\prime}}^{t_{2}} k\left(t^{\prime \prime}\right) d t^{\prime \prime}\right)}\right) \\
& =\frac{1}{\gamma^{2}} \int_{t_{0}}^{t_{1} o t_{2}} d t^{\prime} q_{1} e^{-\frac{k_{1}}{\gamma m}\left(\left[(1-2 \Delta k) t^{\prime \prime}+\frac{t^{\prime \prime 2}}{\tau}\right] I_{t^{\prime}}^{t_{1}}+\left[(1-2 \Delta k) t^{\prime \prime}+\frac{t^{\prime \prime 2}}{\tau}\right] I_{t^{\prime}}^{t_{2}}\right)} \\
& =\frac{1}{\gamma^{2}} \int_{t_{0}}^{t_{1} o t_{2}} d t^{\prime} q_{1} e^{-\frac{k_{1}}{\gamma m}\left[\left(t_{1}+t_{2}\right)(1-2 \Delta k)+\frac{t_{1}^{2}+t_{2}^{2}}{\tau}-2\left(t^{\prime}-2 \Delta k t^{\prime}+\frac{t^{\prime 2}}{\tau}\right)\right]} \\
& =\frac{q_{1}}{\gamma^{2}} e^{-\frac{k_{1}}{\gamma m}\left[\left(t_{1}+t_{2}\right)(1-2 \Delta k)+\frac{t_{1}^{2}+t_{2}^{2}}{\tau}\right]} \int_{t_{0}}^{t_{1} o t_{2}} d t^{\prime} e^{\left.\frac{k_{1}}{\gamma m} 2\left(t^{\prime}-2 \Delta k t^{\prime}+\frac{t^{\prime 2}}{\tau}\right)\right]}
\end{aligned}
$$

Se debe de completar el cuadrado perfecto en la exponencialq ue se encuentra dentro de la integral, al hacerlo notamos que la solución a la integral será una función de error.

Cuando $t_{1}>t_{2}$ el límite de la integral es $t_{2}$

$$
\begin{aligned}
& \left\langle\left(x\left(t_{1}\right)-\left\langle x\left(t_{1}\right)\right\rangle\right)\left(x\left(t_{2}\right)-\left\langle x\left(t_{2}\right)\right\rangle\right)\right\rangle \\
& =\frac{q_{1}}{\gamma^{2}} e^{-\frac{k_{1}}{\gamma m}\left[\left(t_{1}+t_{2}\right)(1-2 \Delta k)+\frac{t_{1}^{2}+t_{2}^{2}}{\tau}\right]} \frac{q_{1} \sqrt{\gamma \tau m \pi}}{2 \sqrt{2 k_{1}}}\left[\operatorname{Erfi}\left(\frac{\sqrt{2 k_{1} \tau}(-1+\Delta k)}{\sqrt{m \gamma}}\right)+\operatorname{Erfi}\left(\frac{\sqrt{k_{1}}\left(2 t_{2}+\tau-2 \Delta k \tau\right)}{\sqrt{2 m \gamma \tau}}\right)\right]
\end{aligned}
$$

Cuando $t_{1}<t_{2}$ el límite de la integral es $t_{1}$

$$
\begin{aligned}
& \left\langle\left(x\left(t_{1}\right)-\left\langle x\left(t_{1}\right)\right\rangle\right)\left(x\left(t_{2}\right)-\left\langle x\left(t_{2}\right)\right\rangle\right)\right\rangle \\
& =\frac{q_{1}}{\gamma^{2}} e^{-\frac{k_{1}}{\gamma m}\left[\left(t_{1}+t_{2}\right)(1-2 \Delta k)+\frac{t_{1}^{2}+t_{2}^{2}}{\tau}\right]} \frac{q_{1} \sqrt{\gamma \tau m \pi}}{2 \sqrt{2 k_{1}}}\left[\operatorname{Erfi}\left(\frac{\sqrt{2 k_{1} \tau}(-1+\Delta k)}{\sqrt{m \gamma}}\right)+\operatorname{Erfi}\left(\frac{\sqrt{k_{1}}\left(2 t_{1}+\tau-2 \Delta k \tau\right)}{\sqrt{2 m \gamma \tau}}\right)\right]
\end{aligned}
$$

En general

$$
\begin{aligned}
& \left\langle\left(x\left(t_{1}\right)-\left\langle x\left(t_{1}\right)\right\rangle\right)\left(x\left(t_{2}\right)-\left\langle x\left(t_{2}\right)\right\rangle\right)\right\rangle \\
& =\frac{q_{1}}{\gamma^{2}} e^{-\frac{k_{1}}{\gamma m}\left[\left(t_{1}+t_{2}\right)(1-2 \Delta k)+\frac{t_{1}^{2}+t_{2}^{2}}{\tau}\right]} \frac{q_{1} \sqrt{\gamma \tau m \pi}}{2 \sqrt{2 k_{1}}}\left[\operatorname{Erfi}\left(\frac{\sqrt{2 k_{1} \tau}(-1+\Delta k)}{\sqrt{m \gamma}}\right)+\operatorname{Erfi}\left(\left\{\begin{array}{l}
\frac{\sqrt{k_{1}}\left(2 t_{2}+\tau-2 \Delta k \tau\right)}{\sqrt{2 m \gamma \tau}}, t_{1}>t_{2} \\
\frac{\sqrt{k_{1}}\left(2 t_{1}+\tau-2 \Delta k \tau\right)}{\sqrt{2 m \gamma \tau}}, t_{2}>t_{1}
\end{array}\right)\right]\right.
\end{aligned}
$$

Entonces podemos escribir

$$
\begin{aligned}
\left\langle x\left(t_{1}\right) x\left(t_{2}\right)\right\rangle & =\left\langle x\left(t_{1}\right)\right\rangle\left\langle x\left(t_{2}\right)\right\rangle+\left\langle\left(x\left(t_{1}\right)-\left\langle x\left(t_{1}\right)\right\rangle\right)\left(x\left(t_{2}\right)-\left\langle x\left(t_{2}\right)\right\rangle\right)\right\rangle \\
& =x\left(t_{0}\right)^{2} e^{-\frac{k_{1} \tau}{m \gamma}\left(\frac{t_{1}+t_{2}}{\tau}-\frac{2 \Delta k\left(t_{1}+t_{2}\right)}{\tau}+\frac{\Delta k\left(t_{1}^{2}+t_{2}^{2}\right)}{\tau^{2}}+1-\frac{3 \Delta k}{2}\right)}+\frac{q_{1}}{\gamma^{2}} e^{-\frac{k_{1}}{\gamma m}\left[\left(t_{1}+t_{2}\right)(1-2 \Delta k)+\frac{t_{1}^{2}+t_{2}^{2}}{\tau}\right] \frac{q_{1} \sqrt{\gamma \tau m \pi}}{2 \sqrt{2 k_{1}}} \times} \\
& {\left[\operatorname{Erfi}\left(\frac{\sqrt{2 k_{1} \tau}(-1+\Delta k)}{\sqrt{m \gamma}}\right)+\operatorname{Erfi}\left(\left\{\begin{array}{rr}
\frac{\sqrt{k_{1}}\left(2 t_{2}+\tau-2 \Delta k \tau\right)}{\sqrt{2 m \gamma \tau}}, t_{1}>t_{2} & \\
\frac{\sqrt{k_{1}}\left(2 t_{1}+\tau-2 \Delta k \tau\right)}{\sqrt{2 m \gamma \tau}}, t_{2}>t_{1}
\end{array}\right)\right]\right.}
\end{aligned}
$$

Cuando $t_{1}=t_{2}$ llegamos al valor que necesitaremos más tarde para encontrar el trabajo.

$$
\begin{gathered}
\langle x(t)\rangle=x\left(t_{0}\right)^{2} e^{-\frac{k_{1} \tau}{m \gamma}\left(\frac{2 t}{\tau}-\frac{2 \Delta k(2 t)}{\tau}+\frac{\Delta k\left(2 t^{2}\right)}{\tau^{2}}+1-\frac{3 \Delta k}{2}\right)}+\frac{q_{1}}{\gamma^{2}} e^{-\frac{k_{1}}{\gamma m}\left[(2 t)(1-2 \Delta k)+\frac{2 t^{2}}{\tau}\right]} \frac{q_{1} \sqrt{\gamma \tau m \pi}}{2 \sqrt{2 k_{1}}} \times \\
{\left[\operatorname{Erfi}\left(\frac{\sqrt{2 k_{1} \tau}(-1+\Delta k)}{\sqrt{m \gamma}}\right)+\operatorname{Erfi}\left(\frac{\sqrt{k_{1}}(2 t+\tau-2 \Delta k \tau)}{\sqrt{2 m \gamma \tau}}\right)\right]}
\end{gathered}
$$




\section{Parte 4 del ciclo}

En este proceso el protocolo es el siguiente, el tiempo solo puede tomar valores entre $3 \tau / 4$ y $\tau$

$$
\begin{aligned}
& k(t)=k_{2}=k 1(1+\Delta k) \\
& q(t)=\frac{q_{2}-q_{1}}{\tau} 4\left(t-\frac{3 \tau}{4}\right)+q_{1}=q_{1}\left(1+\Delta q\left(-3+\frac{4 t}{\tau}\right)\right)
\end{aligned}
$$

el promedio

$$
\begin{aligned}
\langle x(t)\rangle & =x\left(t_{0}\right) e^{-\frac{1}{\gamma m} \int_{t_{0}}^{t} k\left(t^{\prime \prime \prime}\right) d t^{\prime \prime \prime}} \\
& =x\left(t_{0}\right) e^{-\frac{(k 1(1+\Delta k))}{\gamma m} \int_{t_{0}}^{t} d t^{\prime \prime \prime}} \\
& =x\left(t_{0}\right) e^{-\frac{(k 1(1+\Delta k))}{\gamma m}\left(t-\frac{3 \tau}{4}\right)}
\end{aligned}
$$

La correlación

$$
\begin{aligned}
\left\langle\left(x\left(t_{1}\right)-\left\langle x\left(t_{1}\right)\right\rangle\right)\left(x\left(t_{2}\right)-\left\langle x\left(t_{2}\right)\right\rangle\right)\right\rangle & \left.=\frac{1}{\gamma^{2}} \int_{t_{0}}^{t_{1} o t_{2}} d t^{\prime} q_{1}\left(1+\Delta q\left(-3+\frac{4 t}{\tau}\right)\right) e^{-\frac{1}{\gamma m}\left(\int_{t^{\prime}}^{t 1} k\left(t^{\prime \prime}\right) d t^{\prime \prime}+\int_{t^{\prime}}^{t_{2}} k\left(t^{\prime \prime}\right) d t^{\prime \prime}\right)}\right) \\
& =\frac{q_{1}}{\gamma^{2}} \int_{t_{0}}^{t_{1} o t_{2}} d t^{\prime}\left(1+\Delta q\left(-3+\frac{4 t}{\tau}\right)\right) e^{-\frac{k_{1}(1+\Delta k)}{\gamma m}\left(t_{1}+t_{2}-2 t^{\prime}\right)} \\
& =\frac{q_{1}}{\gamma^{2}} e^{-\frac{k_{1}(1+\Delta k)}{\gamma m}\left(t_{1}+t_{2}\right)} \int_{t_{0}}^{t_{1} o t_{2}} d t^{\prime}\left(e^{\frac{k_{1}(1+\Delta k)}{\gamma m}\left(2 t^{\prime}\right)}+\Delta q e^{\frac{k_{1}(1+\Delta k)}{\gamma m}\left(2 t^{\prime}\right)}\left(-3+\frac{4 t^{\prime}}{\tau}\right)\right)
\end{aligned}
$$

Hay dos soluciones posibles a esta integral.

Cuando $t_{1}>t_{2}$ el límite de la integral es $t_{2}$

$$
\begin{aligned}
& \left\langle\left(x\left(t_{1}\right)-\left\langle x\left(t_{1}\right)\right\rangle\right)\left(x\left(t_{2}\right)-\left\langle x\left(t_{2}\right)\right\rangle\right)\right\rangle=\frac{q_{1}}{\gamma^{2}} e^{-\frac{k_{1}(1+\Delta k)}{\gamma m}\left(t_{1}+t_{2}\right)} \int_{3 \tau / 4}^{t_{2}} d t^{\prime}\left(e^{\frac{k_{1}(1+\Delta k)}{\gamma m}\left(2 t^{\prime}\right)}(1-3 \Delta q)+\frac{4 t^{\prime} \Delta q}{\tau} e^{\frac{k_{1}(1+\Delta k)}{\gamma m}\left(2 t^{\prime}\right)}\right) \\
& =\frac{q_{1}}{\gamma^{2}} e^{-\frac{k_{1}(1+\Delta k)}{\gamma m}\left(t_{1}+t_{2}\right)}\left[\frac{m \gamma(1-3 \Delta q)\left(e^{\frac{2 t_{2} k_{1}(1+\Delta k)}{\gamma m}}-e^{\frac{3 \tau k_{1}(1+\Delta k)(1-2 \Delta q)}{2 \gamma m}}\right)}{2 k_{1}(1+\Delta k)}\right. \\
& \left.+\frac{4 \gamma m \Delta q}{2 k_{1} \tau(1+\Delta k)}\left(-e^{\frac{2 k_{1}(1+\Delta k) t_{2}}{\gamma m}}\left(t_{2}+\frac{\gamma m}{2 k_{1}(1+\Delta k)}\right)+e^{\frac{3 k_{1}(1+\Delta k) \tau}{2 \gamma m}}\left(\frac{3 \tau}{4}+\frac{\gamma m}{2 k_{1}(1+\Delta k)}\right)\right)\right] \\
& =\frac{m \gamma q_{1}}{2 \gamma^{2} k_{1}(1+\Delta k)} e^{-\frac{k_{1}(1+\Delta k)}{\gamma m}\left(t_{1}+t_{2}\right)}\left[(1-3 \Delta q)\left(e^{\frac{2 t_{2} k_{1}(1+\Delta k)}{\gamma m}}-e^{\frac{3 \tau k_{1}(1+\Delta k)(1-2 \Delta q)}{2 \gamma m}}\right)\right. \\
& \left.+\frac{4 \Delta q}{\tau}\left(-e^{\frac{2 k_{1}(1+\Delta k) t_{2}}{\gamma m}}\left(t_{2}+\frac{\gamma m}{2 k_{1}(1+\Delta k)}\right)+e^{\frac{3 k_{1}(1+\Delta k) \tau}{2 \gamma m}}\left(\frac{3 \tau}{4}+\frac{\gamma m}{2 k_{1}(1+\Delta k)}\right)\right)\right] \\
& =\frac{m \gamma q_{1} e^{-\frac{k_{1}(1+\Delta k)}{\gamma m}\left(t_{1}+t_{2}\right)}}{2 \gamma^{2} k_{1}(1+\Delta k)}\left[e^{\frac{2 t_{2} k_{1}(1+\Delta k)}{\gamma m}}\left(1-3 \Delta q-\frac{4 \Delta q}{\tau}\left(t_{2}+\frac{\gamma m}{2 k_{1}(1+\Delta k)}\right)\right)\right. \\
& \left.+e^{\frac{3 \tau k_{1}(1+\Delta k)}{2 \gamma m}}\left(-1+3 \Delta q+\frac{4 \Delta q}{\tau}\left(\frac{3 \tau}{4}+\frac{\gamma m}{2 k_{1}(1+\Delta k)}\right)\right)\right] \\
& =\frac{m \gamma q_{1}}{2 \gamma^{2} k_{1}(1+\Delta k)}\left[e^{-\frac{k_{1}(1+\Delta k)}{\gamma m}\left(t_{1}+t_{2}\right)} e^{\frac{2 t_{2} k_{1}(1+\Delta k)}{\gamma m}}\left(1-3 \Delta q-\frac{4 \Delta q}{\tau}\left(t_{2}+\frac{\gamma m}{2 k_{1}(1+\Delta k)}\right)\right)\right. \\
& \left.+e^{-\frac{k_{1}(1+\Delta k)}{\gamma m}\left(t_{1}+t_{2}\right)} e^{\frac{3 \tau k_{1}(1+\Delta k)}{2 \gamma m}}\left(-1+3 \Delta q+\frac{4 \Delta q}{\tau}\left(\frac{3 \tau}{4}+\frac{\gamma m}{2 k_{1}(1+\Delta k)}\right)\right)\right] \\
& =\frac{m \gamma q_{1}}{2 \gamma^{2} k_{1}(1+\Delta k)}\left[e^{-\frac{k_{1}(1+\Delta k)}{\gamma m}\left(t_{1}-t_{2}\right)}\left(1-3 \Delta q-\frac{4 \Delta q}{\tau}\left(t_{2}+\frac{\gamma m}{2 k_{1}(1+\Delta k)}\right)\right)\right. \\
& \left.+e^{-\frac{k_{1}(1+\Delta k)}{\gamma m}\left(t_{1}+t_{2}+\frac{3 \tau}{2}\right)}\left(-1+3 \Delta q+\frac{4 \Delta q}{\tau}\left(\frac{3 \tau}{4}+\frac{\gamma m}{2 k_{1}(1+\Delta k)}\right)\right)\right]
\end{aligned}
$$

Cuando $t_{2}>t_{1}$ el límite de la integral es $t_{1}$

$$
\begin{gathered}
\left\langle\left(x\left(t_{1}\right)-\left\langle x\left(t_{1}\right)\right\rangle\right)\left(x\left(t_{2}\right)-\left\langle x\left(t_{2}\right)\right\rangle\right)\right\rangle=\frac{q_{1}}{\gamma^{2}} e^{-\frac{k_{1}(1+\Delta k)}{\gamma m}\left(t_{1}+t_{2}\right)} \int_{3 \tau / 4}^{t_{1}} d t^{\prime}\left(e^{\frac{k_{1}(1+\Delta k)}{\gamma m}\left(2 t^{\prime}\right)}(1-3 \Delta q)+\frac{4 t^{\prime} \Delta q}{\tau} e^{\frac{k_{1}(1+\Delta k)}{\gamma m}\left(2 t^{\prime}\right)}\right) \\
=\frac{m \gamma q_{1}}{2 \gamma^{2} k_{1}(1+\Delta k)}\left[e^{-\frac{k_{1}(1+\Delta k)}{\gamma m}\left(t_{2}-t_{1}\right)}\left(1-3 \Delta q-\frac{4 \Delta q}{\tau}\left(t_{2}+\frac{\gamma m}{2 k_{1}(1+\Delta k)}\right)\right)\right. \\
\left.+e^{-\frac{k_{1}(1+\Delta k)}{\gamma m}\left(t_{1}+t_{2}+\frac{3 \tau}{2}\right)}\left(-1+3 \Delta q+\frac{4 \Delta q}{\tau}\left(\frac{3 \tau}{4}+\frac{\gamma m}{2 k_{1}(1+\Delta k)}\right)\right)\right]
\end{gathered}
$$


Tomando en cuenta los dos casos podemos escribir

$$
\begin{array}{r}
\left\langle\left(x\left(t_{1}\right)-\left\langle x\left(t_{1}\right)\right\rangle\right)\left(x\left(t_{2}\right)-\left\langle x\left(t_{2}\right)\right\rangle\right)\right\rangle=\frac{m \gamma q_{1}}{2 \gamma^{2} k_{1}(1+\Delta k)}\left[e^{-\frac{k_{1}(1+\Delta k)}{\gamma m}\left|t_{2}-t_{1}\right|}\left(1-3 \Delta q-\frac{4 \Delta q}{\tau}\left(t_{2}+\frac{\gamma m}{2 k_{1}(1+\Delta k)}\right)\right)\right. \\
\left.+e^{-\frac{k_{1}(1+\Delta k)}{\gamma m}\left(t_{1}+t_{2}+\frac{3 \tau}{2}\right)}\left(-1+3 \Delta q+\frac{4 \Delta q}{\tau}\left(\frac{3 \tau}{4}+\frac{\gamma m}{2 k_{1}(1+\Delta k)}\right)\right)\right]
\end{array}
$$

Entonces podemos escribir

$$
\begin{aligned}
& \left\langle x\left(t_{1}\right) x\left(t_{2}\right)\right\rangle=\left\langle x\left(t_{1}\right)\right\rangle\left\langle x\left(t_{2}\right)\right\rangle+\left\langle\left(x\left(t_{1}\right)-\left\langle x\left(t_{1}\right)\right\rangle\right)\left(x\left(t_{2}\right)-\left\langle x\left(t_{2}\right)\right\rangle\right)\right\rangle \\
& =x\left(t_{0}\right)^{2} e^{-\frac{k_{1}(1+\Delta k)}{\gamma m}\left(t_{1}+t_{2}-\frac{3 \tau}{2}\right)}+\frac{m \gamma q_{1}}{2 \gamma^{2} k_{1}(1+\Delta k)}\left[e ^ { - \frac { k _ { 1 } ( 1 + \Delta k ) } { \gamma m } | t _ { 2 } - t _ { 1 } | } \left(1-3 \Delta q-\frac{4 \Delta q}{\tau}\left(\left\{\begin{array}{l}
t_{1} \\
t_{2}
\end{array} \quad+\frac{\gamma m}{2 k_{1}(1+\Delta k)}\right)\right)\right.\right. \\
& \left.+e^{-\frac{k_{1}(1+\Delta k)}{\gamma m}\left(t_{1}+t_{2}+\frac{3 \tau}{2}\right)}\left(-1+3 \Delta q+\frac{4 \Delta q}{\tau}\left(\frac{3 \tau}{4}+\frac{\gamma m}{2 k_{1}(1+\Delta k)}\right)\right)\right]
\end{aligned}
$$

Cuando $t_{1}=t_{2}$ llegamos al valor que necesitaremos más tarde para encontrar el trabajo.

$$
\begin{aligned}
& \left\langle x(t)^{2}\right\rangle \\
= & x\left(t_{0}\right)^{2} e^{-\frac{k_{1}(1+\Delta k)}{\gamma m}\left(2 t-\frac{3 \tau}{2}\right)}+\frac{m \gamma q_{1}}{2 \gamma^{2} k_{1}(1+\Delta k)}\left[\left(1-3 \Delta q-\frac{4 \Delta q}{\tau}\left(\frac{\gamma m}{2 k_{1}(1+\Delta k)}\right)\right)\right. \\
+ & \left.e^{-\frac{k_{1}(1+\Delta k)}{\gamma m}\left(2 t+\frac{3 \tau}{2}\right)}\left(-1+3 \Delta q+\frac{4 \Delta q}{\tau}\left(\frac{3 \tau}{4}+\frac{\gamma m}{2 k_{1}(1+\Delta k)}\right)\right)\right]
\end{aligned}
$$





\section{Apéndice $\mathrm{C}$}

\section{Solución a Fokker-Planck}

La solución a Fokker-Planck durante el ciclo nos va a indicar la evolución temporal de la función de distribución/densidad de probabilidad, que nos indica la probabilidad de que la variable $x$ tenga un valor entre $(x, x+d x)$ al tiempo $t$, la cual se propuso con una forma Gaussiana

$$
w(x, t)=\sqrt{\frac{\epsilon(t)}{\pi}} e^{-\epsilon(t) x^{2}}
$$

En esta solución propuesta $\epsilon(t)$ debe de cumplir con

$$
\frac{1}{\epsilon(t)}=\frac{1}{\epsilon\left(t_{0}\right)} e^{-\frac{2}{\gamma m} \int_{t_{0}}^{t} k\left(t^{\prime}\right) d t^{\prime}}+\frac{2}{\gamma^{2}} \int_{t_{0}}^{t} q\left(t^{\prime}\right) e^{-\frac{2}{\gamma m} \int_{t^{\prime}}^{t} k\left(t^{\prime \prime}\right) d t^{\prime \prime}} d t^{\prime}
$$

que se resuelve introduciedo el protocolo del ciclo. La forma de la función de distribución nos indica que la varianza está determinada con $\epsilon(t)$ de la siguiente forma $2 \sigma^{2}=\frac{1}{\epsilon(t)}$, la cual nos indica la variabilidad de nuestros datos respecto a su media. Además se toma en cuenta que el promedio de la posición, por su distribución Gaussiana y porque se encuentra centrada en el origen, es cero.

En este caso el sistema no está en equilibrio y tampoco en un estado estacionario, esto lo podemos notar por la dependencia temporal que se encuentra en la varianza.

\section{C.1. Proceso de 1 a 2}

El protocolo de este proceso implica un aumento en el espacio de movimiento de la partícula Browniana pasando de una rigidez de potencial $k_{2}$ a $k_{1}$ mientras se mantiene una intensidad de ruido contante $q_{2}$ de la siguiente manera

$$
\begin{array}{r}
k(t)=\frac{k_{1}-k_{2}}{\tau} 4 t+k_{2} \\
q(t)=q_{2}
\end{array}
$$

$\mathrm{Al}$ introducir C.3 y C.4 en C.2

tenemos la siguiente ecuación

$$
\frac{1}{\epsilon(t)}=\frac{1}{\epsilon\left(t_{0}\right)} e^{-\frac{2}{\gamma m} \int_{t_{0}}^{t}\left(\frac{k_{1}-k_{2}}{\tau} 4 t^{\prime}+k_{2}\right) d t^{\prime}}+\frac{2}{\gamma^{2}} \int_{t_{0}}^{t} q_{2} e^{-\frac{2}{\gamma m} \int_{t^{\prime}}^{t}\left(\frac{k_{1}-k_{2}}{\tau} 4 t^{\prime \prime}+k_{2}\right) d t^{\prime \prime}} d t^{\prime}
$$

La solución final se reduce en resolver tres integrales

$$
I_{1}=-\frac{2}{\gamma m} \int_{t_{0}}^{t}\left(\frac{k_{1}-k_{2}}{\tau} 4 t^{\prime}+k_{2}\right) d t^{\prime}
$$




$$
\begin{aligned}
& I_{2}=-\frac{2}{\gamma m} \int_{t^{\prime}}^{t}\left(\frac{k_{1}-k_{2}}{\tau} 4 t^{\prime \prime}+k_{2}\right) d t^{\prime \prime} \\
& I_{3}=\int_{t_{0}}^{t} q_{2} e^{I_{2}} d t^{\prime}
\end{aligned}
$$

De esta manera tenemos

$$
\frac{1}{\epsilon(t)}=\frac{1}{\epsilon\left(t_{0}\right)} e^{\left(I_{1}\right)}+\frac{2}{\gamma^{2}} I_{3}
$$

Resolvemos las integrales y las escribimos con las variables adimensionales ya mencionadas $* * \alpha, \Delta k$ y $\Delta q$ y las resolvemos la integral 1

$$
\begin{aligned}
I_{1} & =-\frac{2}{\gamma m} \int_{t_{0}}^{t}\left(\frac{k_{1}-k_{2}}{\tau} 4 t^{\prime}+k_{2}\right) d t^{\prime}=-\left.\frac{2}{\gamma m}\left[\frac{k_{1}-k_{2}}{2 \tau} 4 t^{\prime 2}+k_{2} t^{\prime}\right]\right|_{0} ^{t} \\
& =-\left.\frac{2 k_{1}}{\gamma m}\left[-\frac{\Delta k}{2 \tau} 4 t^{\prime 2}+(\Delta k+1) t^{\prime}\right]\right|_{0} ^{t} \\
& =\frac{2 k_{1} t}{\gamma m \tau}[2 t \Delta k-(\Delta k+1) \tau]
\end{aligned}
$$

La segunda integral es muy parecida, solo cambian los límites.

$$
\begin{aligned}
I_{2} & =\left.\frac{2 k_{1} t}{\gamma m \tau}[2 t \Delta k-(\Delta k+1) \tau]\right|_{t^{\prime}} ^{t} \\
& =\frac{2 k_{1}\left(t-t^{\prime}\right)}{\gamma m \tau}\left[2 t \Delta k+2 t^{\prime} \Delta k-(\Delta k+1) \tau\right] \\
& =\frac{2 \alpha\left(t-t^{\prime}\right)}{\tau^{2}}\left[2 t \Delta k+2 t^{\prime} \Delta k-(\Delta k+1) \tau\right]
\end{aligned}
$$

La última integral se logra sustituyendo en ella la solución de la segunda integral de manera que

$$
\left.I_{3}=\int_{t_{0}}^{t} q_{2} e^{I_{2}} d t^{\prime}=\int_{t_{0}}^{t} q_{2} e^{\frac{2 \alpha\left(t-t^{\prime}\right)}{\tau^{2}}\left[2 t \Delta k+2 t^{\prime} \Delta k-(\Delta k+1) \tau\right.}\right] d t^{\prime}
$$

Para resolver esta integral de una manera más sencilla debemos notar que en la exponencial realmente se encuentra una Gaussiana, lo podemos escribir de la siguiente manera

$$
=\int_{0}^{t} q_{2} e^{\frac{\alpha}{4 \Delta k \tau^{2}}(-4 \Delta k t+(\Delta k+1) \tau)^{2}} e^{-\frac{\alpha}{4 \Delta k \tau^{2}}\left(-4 \Delta k t^{\prime}+(\Delta k+1) \tau\right)^{2}} d t^{\prime}
$$

El primer término no contiene dependencia en la variable de integración por lo que lo podemos sacar de la integral

$$
=q_{2} e^{\frac{\alpha}{4 \Delta k \tau^{2}}(-4 \Delta k t+(\Delta k+1) \tau)^{2}} \int_{0}^{t} e^{-\frac{\alpha}{4 \Delta k \tau^{2}}\left(-4 \Delta k t^{\prime}+(\Delta k+1) \tau\right)^{2}} d t^{\prime}
$$

El término que nos queda adentro de la integral es una Gaussiana, podemos ver más facilmente con un cambio de variable $u=\sqrt{\frac{\alpha}{4 \tau^{2} \Delta k}}\left(-4 \Delta k t^{\prime}+(\Delta k+1) \tau\right)$ por lo que

$$
=\sqrt{\frac{4 \tau^{2} \Delta k}{\alpha}} \frac{q_{2}}{4 \Delta k} e^{\frac{\alpha}{4 \Delta k \tau^{2}}(-4 \Delta k t+(\Delta k+1) \tau)^{2}} \int_{u(t)}^{u(0)} e^{-u^{2}} d u^{\prime}
$$

Al tener la integral de una Gaussiana que no se realiza sobre todo el espacio, entonces surge la función de error la cual

$$
\operatorname{erf}(x)=\frac{1}{\sqrt{\pi}} \int_{-x}^{x} e^{-t^{2}} d t=\frac{2}{\sqrt{\pi}} \int_{0}^{x} e^{-t^{2}} d t
$$

esta función de error puede ser imaginaria cuando la exponencial es imaginaria, en este caso la solución a a integral es

$$
\int_{u(t)}^{u(0)} e^{-u^{2}} d u^{\prime}=\frac{\sqrt{\pi}}{2}(\operatorname{erf}(u(0))-\operatorname{erf}(u(t)))
$$


Regresando a la variable original entonces

$$
=\frac{\sqrt{\pi}}{2}\left(\operatorname{erf}\left[\frac{\sqrt{\alpha}(\Delta k+1)}{2 \sqrt{\Delta k}}\right]-\operatorname{erf}\left[\sqrt{\frac{\alpha}{4 \tau^{2} \Delta k}}(-4 \Delta k t+(\Delta k+1) \tau)\right]\right)
$$

Tomando esto en cuenta podemos encontrar a expresión final de la tercera integral

$$
I_{3}=\frac{\tau q_{2} \sqrt{\pi} e^{\frac{\alpha}{4 \Delta k \tau^{2}}(-4 \Delta k t+(\Delta k+1) \tau)^{2}}}{4 \sqrt{\alpha \Delta k}}\left(\operatorname{erf}\left[\frac{\sqrt{\alpha}(\Delta k+1)}{2 \sqrt{\Delta k}}\right]-\operatorname{erf}\left[\frac{\sqrt{\alpha}(-4 \Delta k t+(\Delta k+1) \tau)}{2 \tau \sqrt{\Delta k}}\right]\right)
$$

Teniendo todas las integrales ya realizadas entonces podemos encontrar la solución

$$
\begin{aligned}
\frac{1}{\epsilon_{1 \rightarrow 2}(t)} & =\frac{1}{\epsilon\left(t_{0}\right)} e^{\left(I_{1}\right)}+\frac{2}{\gamma^{2}} I_{3} \\
& =\frac{e^{\frac{2 \alpha t}{\tau^{2}}[2 t \Delta k-(\Delta k+1) \tau]}}{\epsilon\left(t_{0}\right)} \\
& +\frac{2 \tau q_{2} \sqrt{\pi} e^{\frac{\alpha}{4 \Delta k \tau^{2}}(-4 \Delta k t+(\Delta k+1) \tau)^{2}}}{\gamma^{2} 4 \sqrt{\alpha \Delta k}}\left(\operatorname{erf}\left[\frac{\sqrt{\alpha}(\Delta k+1)}{2 \sqrt{\Delta k}}\right]-\operatorname{erf}\left[\frac{\sqrt{\alpha}(-4 \Delta k t+(\Delta k+1) \tau)}{2 \tau \sqrt{\Delta k}}\right]\right) \\
& =\frac{1}{\epsilon\left(t_{0}\right)}\left(e^{\frac{2 \alpha t}{\tau^{2}}[2 t \Delta k-(\Delta k+1) \tau]}\right. \\
& \left.+\frac{2 \epsilon\left(t_{0}\right) \tau q_{2} \sqrt{\pi} e^{\frac{\alpha}{4 \Delta \tau^{2}}(-4 \Delta k t+(\Delta k+1) \tau)^{2}}}{\gamma^{2} 4 \sqrt{\alpha \Delta k}}\left(\operatorname{erf}\left[\frac{\sqrt{\alpha}(\Delta k+1)}{2 \sqrt{\Delta k}}\right]-\operatorname{erf}\left[\frac{\sqrt{\alpha}(-4 \Delta k t+(\Delta k+1) \tau)}{2 \tau \sqrt{\Delta k}}\right]\right)\right) \\
& =\frac{1}{\epsilon\left(t_{0}\right)}\left(e^{\frac{2 \alpha t}{\tau^{2}}[2 t \Delta k-(\Delta k+1) \tau]}\right. \\
& \left.+\frac{\sqrt{\alpha}(1+\Delta k) \sqrt{\pi} e^{\frac{\alpha}{4 \Delta k \tau^{2}}(-4 \Delta k t+(\Delta k+1) \tau)^{2}}}{2 \sqrt{\alpha}}\left(\operatorname{erf}\left[\frac{\sqrt{\alpha}(\Delta k+1)}{2 \sqrt{\Delta k}}\right]-\operatorname{erf}\left[\frac{\sqrt{\alpha}(-4 \Delta k t+(\Delta k+1) \tau)}{2 \tau \sqrt{\Delta k}}\right]\right)\right) \\
& =\frac{1}{\epsilon\left(t_{0}\right)}\left(e^{\frac{2 \alpha t}{\tau}\left[2 \Delta k \frac{t}{\tau}-(\Delta k+1)\right]}\right. \\
& \left.+\frac{\sqrt{\alpha}(1+\Delta k) \sqrt{\pi} e^{\frac{\alpha}{4 \Delta k}\left(-4 \Delta k \frac{t}{\tau}+(\Delta k+1)\right.}}{2 \sqrt{\Delta k}}\left(\operatorname{erf}\left[\frac{\sqrt{\alpha}(\Delta k+1)}{2 \sqrt{\Delta k}}\right]-\operatorname{erf}\left[\frac{\sqrt{\alpha}\left(-4 \Delta k \frac{t}{\tau}+(\Delta k+1)\right)}{2 \sqrt{\Delta k}}\right]\right)\right)
\end{aligned}
$$

Podemos confirmar el resultado viendo que cuando $t=0, \epsilon(t)$ se reduce al valor de la condición inicial

$$
\begin{aligned}
\frac{1}{\epsilon(t)} & =\frac{1}{\epsilon\left(t_{0}\right)}\left(e^{0}+\frac{\sqrt{\alpha}(1+\Delta k) \sqrt{\pi} e^{\frac{\alpha}{4 \Delta k \tau^{2}}((\Delta k+1) \tau)^{2}}}{2 \sqrt{\Delta k}}\left(\operatorname{erf}\left[\frac{\sqrt{\alpha}(\Delta k+1)}{2 \sqrt{\Delta k}}\right]-\operatorname{erf}\left[\frac{\sqrt{\alpha}((\Delta k+1) \tau)}{2 \tau \sqrt{\Delta k}}\right]\right)\right) \\
& =\frac{1}{\epsilon\left(t_{0}\right)}\left(e^{0}+\frac{\sqrt{\alpha}(1+\Delta k) \sqrt{\pi} e^{\frac{\alpha}{4 \Delta k \tau^{2}}((\Delta k+1) \tau)^{2}}}{2 \sqrt{\Delta k}}(0)\right) \\
& =\frac{1}{\epsilon\left(t_{0}\right)}
\end{aligned}
$$

\section{C.2. Proceso de 2 a 3}

El segundo proceso del cico es la disminución de ruido mientras la rigidez del potencial queda constante

$$
\begin{array}{r}
k(t)=k_{1} \\
q(t)=\frac{\left(q_{1}-q_{2}\right)}{\tau} 4\left(t-\frac{\tau}{4}\right)+q_{2}
\end{array}
$$

$\mathrm{Al}$ introducir el protocolo en $\mathrm{C} .2$ obtenemos

$$
\frac{1}{\epsilon(t)}=\frac{1}{\epsilon\left(t_{0}\right)} e^{-\frac{2}{\gamma m} \int_{t_{0}}^{t} k_{1} d t^{\prime}}+\frac{2}{\gamma^{2}} \int_{t_{0}}^{t}\left(\frac{\left(q_{1}-q_{2}\right)}{\tau} 4\left(t-\frac{\tau}{4}\right)+q_{2}\right) e^{-\frac{2}{\gamma m} \int_{t^{\prime}}^{t} k_{1} d t^{\prime \prime}} d t^{\prime}
$$

Como vimos anteriormente, a solución se reduce a resolver tres integrales

$$
I_{1}=-\frac{2}{\gamma m} \int_{t_{0}}^{t} k_{1} d t^{\prime}
$$




$$
\begin{aligned}
& I_{2}=-\frac{2}{\gamma m} \int_{t^{\prime}}^{t} k_{1} d t^{\prime \prime} \\
& I_{3}=\int_{t_{0}}^{t}\left(\frac{\left(q_{1}-q_{2}\right)}{\tau} 4\left(t^{\prime}-\frac{\tau}{4}\right)+q_{2}\right) e^{I_{2}} d t^{\prime}
\end{aligned}
$$

Las dos primeras integrales son más que triviales, por lo que escribiré diréctamente la solución escrita en sus variables adimensionales.

$$
\begin{aligned}
& I_{1}=-\frac{2 \alpha\left(t-\frac{\tau}{4}\right)}{\tau} \\
& I_{2}=-\frac{2 \alpha\left(t-t^{\prime}\right)}{\tau}
\end{aligned}
$$

Tomando en cuenta esto, entonces nos resta resolver solo una integral.

$$
I_{3}=\int_{t_{0}}^{t}\left(\frac{\left(q_{1}-q_{2}\right)}{\tau} 4\left(t^{\prime}-\frac{\tau}{4}\right)+q_{2}\right) e^{-\frac{2 \alpha\left(t-t^{\prime}\right)}{\tau}} d t^{\prime}
$$

La cual podemos reescribir de la siguiente manera

$$
=\int_{\tau / 4}^{t} \frac{4\left(q_{1}-q_{2}\right) t^{\prime}}{\tau} e^{-\frac{2 \alpha\left(t-t^{\prime}\right)}{\tau}} d t^{\prime}+\int_{t_{0}}^{t}\left(-q_{1}+2 q_{2}\right) e^{-\frac{2 \alpha\left(t-t^{\prime}\right)}{\tau}} d t^{\prime}
$$

La primera integral se resuelve por partes, mientras que la segunda se resuelve directamente con las propiedades de la función exponencial.

$$
=\frac{2\left(q_{1}-q_{2}\right)}{\alpha}\left[t-\frac{\tau}{2 \alpha}-e^{-\frac{2 \alpha}{\tau}(t-\tau / 4)}\left(\frac{\tau}{4}-\frac{\tau}{2 \alpha}\right)\right]+\frac{\left(-q_{1}+2 q_{2}\right) \tau}{2 \alpha}\left[1-e^{-\frac{2 \alpha}{\tau}(t-\tau / 4)}\right]
$$

Desarrollamos para escribirlo de la forma

$$
=\frac{1}{2 \alpha^{2}}\left(4 q_{1} t \alpha-4 q_{2} t \alpha-2 q_{1} \tau+2 q_{2} \tau-q_{1} \alpha \tau+2 q_{2} \alpha \tau+e^{-\frac{2 \alpha}{\tau}(t-\tau / 4)} \tau\left(2 q_{1}-q_{2}(2+\alpha)\right)\right)
$$

Podemos reescribirlo tomando en cuenta las variables adimensionales $\alpha, \Delta q$ y $\Delta k$

$$
=-\frac{q_{1}}{2 \alpha^{2}}\left(4 t \alpha \Delta q+\tau\left(2 \Delta q\left[-1+e^{-\frac{2 \alpha}{\tau}(t-\tau / 4)}\right]+\alpha\left[-1-2 \Delta q+e^{-\frac{2 \alpha}{\tau}(t-\tau / 4)}(1+\Delta q)\right]\right)\right)
$$

Ya tenemos las tres soluciones de las integrales, con esto podemos escribir la solución a Fokker-Planck para el proceso de disminución del ruido a un potencial constante

$$
\begin{aligned}
& \frac{1}{\epsilon_{2 \rightarrow 3}(t)}=-\frac{1}{\epsilon\left(t_{0}\right)} e^{-\frac{2 \alpha\left(t-\frac{\tau}{4}\right)}{\tau}}-\frac{q_{1}}{\gamma^{2} \alpha^{2}}\left(4 t \alpha \Delta q+\tau\left(2 \Delta q\left[-1+e^{-\frac{2 \alpha}{\tau}(t-\tau / 4)}\right]+\alpha\left[-1-2 \Delta q+e^{-\frac{2 \alpha}{\tau}(t-\tau / 4)}(1+\Delta q)\right]\right)\right) \\
& =\frac{1}{\epsilon\left(t_{0}\right)}\left(e^{-\frac{2 \alpha\left(t-\frac{\tau}{4}\right)}{\tau}}-\frac{4 t \alpha \Delta q+\tau\left(2 \Delta q\left[-1+e^{-\frac{2 \alpha}{\tau}(t-\tau / 4)}\right]+\alpha\left[-1-2 \Delta q+e^{-\frac{2 \alpha}{\tau}(t-\tau / 4)}(1+\Delta q)\right]\right)}{\alpha \tau(1+\Delta q)}\right) \\
& =\frac{1}{\epsilon\left(t_{0}\right)}\left(e^{-2 \alpha\left(\frac{t}{\tau}-\frac{1}{4}\right)}-\frac{4 t \alpha \Delta q}{\alpha \tau(1+\Delta q)}-\frac{2 \Delta q\left[-1+e^{-2 \alpha\left(\frac{t}{\tau}-\frac{1}{4}\right)}\right]+\alpha\left[-1-2 \Delta q+e^{-2 \alpha\left(\frac{t}{\tau}-\frac{1}{4}\right)}(1+\Delta q)\right]}{\alpha(1+\Delta q)}\right)
\end{aligned}
$$

Para comprobar que efectivamente la solución tiene sentido, realizamos el análisis en el tiempo inicial del procesot $=\frac{\tau}{4}$, en este caso

$$
\begin{aligned}
\frac{1}{\epsilon(\tau / 4)} & =\frac{1}{\epsilon\left(t_{0}\right)}\left(e^{-\frac{2 \alpha \frac{\tau}{4}(0)}{\tau}}-\frac{4 \frac{\tau}{4} \alpha \Delta q}{\alpha \tau(1+\Delta q)}-\frac{2 \Delta q\left[-1+e^{-\frac{2 \alpha \frac{\tau}{4}}{\tau}(0)}\right]+\alpha\left[-1-2 \Delta q+e^{-\frac{2 \alpha \frac{\tau}{4}}{\tau}(0)}(1+\Delta q)\right]}{\alpha(1+\Delta q)}\right) \\
& =\frac{1}{\epsilon\left(t_{0}\right)}\left(1-\frac{4 \frac{\tau}{4} \alpha \Delta q}{\alpha \tau(1+\Delta q)}-\frac{2 \Delta q[-1+1]+\alpha[-1-2 \Delta q+1(1+\Delta q)]}{\alpha(1+\Delta q)}\right) \\
& =\frac{1}{\epsilon\left(t_{0}\right)}\left(1-\frac{\Delta q-\Delta q}{(1+\Delta q)}\right) \\
& =\frac{1}{\epsilon\left(t_{0}\right)}
\end{aligned}
$$

la solución es la condición inicial del proceso como se esperaba. 


\section{C.3. Proceso de 3 a 4}

El tercer proceso es un aumento en la rigidez del potencial con una intensidad de ruido constante

$$
\begin{aligned}
& k(t)=\frac{k_{2}-k_{1}}{\tau} 4\left(t-\frac{2 \tau}{4}\right)+k_{1} \\
& q(t)=q_{1}
\end{aligned}
$$

La solución de este proceso es

$$
\frac{1}{\epsilon_{3 \rightarrow 4}(t)}=\frac{1}{\epsilon\left(t_{0}\right)} e^{-\frac{2}{\gamma m} \int_{t_{0}}^{t}\left(\frac{k_{2}-k_{1}}{\tau} 4\left(t^{\prime}-\frac{2 \tau}{4}\right)+k_{1}\right) d t^{\prime}}+\frac{2}{\gamma^{2}} \int_{t_{0}}^{t} q_{1} e^{-\frac{2}{\gamma m} \int_{t^{\prime}}^{t}\left(\frac{k_{2}-k_{1}}{\tau} 4\left(t^{\prime \prime}-\frac{2 \tau}{4}\right)+k_{1}\right) d t^{\prime \prime}} d t^{\prime}
$$

Que como en los procesos anteriores se reduce a resolver las tres integrales

$$
\begin{aligned}
I_{1} & =-\frac{2}{\gamma m} \int_{t_{0}}^{t}\left(\frac{k_{2}-k_{1}}{\tau} 4\left(t^{\prime}-\frac{2 \tau}{4}\right)+k_{1}\right) d t^{\prime} \\
I_{2} & =-\frac{2}{\gamma m} \int_{t^{\prime}}^{t}\left(\frac{k_{2}-k_{1}}{\tau} 4\left(t^{\prime \prime}-\frac{2 \tau}{4}\right)+k_{1}\right) d t^{\prime \prime} \\
I_{3} & =\int_{t_{0}}^{t} q_{1} e^{I_{2}} d t^{\prime}
\end{aligned}
$$

Entonces

$$
\frac{1}{\epsilon(t)}=\frac{1}{\epsilon\left(t_{0}\right)} e^{I_{1}}+\frac{2}{\gamma^{2}} I_{3}
$$

Las primeras dos integrales son muy sencillas, se resuelven de manera muy parecida que en el proceso uno, escribiré únicamente los resultados en su forma adimensional

$$
\begin{aligned}
& I_{1}=-\frac{\alpha(2 t-\tau)(2 t \Delta k+\tau-\Delta k \tau)}{\tau^{2}} \\
& I_{2}=-\frac{2 \alpha\left(t-t^{\prime}\right)\left(2 t \Delta k+2 t^{\prime} \Delta k+\tau-2 \Delta k \tau\right)}{\tau^{2}}
\end{aligned}
$$

Para resolver la tercera integral, podemos reescribir $I_{2}$ de la siguiente manera

$$
=-\frac{\alpha}{4 \Delta k \tau^{2}}\left[(4 \Delta k t+2 \Delta k \tau-\tau)^{2}-\left(4 \Delta k t^{\prime}-2 \Delta k \tau+\tau\right)^{2}\right]
$$

Tomando esto en cuenta entonces tenemos

$$
I_{3}=q_{1} e^{-\frac{\alpha}{4 \Delta k \tau^{2}}(4 \Delta k t-2 \Delta k \tau+\tau)^{2}} \int_{\frac{\tau}{2}}^{t} e^{\frac{\alpha}{4 \Delta k \tau^{2}}\left(4 \Delta k t^{\prime}-2 \Delta k \tau+\tau\right)^{2}} d t^{\prime}
$$

La integral restante es una función de error imaginaria, esta función tiene la siguiente propiedad

$$
\operatorname{erfi}(x)=\frac{2}{\sqrt{\pi}} \int_{0}^{x} e^{t^{2}} d t
$$

Tomando esto en cuenta entonces la solución de $I_{3}$ es la siguiente

$$
=\frac{q 1 \sqrt{\pi} e^{-\frac{\alpha}{4 \Delta k \tau^{2}}(4 \Delta k t-2 \Delta k \tau+\tau)^{2}}}{4 \tau \sqrt{\alpha \Delta k}}\left[\operatorname{erfi}\left(\frac{\sqrt{\alpha}}{2 \sqrt{\Delta k}}\right)-\operatorname{erfi}\left(\frac{\sqrt{\alpha}(4 t \Delta k+\tau-2 \Delta k \tau)}{2 \tau \sqrt{\Delta k}}\right)\right]
$$

Podemos escribir la solución en su forma completa

$$
\begin{aligned}
& \frac{1}{\epsilon_{3 \rightarrow 4}(t)}=\frac{1}{\epsilon\left(t_{0}\right)} e^{-\frac{\alpha(2 t-\tau)(2 t \Delta k+\tau-\Delta k \tau)}{\tau^{2}}}+\frac{2 q 1 \sqrt{\pi} e^{-\frac{\alpha}{4 \Delta k \tau^{2}}(4 \Delta k t-2 \Delta k \tau+\tau)^{2}}}{4 \gamma^{2} \tau \sqrt{\alpha \Delta k}}\left[\operatorname{erfi}\left(\frac{\sqrt{\alpha}}{2 \sqrt{\Delta k}}\right)-\operatorname{erfi}\left(\frac{\sqrt{\alpha}(4 t \Delta k+\tau-2 \Delta k \tau)}{2 \tau \sqrt{\Delta k}}\right)\right] \\
& =\frac{1}{\epsilon\left(t_{0}\right)}\left(e^{-\frac{\alpha(2 t-\tau)(2 t \Delta k+\tau-\Delta k \tau)}{\tau^{2}}}-\frac{\sqrt{\alpha} \sqrt{\pi} e^{-\frac{\alpha}{4 \Delta k \tau^{2}}(4 \Delta k t-2 \Delta k \tau+\tau)^{2}}}{2 \sqrt{\Delta k}}\left[\operatorname{erfi}\left(\frac{\sqrt{\alpha}}{2 \sqrt{\Delta k}}\right)-\operatorname{erfi}\left(\frac{\sqrt{\alpha}(4 t \Delta k+\tau-2 \Delta k \tau)}{2 \tau \sqrt{\Delta k}}\right)\right]\right)
\end{aligned}
$$


$=\frac{1}{\epsilon\left(t_{0}\right)}\left(e^{-\alpha\left(2 \frac{t}{\tau}-1\right)\left(2 \Delta k \frac{t}{\tau}+1-\Delta k\right)}-\frac{\sqrt{\alpha} \sqrt{\pi} e^{-\frac{\alpha}{4 \Delta k}\left(4 \Delta k \frac{t}{\tau}-2 \Delta k+1\right)^{2}}}{2 \sqrt{\Delta k}}\left[\operatorname{erfi}\left(\frac{\sqrt{\alpha}}{2 \sqrt{\Delta k}}\right)-\operatorname{erfi}\left(\frac{\sqrt{\alpha}\left(4 \Delta k \frac{t}{\tau}+1-2 \Delta k\right)}{2 \sqrt{\Delta k}}\right)\right]\right)$

para confirmar la solución, la analizamos cuando $t=\tau / 2$

$$
\begin{aligned}
& =\frac{1}{\epsilon\left(t_{0}\right)}\left(e^{-\frac{\alpha(\tau / 2)^{2}\left(2-\frac{\tau}{\tau / 2}\right)\left(2 \Delta k+\frac{\tau}{\tau / 2}-\Delta k \frac{\tau}{\tau / 2}\right)}{\tau^{2}}}-\frac{\sqrt{\alpha} \sqrt{\pi} e^{-\frac{\alpha(\tau / 2)^{2}}{4 \Delta k \tau^{2}}\left(4 \Delta k-2 \Delta k \frac{\tau}{\tau / 2}+\frac{\tau}{\tau / 2}\right)^{2}}}{2 \sqrt{\Delta k}}\left[\operatorname{erfi}\left(\frac{\sqrt{\alpha}}{2 \sqrt{\Delta k}}\right)\right.\right. \\
& \left.\left.-\operatorname{erfi}\left(\frac{\sqrt{\alpha} \tau / 2\left(4 \Delta k+\frac{\tau}{\tau / 2}-2 \Delta k \frac{\tau}{\tau / 2}\right)}{2 \tau \sqrt{\Delta k}}\right)\right]\right) \\
& =\frac{1}{\epsilon\left(t_{0}\right)}\left(e^{-\frac{\alpha(\tau / 2)^{2}(0)(2 \Delta k+2-2 \Delta k)}{\tau^{2}}}-\frac{\sqrt{\alpha} \sqrt{\pi} e^{-\frac{\alpha(\tau / 2)^{2}}{4 \Delta k \tau^{2}}(4 \Delta k-4 \Delta k+2)^{2}}}{2 \sqrt{\Delta k}}\left[\operatorname{erfi}\left(\frac{\sqrt{\alpha}}{2 \sqrt{\Delta k}}\right)\right.\right. \\
& \left.\left.-\operatorname{erfi}\left(\frac{\sqrt{\alpha} \tau / 2(4 \Delta k+2-4 \Delta k)}{2 \tau \sqrt{\Delta k}}\right)\right]\right) \\
& =\frac{1}{\epsilon\left(t_{0}\right)}\left(e^{-\frac{\alpha(\tau / 2)^{2}(0)(2 \Delta k+2-2 \Delta k)}{\tau^{2}}}-\frac{\sqrt{\alpha} \sqrt{\pi} e^{-\frac{\alpha(\tau / 2)^{2}}{4 \Delta k \tau^{2}} 4}}{2 \sqrt{\Delta k}}\left[\operatorname{erfi}\left(\frac{\sqrt{\alpha}}{2 \sqrt{\Delta k}}\right)-\operatorname{erfi}\left(\frac{\sqrt{\alpha} \tau / 2(2)}{2 \tau \sqrt{\Delta k}}\right)\right]\right) \\
& =\frac{1}{\epsilon\left(t_{0}\right)}\left(e^{0}-\frac{\sqrt{\alpha} \sqrt{\pi} e^{-\frac{\alpha(\tau / 2)^{2}}{4 \Delta k \tau^{2}} 4}}{2 \sqrt{\Delta k}}\left[\operatorname{erfi}\left(\frac{\sqrt{\alpha}}{2 \sqrt{\Delta k}}\right)-\operatorname{erfi}\left(\frac{\sqrt{\alpha}}{2 \sqrt{\Delta k}}\right)\right]\right) \\
& =\frac{1}{\epsilon\left(t_{0}\right)}\left(1-\frac{\left.\sqrt{\alpha} \sqrt{\pi} e^{-\frac{\alpha}{4 \Delta k}}(0)\right)}{2 \sqrt{\Delta k}}\right. \\
& =\frac{1}{\epsilon\left(t_{0}\right)}
\end{aligned}
$$

en este caso la solución se reduce a la condición inicial

\section{C.4. Proceso de 4 a 1}

El último proceso que cierra el ciclo es un aumento en la intensidad del ruido mientras se mantiene la rigidez del potencial de atrapamiento constante.

$$
\begin{aligned}
& k(t)=k_{2} \\
& q(t)=\frac{q_{2}-q_{1}}{\tau} 4\left(t-\frac{3 \tau}{4}\right)+q_{1}
\end{aligned}
$$

La solución de este proceso

$$
\frac{1}{\epsilon_{4 \rightarrow 1}(t)}=\frac{1}{\epsilon\left(t_{0}\right)} e^{-\frac{2}{\gamma m} \int_{t_{0}}^{t} k_{2} d t^{\prime}}+\frac{2}{\gamma^{2}} \int_{t_{0}}^{t}\left(\frac{q_{2}-q_{1}}{\tau} 4\left(t-\frac{3 \tau}{4}\right)+q_{1}\right) e^{-\frac{2}{\gamma m} \int_{t^{\prime}}^{t} k_{2} d t^{\prime \prime}} d t^{\prime}
$$

La solución la encontraremos resolviento tres integrales, esto llega a ser repetitivo

$$
\begin{aligned}
& I_{1}=-\frac{2}{\gamma m} \int_{t_{0}}^{t} k_{2} d t^{\prime} \\
& I_{2}=-\frac{2}{\gamma m} \int_{t^{\prime}}^{t} k_{2} d t^{\prime \prime} \\
& I_{3}=\int_{t_{0}}^{t}\left(\frac{q_{2}-q_{1}}{\tau} 4\left(t-\frac{3 \tau}{4}\right)+q_{1}\right) e^{I_{2}} d t^{\prime}
\end{aligned}
$$

Las soluciónes de las primeras dos integrales en sus variables adimensionales son

$$
\begin{aligned}
& I_{1}=-\frac{2 \alpha(1+\Delta k)\left(t-\frac{3 \tau}{4}\right)}{\tau} \\
& I_{2}=-\frac{2 \alpha\left(t-t^{\prime}\right)(1+\Delta k)}{\tau}
\end{aligned}
$$


La tercera integral tiene la siguiente forma

$$
I_{3}=\int_{t_{0}}^{t}\left(\frac{q_{2}-q_{1}}{\tau} 4\left(t-\frac{3 \tau}{4}\right)+q_{1}\right) e^{-\frac{2 \alpha\left(t-t^{\prime}\right)(1+\Delta k)}{\tau}} d t^{\prime}
$$

La podemos reescribir en dos integrales

$$
=\int_{t_{0}}^{t} \frac{q_{2}-q_{1}}{\tau} 4 t e^{-\frac{2 \alpha\left(t-t^{\prime}\right)(1+\Delta k)}{\tau}} d t^{\prime}+\int_{t_{0}}^{t}\left(-3 q_{2}+4 q_{1}\right) e^{-\frac{2 \alpha\left(t-t^{\prime}\right)(1+\Delta k)}{\tau}} d t^{\prime}
$$

La primera parte de la expresión se resuelve por integración por partes y la segunda con las propiedades de la exponencial.

$$
=\frac{q_{2}-q_{1}}{2 \alpha^{2}(1+\Delta k)^{2}}\left[4 \alpha t(1+\Delta k)-2 \tau-e^{-\frac{2 \alpha(1+\Delta k)}{\tau}\left(t-\frac{3 \tau}{4}\right)}(3 \tau \alpha(1+\Delta k)-2 \tau)\right]+\frac{\tau\left(-3 q_{2}+4 q_{1}\right)}{2 \alpha(1+\Delta k)}\left[1-e^{\left.-\frac{\alpha(1+\Delta k)\left(t-\frac{3 \tau}{4}\right)}{\tau}\right]}\right]_{\text {(C.81) }}
$$

Reescribiendo la solución de la tercera integral en su forma adimensioal

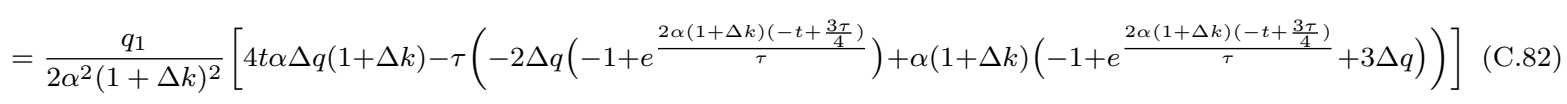

La solución en este proceso es

$$
\begin{aligned}
& \frac{1}{\epsilon_{4 \rightarrow 1}(t)}=\frac{e^{-\frac{2 \alpha(1+\Delta k)\left(t-\frac{3 \tau}{4}\right)}{\tau}}}{\epsilon\left(t_{0}\right)}+ \\
& \frac{q_{1}}{\gamma^{2} \alpha^{2}(1+\Delta k)^{2}}\left[4 t \alpha \Delta q(1+\Delta k)-\tau\left(-2 \Delta q\left(-1+e^{\frac{2 \alpha(1+\Delta k)\left(-t+\frac{3 \tau}{4}\right)}{\tau}}\right)+\alpha(1+\Delta k)\left(-1+e^{\frac{2 \alpha(1+\Delta k)\left(-t+\frac{3 \tau}{4}\right)}{\tau}}+3 \Delta q\right)\right)\right] \\
& =\frac{1}{\epsilon\left(t_{0}\right)}\left[e^{\frac{2 \alpha(1+\Delta k)\left(-t+\frac{3 \tau}{4}\right)}{\tau}}+\right. \\
& \left.\frac{1}{\alpha \tau(1+\Delta k)}\left[4 t \alpha \Delta q(1+\Delta k)-\tau\left(-2 \Delta q\left(-1+e^{\frac{2 \alpha(1+\Delta k)\left(-t+\frac{3 \tau}{4}\right)}{\tau}}\right)+\alpha(1+\Delta k)\left(-1+e^{\frac{2 \alpha(1+\Delta k)\left(-t+\frac{3 \tau}{4}\right)}{\tau}}+3 \Delta q\right)\right)\right]\right] \\
& =\frac{1}{\epsilon\left(t_{0}\right)}\left[e^{2 \alpha(1+\Delta k)\left(-\frac{t}{\tau}+\frac{3}{4}\right)}+\frac{1}{\alpha(1+\Delta k)}\right. \\
& \left.\left[4 \alpha \Delta q \frac{t}{\tau}(1+\Delta k)-\left(-2 \Delta q\left(-1+e^{2 \alpha(1+\Delta k)\left(-\frac{t}{\tau}+\frac{3}{4}\right)}\right)+\alpha(1+\Delta k)\left(-1+e^{2 \alpha(1+\Delta k)\left(-\frac{t}{\tau}+\frac{3}{4}\right)}+3 \Delta q\right)\right)\right]\right]
\end{aligned}
$$

Analizamos la solución en el tiempo inicial del proceso $t=\frac{3 \tau}{4}$

$$
\begin{aligned}
& =\frac{1}{\epsilon\left(t_{0}\right)}\left[e^{\frac{2 \alpha \frac{3 \tau}{4}(1+\Delta k)\left(-1+\frac{3 \tau}{4 \frac{3 \tau}{4}}\right)}{\tau}}+\right. \\
& \left.\frac{\frac{3 \tau}{4}}{\alpha \tau(1+\Delta k)}\left[4 \alpha \Delta q(1+\Delta k)-\frac{\tau}{\frac{3 \tau}{4}}\left(-2 \Delta q\left(-1+e^{\frac{2 \alpha t(1+\Delta k)\left(-1+\frac{3 \tau}{4 \frac{3 \tau}{4}}\right)}{\tau}}\right)+\alpha(1+\Delta k)\left(-1+e^{\frac{2 \alpha \frac{3 \tau}{4}(1+\Delta k)\left(-1+\frac{3 \tau}{4 \frac{3 \tau}{4}}\right)}{\tau}}+3 \Delta q\right)\right)\right]\right] \\
& =\frac{1}{\epsilon\left(t_{0}\right)}\left[e^{\frac{2 \alpha \frac{3 \tau}{4}(1+\Delta k)(-1+1)}{\tau}}+\right. \\
& \left.\frac{3}{4 \alpha \tau(1+\Delta k)}\left[4 \alpha \Delta q(1+\Delta k)-\frac{4}{3}\left(-2 \Delta q\left(-1+e^{\frac{2 \alpha t(1+\Delta k)(-1+1)}{\tau}}\right)+\alpha(1+\Delta k)\left(-1+e^{\frac{2 \alpha \frac{3 \tau}{4}(1+\Delta k)(-1+1)}{\tau}}+3 \Delta q\right)\right)\right]\right] \\
& =\frac{1}{\epsilon\left(t_{0}\right)}\left[e^{0}+\frac{3}{4 \alpha \tau(1+\Delta k)}\left[4 \alpha \Delta q(1+\Delta k)-\frac{4}{3}\left(-2 \Delta q\left(-1+e^{0}\right)+\alpha(1+\Delta k)\left(-1+e^{0}+3 \Delta q\right)\right)\right]\right] \\
& =\frac{1}{\epsilon\left(t_{0}\right)}\left[1+\frac{3}{4 \alpha \tau(1+\Delta k)}\left[4 \alpha \Delta q(1+\Delta k)-\frac{4}{3}(-2 \Delta q(-1+1)+\alpha(1+\Delta k)(-1+1+3 \Delta q))\right]\right] \\
& =\frac{1}{\epsilon\left(t_{0}\right)}\left[1+\frac{3}{4 \alpha \tau(1+\Delta k)}\left[4 \alpha \Delta q(1+\Delta k)-\frac{4}{3}(\alpha(1+\Delta k)(3 \Delta q))\right]\right] \\
& =\frac{1}{\epsilon\left(t_{0}\right)}
\end{aligned}
$$

La solución regresa al valor esperado en el tiempo inicial. 



\section{Apéndice D}

\section{Cálculos del calor}

El calor está dado por la interacción de la partícula con el medio en el que se encuentra, de la ecuación de langevin encontramos que la interacción con el medio está dada por los términos

$$
Q=\left(-\gamma \frac{d x}{d t}+\Gamma(t)\right) \circ d x=\frac{k(t)}{2} d\left(x^{2}\right)
$$

Debido a que el sistema es una partícula Browniana entonces la posición es una variable aleatoria y el proceso es estocástico, la solución de esta ecuación tendrá un valor diferente en cada realización del proceso pero el promedio sobre el ensamble está dado por

$$
\langle\Delta Q\rangle=\int_{t_{0}}^{t_{f}}\left[-\frac{k^{2}(t)}{2 m \gamma \epsilon(t)}+\frac{k(t) q(t)}{2 \gamma^{2}}\right] d t
$$

ya hemos encontrado que $\frac{1}{\epsilon(t)}$ es diferente para cada proceso

\section{D.1. 1 a 2}

El primer proceso se realiza con el protocolo

$$
\begin{aligned}
& k(t)=\frac{k_{1}-k_{2}}{\tau} 4 t+k_{2} \\
& q(t)=q_{2}
\end{aligned}
$$

es un cambio en la rigidez del potencial mientras se mantiene constante el ruido. y la forma de $\epsilon(t)$ está dada por:

$$
\begin{aligned}
\frac{1}{\epsilon_{1 \rightarrow 2}} & =\frac{1}{\epsilon\left(t_{0}\right)}\left(e^{\frac{2 \alpha t^{2}}{\tau^{2}}\left[2 \Delta k-(\Delta k+1) \frac{\tau}{t}\right]}\right. \\
& \left.+\frac{\sqrt{\alpha}(1+\Delta k) \sqrt{\pi} e^{\frac{\alpha t^{2}}{4 \Delta k \tau^{2}}\left(-4 \Delta k+(\Delta k+1) \frac{\tau}{t}\right)^{2}}}{2 \sqrt{\Delta k}}\left(\operatorname{erf}\left[\frac{\sqrt{\alpha}(\Delta k+1)}{2 \sqrt{\Delta k}}\right]-\operatorname{erf}\left[\frac{\sqrt{\alpha} t\left(-4 \Delta k+(\Delta k+1) \frac{\tau}{t}\right)}{2 \tau \sqrt{\Delta k}}\right]\right)\right)
\end{aligned}
$$

En este proceso el promedio del calor se obtiene realizando las siguientes integrales

$$
\langle\Delta Q\rangle=\underbrace{\int_{0}^{\tau / 4}\left[-\frac{k^{2}(t)}{2 m \gamma \epsilon(t)}\right] d t}_{I_{1,1}}+\underbrace{\int_{0}^{\tau / 4}\left[\frac{k(t) q(t)}{2 \gamma^{2}}\right] d t}_{I_{1,2}}
$$

En donde

$$
\begin{aligned}
I_{1,1} & =\int_{0}^{\tau / 4}\left\{( - \frac { ( \frac { k _ { 1 } - k _ { 2 } } { \tau } 4 t + k _ { 2 } ) ^ { 2 } } { 2 m \gamma } ) \frac { 1 } { \epsilon ( t _ { 0 } ) } \left(e^{\frac{2 \alpha t^{2}}{\tau^{2}}\left[2 \Delta k-(\Delta k+1) \frac{\tau}{t}\right]}\right.\right. \\
& \left.\left.+\frac{\sqrt{\alpha}(1+\Delta k) \sqrt{\pi} e^{\frac{\alpha t^{2}}{4 \Delta k \tau^{2}}\left(-4 \Delta k+(\Delta k+1) \frac{\tau}{t}\right)^{2}}}{2 \sqrt{\Delta k}}\left(\operatorname{erf}\left[\frac{\sqrt{\alpha}(\Delta k+1)}{2 \sqrt{\Delta k}}\right]-\operatorname{erf}\left[\frac{\sqrt{\alpha} t\left(-4 \Delta k+(\Delta k+1) \frac{\tau}{t}\right)}{2 \tau \sqrt{\Delta k}}\right]\right)\right)\right\} d t
\end{aligned}
$$




$$
I_{1,2}=\int_{0}^{\tau / 4}\left\{\frac{q_{2}\left(\frac{k_{1}-k_{2}}{\tau} 4 t+k_{2}\right)}{2 \gamma^{2}}\right\} d t
$$

Resolveremos primero D.7 podemos reescribirla de la siguiente manera

$$
\begin{aligned}
I_{1,1} & =-\frac{1}{2 m \gamma \epsilon\left(t_{0}\right)}\left\{\int_{0}^{\tau / 4}\left(\frac{k_{1}-k_{2}}{\tau} 4 t+k_{2}\right)^{2}\left(e^{\frac{2 \alpha t^{2}}{\tau^{2}}\left[2 \Delta k-(\Delta k+1) \frac{\tau}{t}\right]}\right) d t\right. \\
& +\int_{0}^{\tau / 4} \frac{\sqrt{\alpha}(1+\Delta k) \sqrt{\pi}\left(\frac{k_{1}-k_{2}}{\tau} 4 t+k_{2}\right)^{2} e^{\frac{\alpha t^{2}}{4 \Delta k \tau^{2}}\left(-4 \Delta k+(\Delta k+1) \frac{\tau}{t}\right)^{2}}}{2 \sqrt{\Delta k}} \\
& \left.\left.\left(\operatorname{erf}\left[\frac{\sqrt{\alpha}(\Delta k+1)}{2 \sqrt{\Delta k}}\right]-\operatorname{erf}\left[\frac{\sqrt{\alpha} t\left(-4 \Delta k+(\Delta k+1) \frac{\tau}{t}\right)}{2 \tau \sqrt{\Delta k}}\right]\right)\right) d t\right\}
\end{aligned}
$$

proponemos un cambio de variable

$$
u=\frac{\sqrt{\alpha}}{2 \sqrt{\Delta k} \tau}(-4 \Delta k t+(\Delta k+1) \tau)
$$

donde

$$
d u=-\frac{2 \sqrt{\alpha} \sqrt{\Delta k}}{\tau} d t
$$

y los límites están dados por

$$
u(t=0)=\frac{\sqrt{\alpha}}{2 \sqrt{\Delta k} \tau}(\Delta k+1) \tau=\frac{\sqrt{\alpha}}{2 \sqrt{\Delta k}}(\Delta k+1) \quad u\left(t=\frac{\tau}{4}\right)=\frac{\sqrt{\alpha}}{2 \sqrt{\Delta k} \tau} \tau=\frac{\sqrt{\alpha}}{2 \sqrt{\Delta k}}
$$

De esta manera podemos reescribir la integral con la variable adimensional. Primero comenzamos con el protocolo de $k(t)$, buscamos reescribirla en sus variables adimensionales por lo que recordamos que $\Delta k=\frac{k_{2}-k_{1}}{k_{1}}$ de manera que podemos reescribir

$$
\left(\frac{k_{1}-k_{2}}{\tau} 4 t+k_{2}\right)=\frac{k_{1}}{\tau}(-4 \Delta k t+(1+\Delta k) \tau)
$$

Ahora lo escribimos con el cambio de variables

$$
\left(\frac{k_{1}-k_{2}}{\tau} 4 t+k_{2}\right)=\frac{k_{1}}{\tau} \frac{2 \tau \sqrt{\Delta k}}{\sqrt{\alpha}} u=\frac{2 k_{1} \sqrt{\Delta k}}{\sqrt{\alpha}} u
$$

El exponente de la primera exponencial se reescribe como un cuadrado perfecto

$$
\operatorname{Exp}\left[\frac{2 \alpha t^{2}}{\tau^{2}}\left[2 \Delta k-(\Delta k+1) \frac{\tau}{t}\right]\right]=\operatorname{Exp}\left[-\frac{\alpha(\Delta k+1)^{2}}{4 \Delta k}\right] \operatorname{Exp}\left[\frac{\alpha}{4 \Delta k \tau^{2}}(-4 \Delta k t+(\Delta k+1) \tau)^{2}\right]
$$

y de manera adimensional

$$
\operatorname{Exp}\left[\frac{2 \alpha t^{2}}{\tau^{2}}\left[2 \Delta k-(\Delta k+1) \frac{\tau}{t}\right]\right]=\operatorname{Exp}\left[-\frac{\alpha(\Delta k+1)^{2}}{4 \Delta k}\right] \operatorname{Exp}\left[u^{2}\right]
$$

En el segundo término de la integral $I_{1,1}$ la exponencial se puede reescribir como

$$
\operatorname{Exp}\left[\frac{\alpha t^{2}}{4 \Delta k \tau^{2}}\left(-4 \Delta k+(\Delta k+1) \frac{\tau}{t}\right)^{2}\right]=\operatorname{Exp}\left[u^{2}\right]
$$

Finalmente la función de error en este término se debe de reescribir tomando el cambio de variable

$$
\operatorname{erf}\left[\frac{\sqrt{\alpha} t\left(-4 \Delta k+(\Delta k+1) \frac{\tau}{t}\right.}{2 \tau \sqrt{\Delta k}}\right]=\operatorname{erf}[u]
$$

Entonces la integral $I_{1,1}$ queda de la siguiente manera

$$
I_{1,1}=-\frac{1}{2 m \gamma \epsilon\left(t_{0}\right)}\left\{\int_{\left.u_{(} t=0\right)}^{u(t=\tau / 4)}\left(\frac{2 k_{1} \sqrt{\Delta k}}{\sqrt{\alpha}} u\right)^{2}\left(e^{-\frac{\alpha(\Delta k+1)^{2}}{4 \Delta k}} e^{u^{2}}\right)\left(-\frac{\tau}{2 \sqrt{\alpha} \sqrt{\Delta k}} d u\right)\right.
$$




$$
\begin{aligned}
& \left.+\frac{\sqrt{\alpha}(1+\Delta k) \sqrt{\pi}}{2 \sqrt{\Delta k}} \int_{\left.u_{(} t=0\right)}^{u_{(t=\tau / 4)}}\left(\frac{2 k_{1} \sqrt{\Delta k}}{\sqrt{\alpha}} u\right)^{2} e^{u^{2}}\left(\operatorname{erf}\left[\frac{\sqrt{\alpha}(\Delta k+1)}{2 \sqrt{\Delta k}}\right]-\operatorname{erf}[u]\right)\left(-\frac{\tau}{2 \sqrt{\alpha} \sqrt{\Delta k}} d u\right)\right\} \\
& =-\frac{1}{2 m \gamma \epsilon\left(t_{0}\right)}\left\{-\frac{2 k_{1}^{2} \sqrt{\Delta k} \tau}{\alpha^{3 / 2}} e^{-\frac{\alpha(\Delta k+1)^{2}}{4 \Delta k}} \int_{u_{(t=0)}^{u}}^{u_{(t=\tau / 4)}} u^{2} e^{u^{2}} d u\right. \\
& \left.-\frac{k_{1}^{2}(1+\Delta k) \sqrt{\pi} \tau}{\alpha} \int_{u_{(t=0)}}^{u_{(t=\tau / 4)}} u^{2} e^{u^{2}}\left(\operatorname{erf}\left[\frac{\sqrt{\alpha}(\Delta k+1)}{2 \sqrt{\Delta k}}\right]-\operatorname{erf}[u]\right) d u\right\} \\
& =-\frac{1}{2 m \gamma \epsilon\left(t_{0}\right)}\left\{-\frac{2 k_{1}^{2} \sqrt{\Delta k} \tau}{\alpha^{3 / 2}} e^{-\frac{\alpha(\Delta k+1)^{2}}{4 \Delta k}} \int_{u_{(t=0)} u_{(t=\tau / 4)}}^{u^{2}} e^{u^{2}} d u\right. \\
& \left.-\frac{k_{1}^{2}(1+\Delta k) \tau \sqrt{\pi}}{\alpha} \operatorname{erf}\left[\frac{\sqrt{\alpha}(\Delta k+1)}{2 \sqrt{\Delta k}}\right] \int_{u_{(t=0)}}^{u_{(t=\tau / 4)}} u^{2} e^{u^{2}} d u+\frac{k_{1}^{2}(1+\Delta k) \tau \sqrt{\pi}}{\alpha} \int_{u_{(t=0)}}^{u_{(t=\tau / 4)}} u^{2} e^{u^{2}} \operatorname{erf}[u] d u\right\}
\end{aligned}
$$

Solo hay que resolver dos integrales, la solución de la primera está dada por

$$
\begin{aligned}
& \int_{u_{(t=0)}}^{u_{(t=\tau / 4)}} u^{2} e^{u^{2}} d u=\left.\left(\frac{u e^{u^{2}}}{2}-\frac{1}{4} \sqrt{\pi} e r f i[u]\right)\right|_{u_{t=0}} ^{u_{(t=\tau / 4)}} \\
= & \left(\frac{\sqrt{\alpha} e\left(\frac{\sqrt{\alpha}}{2 \sqrt{\Delta k}}\right)^{2}}{4 \sqrt{\Delta k}}-\frac{1}{4} \sqrt{\pi} \operatorname{erfi}\left[\frac{\sqrt{\alpha}}{2 \sqrt{\Delta k}}\right]\right)-\left(\frac{\sqrt{\alpha}(\Delta k+1) e^{\left(\frac{\sqrt{\alpha}}{2 \sqrt{\Delta k}}(\Delta k+1)\right)^{2}}}{4 \sqrt{\Delta k}}-\frac{1}{4} \sqrt{\pi} \operatorname{erfi}\left[\frac{\sqrt{\alpha}}{2 \sqrt{\Delta k}}(\Delta k+1)\right]\right) \\
\mathrm{y} & \int_{u_{(t=0)}}^{u_{(t=\tau / 4)}} u^{2} e^{u^{2}} \operatorname{erf}[u] d u=\left.\frac{u^{2}\left(-1+F\left[(1,1),\left(\frac{1}{2}, 2\right), u^{2}\right]\right)}{2 \sqrt{\pi}}\right|_{u_{t=0}^{u}} ^{u_{(t=\tau / 4)}} \\
= & \frac{\left(\frac{\sqrt{\alpha}}{2 \sqrt{\Delta k}}\right)^{2}\left(-1+F\left[(1,1),\left(\frac{1}{2}, 2\right),\left(\frac{\sqrt{\alpha}}{2 \sqrt{\Delta k}}\right)^{2}\right]\right)}{2 \sqrt{\pi}}-\frac{\left(\frac{\sqrt{\alpha}}{2 \sqrt{\Delta k}}(\Delta k+1)\right)^{2}\left(-1+F\left[(1,1),\left(\frac{1}{2}, 2\right),\left(\frac{\sqrt{\alpha}}{2 \sqrt{\Delta k}}(\Delta k+1)\right)^{2}\right]\right)}{2 \sqrt{\pi}}
\end{aligned}
$$

Entonces podemos escribir $I_{1,1}$

$$
\begin{aligned}
I_{1,1} & =-\frac{1}{2 m \gamma \epsilon\left(t_{0}\right)}\left\{-\frac{2 k_{1}^{2} \sqrt{\Delta k} \tau}{\alpha^{3 / 2}} e^{-\frac{\alpha(\Delta k+1)^{2}}{4 \Delta k}}\right. \\
& \left(\left(\frac{\sqrt{\alpha} e\left(\frac{\sqrt{\alpha}}{2 \sqrt{\Delta k}}\right)^{2}}{4 \sqrt{\Delta k}}-\frac{1}{4} \sqrt{\pi} e r f i\left[\frac{\sqrt{\alpha}}{2 \sqrt{\Delta k}}\right]\right)-\left(\frac{\sqrt{\alpha}(\Delta k+1) e^{\left(\frac{\sqrt{\alpha}}{2 \sqrt{\Delta k}}(\Delta k+1)\right)^{2}}}{4 \sqrt{\Delta k}}-\frac{1}{4} \sqrt{\pi} \operatorname{erfi}\left[\frac{\sqrt{\alpha}}{2 \sqrt{\Delta k}}(\Delta k+1)\right]\right)\right) \\
& -\frac{k_{1}^{2}(1+\Delta k) \tau \sqrt{\pi}}{\alpha} \operatorname{erf}\left[\frac{\sqrt{\alpha}(\Delta k+1)}{2 \sqrt{\Delta k}}\right] \\
& \left(\left(\frac{\sqrt{\alpha} e\left(\frac{\sqrt{\alpha}}{2 \sqrt{\Delta k}}\right)^{2}}{4 \sqrt{\Delta k}}-\frac{1}{4} \sqrt{\pi} e r f i\left[\frac{\sqrt{\alpha}}{2 \sqrt{\Delta k}}\right]\right)-\left(\frac{\sqrt{\alpha}(\Delta k+1) e^{\left(\frac{\sqrt{\alpha}}{2 \sqrt{\Delta k}}(\Delta k+1)\right)^{2}}}{4 \sqrt{\Delta k}}-\frac{1}{4} \sqrt{\pi} \operatorname{erfi}\left[\frac{\sqrt{\alpha}}{2 \sqrt{\Delta k}}(\Delta k+1)\right]\right)\right) \\
& +\frac{k_{1}^{2}(1+\Delta k) \tau \sqrt{\pi}}{\alpha} \\
& \left.\left(\frac{\left(\frac{\sqrt{\alpha}}{2 \sqrt{\Delta k}}\right)^{2}\left(-1+F\left[(1,1),\left(\frac{1}{2}, 2\right),\left(\frac{\sqrt{\alpha}}{2 \sqrt{\Delta k}}\right)^{2}\right]\right)}{2 \sqrt{\pi}}-\frac{\left.\left(\frac{\sqrt{\alpha}}{2 \sqrt{\Delta k}}(\Delta k+1)\right)^{2}\left(-1+F\left[(1,1),\left(\frac{1}{2}, 2\right),\left(\frac{\sqrt{\alpha}}{2 \sqrt{\Delta k}}(\Delta k+1)\right)^{2}\right]\right)\right)}{2 \sqrt{\pi}}\right)\right\} \\
& =-\frac{k_{1}^{2} \tau}{2 m \gamma \epsilon\left(t_{0}\right)}\left\{-\frac{2 \sqrt{\Delta k}}{\alpha^{3 / 2}} e^{-\frac{\alpha(\Delta k+1)^{2}}{4 \Delta k}}\right. \\
& \left(\left(\frac{\sqrt{\alpha} e\left(\frac{\sqrt{\alpha}}{2 \sqrt{\Delta k}}\right)^{2}}{4 \sqrt{\Delta k}}-\frac{1}{4} \sqrt{\pi} e r f i\left[\frac{\sqrt{\alpha}}{2 \sqrt{\Delta k}}\right]\right)-\left(\frac{\sqrt{\alpha}(\Delta k+1) e^{\left(\frac{\sqrt{\alpha}}{2 \sqrt{\Delta k}}(\Delta k+1)\right)^{2}}}{4 \sqrt{\Delta k}}-\frac{1}{4} \sqrt{\pi} \operatorname{erfi}\left[\frac{\sqrt{\alpha}}{2 \sqrt{\Delta k}}(\Delta k+1)\right]\right)\right) \\
& -\frac{(1+\Delta k) \sqrt{\pi}}{\alpha} \operatorname{erf}\left[\frac{\sqrt{\alpha}(\Delta k+1)}{2 \sqrt{\Delta k}}\right] \\
& \left(\left(\frac{\sqrt{\alpha} e\left(\frac{\sqrt{\alpha}}{2 \sqrt{\Delta k}}\right)^{2}}{4 \sqrt{\Delta k}}-\frac{1}{4} \sqrt{\pi} e r f i\left[\frac{\sqrt{\alpha}}{2 \sqrt{\Delta k}}\right]\right)-\left(\frac{\sqrt{\alpha}(\Delta k+1) e^{\left(\frac{\sqrt{\alpha}}{2 \sqrt{\Delta k}}(\Delta k+1)\right)^{2}}}{4 \sqrt{\Delta k}}-\frac{1}{4} \sqrt{\pi} \operatorname{erfi}\left[\frac{\sqrt{\alpha}}{2 \sqrt{\Delta k}}(\Delta k+1)\right]\right)\right)
\end{aligned}
$$




$$
\begin{aligned}
& +\frac{(1+\Delta k) \sqrt{\pi}}{\alpha} \\
& \left.\left(\frac{\left(\frac{\sqrt{\alpha}}{2 \sqrt{\Delta k}}\right)^{2}\left(-1+F\left[(1,1),\left(\frac{1}{2}, 2\right),\left(\frac{\sqrt{\alpha}}{2 \sqrt{\Delta k}}\right)^{2}\right]\right)}{2 \sqrt{\pi}}-\frac{\left(\frac{\sqrt{\alpha}}{2 \sqrt{\Delta k}}(\Delta k+1)\right)^{2}\left(-1+F\left[(1,1),\left(\frac{1}{2}, 2\right),\left(\frac{\sqrt{\alpha}}{2 \sqrt{\Delta k}}(\Delta k+1)\right)^{2}\right]\right)}{2 \sqrt{\pi}}\right)\right\} \\
& =-\frac{k_{1} \alpha}{2 \epsilon\left(t_{0}\right)}\left\{-\frac{2 \sqrt{\Delta k}}{\alpha^{3 / 2}} e^{-\frac{\alpha(\Delta k+1)^{2}}{4 \Delta k}}\right. \\
& \left(\left(\frac{\sqrt{\alpha} e\left(\frac{\sqrt{\alpha}}{2 \sqrt{\Delta k}}\right)^{2}}{4 \sqrt{\Delta k}}-\frac{1}{4} \sqrt{\pi} \operatorname{erfi}\left[\frac{\sqrt{\alpha}}{2 \sqrt{\Delta k}}\right]\right)-\left(\frac{\sqrt{\alpha}(\Delta k+1) e^{\left(\frac{\sqrt{\alpha}}{2 \sqrt{\Delta k}}(\Delta k+1)\right)^{2}}}{4 \sqrt{\Delta k}}-\frac{1}{4} \sqrt{\pi} \operatorname{erfi}\left[\frac{\sqrt{\alpha}}{2 \sqrt{\Delta k}}(\Delta k+1)\right]\right)\right) \\
& -\frac{(1+\Delta k) \sqrt{\pi}}{\alpha} \operatorname{erf}\left[\frac{\sqrt{\alpha}(\Delta k+1)}{2 \sqrt{\Delta k}}\right] \\
& \left(\left(\frac{\sqrt{\alpha} e\left(\frac{\sqrt{\alpha}}{2 \sqrt{\Delta k}}\right)^{2}}{4 \sqrt{\Delta k}}-\frac{1}{4} \sqrt{\pi} e r f i\left[\frac{\sqrt{\alpha}}{2 \sqrt{\Delta k}}\right]\right)-\left(\frac{\sqrt{\alpha}(\Delta k+1) e^{\left(\frac{\sqrt{\alpha}}{2 \sqrt{\Delta k}}(\Delta k+1)\right)^{2}}}{4 \sqrt{\Delta k}}-\frac{1}{4} \sqrt{\pi} \operatorname{erfi}\left[\frac{\sqrt{\alpha}}{2 \sqrt{\Delta k}}(\Delta k+1)\right]\right)\right) \\
& +\frac{(1+\Delta k) \sqrt{\pi}}{\alpha} \\
& \left.\left(\frac{\left(\frac{\sqrt{\alpha}}{2 \sqrt{\Delta k}}\right)^{2}\left(-1+F\left[(1,1),\left(\frac{1}{2}, 2\right),\left(\frac{\sqrt{\alpha}}{2 \sqrt{\Delta k}}\right)^{2}\right]\right)}{2 \sqrt{\pi}}-\frac{\left(\frac{\sqrt{\alpha}}{2 \sqrt{\Delta k}}(\Delta k+1)\right)^{2}\left(-1+F\left[(1,1),\left(\frac{1}{2}, 2\right),\left(\frac{\sqrt{\alpha}}{2 \sqrt{\Delta k}}(\Delta k+1)\right)^{2}\right]\right)}{2 \sqrt{\pi}}\right)\right\}
\end{aligned}
$$

la solución a la integral $I_{1,2}$ es directa

$$
\begin{aligned}
I_{1,2}= & \int_{0}^{\tau / 4} \frac{k_{1} q_{2}}{2 \gamma^{2} \tau}(-4 \Delta k t+(1+\Delta k) \tau) d t=\left.\frac{k_{1} q_{2}}{2 \gamma^{2} \tau}\left(\frac{-4 \Delta k t^{2}}{2}+(1+\Delta k) t \tau\right)\right|_{0} ^{\tau / 4} \\
& =\frac{k_{1} q_{2}}{2 \gamma^{2} \tau}\left(-\frac{\Delta k \tau^{2}}{8}+\frac{(1+\Delta k) \tau^{2}}{4}\right)=\frac{k_{1} q_{2} \tau(2+\Delta k)}{16 \gamma^{2}}
\end{aligned}
$$

multiplicando y dividiendo por $\frac{k_{2}}{m}$ identificamos $\epsilon\left(t_{0}\right)=\frac{\gamma k_{2}}{m q_{2}}$ y $\alpha=\frac{k_{1} \tau}{m \gamma}$ y podemos reescribirlo como

$$
=\frac{k_{1} q_{2} \tau(2+\Delta k)}{16 \gamma^{2}} \frac{k_{2} m}{k_{2} m}=\frac{\alpha k_{2}(2+\Delta k)}{16 \epsilon_{0}}=\frac{\alpha k_{1}(1+\Delta k)(2+\Delta k)}{16 \epsilon\left(t_{0}\right)}
$$

Juntando ambas integrales encontramos la expresión del calor

$$
\begin{aligned}
& \langle\Delta Q\rangle=-\frac{k_{1} \alpha}{2 \epsilon\left(t_{0}\right)}\left\{-\frac{2 \sqrt{\Delta k}}{\alpha^{3 / 2}} e^{-\frac{\alpha(\Delta k+1)^{2}}{4 \Delta k}}\right. \\
& \left(\left(\frac{\sqrt{\alpha} e\left(\frac{\sqrt{\alpha}}{2 \sqrt{\Delta k}}\right)^{2}}{4 \sqrt{\Delta k}}-\frac{1}{4} \sqrt{\pi} \operatorname{erfi}\left[\frac{\sqrt{\alpha}}{2 \sqrt{\Delta k}}\right]\right)-\left(\frac{\sqrt{\alpha}(\Delta k+1) e^{\left(\frac{\sqrt{\alpha}}{2 \sqrt{\Delta k}}(\Delta k+1)\right)^{2}}}{4 \sqrt{\Delta k}}-\frac{1}{4} \sqrt{\pi} \operatorname{erfi}\left[\frac{\sqrt{\alpha}}{2 \sqrt{\Delta k}}(\Delta k+1)\right]\right)\right) \\
& -\frac{(1+\Delta k) \sqrt{\pi}}{\alpha} \operatorname{erf}\left[\frac{\sqrt{\alpha}(\Delta k+1)}{2 \sqrt{\Delta k}}\right] \\
& \left(\left(\frac{\sqrt{\alpha} e\left(\frac{\sqrt{\alpha}}{2 \sqrt{\Delta k}}\right)^{2}}{4 \sqrt{\Delta k}}-\frac{1}{4} \sqrt{\pi} \operatorname{erfi}\left[\frac{\sqrt{\alpha}}{2 \sqrt{\Delta k}}\right]\right)-\left(\frac{\sqrt{\alpha}(\Delta k+1) e^{\left(\frac{\sqrt{\alpha}}{2 \sqrt{\Delta k}}(\Delta k+1)\right)^{2}}}{4 \sqrt{\Delta k}}-\frac{1}{4} \sqrt{\pi} \operatorname{erfi}\left[\frac{\sqrt{\alpha}}{2 \sqrt{\Delta k}}(\Delta k+1)\right]\right)\right) \\
& +\frac{(1+\Delta k) \sqrt{\pi}}{\alpha} \\
& \left.\left(\frac{\left(\frac{\sqrt{\alpha}}{2 \sqrt{\Delta k}}\right)^{2}\left(-1+F\left[(1,1),\left(\frac{1}{2}, 2\right),\left(\frac{\sqrt{\alpha}}{2 \sqrt{\Delta k}}\right)^{2}\right]\right)}{2 \sqrt{\pi}}-\frac{\left(\frac{\sqrt{\alpha}}{2 \sqrt{\Delta k}}(\Delta k+1)\right)^{2}\left(-1+F\left[(1,1),\left(\frac{1}{2}, 2\right),\left(\frac{\sqrt{\alpha}}{2 \sqrt{\Delta k}}(\Delta k+1)\right)^{2}\right]\right)}{2 \sqrt{\pi}}\right)\right\} \\
& +\frac{\alpha k_{1}(1+\Delta k)(2+\Delta k)}{16 \epsilon\left(t_{0}\right)} \\
& \text { factorizando } \frac{k_{1} \alpha}{2 \epsilon\left(t_{0}\right)}
\end{aligned}
$$


$=\frac{k_{1} \alpha}{2 \epsilon\left(t_{0}\right)}\left[+\frac{2 \sqrt{\Delta k}}{\alpha^{3 / 2}} e^{-\frac{\alpha(\Delta k+1)^{2}}{4 \Delta k}}\right.$

$\left(\left(\frac{\sqrt{\alpha} e^{\left(\frac{\sqrt{\alpha}}{2 \sqrt{\Delta k}}\right)^{2}}}{4 \sqrt{\Delta k}}-\frac{1}{4} \sqrt{\pi} e r f i\left[\frac{\sqrt{\alpha}}{2 \sqrt{\Delta k}}\right]\right)-\left(\frac{\sqrt{\alpha}(\Delta k+1) e^{\left(\frac{\sqrt{\alpha}}{2 \sqrt{\Delta k}}(\Delta k+1)\right)^{2}}}{4 \sqrt{\Delta k}}-\frac{1}{4} \sqrt{\pi} \operatorname{erfi}\left[\frac{\sqrt{\alpha}}{2 \sqrt{\Delta k}}(\Delta k+1)\right]\right)\right)$

$+\frac{(1+\Delta k) \sqrt{\pi}}{\alpha} \operatorname{erf}\left[\frac{\sqrt{\alpha}(\Delta k+1)}{2 \sqrt{\Delta k}}\right]$

$\left(\left(\frac{\sqrt{\alpha} e^{\left(\frac{\sqrt{\alpha}}{2 \sqrt{\Delta k}}\right)^{2}}}{4 \sqrt{\Delta k}}-\frac{1}{4} \sqrt{\pi} \operatorname{erfi}\left[\frac{\sqrt{\alpha}}{2 \sqrt{\Delta k}}\right]\right)-\left(\frac{\sqrt{\alpha}(\Delta k+1) e^{\left(\frac{\sqrt{\alpha}}{2 \sqrt{\Delta k}}(\Delta k+1)\right)^{2}}}{4 \sqrt{\Delta k}}-\frac{1}{4} \sqrt{\pi} \operatorname{erfi}\left[\frac{\sqrt{\alpha}}{2 \sqrt{\Delta k}}(\Delta k+1)\right]\right)\right)$

$-\frac{(1+\Delta k) \sqrt{\pi}}{\alpha}$

$\left(\frac{\left(\frac{\sqrt{\alpha}}{2 \sqrt{\Delta k}}\right)^{2}\left(-1+F\left[(1,1),\left(\frac{1}{2}, 2\right),\left(\frac{\sqrt{\alpha}}{2 \sqrt{\Delta k}}\right)^{2}\right]\right)}{2 \sqrt{\pi}}-\frac{\left(\frac{\sqrt{\alpha}}{2 \sqrt{\Delta k}}(\Delta k+1)\right)^{2}\left(-1+F\left[(1,1),\left(\frac{1}{2}, 2\right),\left(\frac{\sqrt{\alpha}}{2 \sqrt{\Delta k}}(\Delta k+1)\right)^{2}\right]\right)}{2 \sqrt{\pi}}\right)$

$\left.+\frac{(1+\Delta k)(2+\Delta k)}{8}\right]$

pero $\epsilon\left(t_{0}\right)=\frac{\gamma k_{2}}{m q_{2}}$ puede reescribirse como $\epsilon\left(t_{0}\right)=\frac{\gamma k_{1}(\Delta k+1)}{m q_{1}(\Delta q+1)}=\frac{\varepsilon(\Delta k+1)}{(\Delta q+1)}$ de esta manera la expresión final del calor es la siguiente

$$
\begin{aligned}
\langle\Delta Q\rangle & =\frac{k_{1} \alpha(1+\Delta q)}{2 \varepsilon(1+\Delta k)}\left[+\frac{2 \sqrt{\Delta k}}{\alpha^{3 / 2}} e^{-\frac{\alpha(\Delta k+1)^{2}}{4 \Delta k}}\right. \\
& \left(\left(\frac{\sqrt{\alpha} e\left(\frac{\sqrt{\alpha}}{2 \sqrt{\Delta k}}\right)^{2}}{4 \sqrt{\Delta k}}-\frac{1}{4} \sqrt{\pi} \operatorname{erfi}\left[\frac{\sqrt{\alpha}}{2 \sqrt{\Delta k}}\right]\right)-\left(\frac{\sqrt{\alpha}(\Delta k+1) e^{\left(\frac{\sqrt{\alpha}}{2 \sqrt{\Delta k}}(\Delta k+1)\right)^{2}}}{4 \sqrt{\Delta k}}-\frac{1}{4} \sqrt{\pi} \operatorname{erfi}\left[\frac{\sqrt{\alpha}}{2 \sqrt{\Delta k}}(\Delta k+1)\right]\right)\right) \\
& +\frac{(1+\Delta k) \sqrt{\pi}}{\alpha} \operatorname{erf}\left[\frac{\sqrt{\alpha}(\Delta k+1)}{2 \sqrt{\Delta k}}\right] \\
& \left(\left(\frac{\sqrt{\alpha} e\left(\frac{\sqrt{\alpha}}{2 \sqrt{\Delta k}}\right)^{2}}{4 \sqrt{\Delta k}}-\frac{1}{4} \sqrt{\pi} \operatorname{erfi}\left[\frac{\sqrt{\alpha}}{2 \sqrt{\Delta k}}\right]\right)-\left(\frac{\sqrt{\alpha}(\Delta k+1) e^{\left(\frac{\sqrt{\alpha}}{2 \sqrt{\Delta k}}(\Delta k+1)\right)^{2}}}{4 \sqrt{\Delta k}}-\frac{1}{4} \sqrt{\pi} \operatorname{erfi}\left[\frac{\sqrt{\alpha}}{2 \sqrt{\Delta k}}(\Delta k+1)\right]\right)\right) \\
& -\frac{(1+\Delta k) \sqrt{\pi}}{\alpha} \\
& \left(\frac{\left(\frac{\sqrt{\alpha}}{2 \sqrt{\Delta k}}\right)^{2}\left(-1+F\left[(1,1),\left(\frac{1}{2}, 2\right),\left(\frac{\sqrt{\alpha}}{2 \sqrt{\Delta k}}\right)^{2}\right]\right)}{2 \sqrt{\pi}}-\frac{\left(\frac{\sqrt{\alpha}}{2 \sqrt{\Delta k}}(\Delta k+1)\right)^{2}\left(-1+F\left[(1,1),\left(\frac{1}{2}, 2\right),\left(\frac{\sqrt{\alpha}}{2 \sqrt{\Delta k}}(\Delta k+1)\right)^{2}\right]\right)}{2 \sqrt{\pi}}\right) \\
& \left.+\frac{(1+\Delta k)(2+\Delta k)}{8}\right]
\end{aligned}
$$

Podemos escribir una ecuació adimensional para el calor ya que $\frac{k_{1}}{\varepsilon}$ tiene unidades de Joule las mismas que el calor, escribiendo $\frac{\varepsilon\langle\Delta Q\rangle}{k_{1}}=\langle\Delta Q\rangle^{*}$ llegamos a una expresión adimensional para el calor

$$
\begin{aligned}
\left\langle\Delta Q_{1 \rightarrow 2}\right\rangle^{*} & =\frac{\alpha(1+\Delta q)}{2(1+\Delta k)}\left[+\frac{2 \sqrt{\Delta k}}{\alpha^{3 / 2}} e^{-\frac{\alpha(\Delta k+1)^{2}}{4 \Delta k}}\right. \\
& \left(\left(\frac{\sqrt{\alpha} e^{\left(\frac{\sqrt{\alpha}}{2 \sqrt{\Delta k}}\right)^{2}}}{4 \sqrt{\Delta k}}-\frac{1}{4} \sqrt{\pi} \operatorname{erfi}\left[\frac{\sqrt{\alpha}}{2 \sqrt{\Delta k}}\right]\right)-\left(\frac{\sqrt{\alpha}(\Delta k+1) e^{\left(\frac{\sqrt{\alpha}}{2 \sqrt{\Delta k}}(\Delta k+1)\right)^{2}}}{4 \sqrt{\Delta k}}-\frac{1}{4} \sqrt{\pi} \operatorname{erfi}\left[\frac{\sqrt{\alpha}}{2 \sqrt{\Delta k}}(\Delta k+1)\right]\right)\right) \\
& +\frac{(1+\Delta k) \sqrt{\pi}}{\alpha} \operatorname{erf}\left[\frac{\sqrt{\alpha}(\Delta k+1)}{2 \sqrt{\Delta k}}\right] \\
& \left(\left(\frac{\sqrt{\alpha} e^{\left(\frac{\sqrt{\alpha}}{2 \sqrt{\Delta k}}\right)^{2}}}{4 \sqrt{\Delta k}}-\frac{1}{4} \sqrt{\pi} \operatorname{erfi}\left[\frac{\sqrt{\alpha}}{2 \sqrt{\Delta k}}\right]\right)-\left(\frac{\sqrt{\alpha}(\Delta k+1) e^{\left(\frac{\sqrt{\alpha}}{2 \sqrt{\Delta k}}(\Delta k+1)\right)^{2}}}{4 \sqrt{\Delta k}}-\frac{1}{4} \sqrt{\pi} \operatorname{erfi}\left[\frac{\sqrt{\alpha}}{2 \sqrt{\Delta k}}(\Delta k+1)\right]\right)\right) \\
& -\frac{(1+\Delta k) \sqrt{\pi}}{\alpha}
\end{aligned}
$$




$$
\begin{aligned}
& \left(\frac{\left(\frac{\sqrt{\alpha}}{2 \sqrt{\Delta k}}\right)^{2}\left(-1+F\left[(1,1),\left(\frac{1}{2}, 2\right),\left(\frac{\sqrt{\alpha}}{2 \sqrt{\Delta k}}\right)^{2}\right]\right)}{2 \sqrt{\pi}}-\frac{\left(\frac{\sqrt{\alpha}}{2 \sqrt{\Delta k}}(\Delta k+1)\right)^{2}\left(-1+F\left[(1,1),\left(\frac{1}{2}, 2\right),\left(\frac{\sqrt{\alpha}}{2 \sqrt{\Delta k}}(\Delta k+1)\right)^{2}\right]\right)}{2 \sqrt{\pi}}\right) \\
& \left.+\frac{(1+\Delta k)(2+\Delta k)}{8}\right]
\end{aligned}
$$

Para analizar el resultado damos los valores numéricos a las variables adimensionales.

$$
\Delta k=1 \quad \Delta q=1
$$

El límite cuando $\alpha$ tiende a infinito

$$
\lim _{\alpha \rightarrow \infty}\left\langle\Delta Q_{1 \rightarrow 2}\right\rangle^{*}=\frac{\log (2)}{2}
$$

Al graficar el promedio del calor encontramos el siguiente comportamiento

\section{D.2. 2 a 3}

Este proceso es un cambio en la intensidad del ruido manteniendo la rigidez del potencial constante

$$
\begin{aligned}
& k(t)=k_{1} \\
& q(t)=\frac{q_{1}-q_{2}}{\tau} 4\left(t-\frac{\tau}{4}\right)+q_{2}
\end{aligned}
$$

y la solución de $\epsilon$ está dada por

$$
\frac{1}{\epsilon_{2 \rightarrow 3}(t)}=\frac{1}{\epsilon\left(t_{0}\right)}\left(e^{-\frac{2 \alpha t\left(1-\frac{\tau}{4 t}\right)}{\tau}}-\frac{4 t \alpha \Delta q}{\alpha \tau(1+\Delta q)}-\frac{2 \Delta q\left[-1+e^{-\frac{2 \alpha t}{\tau}\left(1-\frac{\tau}{4 t}\right)}\right]+\alpha\left[-1-2 \Delta q+e^{-\frac{2 \alpha t}{\tau}\left(1-\frac{\tau}{4 t}\right)}(1+\Delta q)\right]}{\alpha(1+\Delta q)}\right)
$$

por lo tanto para encontrar el calor es necesario resolver la siguiente integral

$$
\left\langle\Delta Q_{2 \rightarrow 3}\right\rangle=\int_{\tau / 4}^{2 \tau / 4}\left[-\frac{k_{1}^{2}}{2 m \gamma \epsilon_{2 \rightarrow 3}(t)}+\frac{k_{1}\left(\frac{q_{1}-q_{2}}{\tau} 4\left(t-\frac{\tau}{4}\right)+q_{2}\right)}{2 \gamma^{2}}\right] d t
$$

lo podemos reescribir como dos integrales

$$
\left\langle\Delta Q_{2 \rightarrow 3}\right\rangle=\underbrace{\int_{\tau / 4}^{2 \tau / 4}-\frac{k_{1}^{2}}{2 m \gamma \epsilon_{2 \rightarrow 3}(t)} d t}_{I_{2,1}}+\underbrace{\int_{\tau / 4}^{2 \tau / 4} \frac{k_{1}\left(\frac{q_{1}-q_{2}}{\tau} 4\left(t-\frac{\tau}{4}\right)+q_{2}\right)}{2 \gamma^{2}} d t}_{I_{2,2}}
$$

Resolveremos la primera integral

$$
\begin{aligned}
& I_{2,1}=-\frac{k_{1}^{2}}{2 m \gamma} \int_{\tau / 4}^{2 \tau / 4} \frac{1}{\epsilon_{2 \rightarrow 3}(t)} d t \\
& =-\frac{k_{1}^{2}}{2 m \gamma \epsilon\left(t_{0}\right)} \int_{\tau / 4}^{2 \tau / 4}\left(e^{-\frac{2 \alpha t\left(1-\frac{\tau}{4 t}\right)}{\tau}}-\frac{4 t \alpha \Delta q}{\alpha \tau(1+\Delta q)}\right. \\
& \left.-\frac{2 \Delta q\left[-1+e^{-\frac{2 \alpha t}{\tau}\left(1-\frac{\tau}{4 t}\right)}\right]+\alpha\left[-1-2 \Delta q+e^{-\frac{2 \alpha t}{\tau}\left(1-\frac{\tau}{4 t}\right)}(1+\Delta q)\right]}{\alpha(1+\Delta q)}\right) d t \\
& =-\frac{k_{1}^{2}}{2 m \gamma \epsilon\left(t_{0}\right)}\left\{\int_{\tau / 4}^{2 \tau / 4}\left(e^{-\frac{2 \alpha t\left(1-\frac{\tau}{4 t}\right)}{\tau}}\right) d t-\int_{\tau / 4}^{2 \tau / 4}\left(\frac{4 t \alpha \Delta q}{\alpha \tau(1+\Delta q)}\right) d t\right. \\
& \left.-\int_{\tau / 4}^{2 \tau / 4}\left(\frac{2 \Delta q\left[-1+e^{-\frac{2 \alpha t}{\tau}\left(1-\frac{\tau}{4 t}\right)}\right]+\alpha\left[-1-2 \Delta q+e^{-\frac{2 \alpha t}{\tau}\left(1-\frac{\tau}{4 t}\right)}(1+\Delta q)\right]}{\alpha(1+\Delta q)}\right) d t\right\}
\end{aligned}
$$

El primer término es simple de integrar, la solución es la siguiente

$$
\int_{\tau / 4}^{2 \tau / 4}\left(e^{-\frac{2 \alpha t\left(1-\frac{\tau}{4 t}\right)}{\tau}}\right) d t=\frac{\tau}{2 \alpha}\left(1-e^{-\frac{\alpha}{2}}\right)
$$


La solución del segundo término es

$$
\int_{\tau / 4}^{2 \tau / 4}\left(\frac{4 t \alpha \Delta q}{\alpha \tau(1+\Delta q)}\right) d t=\frac{3 \Delta q \tau}{8(1+\Delta q)}
$$

El tercer término está dado por

$$
\begin{aligned}
& \int_{\tau / 4}^{2 \tau / 4}\left(\frac{2 \Delta q\left[-1+e^{-\frac{2 \alpha t}{\tau}\left(1-\frac{\tau}{4 t}\right)}\right]+\alpha\left[-1-2 \Delta q+e^{-\frac{2 \alpha t}{\tau}\left(1-\frac{\tau}{4 t}\right)}(1+\Delta q)\right]}{\alpha(1+\Delta q)}\right) d t \\
& =\frac{-4 \tau e^{-\frac{\alpha}{2}}(\alpha+(2+\alpha) \Delta q)-\tau(-8 \Delta q+\alpha(-4+\alpha(2+\Delta q)))}{8 \alpha^{2}(1+\Delta q)}
\end{aligned}
$$

al juntar todos los términos encontramos la solución a la integral $I_{2,1}$

$$
\begin{aligned}
I_{2,1} & =-\frac{k_{1}^{2}}{2 m \gamma \epsilon\left(t_{0}\right)}\left(\frac{\tau}{2 \alpha}\left(1-e^{-\frac{\alpha}{2}}\right)-\frac{3 \Delta q \tau}{8(1+\Delta q)}\right. \\
& \left.-\frac{-4 \tau e^{-\frac{\alpha}{2}}(\alpha+(2+\alpha) \Delta q)-\tau(-8 \Delta q+\alpha(-4+\alpha(2+\Delta q)))}{8 \alpha^{2}(1+\Delta q)}\right) \\
& \text { factorizando } \frac{\tau}{8 \alpha^{2}(1+\Delta q)} \\
& =-\frac{k_{1}^{2}}{2 m \gamma \epsilon\left(t_{0}\right)} \frac{\tau}{8 \alpha^{2}(1+\Delta q)}\left(4 \alpha(1+\Delta q)\left(1-e^{-\frac{\alpha}{2}}\right)-3 \alpha^{2} \Delta q\right. \\
& \left.+4 e^{-\frac{\alpha}{2}}(\alpha+(2+\alpha) \Delta q)+(-8 \Delta q+\alpha(-4+\alpha(2+\Delta q)))\right) \\
& =-\frac{k_{1}^{2} \tau}{16 \alpha^{2} m \gamma \epsilon\left(t_{0}\right)(1+\Delta q)}\left(8 \Delta q\left(e^{-\frac{\alpha}{2}}-1\right)+4 \alpha \Delta q+\alpha^{2}(2+\Delta q)\right)
\end{aligned}
$$

identificamos $\alpha$

$$
=-\frac{k_{1} \alpha}{16 \alpha^{2} \epsilon\left(t_{0}\right)(1+\Delta q)}\left(8 \Delta q\left(e^{-\frac{\alpha}{2}}-1\right)+4 \alpha \Delta q+\alpha^{2}(2+\Delta q)\right)
$$

La solución a la integral $I_{2,2}$ de igual manera es sencilla.

$$
\int_{\tau / 4}^{2 \tau / 4} \frac{k_{1}\left(\frac{q_{1}-q_{2}}{\tau} 4\left(t-\frac{\tau}{4}\right)+q_{2}\right)}{2 \gamma^{2}} d t
$$

en términos de la variable adimensional $\Delta q=\frac{q_{2}-q_{1}}{q_{1}}$

$$
\begin{aligned}
& =\left.\frac{k_{1} q_{1}}{2 \gamma^{2}}\left[2 \frac{-\Delta q}{\tau} t^{2}+(2 \Delta q+1) t\right]\right|_{\tau / 4} ^{2 \tau / 4} \\
& =\frac{k_{1} q_{1}}{2 \gamma^{2}}\left[2 \frac{-\Delta q}{\tau}\left(\frac{2 \tau}{4}\right)^{2}+(2 \Delta q+1) \frac{2 \tau}{4}-2 \frac{-\Delta q}{\tau}\left(\frac{\tau}{4}\right)^{2}-(2 \Delta q+1) \frac{\tau}{4}\right] \\
& =\frac{k_{1} q_{1} \tau}{2 \gamma^{2}}\left[-\frac{4 \Delta q}{8}+\Delta q+\frac{2}{4}+\frac{\Delta q}{8}-\frac{4 \Delta q}{8}-\frac{1}{4}\right]=\frac{k_{1} q_{1} \tau m}{16 m \gamma^{2}}[\Delta q+2]
\end{aligned}
$$

identificamos $\alpha$ y recordamos que la condición inicial de este proceso es $\epsilon_{0}=\frac{\gamma k_{1}}{m q_{2}}$ multiplicando y dividiendo por $q_{2}$ y $k_{1}$

$$
=\frac{k_{1} q_{2} \alpha q_{1} m}{16 k_{1} q_{2} \gamma}[\Delta q+2]=\frac{k_{1} \alpha}{16 \epsilon_{0}(1+\Delta q)}[\Delta q+2]
$$

al sumar $I_{2,1}$ y $I_{2,2}$ encontramos la expresión del calor

$$
\begin{aligned}
\left\langle\Delta Q_{2 \rightarrow 3}\right\rangle & =-\frac{k_{1} \alpha}{16 \alpha^{2} \epsilon\left(t_{0}\right)(1+\Delta q)}\left(8 \Delta q\left(e^{-\frac{\alpha}{2}}-1\right)+4 \alpha \Delta q+\alpha^{2}(2+\Delta q)\right)+\frac{k_{1} \alpha}{16 \epsilon_{0}(1+\Delta q)}[\Delta q+2] \\
& =\frac{k_{1}}{16 \epsilon\left(t_{0}\right) \alpha(1+\Delta q)}\left[-8 \Delta q\left(e^{-\frac{\alpha}{2}}-1\right)-4 \alpha \Delta q-\alpha^{2}(2+\Delta q)+\alpha^{2}(\Delta q+2)\right] \\
& =\frac{k_{1}}{16 \epsilon\left(t_{0}\right) \alpha(1+\Delta q)}\left[-8 \Delta q\left(e^{-\frac{\alpha}{2}}-1\right)-4 \alpha \Delta q\right]
\end{aligned}
$$


Pero notamos que $\epsilon\left(t_{0}\right)=\frac{\gamma k_{1}}{m q_{2}}=\frac{\gamma k_{1}}{m q 1(1+\Delta q)}=\frac{\varepsilon}{(1+\Delta q)}$

$$
\left\langle\Delta Q_{2 \rightarrow 3}\right\rangle=\frac{k_{1}}{16 \frac{\varepsilon}{(1+\Delta q)} \alpha(1+\Delta q)}\left[-8 \Delta q\left(e^{-\frac{\alpha}{2}}-1\right)-4 \alpha \Delta q\right]=\frac{k_{1}}{16 \varepsilon \alpha}\left[-8 \Delta q\left(e^{-\frac{\alpha}{2}}-1\right)-4 \alpha \Delta q\right]
$$

Como hemos visto antes $\frac{k_{1}}{\varepsilon}$ tiene unidades de energía por lo que podemos escribir un calor adimensional $\frac{\varepsilon\left\langle\Delta Q_{2 \rightarrow 3}\right\rangle}{k_{1}}=$ $\left\langle\Delta Q_{2 \rightarrow 3}\right\rangle^{*}$

$$
\left\langle\Delta Q_{2 \rightarrow 3}\right\rangle^{*}=\frac{1}{16 \alpha}\left[-8 \Delta q\left(e^{-\frac{\alpha}{2}}-1\right)-4 \alpha \Delta q\right]
$$

La expresión es muy sencilla esto nos permite encontrar en límite del calor cuando se toma $\alpha$ muy grande sin la necesidad de dar valores numéricos.

$$
\lim _{\alpha \rightarrow \infty}\left\langle\Delta Q_{2 \rightarrow 3}\right\rangle^{*}=-\frac{\Delta q}{4}
$$

Dando valor numérico a las variables adimensionales $\Delta k=1, \Delta Q=1$ podemos graficar

\section{D.3. 3 a 4}

Este proceso es un cambio en la rigidez del potencial, mientras se mantiene un ruido constante, el protocolo es

$$
\begin{aligned}
& k(t)=\frac{k_{2}-k_{1}}{\tau} 4\left(t-\frac{2 \tau}{4}\right)+k_{1}=\frac{k_{1} \Delta k}{\tau} 4\left(t-\frac{2 \tau}{4}\right)+k_{1}=k_{1}\left(\frac{\Delta k}{\tau} 4\left(t-\frac{2 \tau}{4}\right)+1\right) \\
& q(t)=q_{1}
\end{aligned}
$$

y la forma de $\epsilon(t)$ durante este proceso es el siguiente

$$
\frac{1}{\epsilon_{3 \rightarrow 4}(t)}=\frac{1}{\epsilon\left(t_{0}\right)}\left(e^{-\frac{\alpha t^{2}\left(2-\frac{\tau}{t}\right)\left(2 \Delta k+\frac{\tau}{t}-\Delta k \frac{\tau}{t}\right)}{\tau^{2}}}-\frac{\sqrt{\alpha} \sqrt{\pi} e^{-\frac{\alpha t^{2}}{4 \Delta k \tau^{2}}\left(4 \Delta k-2 \Delta k \frac{\tau}{t}+\frac{\tau}{t}\right)^{2}}}{2 \sqrt{\Delta k}}\left[\operatorname{erfi}\left(\frac{\sqrt{\alpha}}{2 \sqrt{\Delta k}}\right)-\operatorname{erfi}\left(\frac{\sqrt{\alpha} t\left(4 \Delta k+\frac{\tau}{t}-2 \Delta k \frac{\tau}{t}\right)}{2 \tau \sqrt{\Delta k}}\right)\right]\right)
$$

Para encontrar el calor en necesario resolver la siguiente integral

$$
\begin{aligned}
\left\langle\Delta Q_{3 \rightarrow 4}\right\rangle & =\int_{\frac{2 \tau}{4}}^{\frac{3 \tau}{4}}\left[-\frac{k_{1}^{2}\left(\frac{\Delta k}{\tau} 4\left(t-\frac{2 \tau}{4}\right)+1\right)^{2}}{2 m \gamma \epsilon_{3 \rightarrow 4}(t)}+\frac{q_{1} k_{1}\left(\frac{\Delta k}{\tau} 4\left(t-\frac{2 \tau}{4}\right)+1\right)}{2 \gamma^{2}}\right] d t \\
& =\underbrace{\int_{\frac{2 \tau}{4}}^{\frac{3 \tau}{4}}\left[-\frac{k_{1}^{2}\left(\frac{\Delta k}{\tau} 4\left(t-\frac{2 \tau}{4}\right)+1\right)^{2}}{2 m \gamma \epsilon_{3 \rightarrow 4}(t)}\right] d t}_{I_{3,1}}+\underbrace{\int_{\frac{2 \tau}{4}}^{\frac{3 \tau}{4}}\left[\frac{q_{1} k_{1}\left(\frac{\Delta k}{\tau} 4\left(t-\frac{2 \tau}{4}\right)+1\right)}{2 \gamma^{2}}\right]}_{I_{3,2}} d t
\end{aligned}
$$

Primero trabajaremos con $I_{3,1}$

$$
\begin{aligned}
I_{3,1} & =-\frac{k_{1}^{2}}{2 m \gamma} \int_{\frac{2 \tau}{4}}^{\frac{3 \tau}{4}}\left[\frac{\left(\frac{\Delta k}{\tau} 4\left(t-\frac{2 \tau}{4}\right)+1\right)^{2}}{\epsilon_{3 \rightarrow 4}(t)}\right] d t \\
& =-\frac{k_{1}^{2}}{2 m \gamma} \int_{\frac{2 \tau}{4}}^{\frac{3 \tau}{4}}\left(\frac{\Delta k}{\tau} 4\left(t-\frac{2 \tau}{4}\right)+1\right)^{2}\left(\frac { 1 } { \epsilon ( t _ { 0 } ) } \left(e^{-\frac{\alpha t^{2}}{\tau^{2}}\left(2-\frac{\tau}{t}\right)\left(2 \Delta k+\frac{\tau}{t}-\Delta k \frac{\tau}{t}\right)}\right.\right. \\
& \left.\left.-\frac{\sqrt{\alpha} \sqrt{\pi} e^{-\frac{\alpha t^{2}}{4 \Delta k \tau^{2}}\left(4 \Delta k-2 \Delta k \frac{\tau}{t}+\frac{\tau}{t}\right)^{2}}}{2 \sqrt{\Delta k}}\left[\operatorname{erfi}\left(\frac{\sqrt{\alpha}}{2 \sqrt{\Delta k}}\right)-\operatorname{erfi}\left(\frac{\sqrt{\alpha} t\left(4 \Delta k+\frac{\tau}{t}-2 \Delta k \frac{\tau}{t}\right)}{2 \tau \sqrt{\Delta k}}\right)\right]\right)\right) d t
\end{aligned}
$$

el exponente de la primera exponencial la podemos reescribir como un binomio cuadrado perfecto de la siguiente manera

$$
e^{-\frac{\alpha t^{2}}{\tau^{2}}\left(2-\frac{\tau}{t}\right)\left(2 \Delta k+\frac{\tau}{t}-\Delta k \frac{\tau}{t}\right)}=e^{\frac{\alpha}{4 \Delta k}} e^{-\frac{\alpha}{4 \Delta k \tau^{2}}(4 t \Delta k+(1-2 \Delta k) \tau)^{2}}
$$

proponemos un cambio de variable

$$
u=\frac{\sqrt{\alpha}}{2 \sqrt{\Delta k} \tau}(4 \Delta k t+(1-2 \Delta k) \tau)
$$


donde

$$
d u=\frac{2 \sqrt{\alpha} \sqrt{\Delta k}}{\tau} d t
$$

y los límites están dados por

$$
\begin{aligned}
& u\left(t=\frac{2 \tau}{4}\right)=\frac{\sqrt{\alpha}}{2 \sqrt{\Delta k} \tau}(2 \Delta k \tau+(1-2 \Delta k) \tau)=\frac{\sqrt{\alpha}}{2 \sqrt{\Delta k}} \\
& u\left(t=\frac{3 \tau}{4}\right)=\frac{\sqrt{\alpha}}{2 \sqrt{\Delta k} \tau}(3 \Delta k \tau+(1-2 \Delta k) \tau)=\frac{\sqrt{\alpha}}{2 \sqrt{\Delta k} \tau}(\Delta k+1) \tau
\end{aligned}
$$

Reescribimos cada uno de los términos en función de la variable adimenisonal

$$
\begin{aligned}
& \left(\frac{\Delta k}{\tau} 4\left(t-\frac{2 \tau}{4}\right)+1\right)^{2}=\frac{1}{\tau^{2}}(4 \Delta k t-2 \Delta k \tau+\tau)^{2}=\frac{4 \Delta k}{\alpha} u^{2} \\
& \operatorname{Exp}\left[\frac{\alpha}{4 \Delta k}\right] \operatorname{Exp}\left[-\frac{\alpha}{4 \Delta k \tau^{2}}(4 t \Delta k+(1-2 \Delta k) \tau)^{2}\right]=e^{\frac{\alpha}{4 \Delta k}} e^{-u^{2}} \\
& \operatorname{Exp}\left[-\frac{\alpha t^{2}}{4 \Delta k \tau^{2}}\left(4 \Delta k-2 \Delta k \frac{\tau}{t}+\frac{\tau}{t}\right)^{2}\right]=e^{-u^{2}} \\
& \text { y finalmente } \\
& \operatorname{erfi}\left(\frac{\sqrt{\alpha} t\left(4 \Delta k+\frac{\tau}{t}-2 \Delta k \frac{\tau}{t}\right)}{2 \tau \sqrt{\Delta k}}\right)=\operatorname{erfi}(u)
\end{aligned}
$$

entonces la integral $I_{3,1}$ la podemos escribir de la siguiente manera

$$
\begin{aligned}
I_{3,1} & =-\frac{k_{1}^{2}}{2 m \gamma \epsilon\left(t_{0}\right)} \int_{u\left(t=\frac{2 \tau}{4}\right)}^{u\left(t=\frac{3 \tau}{4}\right)} \frac{4 \Delta k}{\alpha} u^{2}\left(e^{\frac{\alpha}{4 \Delta k}} e^{-u^{2}}-\frac{\sqrt{\alpha} \sqrt{\pi} e^{-u^{2}}}{2 \sqrt{\Delta k}}\left[\operatorname{erfi}\left(\frac{\sqrt{\alpha}}{2 \sqrt{\Delta k}}\right)-\operatorname{erfi}(u)\right]\right) \frac{\tau}{2 \sqrt{\alpha} \sqrt{\Delta k}} d u \\
& =-\frac{4 k_{1}^{2} \Delta k \tau}{2 m \gamma \epsilon\left(t_{0}\right) \alpha(2 \sqrt{\alpha} \sqrt{\Delta k})} \int_{u\left(t=\frac{2 \tau}{4}\right)}^{u\left(t=\frac{3 \tau}{4}\right)} u^{2}\left(e^{\frac{\alpha}{4 \Delta k}} e^{-u^{2}}-\frac{\sqrt{\alpha} \sqrt{\pi} e^{-u^{2}}}{2 \sqrt{\Delta k}}\left[\operatorname{erfi}\left(\frac{\sqrt{\alpha}}{2 \sqrt{\Delta k}}\right)-\operatorname{erfi}(u)\right]\right) d u \\
& =-\frac{k_{1}^{2} \tau \sqrt{\Delta k}}{m \gamma \epsilon\left(t_{0}\right) \alpha^{3 / 2}} \int_{u\left(t=\frac{2 \tau}{4}\right)}^{u\left(t=\frac{3 \tau}{4}\right)}\left(u^{2} e^{\frac{\alpha}{4 \Delta k}} e^{-u^{2}}-\frac{\sqrt{\alpha} \sqrt{\pi} u^{2} e^{-u^{2}}}{2 \sqrt{\Delta k}}\left[\operatorname{erfi}\left(\frac{\sqrt{\alpha}}{2 \sqrt{\Delta k}}\right)-\operatorname{erfi}(u)\right]\right) d u \\
& =-\frac{k_{1}^{2} \tau \sqrt{\Delta k}}{m \gamma \epsilon\left(t_{0}\right) \alpha^{3 / 2}}\left\{e^{\frac{\alpha}{4 \Delta k}} \int_{u\left(t=\frac{2 \tau}{4}\right)}^{u\left(t=\frac{3 \tau}{4}\right)} u^{2} e^{-u^{2}} d u-\frac{\sqrt{\alpha} \sqrt{\pi}}{2 \sqrt{\Delta k}} \operatorname{erfi}\left(\frac{\sqrt{\alpha}}{2 \sqrt{\Delta k}}\right) \int_{u\left(t=\frac{2 \tau}{4}\right)}^{u\left(t=\frac{3 \tau}{4}\right)} u^{2} e^{-u^{2}} d u\right. \\
& \left.+\frac{\sqrt{\alpha} \sqrt{\pi}}{2 \sqrt{\Delta k}} \int_{u\left(t=\frac{2 \tau}{4}\right)}^{u\left(t=\frac{3 \tau}{4}\right)} u^{2} e^{-u^{2}} \operatorname{erfi}(u) d u\right\}
\end{aligned}
$$

La solución se resume a resolver dos integrales, primero

$$
\begin{aligned}
& \int_{u\left(t=\frac{2 \tau}{4}\right)}^{u\left(t=\frac{3 \tau}{4}\right)} u^{2} e^{-u^{2}} d u=\left.e^{\frac{\alpha}{4 \Delta k}}\left[-\frac{u e^{-u^{2}}}{2}+\frac{\sqrt{\pi}}{4} \operatorname{erf}(u)\right]\right|_{u\left(t=\frac{2 \tau}{4}\right)} ^{u\left(t=\frac{3 \tau}{4}\right)} \\
& =-\frac{\frac{\sqrt{\alpha}}{2 \sqrt{\Delta k} \tau}(\Delta k+1) \tau e^{-\left(\frac{\sqrt{\alpha}}{2 \sqrt{\Delta k} \tau}(\Delta k+1) \tau\right)^{2}}}{2}+\frac{\sqrt{\pi}}{4} \operatorname{erf}\left(\frac{\sqrt{\alpha}}{2 \sqrt{\Delta k} \tau}(\Delta k+1) \tau\right) \\
& +\frac{\frac{\sqrt{\alpha}}{2 \sqrt{\Delta k} \tau} \tau e^{-\left(\frac{\sqrt{\alpha}}{2 \sqrt{\Delta k} \tau} \tau\right)^{2}}}{2}-\frac{\sqrt{\pi}}{4} \operatorname{erf}\left(\frac{\sqrt{\alpha}}{2 \sqrt{\Delta k} \tau} \tau\right) \\
& =\frac{\sqrt{\alpha}(\Delta k+1) e^{-\frac{\alpha}{4 \Delta k}(\Delta k+1)^{2}}}{4 \sqrt{\Delta k}}+\frac{\sqrt{\pi}}{4} \operatorname{erf}\left(\frac{\sqrt{\alpha}}{2 \sqrt{\Delta k}}(\Delta k+1)\right)+\frac{\sqrt{\alpha} e^{-\frac{\alpha}{4 \Delta k}}}{4 \sqrt{\Delta k}}-\frac{\sqrt{\pi}}{4} \operatorname{erf}\left(\frac{\sqrt{\alpha}}{2 \sqrt{\Delta k}}\right) \\
& =\frac{\sqrt{\alpha}}{4 \sqrt{\Delta k}}\left\{(\Delta k+1) e^{-\frac{\alpha}{4 \Delta k}(\Delta k+1)^{2}}+\frac{\sqrt{\Delta k} \sqrt{\pi}}{\sqrt{\alpha}} \operatorname{erf}\left(\frac{\sqrt{\alpha}}{2 \sqrt{\Delta k}}(\Delta k+1)\right)+e^{-\frac{\alpha}{4 \Delta k}}-\frac{\sqrt{\Delta k} \sqrt{\pi}}{\sqrt{\alpha}} \operatorname{erf}\left(\frac{\sqrt{\alpha}}{2 \sqrt{\Delta k}}\right)\right\}
\end{aligned}
$$

La segunda integral a resolver es

$$
\int_{u\left(t=\frac{2 \tau}{4}\right)}^{u\left(t=\frac{3 \tau}{4}\right)} u^{2} e^{-u^{2}} \operatorname{erfi}(u) d u=\left.\frac{u^{2}\left(1-{ }_{2} F_{2}\left[1,1 ; \frac{1}{2}, 2 ;-u^{2}\right]\right)}{2 \sqrt{\pi}}\right|_{u\left(t=\frac{2 \tau}{4}\right)} ^{u\left(t=\frac{3 \tau}{4}\right)}
$$




$$
\begin{aligned}
& =\frac{\left(\frac{\sqrt{\alpha}}{2 \sqrt{\Delta k} \tau}(\Delta k+1) \tau\right)^{2}\left(1-{ }_{2} F_{2}\left[1,1 ; \frac{1}{2}, 2 ;-\left(\frac{\sqrt{\alpha}}{2 \sqrt{\Delta k} \tau}(\Delta k+1) \tau\right)^{2}\right]\right)}{2 \sqrt{\pi}} \\
& -\frac{\left(\frac{\sqrt{\alpha}}{2 \sqrt{\Delta k}}\right)^{2}\left(1-{ }_{2} F_{2}\left[1,1 ; \frac{1}{2}, 2 ;-\left(\frac{\sqrt{\alpha}}{2 \sqrt{\Delta k}}\right)^{2}\right]\right)}{2 \sqrt{\pi}} \\
& =\frac{\frac{\alpha}{4 \Delta k}(\Delta k+1)^{2}\left(1-{ }_{2} F_{2}\left[1,1 ; \frac{1}{2}, 2 ;-\frac{\alpha}{4 \Delta k}(\Delta k+1)^{2}\right]\right)}{2 \sqrt{\pi}}-\frac{\frac{\alpha}{4 \Delta k}\left(1-{ }_{2} F_{2}\left[1,1 ; \frac{1}{2}, 2 ;-\frac{\alpha}{4 \Delta k}\right]\right)}{2 \sqrt{\pi}} \\
& =\frac{\alpha}{8 \sqrt{\pi} \Delta k}\left\{(\Delta k+1)^{2}\left(1-{ }_{2} F_{2}\left[1,1 ; \frac{1}{2}, 2 ;-\frac{\alpha}{4 \Delta k}(\Delta k+1)^{2}\right]\right)-\left(1-{ }_{2} F_{2}\left[1,1 ; \frac{1}{2}, 2 ;-\frac{\alpha}{4 \Delta k}\right]\right)\right\}
\end{aligned}
$$

Con estas dos integrales podemos encontrar la solución de la integral $I_{3,1}$ D.84

$$
\begin{aligned}
& I_{3,1}=-\frac{k_{1}^{2} \tau \sqrt{\Delta k}}{m \gamma \epsilon\left(t_{0}\right) \alpha^{3 / 2}}\left[e ^ { \frac { \alpha } { 4 \Delta k } } \frac { \sqrt { \alpha } } { 4 \sqrt { \Delta k } } \left\{(\Delta k+1) e^{-\frac{\alpha}{4 \Delta k}(\Delta k+1)^{2}}+\frac{\sqrt{\Delta k} \sqrt{\pi}}{\sqrt{\alpha}} \operatorname{erf}\left(\frac{\sqrt{\alpha}}{2 \sqrt{\Delta k}}(\Delta k+1)\right)+e^{-\frac{\alpha}{4 \Delta k}}\right.\right. \\
& \left.-\frac{\sqrt{\Delta k} \sqrt{\pi}}{\sqrt{\alpha}} \operatorname{erf}\left(\frac{\sqrt{\alpha}}{2 \sqrt{\Delta k}}\right)\right\}-\frac{\sqrt{\alpha} \sqrt{\pi}}{2 \sqrt{\Delta k}} \operatorname{erfi}\left(\frac{\sqrt{\alpha}}{2 \sqrt{\Delta k}}\right) \frac{\sqrt{\alpha}}{4 \sqrt{\Delta k}}\left\{(\Delta k+1) e^{-\frac{\alpha}{4 \Delta k}(\Delta k+1)^{2}}\right. \\
& \left.+\frac{\sqrt{\Delta k} \sqrt{\pi}}{\sqrt{\alpha}} \operatorname{erf}\left(\frac{\sqrt{\alpha}}{2 \sqrt{\Delta k}}(\Delta k+1)\right)+e^{-\frac{\alpha}{4 \Delta k}}-\frac{\sqrt{\Delta k} \sqrt{\pi}}{\sqrt{\alpha}} \operatorname{erf}\left(\frac{\sqrt{\alpha}}{2 \sqrt{\Delta k}}\right)\right\}+\frac{\sqrt{\alpha} \sqrt{\pi}}{2 \sqrt{\Delta k}} \frac{\alpha}{8 \sqrt{\pi} \Delta k} \\
& \left.\left\{(\Delta k+1)^{2}\left(1-{ }_{2} F_{2}\left[1,1 ; \frac{1}{2}, 2 ;-\frac{\alpha}{4 \Delta k}(\Delta k+1)^{2}\right]\right)-\left(1-{ }_{2} F_{2}\left[1,1 ; \frac{1}{2}, 2 ;-\frac{\alpha}{4 \Delta k}\right]\right)\right\}\right] \\
& =-\frac{k_{1}^{2} \tau \sqrt{\Delta k}}{m \gamma \epsilon\left(t_{0}\right) \alpha^{3 / 2}}\left[e ^ { \frac { \alpha } { \Delta \Delta k } } \frac { \sqrt { \alpha } } { 4 \sqrt { \Delta k } } \left\{(\Delta k+1) e^{-\frac{\alpha}{4 \Delta k}(\Delta k+1)^{2}}+\frac{\sqrt{\Delta k} \sqrt{\pi}}{\sqrt{\alpha}} \operatorname{erf}\left(\frac{\sqrt{\alpha}}{2 \sqrt{\Delta k}}(\Delta k+1)\right)+e^{-\frac{\alpha}{4 \Delta k}}\right.\right. \\
& \left.-\frac{\sqrt{\Delta k} \sqrt{\pi}}{\sqrt{\alpha}} \operatorname{erf}\left(\frac{\sqrt{\alpha}}{2 \sqrt{\Delta k}}\right)\right\}-\frac{\alpha \sqrt{\pi}}{8 \Delta k} \operatorname{erfi}\left(\frac{\sqrt{\alpha}}{2 \sqrt{\Delta k}}\right)\left\{(\Delta k+1) e^{-\frac{\alpha}{4 \Delta k}(\Delta k+1)^{2}}\right. \\
& \left.+\frac{\sqrt{\Delta k} \sqrt{\pi}}{\sqrt{\alpha}} \operatorname{erf}\left(\frac{\sqrt{\alpha}}{2 \sqrt{\Delta k}}(\Delta k+1)\right)+e^{-\frac{\alpha}{4 \Delta k}}-\frac{\sqrt{\Delta k} \sqrt{\pi}}{\sqrt{\alpha}} \operatorname{erf}\left(\frac{\sqrt{\alpha}}{2 \sqrt{\Delta k}}\right)\right\}+\frac{\alpha^{3} / 2}{16 \Delta k^{3 / 2}} \\
& \left.\left\{(\Delta k+1)^{2}\left(1-{ }_{2} F_{2}\left[1,1 ; \frac{1}{2}, 2 ;-\frac{\alpha}{4 \Delta k}(\Delta k+1)^{2}\right]\right)-\left(1-{ }_{2} F_{2}\left[1,1 ; \frac{1}{2}, 2 ;-\frac{\alpha}{4 \Delta k}\right]\right)\right\}\right] \\
& \left.-\frac{\sqrt{\Delta k} \sqrt{\pi}}{\sqrt{\alpha}} \operatorname{erf}\left(\frac{\sqrt{\alpha}}{2 \sqrt{\Delta k}}\right)\right\}-\frac{\sqrt{\alpha} \sqrt{\pi}}{2 \sqrt{\Delta k}} \operatorname{erfi}\left(\frac{\sqrt{\alpha}}{2 \sqrt{\Delta k}}\right)\left\{(\Delta k+1) e^{-\frac{\alpha}{4 \Delta k}(\Delta k+1)^{2}}\right. \\
& \left.+\frac{\sqrt{\Delta k} \sqrt{\pi}}{\sqrt{\alpha}} \operatorname{erf}\left(\frac{\sqrt{\alpha}}{2 \sqrt{\Delta k}}(\Delta k+1)\right)+e^{-\frac{\alpha}{4 \Delta k}}-\frac{\sqrt{\Delta k} \sqrt{\pi}}{\sqrt{\alpha}} \operatorname{erf}\left(\frac{\sqrt{\alpha}}{2 \sqrt{\Delta k}}\right)\right\}+\frac{\alpha}{4 \Delta k} \\
& \left.\left\{(\Delta k+1)^{2}\left(1-{ }_{2} F_{2}\left[1,1 ; \frac{1}{2}, 2 ;-\frac{\alpha}{4 \Delta k}(\Delta k+1)^{2}\right]\right)-\left(1-{ }_{2} F_{2}\left[1,1 ; \frac{1}{2}, 2 ;-\frac{\alpha}{4 \Delta k}\right]\right)\right\}\right] \\
& =-\frac{k_{1}^{2} \tau}{4 m \gamma \epsilon\left(t_{0}\right) \alpha}\left[e ^ { \frac { \alpha } { 4 \Delta k } } \left\{(\Delta k+1) e^{-\frac{\alpha}{4 \Delta k}(\Delta k+1)^{2}}+\frac{\sqrt{\Delta k} \sqrt{\pi}}{\sqrt{\alpha}} \operatorname{erf}\left(\frac{\sqrt{\alpha}}{2 \sqrt{\Delta k}}(\Delta k+1)\right)+e^{-\frac{\alpha}{4 \Delta k}}\right.\right. \\
& \left.-\frac{\sqrt{\Delta k} \sqrt{\pi}}{\sqrt{\alpha}} \operatorname{erf}\left(\frac{\sqrt{\alpha}}{2 \sqrt{\Delta k}}\right)\right\}-\frac{\sqrt{\alpha} \sqrt{\pi}}{2 \sqrt{\Delta k}} \operatorname{erfi}\left(\frac{\sqrt{\alpha}}{2 \sqrt{\Delta k}}\right)\left\{(\Delta k+1) e^{-\frac{\alpha}{4 \Delta k}(\Delta k+1)^{2}}\right. \\
& \left.+\frac{\sqrt{\Delta k} \sqrt{\pi}}{\sqrt{\alpha}} \operatorname{erf}\left(\frac{\sqrt{\alpha}}{2 \sqrt{\Delta k}}(\Delta k+1)\right)+e^{-\frac{\alpha}{4 \Delta k}}-\frac{\sqrt{\Delta k} \sqrt{\pi}}{\sqrt{\alpha}} \operatorname{erf}\left(\frac{\sqrt{\alpha}}{2 \sqrt{\Delta k}}\right)\right\}+\frac{\alpha}{4 \Delta k} \\
& \left.\left\{(\Delta k+1)^{2}\left(1-{ }_{2} F_{2}\left[1,1 ; \frac{1}{2}, 2 ;-\frac{\alpha}{4 \Delta k}(\Delta k+1)^{2}\right]\right)-\left(1-{ }_{2} F_{2}\left[1,1 ; \frac{1}{2}, 2 ;-\frac{\alpha}{4 \Delta k}\right]\right)\right\}\right]
\end{aligned}
$$

Identificamos $\alpha$

$$
\begin{aligned}
& =-\frac{k_{1} \alpha}{4 \epsilon\left(t_{0}\right) \alpha}\left[e ^ { \frac { \alpha } { 4 \Delta k } } \left\{(\Delta k+1) e^{-\frac{\alpha}{4 \Delta k}(\Delta k+1)^{2}}+\frac{\sqrt{\Delta k} \sqrt{\pi}}{\sqrt{\alpha}} \operatorname{erf}\left(\frac{\sqrt{\alpha}}{2 \sqrt{\Delta k}}(\Delta k+1)\right)+e^{-\frac{\alpha}{4 \Delta k}}\right.\right. \\
& \left.-\frac{\sqrt{\Delta k} \sqrt{\pi}}{\sqrt{\alpha}} \operatorname{erf}\left(\frac{\sqrt{\alpha}}{2 \sqrt{\Delta k}}\right)\right\}-\frac{\sqrt{\alpha} \sqrt{\pi}}{2 \sqrt{\Delta k}} \operatorname{erfi}\left(\frac{\sqrt{\alpha}}{2 \sqrt{\Delta k}}\right)\left\{(\Delta k+1) e^{-\frac{\alpha}{4 \Delta k}(\Delta k+1)^{2}}\right.
\end{aligned}
$$




$$
\begin{aligned}
& \left.+\frac{\sqrt{\Delta k} \sqrt{\pi}}{\sqrt{\alpha}} \operatorname{erf}\left(\frac{\sqrt{\alpha}}{2 \sqrt{\Delta k}}(\Delta k+1)\right)+e^{-\frac{\alpha}{4 \Delta k}}-\frac{\sqrt{\Delta k} \sqrt{\pi}}{\sqrt{\alpha}} \operatorname{erf}\left(\frac{\sqrt{\alpha}}{2 \sqrt{\Delta k}}\right)\right\}+\frac{\alpha}{4 \Delta k} \\
& \left.\left\{(\Delta k+1)^{2}\left(1-{ }_{2} F_{2}\left[1,1 ; \frac{1}{2}, 2 ;-\frac{\alpha}{4 \Delta k}(\Delta k+1)^{2}\right]\right)-\left(1-{ }_{2} F_{2}\left[1,1 ; \frac{1}{2}, 2 ;-\frac{\alpha}{4 \Delta k}\right]\right)\right\}\right]
\end{aligned}
$$

La segunda integral $I_{3,2}$ es más sencilla y se puede resolver directamente

$$
\begin{aligned}
I_{3,2} & =\int_{\frac{2 \tau}{4}}^{\frac{3 \tau}{4}} \frac{q_{1} k_{1}\left(\frac{\Delta k}{\tau} 4\left(t-\frac{2 \tau}{4}\right)+1\right)}{2 \gamma^{2}} d t=\frac{q_{1} k_{1}}{2 \gamma^{2}}\left\{\int_{\frac{2 \tau}{4}}^{\frac{3 \tau}{4}} \frac{4 \Delta k t}{\tau} d t+\int_{\frac{2 \tau}{4}}^{\frac{3 \tau}{4}}(-2 \Delta k+1) d t\right\} \\
& =\frac{q_{1} k_{1}}{2 \gamma^{2}}\left\{\frac{2 \Delta k t^{2}}{\tau}+(-2 \Delta k+1) t\right\}_{\frac{2 \tau}{4}}^{\frac{3 \tau}{4}}=\frac{q_{1} k_{1}}{2 \gamma^{2}}\left\{\frac{2 \Delta k\left(\frac{3 \tau}{4}\right)^{2}}{\tau}+(-2 \Delta k+1) \frac{3 \tau}{4}-\frac{2 \Delta k\left(\frac{2 \tau}{4}\right)^{2}}{\tau}-(-2 \Delta k+1) \frac{2 \tau}{4}\right\} \\
& =\frac{q_{1} k_{1}}{2 \gamma^{2}}\left\{\frac{5 \Delta k \tau^{2}}{8 \tau}+(-2 \Delta k+1) \frac{\tau}{4}\right\}=\frac{q_{1} k_{1} \tau(\Delta k+2)}{16 \gamma^{2}}
\end{aligned}
$$

Multiplicamos y dividimos por $m$ y $k_{1}$ para identificar $\alpha$ y $\epsilon\left(t_{0}\right)=\frac{\gamma k_{1}}{m q_{1}}$

$$
I_{3,2}=\frac{q_{1} k_{1} \tau(\Delta k+2)}{16 \gamma^{2}} \frac{k_{1} m}{k_{1} m}=\frac{k_{1} \alpha(\Delta k+2)}{16 \epsilon\left(t_{0}\right)}=\frac{k_{1} \alpha(\Delta k+2)}{16 \varepsilon}
$$

La expresión del calor para este proceso es

$$
\begin{aligned}
& =-\frac{k_{1}}{4 \varepsilon}\left[e ^ { \frac { \alpha } { 4 \Delta k } } \left\{(\Delta k+1) e^{-\frac{\alpha}{4 \Delta k}(\Delta k+1)^{2}}+\frac{\sqrt{\Delta k} \sqrt{\pi}}{\sqrt{\alpha}} \operatorname{erf}\left(\frac{\sqrt{\alpha}}{2 \sqrt{\Delta k}}(\Delta k+1)\right)+e^{-\frac{\alpha}{4 \Delta k}}\right.\right. \\
& \left.-\frac{\sqrt{\Delta k} \sqrt{\pi}}{\sqrt{\alpha}} \operatorname{erf}\left(\frac{\sqrt{\alpha}}{2 \sqrt{\Delta k}}\right)\right\}-\frac{\sqrt{\alpha} \sqrt{\pi}}{2 \sqrt{\Delta k}} \operatorname{erfi}\left(\frac{\sqrt{\alpha}}{2 \sqrt{\Delta k}}\right)\left\{(\Delta k+1) e^{-\frac{\alpha}{4 \Delta k}(\Delta k+1)^{2}}\right. \\
& \left.+\frac{\sqrt{\Delta k} \sqrt{\pi}}{\sqrt{\alpha}} \operatorname{erf}\left(\frac{\sqrt{\alpha}}{2 \sqrt{\Delta k}}(\Delta k+1)\right)+e^{-\frac{\alpha}{4 \Delta k}}-\frac{\sqrt{\Delta k} \sqrt{\pi}}{\sqrt{\alpha}} \operatorname{erf}\left(\frac{\sqrt{\alpha}}{2 \sqrt{\Delta k}}\right)\right\}+\frac{\alpha}{4 \Delta k} \\
& \left.\left\{(\Delta k+1)^{2}\left(1-{ }_{2} F_{2}\left[1,1 ; \frac{1}{2}, 2 ;-\frac{\alpha}{4 \Delta k}(\Delta k+1)^{2}\right]\right)-\left(1-{ }_{2} F_{2}\left[1,1 ; \frac{1}{2}, 2 ;-\frac{\alpha}{4 \Delta k}\right]\right)\right\}\right]+\frac{k_{1} \alpha(\Delta k+2)}{16 \varepsilon} \\
& =\frac{k_{1}}{4 \varepsilon}\left[-e \frac{\alpha}{4 \Delta k}\left\{(\Delta k+1) e^{-\frac{\alpha}{4 \Delta k}(\Delta k+1)^{2}}+\frac{\sqrt{\Delta k} \sqrt{\pi}}{\sqrt{\alpha}} \operatorname{erf}\left(\frac{\sqrt{\alpha}}{2 \sqrt{\Delta k}}(\Delta k+1)\right)+e^{-\frac{\alpha}{4 \Delta k}}\right.\right. \\
& \left.-\frac{\sqrt{\Delta k} \sqrt{\pi}}{\sqrt{\alpha}} \operatorname{erf}\left(\frac{\sqrt{\alpha}}{2 \sqrt{\Delta k}}\right)\right\}+\frac{\sqrt{\alpha} \sqrt{\pi}}{2 \sqrt{\Delta k}} \operatorname{erfi}\left(\frac{\sqrt{\alpha}}{2 \sqrt{\Delta k}}\right)\left\{(\Delta k+1) e^{-\frac{\alpha}{4 \Delta k}(\Delta k+1)^{2}}\right. \\
& \left.+\frac{\sqrt{\Delta k} \sqrt{\pi}}{\sqrt{\alpha}} \operatorname{erf}\left(\frac{\sqrt{\alpha}}{2 \sqrt{\Delta k}}(\Delta k+1)\right)+e^{-\frac{\alpha}{4 \Delta k}}-\frac{\sqrt{\Delta k} \sqrt{\pi}}{\sqrt{\alpha}} \operatorname{erf}\left(\frac{\sqrt{\alpha}}{2 \sqrt{\Delta k}}\right)\right\}-\frac{\alpha}{4 \Delta k} \\
& \left.\left\{(\Delta k+1)^{2}\left(1-{ }_{2} F_{2}\left[1,1 ; \frac{1}{2}, 2 ;-\frac{\alpha}{4 \Delta k}(\Delta k+1)^{2}\right]\right)-\left(1-{ }_{2} F_{2}\left[1,1 ; \frac{1}{2}, 2 ;-\frac{\alpha}{4 \Delta k}\right]\right)\right\}+\frac{\alpha(\Delta k+2)}{4}\right]
\end{aligned}
$$

con el mismo análisis adimensional que hemos hecho en los otros procesos notamos que podemos escribir una expresión adimensional para el calor en este proceso $\left\langle\Delta Q_{3 \rightarrow 4}\right\rangle^{*}=\frac{\varepsilon\left\langle Q_{3 \rightarrow 4}\right\rangle}{k_{1}}$

$$
\begin{aligned}
\left\langle Q_{3 \rightarrow 4}\right\rangle^{*} & =-\frac{e^{\frac{\alpha}{4 \Delta k}}}{4}\left\{(\Delta k+1) e^{-\frac{\alpha}{4 \Delta k}(\Delta k+1)^{2}}+\frac{\sqrt{\Delta k} \sqrt{\pi}}{\sqrt{\alpha}} \operatorname{erf}\left(\frac{\sqrt{\alpha}}{2 \sqrt{\Delta k}}(\Delta k+1)\right)+e^{-\frac{\alpha}{4 \Delta k}}\right. \\
& \left.-\frac{\sqrt{\Delta k} \sqrt{\pi}}{\sqrt{\alpha}} \operatorname{erf}\left(\frac{\sqrt{\alpha}}{2 \sqrt{\Delta k}}\right)\right\}+\frac{\sqrt{\alpha} \sqrt{\pi}}{8 \sqrt{\Delta k}} \operatorname{erfi}\left(\frac{\sqrt{\alpha}}{2 \sqrt{\Delta k}}\right)\left\{(\Delta k+1) e^{-\frac{\alpha}{4 \Delta k}(\Delta k+1)^{2}}\right. \\
& \left.+\frac{\sqrt{\Delta k} \sqrt{\pi}}{\sqrt{\alpha}} \operatorname{erf}\left(\frac{\sqrt{\alpha}}{2 \sqrt{\Delta k}}(\Delta k+1)\right)+e^{-\frac{\alpha}{4 \Delta k}}-\frac{\sqrt{\Delta k} \sqrt{\pi}}{\sqrt{\alpha}} \operatorname{erf}\left(\frac{\sqrt{\alpha}}{2 \sqrt{\Delta k}}\right)\right\}-\frac{\alpha}{16 \Delta k} \\
& \left\{(\Delta k+1)^{2}\left(1-{ }_{2} F_{2}\left[1,1 ; \frac{1}{2}, 2 ;-\frac{\alpha}{4 \Delta k}(\Delta k+1)^{2}\right]\right)-\left(1-{ }_{2} F_{2}\left[1,1 ; \frac{1}{2}, 2 ;-\frac{\alpha}{4 \Delta k}\right]\right)\right\} \\
& +\frac{\alpha(\Delta k+2)}{16}
\end{aligned}
$$

Esta expresión depende únicamente de $\Delta k$, para analizarla damos un valor numérico a la diferencia de rigidez de la trampa.

$$
\Delta k=1
$$

De esta manera podemos encontrar el límite cuando $\alpha$ tiende a infinito

$$
\lim _{\alpha \rightarrow \infty}\left\langle Q_{3 \rightarrow 4}\right\rangle^{*}=-\frac{\log [2]}{4}
$$

Graficamos para determinar el comportamiento del calor cuando $\Delta k=1$ en función de $\alpha$ como se muestra en el capítulo 7 


\section{D.4. 4 a 1}

Este proceso es un cambio en la intensidad del ruido manteniendo la rigidez del potencial constante. Las escribimos en las funciones adimensionales $\Delta Q$ y $\Delta K$.

$$
\begin{aligned}
& k(t)=k_{2}=k_{1}(1+\Delta k) \\
& q(t)=\frac{q_{2}-q_{1}}{\tau} 4\left(t-\frac{3 \tau}{4}\right)+q_{1}=q_{1}\left(1+\Delta Q\left(-3+\frac{4 t}{\tau}\right)\right)
\end{aligned}
$$

La forma de $\epsilon(t)$ durante este proceso es

$$
\begin{aligned}
\frac{1}{\epsilon_{4 \rightarrow 1}(t)} & =\frac{1}{\epsilon\left(t_{0}\right)}\left[e^{\frac{2 \alpha t(1+\Delta k)\left(-1+\frac{3 \tau}{4 t}\right)}{\tau}}+\frac{t}{\alpha \tau(1+\Delta k)}\left[4 \alpha \Delta q(1+\Delta k)-\frac{\tau}{t}\left(-2 \Delta q\left(-1+e^{\frac{2 \alpha t(1+\Delta k)\left(-1+\frac{3 \tau}{4 t}\right)}{\tau}}\right)\right.\right.\right. \\
& \left.\left.\left.+\alpha(1+\Delta k)\left(-1+e^{\frac{2 \alpha t(1+\Delta k)\left(-1+\frac{3 \tau}{4 t}\right)}{\tau}}+3 \Delta q\right)\right)\right]\right]
\end{aligned}
$$

para encontrar el calor es necesario resolver las siguientes integrales

$$
\left\langle\Delta Q_{4 \rightarrow 1}\right\rangle=\underbrace{\int_{\frac{3 \tau}{4}}^{\tau}-\frac{k_{1}^{2}(1+\Delta k)^{2}}{2 m \gamma \epsilon_{4 \rightarrow 1}(t)}}_{I_{4,1}} d t+\underbrace{\int_{\frac{3 \tau}{4}}^{\tau} \frac{k_{1}(1+\Delta k) q_{1}\left(1+\Delta q\left(-3+\frac{4 t}{\tau}\right)\right)}{2 \gamma^{2}} d t}_{I_{4,2}}
$$

comenzamos resolviendo la integral $I_{4,1}$, es una integral relativamente sencilla ya que el exponente de la exponencial es lineal en el tiempo, su integral no nos lleva a funciones de error

$$
\begin{aligned}
I_{4,1} & =\int_{\frac{3 \tau}{4}}^{\tau}-\frac{k_{1}^{2}(1+\Delta k)^{2}}{2 m \gamma \epsilon_{4 \rightarrow 1}(t)} d t \\
& =-\frac{k_{1}^{2}(1+\Delta k)^{2}}{2 m \gamma \epsilon\left(t_{0}\right)} \int_{\frac{3 \tau}{4}}^{\tau}\left[e^{\frac{2 \alpha t(1+\Delta k)\left(-1+\frac{3 \tau}{4 t}\right)}{\tau}}+\frac{t}{\alpha \tau(1+\Delta k)}\left[4 \alpha \Delta q(1+\Delta k)-\frac{\tau}{t}\left(-2 \Delta q\left(-1+e^{\frac{2 \alpha t(1+\Delta k)\left(-1+\frac{3 \tau}{4 t}\right)}{\tau}}\right)\right.\right.\right. \\
& \left.\left.\left.+\alpha(1+\Delta k)\left(-1+e^{\frac{2 \alpha t(1+\Delta k)\left(-1+\frac{3 \tau}{4 t}\right)}{\tau}}+3 \Delta q\right)\right)\right]\right] d t \\
& =-\frac{k_{1}^{2}(1+\Delta k)^{2}}{2 m \gamma \epsilon\left(t_{0}\right)} \frac{\tau\left(-8 \Delta q e^{-\frac{\alpha}{2}(1+\Delta k)}+8 \Delta q-4 \alpha \Delta q(1+\Delta k)+\alpha^{2}(1+\Delta k)^{2}(2+\Delta q)\right)}{8 \alpha^{2}(1+\Delta k)^{2}}
\end{aligned}
$$

identificamos $\alpha$

$$
\begin{aligned}
& =-\frac{k_{1} \alpha}{16 \epsilon\left(t_{0}\right)} \frac{\left(-8 \Delta q e^{-\frac{\alpha}{2}(1+\Delta k)}+8 \Delta q-4 \alpha \Delta q(1+\Delta k)+\alpha^{2}(1+\Delta k)^{2}(2+\Delta q)\right)}{\alpha^{2}} \\
& =-\frac{k_{1}}{16 \alpha \epsilon\left(t_{0}\right)}\left(-8 \Delta q e^{-\frac{\alpha}{2}(1+\Delta k)}+8 \Delta q-4 \alpha \Delta q(1+\Delta k)+\alpha^{2}(1+\Delta k)^{2}(2+\Delta q)\right)
\end{aligned}
$$

La segunda integral $I_{4,2}$ es más sencilla

$$
\begin{aligned}
I_{4,2}= & \frac{k_{1} q_{1}(1+\Delta k)}{2 \gamma^{2}} \int_{\frac{3 \tau}{4}}^{\tau}\left(1+\Delta q\left(-3+\frac{4 t}{\tau}\right)\right) d t=\frac{k_{1} q_{1}(1+\Delta k)}{2 \gamma^{2}} \int_{\frac{3 \tau}{4}}^{\tau}\left(1-3 \Delta q+\frac{4 t}{\tau} \Delta q\right) d t \\
& =\frac{k_{1} q_{1}(1+\Delta k)}{2 \gamma^{2}}\left(t-3 t \Delta q+\frac{2 \Delta q t^{2}}{\tau}\right)_{\frac{3 \tau}{4}}^{\tau}=\frac{k_{1} q_{1}(1+\Delta k)}{2 \gamma^{2}}\left(\frac{\tau}{4}+\frac{\Delta q \tau}{8}\right) \\
& =\frac{k_{1} q_{1} \tau(1+\Delta k)}{16 \gamma^{2}}(2+\Delta q) \\
& \text { Multiplicamos y dividimos por } m k_{2} \text { para identificar } \alpha \mathrm{y} \epsilon\left(t_{0}\right)=\frac{\gamma k_{2}}{m q_{1}} \\
& =\frac{k_{1} q_{1} m k_{2} \tau(1+\Delta k)}{16 m k_{2} \gamma^{2}}(2+\Delta q)=\frac{k_{1} \alpha(1+\Delta k)^{2}(2+\Delta k)}{16 \epsilon\left(t_{0}\right)}
\end{aligned}
$$

La expresión para el calor queda de la siguiente manera

$$
\begin{aligned}
\left\langle\Delta Q_{4 \rightarrow 1}\right\rangle & =-\frac{k_{1}}{16 \alpha \epsilon\left(t_{0}\right)}\left(-8 \Delta q e^{-\frac{\alpha}{2}(1+\Delta k)}+8 \Delta q-4 \alpha \Delta q(1+\Delta k)+\alpha^{2}(1+\Delta k)^{2}(2+\Delta q)\right) \\
& +\frac{k_{1} \alpha(1+\Delta k)^{2}(2+\Delta k)}{16 \epsilon\left(t_{0}\right)}
\end{aligned}
$$




$$
\begin{aligned}
& =\frac{k_{1}}{16 \epsilon\left(t_{0}\right)}\left[+\frac{8 \Delta q e^{-\frac{\alpha}{2}(1+\Delta k)}+8 \Delta q}{\alpha}+4 \Delta q(1+\Delta k)-\alpha(1+\Delta k)^{2}(2+\Delta q)\right. \\
& \left.+\alpha(1+\Delta k)^{2}(2+\Delta k)\right] \\
& =\frac{k_{1}}{16 \epsilon\left(t_{0}\right)}\left[\frac{8 \Delta q\left(e^{-\frac{\alpha}{2}(1+\Delta k)}+1\right)}{\alpha}+4 \Delta q(1+\Delta k)\right] \\
& \text { pero } \epsilon\left(t_{0}\right)=\frac{\gamma k_{2}}{m q_{1}}=\frac{\gamma k_{1}(1+\Delta k)}{m q_{1}}=\varepsilon(1+\Delta k) \\
& =\frac{k_{1}}{16 \varepsilon(1+\Delta k)}\left[\frac{8 \Delta q\left(e^{-\frac{\alpha}{2}(1+\Delta k)}+1\right)}{\alpha}+4 \Delta q(1+\Delta k)\right]
\end{aligned}
$$

Podemos reescribirlo como un calor adimensional $\frac{\varepsilon\left\langle\Delta Q_{4 \rightarrow 1}\right\rangle}{k_{1}}$

$$
\left\langle\Delta Q_{4 \rightarrow 1}\right\rangle^{*}=\frac{\Delta q\left(e^{-\frac{\alpha}{2}(1+\Delta k)}+1\right)}{2 \alpha(1+\Delta k)}+\frac{\Delta q}{4}
$$

El límite cuando $\alpha$ tiende a infinito

$$
\lim _{\alpha \rightarrow \infty}\left\langle\Delta Q_{4 \rightarrow 1}\right\rangle^{*}=\frac{\Delta q}{4}
$$

Dando valor a $\Delta q=1$ y $\Delta k=1$ podemos graficar obteniendo el comportamiento mostrado en el capítulo 7

Durante el ciclo en los cuatro procesos existe una transferencia de energía entre el medio y la partícula, al que llamamos calor, el signo nos indica si el calor está siendo absorbido por la partícula o si está siendo liberado al medio. En nuestra convención de signos, calor positivo indica que el sistema está recibiendo calor del medio, calor negativo indica lo contrario. durante el proceso de 1 a 2 , cuando disminuye la rigidez manteniendo una intensidad de ruido constante y el proceso de 4 a 1 cuando la intensidad del ruido aumenta con una rigidez de la trampa constante tenemos un calor positivo, esto implica que durante estos procesos se está absorbiendo calor, durante los otros dos procesos se está liberando calor al medio. tomando en cuenta solo los límites cuando $\alpha$ es muy grande y los valores otorgados a $\Delta k$ y $\Delta q$ podemos ver que al sumar los calores $\left\langle\Delta Q_{3 \rightarrow 3}\right\rangle^{*}$ y $\left\langle\Delta Q_{4 \rightarrow 1}\right\rangle^{*}$ se eliminan sin importar el valor de las $k$ y $q$ y al final del proceso

$$
\left\langle\Delta Q_{1 \rightarrow 2}\right\rangle^{*}+\left\langle\Delta Q_{2 \rightarrow 3}\right\rangle^{*}+\left\langle\Delta Q_{3 \rightarrow 4}\right\rangle^{*}+\left\langle\Delta Q_{4 \rightarrow 1}\right\rangle^{*}=-\frac{\log [2]}{4}+\frac{\log [2]}{2}=\frac{\log [2]}{4}
$$

El calor final es positivo, esto indica que el sistema está absorbiendo calor. Complementando con el trabajo, el sistema absorbe calor del medio y se convierte en trabajo. 



\section{Apéndice $\mathrm{E}$}

\section{Trabajo en el ciclo}

El trabajo que se realiza durante un proceso termodinámico sobre la partícula está dado por

$$
\Delta W=\frac{\partial U(x, k)}{\partial k} \circ d k=\frac{x(t)^{2}}{2} \frac{d k(t)}{d t} d t
$$

Debido a que el sistema es una partícula Browniana entonces la posición es una variable aleatoria y el proceso es estocástico, la solución de esta ecuación tendrá un valor diferente en cada realización del proceso pero el promedio sobre el ensamble está dado por

$$
\langle\Delta W(x, t)\rangle=\int_{t_{0}}^{t} \frac{1}{4 \epsilon(t)} \frac{d k(t)}{d t} d t
$$

ya hemos encontrado que $\frac{1}{\epsilon(t)}$ es diferente para cada proceso

\section{E.1. 1 a 2}

El primer proceso se realiza con el protocolo

$$
\begin{aligned}
& k(t)=\frac{k_{1}-k_{2}}{\tau} 4 t+k_{2} \\
& q(t)=q_{2}
\end{aligned}
$$

es un cambio en la rigidez del potencial mientras se mantiene constante el ruido. Para encontrar el promedio del trabajo realizado es necesario encontrar el cambio en la rigidez del potencial

$$
\dot{k}(t)=\frac{d k(t)}{d t}=\frac{k_{1}-k_{1}}{\tau} 4
$$

y la forma de $\epsilon(t)$

$$
\begin{aligned}
\frac{1}{\epsilon_{1 \rightarrow 2}} & =\frac{1}{\epsilon\left(t_{0}\right)}\left(e^{\frac{2 \alpha t}{\tau}\left[2 \Delta k \frac{t}{\tau}-(\Delta k+1)\right]}\right. \\
& \left.+\frac{\sqrt{\alpha}(1+\Delta k) \sqrt{\pi} e^{\frac{\alpha}{4 \Delta k}\left(-4 \Delta k \frac{t}{\tau}+(\Delta k+1)\right.}}{2 \sqrt{\Delta k}}\left(\operatorname{erf}\left[\frac{\sqrt{\alpha}(\Delta k+1)}{2 \sqrt{\Delta k}}\right]-\operatorname{erf}\left[\frac{\sqrt{\alpha}\left(-4 \Delta k \frac{t}{\tau}+(\Delta k+1)\right)}{2 \sqrt{\Delta k}}\right]\right)\right)
\end{aligned}
$$

De manera que debemos de resolver la integral

$$
\begin{aligned}
& \int_{t=0}^{t=\frac{\tau}{4}}\left\{[ \frac { k _ { 1 } - k _ { 2 } } { \epsilon ( t _ { 0 } ) \tau } ] \quad \left[e^{\frac{2 \alpha t}{\tau}\left[2 \Delta k \frac{t}{\tau}-(\Delta k+1)\right]}\right.\right. \\
& \left.\left.+\frac{\sqrt{\alpha}(1+\Delta k) \sqrt{\pi} e^{\frac{\alpha}{4 \Delta k}\left(-4 \Delta k \frac{t}{\tau}+(\Delta k+1)\right.}}{2 \sqrt{\Delta k}}\left(\operatorname{erf}\left[\frac{\sqrt{\alpha}(\Delta k+1)}{2 \sqrt{\Delta k}}\right]-\operatorname{erf}\left[\frac{\sqrt{\alpha}\left(-4 \Delta k \frac{t}{\tau}+(\Delta k+1)\right)}{2 \sqrt{\Delta k}}\right]\right)\right]\right\} d t \\
& =\left[\frac{k_{1}-k_{2}}{\epsilon\left(t_{0}\right) \tau}\right] \int_{t=0}^{t=\frac{\tau}{4}}\{\underbrace{e^{\frac{2 \alpha t^{2}}{\tau^{2}}\left(2 \Delta k-(\Delta k+1) \frac{\tau}{t}\right)}}_{I_{1,1}}
\end{aligned}
$$




$$
+\underbrace{\frac{\sqrt{\alpha}(1+\Delta k) \sqrt{\pi} e^{\frac{\alpha t^{2}}{4 \Delta k \tau^{2}}\left(-4 \Delta k+(\Delta k+1) \frac{\tau}{t}\right)^{2}}}{2 \sqrt{\Delta k}}\left(\operatorname{erf}\left[\frac{\sqrt{\alpha}(\Delta k+1)}{2 \sqrt{\Delta k}}\right]-\operatorname{erf}\left[\frac{\sqrt{\alpha} t\left(-4 \Delta k+(\Delta k+1) \frac{\tau}{t}\right)}{2 \tau \sqrt{\Delta k}}\right]\right)}_{I_{1,2}}\} d t
$$

Podemos separar la integral en dos secciones

$$
\begin{aligned}
& I_{1.1}=\int_{t_{0}}^{t=\frac{\tau}{4}} e^{\frac{2 \alpha t^{2}}{\tau^{2}}\left(2 \Delta k-(\Delta k+1) \frac{\tau}{t}\right)} d t \\
& I_{1.2}=\int_{t_{0}}^{t=\frac{\tau}{4}} \frac{\sqrt{\alpha}(1+\Delta k) \sqrt{\pi} e^{\frac{\alpha t^{2}}{4 \Delta k \tau^{2}}\left(-4 \Delta k+(\Delta k+1) \frac{\tau}{t}\right)^{2}}}{2 \sqrt{\Delta k}}\left(\operatorname{erf}\left[\frac{\sqrt{\alpha}(\Delta k+1)}{2 \sqrt{\Delta k}}\right]-\operatorname{erf}\left[\frac{\sqrt{\alpha} t\left(-4 \Delta k+(\Delta k+1) \frac{\tau}{t}\right)}{2 \tau \sqrt{\Delta k}}\right]\right) d t
\end{aligned}
$$

la integral $I_{1.1}$ puede resolverse si reescribimos la forma del exponente como un cuadrado perfecto

$$
\frac{2 \alpha t^{2}}{\tau^{2}}\left(2 \Delta k-(\Delta k+1) \frac{\tau}{t}\right)=\frac{\alpha}{4 \Delta k}\left(\frac{-4 \Delta k t}{\tau}+(\Delta k+1)\right)^{2}-\frac{\alpha}{4 \Delta k}(\Delta k+1)^{2}
$$

$\mathrm{Al}$ escribirlo de esta manera tenemos una integral con forma Gaussiana pero que se encuentra limitada, usamos la propiedad de las funciones de error

$$
\operatorname{erfi}(x)=\frac{2}{\sqrt{\pi}} \int_{0}^{x} e^{t^{2}} d t
$$

para determinar la solución, la cual queda de la siguiente forma

$$
I_{1,1}=-\frac{\sqrt{\pi} \tau e^{-\frac{\alpha(\Delta k+1)^{2}}{4 \Delta k}}\left(\operatorname{erfi}\left[\frac{\sqrt{\alpha}}{2 \sqrt{\Delta k}}\right]-\operatorname{erfi}\left[\frac{\sqrt{\alpha}(1+\Delta k)}{2 \sqrt{\Delta k}}\right]\right)}{4 \sqrt{\alpha} \sqrt{\Delta k}}
$$

Nos falta encontrar la otra integral $I_{1,2}$, esta es una integral más complicada debido a que se tienen que integrar las funciones de error, podemos separarla en dos integrales

$$
\begin{aligned}
I_{1,2} & =\frac{\sqrt{\alpha} \sqrt{\pi}(1+\Delta k)}{2 \sqrt{\Delta k}} \operatorname{erf}\left[\frac{\sqrt{\alpha}(\Delta k+1)}{2 \sqrt{\Delta k}}\right] \int_{t_{0}}^{t=\frac{\tau}{4}} e^{\frac{\alpha t^{2}}{4 \Delta k \tau^{2}}\left(-4 \Delta k+(\Delta k+1) \frac{\tau}{t}\right)^{2}} d t \\
& -\frac{\sqrt{\alpha} \sqrt{\pi}(1+\Delta k)}{2 \sqrt{\Delta k}} \int_{t_{0}}^{t=\frac{\tau}{4}} e^{\frac{\alpha t^{2}}{4 \Delta k \tau^{2}}\left(-4 \Delta k+(\Delta k+1) \frac{\tau}{t}\right)^{2}} \operatorname{erf}\left[\frac{\sqrt{\alpha} t\left(-4 \Delta k+(\Delta k+1) \frac{\tau}{t}\right)}{2 \tau \sqrt{\Delta k}}\right] d t
\end{aligned}
$$

La primera parte de la integral es la integral de una Gaussiana compleja por lo que se usa la función de error compleja

$$
\begin{aligned}
& \frac{\sqrt{\alpha} \sqrt{\pi}(1+\Delta k)}{2 \sqrt{\Delta k}} \operatorname{erf}\left[\frac{\sqrt{\alpha}(\Delta k+1)}{2 \sqrt{\Delta k}}\right] \int_{t_{0}}^{t=\frac{\tau}{4}} e^{\frac{\alpha t^{2}}{4 \Delta k \tau^{2}}\left(-4 \Delta k+(\Delta k+1) \frac{\tau}{t}\right)^{2}} d t \\
& =\frac{\pi(1+\Delta k) \tau}{8 \Delta k} \operatorname{erf}\left[\frac{\sqrt{\alpha}(\Delta k+1)}{2 \sqrt{\Delta k}}\right]\left(\operatorname{erfi}\left[\frac{\sqrt{\alpha}(1+\Delta k)}{2 \sqrt{\Delta k}}\right]-\operatorname{erfi}\left[\frac{\sqrt{\alpha}}{2 \sqrt{\Delta k}}\right]\right)
\end{aligned}
$$

Para la segunda parte de la integral hacemos un cambio de variable

$$
u=\frac{\sqrt{\alpha}}{2 \tau \sqrt{\Delta k}}(-4 \Delta k t+(\Delta k+1) \tau)
$$

De manera que podemos reescribir

$$
\begin{aligned}
& \frac{\sqrt{\alpha} \sqrt{\pi}(1+\Delta k)}{2 \sqrt{\Delta k}} \int_{t_{0}}^{t=\frac{\tau}{4}} e^{\frac{\alpha t^{2}}{4 \Delta k \tau^{2}}\left(-4 \Delta k+(\Delta k+1) \frac{\tau}{t}\right)^{2}} \operatorname{erf}\left[\frac{\sqrt{\alpha} t\left(-4 \Delta k+(\Delta k+1) \frac{\tau}{t}\right)}{2 \tau \sqrt{\Delta k}}\right] d t \\
& =\frac{\sqrt{\pi} \tau(1+\Delta k)}{4 \Delta k} \int_{u\left(t_{0}\right)}^{u(\tau / 4)} e^{u^{2}} \operatorname{erf}(u) d u
\end{aligned}
$$

El resultado de esta integral es

$$
\int_{u\left(t_{0}\right)}^{u(\tau / 4)} e^{u^{2}} \operatorname{erf}(u) d u=\frac{1}{\sqrt{\pi}}\left(-u_{0}^{2} F\left[(1,1),\left(\frac{3}{2}, 2\right), u_{0}^{2}\right]+u_{f} F\left[(1,1),\left(\frac{3}{2}, 2\right), u_{f}^{2}\right]\right)
$$

Por lo que

$$
=-\frac{\sqrt{\pi} \tau(1+\Delta k)}{4 \Delta k} \int_{u\left(t_{0}\right)}^{u(\tau / 4)} e^{u^{2}} \operatorname{erf}(u) d u
$$




$$
=-\frac{\alpha \tau(1+\Delta k)}{(4 \Delta k)^{2}}\left(F\left[(1,1),\left(\frac{3}{2}, 2\right), \frac{\alpha}{4 \Delta k}\right]-(\Delta k+1)^{2} F\left[(1,1),\left(\frac{3}{2}, 2\right), \frac{\alpha(1+\Delta k)^{2}}{4 \Delta k}\right]\right)
$$

Uniendo las dos integrales que conforman la solución de $I_{1,2}$ llegamos a la expresión

$$
\begin{aligned}
I_{1,2} & =\frac{\sqrt{\pi} \tau(1+\Delta k)}{8 \Delta k}\left\{\sqrt{\pi} \operatorname{erf}\left[\frac{\sqrt{\alpha}(\Delta k+1)}{2 \sqrt{\Delta k}}\right]\left(\operatorname{erfi}\left[\frac{\sqrt{\alpha}(1+\Delta k)}{2 \sqrt{\Delta k}}\right]-\operatorname{erfi}\left[\frac{\sqrt{\alpha}}{2 \sqrt{\Delta k}}\right]\right)\right. \\
& \left.+\frac{\alpha}{2 \Delta k \sqrt{\pi}}\left(F\left[(1,1),\left(\frac{3}{2}, 2\right), \frac{\alpha}{4 \Delta k}\right]-(\Delta k+1)^{2} F\left[(1,1),\left(\frac{3}{2}, 2\right), \frac{\alpha(1+\Delta k)^{2}}{4 \Delta k}\right]\right)\right\}
\end{aligned}
$$

Con las solución de las integrales E.12 y E.18 solo hace falta sumarlas y multiplicar por $\frac{k_{1}-k_{2}}{\epsilon\left(t_{0}\right) \tau}$ para que tengamos de manera completa la expresión del trabajo. Podemos reescribir $\frac{k_{1}-k_{2}}{\epsilon\left(t_{0}\right) \tau}$ de manera adimensional. Tomamos en cuenta que durante este proceso la condición inicial está dada por

$$
\epsilon\left(t_{0}\right)=\frac{\gamma k_{2}}{m q_{2}}=\frac{\gamma k_{1}(1+\Delta k)}{m q 1(\Delta q)}=\frac{\varepsilon(1+\Delta k)}{(\Delta q)}
$$

en donde $\varepsilon=\frac{k_{1} \gamma}{m q_{1}}$ es una constante, de esta manera podemos reescribir

$$
\frac{k_{1}-k_{2}}{\epsilon\left(t_{0}\right) \tau}=-\frac{k_{1} \Delta k(1+\Delta q)}{\tau \varepsilon(1+\Delta k)}
$$

y la multiplicamos a las integrales para encontrar la expresión del trabajo promedio

$$
\begin{aligned}
\left\langle\Delta W_{1 \rightarrow 2}(x, t)\right\rangle & =-\frac{k 1 \sqrt{\pi} \sqrt{\Delta k}(1+\Delta q) e^{-\frac{\alpha(\Delta k+1)^{2}}{4 \Delta k}}}{4 \sqrt{\alpha} \varepsilon(1+\Delta k)}\left\{-\operatorname{erfi}\left[\frac{\sqrt{\alpha}}{2 \sqrt{\Delta k}}\right]+\operatorname{erfi}\left[\frac{\sqrt{\alpha}(1+\Delta k)}{2 \sqrt{\Delta k}}\right]\right. \\
& +\frac{\sqrt{\alpha}(1+\Delta k) e^{\frac{\alpha(\Delta k+1)^{2}}{4 \Delta k}}}{4 \sqrt{\pi} \Delta k^{3 / 2}}\left(2 \pi \Delta \operatorname{kerf}\left[\frac{\alpha(\Delta k+1)}{2 \sqrt{\Delta k}}\right]\left(\operatorname{erfi}\left[\frac{\sqrt{\alpha}(1+\Delta k)}{2 \sqrt{\Delta k}}\right]-\operatorname{erfi}\left[\frac{\sqrt{\alpha}}{2 \sqrt{\Delta k}}\right]\right)\right) \\
& \left.+\alpha F\left[(1,1),\left(\frac{3}{2}, 2\right), \frac{\alpha}{4 \Delta k}\right]-\alpha(\Delta k+1)^{2} F\left[(1,1),\left(\frac{3}{2}, 2\right), \frac{\alpha(1+\Delta k)^{2}}{4 \Delta k}\right]\right\}
\end{aligned}
$$

Las unidades del trabajo en el sistema internacional son $[W]=J=\frac{K g m^{2}}{s^{2}}$ del lado derecho de la expresión las únicas variables con dimensiones que restan son $k 1=\frac{K g}{s^{2}}$ y $\varepsilon=\frac{1}{m^{2}}$ al escribirlas de como está en la ecuación obtenemos las unidades del trabajo $\frac{k_{1}}{\varepsilon}=\frac{K g m^{2}}{s^{2}}$, por lo tanto podemos escribir un trabajo adimensional $\langle W\rangle^{*}=\frac{\varepsilon\langle W\rangle}{k_{1}}$ Entonces el trabajo adimensional para el primer proceso es

$$
\begin{aligned}
\left\langle\Delta W_{1 \rightarrow 2}(x, t)\right\rangle^{*} & =-\frac{\sqrt{\pi} \sqrt{\Delta k}(1+\Delta q) e^{-\frac{\alpha(\Delta k+1)^{2}}{4 \Delta k}}}{4 \sqrt{\alpha}(1+\Delta k)}\left\{-\operatorname{erfi}\left[\frac{\sqrt{\alpha}}{2 \sqrt{\Delta k}}\right]+\operatorname{erfi}\left[\frac{\sqrt{\alpha}(1+\Delta k)}{2 \sqrt{\Delta k}}\right]\right. \\
& +\frac{\sqrt{\alpha}(1+\Delta k) e^{\frac{\alpha(\Delta k+1)^{2}}{4 \Delta k}}}{4 \sqrt{\pi} \Delta k^{3 / 2}}\left(2 \pi \Delta \operatorname{kerf}\left[\frac{\alpha(\Delta k+1)}{2 \sqrt{\Delta k}}\right]\left(\operatorname{erfi}\left[\frac{\sqrt{\alpha}(1+\Delta k)}{2 \sqrt{\Delta k}}\right]-\operatorname{erfi}\left[\frac{\sqrt{\alpha}}{2 \sqrt{\Delta k}}\right]\right)\right) \\
& \left.+\alpha F\left[(1,1),\left(\frac{3}{2}, 2\right), \frac{\alpha}{4 \Delta k}\right]-\alpha(\Delta k+1)^{2} F\left[(1,1),\left(\frac{3}{2}, 2\right), \frac{\alpha(1+\Delta k)^{2}}{4 \Delta k}\right]\right\}
\end{aligned}
$$

Este trabajo solo depende del cambio de la rigidez del potencial $\Delta k$, del cambio en la intensidad del ruido $\Delta q$ y de la variable adimensional $\alpha=\frac{k_{1} \tau}{m \gamma}$.

Damos valores a las varables adimensionales $\Delta k=1$ y $\Delta q=1$ para graficar el trabajo con respecto a $\alpha$. Lo primero que nos interesa encontrar es el límite cuando alpha tiende a infinito

$$
\lim _{\alpha \rightarrow \infty}\left\langle\Delta W_{1 \rightarrow 2}(x, t)\right\rangle^{*}=-\frac{\log [2]}{2}
$$

Al graficar encontramos el comportamiento mostrado en el capítulo 7

\section{E.2. 2 a 3}

Este proceso es un cambio en la intensidad del ruido manteniendo la rigidez del potencial constante,

$$
\frac{d k(t)}{d t}=0
$$

ya que el trabajo depende del cambio en el potencial, durante este proceso no hay trabajo

$$
\langle W(x, t)\rangle=0
$$




\section{E.3. 3 a 4}

Este proceso es un cambio en la rigidez del potencial, mientras se mantiene un ruido constante, el protocolo es

$$
\begin{aligned}
& k(t)=\frac{k_{2}-k_{1}}{\tau} 4\left(t-\frac{2 \tau}{4}\right)+k_{1} \\
& q(t)=q_{1}
\end{aligned}
$$

Para encontrar el promedio del trabajo realizado es necesario encontrar el cambio en la rigidez del potencial

$$
\dot{k}(t)=\frac{d k(t)}{d t}=\frac{k_{2}-k_{1}}{\tau} 4
$$

la forma de $\epsilon(t)$ durante este proceso es el siguiente

$$
\begin{aligned}
\frac{1}{\epsilon_{3 \rightarrow 4}(t)} & =\frac{1}{\epsilon\left(t_{0}\right)}\left(e^{-\frac{\alpha t^{2}\left(2-\frac{\tau}{t}\right)\left(2 \Delta k+\frac{\tau}{t}-\Delta k \frac{\tau}{t}\right)}{\tau^{2}}}\right. \\
& \left.-\frac{\sqrt{\alpha} \sqrt{\pi} e^{-\frac{\alpha t^{2}}{4 \Delta k \tau^{2}}\left(4 \Delta k-2 \Delta k \frac{\tau}{t}+\frac{\tau}{t}\right)^{2}}}{2 \sqrt{\Delta k}}\left[\operatorname{erfi}\left(\frac{\sqrt{\alpha}}{2 \sqrt{\Delta k}}\right)-\operatorname{erfi}\left(\frac{\sqrt{\alpha} t\left(4 \Delta k+\frac{\tau}{t}-2 \Delta k \frac{\tau}{t}\right)}{2 \tau \sqrt{\Delta k}}\right)\right]\right)
\end{aligned}
$$

La integral a resolver es la siguiente

$$
\begin{aligned}
& \int_{t=\frac{\tau}{4}}^{t=\frac{2 \tau}{4}} \frac{1}{4 \epsilon(t)}\left[\frac{k_{2}-k_{1}}{\tau} 4\right]\left[e^{-\frac{\alpha t^{2}\left(2-\frac{\tau}{t}\right)\left(2 \Delta k+\frac{\tau}{t}-\Delta k \frac{\tau}{t}\right)}{\tau^{2}}}\right. \\
& \left.-\frac{\sqrt{\alpha} \sqrt{\pi} e^{-\frac{\alpha t^{2}}{4 \Delta k \tau^{2}}\left(4 \Delta k-2 \Delta k \frac{\tau}{t}+\frac{\tau}{t}\right)^{2}}}{2 \sqrt{\Delta k}}\left[\operatorname{erfi}\left(\frac{\sqrt{\alpha}}{2 \sqrt{\Delta k}}\right)-\operatorname{erfi}\left(\frac{\sqrt{\alpha} t\left(4 \Delta k+\frac{\tau}{t}-2 \Delta k \frac{\tau}{t}\right)}{2 \tau \sqrt{\Delta k}}\right)\right]\right] d t
\end{aligned}
$$

La solución de esta integral es muy parecida a la realizada en el proceso de $1 a 2$ el resultado es el siguiente

$$
\begin{aligned}
\left\langle W_{3 \rightarrow 4}\right\rangle & =\frac{\sqrt{\pi}\left(k_{1}-k_{2}\right)}{4 \sqrt{\alpha} \sqrt{\Delta k} \epsilon_{0}}\left\{e^{\frac{\alpha}{4 \Delta k}}\left(\operatorname{erf}\left[\frac{\sqrt{\alpha}}{2 \sqrt{\Delta k}}\right]-\operatorname{erf}\left[\frac{\sqrt{\alpha}(1+\Delta k)}{2 \sqrt{\Delta k}}\right]\right)\right. \\
& -\frac{2 \alpha}{8 \sqrt{\pi} \sqrt{\alpha} \Delta k^{3 / 2}}\left(2 \pi \Delta k \quad \operatorname{erf}\left[\frac{\sqrt{\alpha}}{2 \sqrt{\Delta k}}\right] \operatorname{erfi}\left[\frac{\sqrt{\alpha}}{2 \sqrt{\Delta k}}\right]-\alpha F\left[(1,1),\left(\frac{3}{2}, 2\right),-\frac{\alpha}{4 \Delta k}\right]\right. \\
& \left.\left.-2 \pi \Delta k \quad \operatorname{erf}\left[\frac{\sqrt{\alpha}(1+\Delta k)}{2 \sqrt{\Delta k}}\right] \operatorname{erfi}\left[\frac{\sqrt{\alpha}}{2 \sqrt{\Delta k}}\right]+\alpha(1+\Delta k)^{2} F\left[(1,1),\left(\frac{3}{2}, 2\right),-\frac{\alpha(1+\Delta k)^{2}}{4 \Delta k}\right]\right)\right\}
\end{aligned}
$$

pero $k_{2}-k_{1}=k_{1} \Delta k$ y $\epsilon\left(t_{0}\right)=\frac{\gamma k_{1}}{m q_{1}}=\varepsilon$ de esta manera

$$
\begin{aligned}
\left\langle W_{3 \rightarrow 4}\right\rangle & =-\frac{\sqrt{\pi} k_{1} \Delta k}{4 \sqrt{\alpha} \sqrt{\Delta k} \varepsilon}\left\{e^{\frac{\alpha}{4 \Delta k}}\left(\operatorname{erf}\left[\frac{\sqrt{\alpha}}{2 \sqrt{\Delta k}}\right]-\operatorname{erf}\left[\frac{\sqrt{\alpha}(1+\Delta k)}{2 \sqrt{\Delta k}}\right]\right)\right. \\
& -\frac{2 \alpha}{8 \sqrt{\pi} \sqrt{\alpha} \Delta k^{3 / 2}}\left(2 \pi \Delta k \quad \operatorname{erf}\left[\frac{\sqrt{\alpha}}{2 \sqrt{\Delta k}}\right] \operatorname{erfi}\left[\frac{\sqrt{\alpha}}{2 \sqrt{\Delta k}}\right]-\alpha F\left[(1,1),\left(\frac{3}{2}, 2\right),-\frac{\alpha}{4 \Delta k}\right]\right. \\
& \left.\left.-2 \pi \Delta k \quad \operatorname{erf}\left[\frac{\sqrt{\alpha}(1+\Delta k)}{2 \sqrt{\Delta k}}\right] \operatorname{erfi}\left[\frac{\sqrt{\alpha}}{2 \sqrt{\Delta k}}\right]+\alpha(1+\Delta k)^{2} F\left[(1,1),\left(\frac{3}{2}, 2\right),-\frac{\alpha(1+\Delta k)^{2}}{4 \Delta k}\right]\right)\right\}
\end{aligned}
$$

Así como lo hicimos en el proceso de 1 a 2 escribimos el valor del trabajo adimensional $\langle W\rangle^{*}=\frac{\varepsilon\langle W\rangle}{k_{1}}$

$$
\begin{aligned}
\left\langle W_{3 \rightarrow 4}\right\rangle^{*} & =-\frac{\sqrt{\pi} \Delta k}{4 \sqrt{\alpha} \sqrt{\Delta k}}\left\{e^{\frac{\alpha}{4 \Delta k}}\left(\operatorname{erf}\left[\frac{\sqrt{\alpha}}{2 \sqrt{\Delta k}}\right]-\operatorname{erf}\left[\frac{\sqrt{\alpha}(1+\Delta k)}{2 \sqrt{\Delta k}}\right]\right)\right. \\
& -\frac{2 \alpha}{8 \sqrt{\pi} \sqrt{\alpha} \Delta k^{3 / 2}}\left(2 \pi \Delta k \quad \operatorname{erf}\left[\frac{\sqrt{\alpha}}{2 \sqrt{\Delta k}}\right] \operatorname{erfi}\left[\frac{\sqrt{\alpha}}{2 \sqrt{\Delta k}}\right]-\alpha F\left[(1,1),\left(\frac{3}{2}, 2\right),-\frac{\alpha}{4 \Delta k}\right]\right. \\
& \left.\left.-2 \pi \Delta k \quad \operatorname{erf}\left[\frac{\sqrt{\alpha}(1+\Delta k)}{2 \sqrt{\Delta k}}\right] \operatorname{erfi}\left[\frac{\sqrt{\alpha}}{2 \sqrt{\Delta k}}\right]+\alpha(1+\Delta k)^{2} F\left[(1,1),\left(\frac{3}{2}, 2\right),-\frac{\alpha(1+\Delta k)^{2}}{4 \Delta k}\right]\right)\right\}
\end{aligned}
$$

Así el trabajo solo depende del cambio de la rigidez del potencial $\Delta k$, del cambio en la intensidad del ruido $\Delta q$ y de la variable adimensional $\alpha=\frac{k_{1} \tau}{m \gamma}$. Damos los mismo valores adimensionales para encontrar el límite y graficar con respecto a $\alpha$. El límite cuando $\alpha$ tiende a infinito

$$
\lim _{\alpha \rightarrow \infty}\left\langle W_{3 \rightarrow 4}\right\rangle^{*}=\frac{\log [2]}{4}
$$

La gráfica se muestra en el capítulo 7 


\section{E.4. 4 a 1}

Este proceso es un cambio en la intensidad del ruido manteniendo la rigidez del potencial constante,

$$
\frac{d k(t)}{d t}=0
$$

ya que el trabajo depende del cambio en el potencial, durante este proceso no hay trabajo

$$
\langle W(x, t)\rangle=0
$$

Durante el ciclo hay dos procesos en los que existe trabajo, el signo nos indica si el trabajo está siendo ejercido sobre la partícula o si la partícula está haciendo trabajo sobre el sistema. En nuestra convención de signos, el positivo indica que se está haciendo trabajo sobre la partícula y el negativo indica lo contrario. En el primer proceso del ciclo cuando se disminuye la rigidez del potencial para limitar el movimiento el trabajo es negativo, es decir al reducir la rigidez del potencial existe un trabajo que realiza la partícula sobre el sistema. En el proceso de 3 a 4 cuando se aumenta la rigidez del potencial se tiene un trabajo positivo que se está ejerciendo sobre el sistema.

Graficando los trabajos del ciclo juntos podemos notar que el valor absoluto del trabajo ejercido sobre el sistema (el que es positivo) siempre es menor que el valor absoluto del trabajo generado (el que es negativo). Por lo tanto, hay trabajo generado. Siguiendo los postulados de la mecánica clásica, para que esto ocurra debe de haber calor absorbido por el sistema. 



\section{Apéndice $\mathrm{F}$}

\section{Entropía en el ciclo}

La entropía sobre una trayectoria está dada por

$$
s(t)=-\ln w(x(t), t)
$$

donde

$$
w(x(t), t)=\sqrt{\frac{\epsilon(t)}{\pi}} e^{-\epsilon(t) x^{2}}
$$

tiene una forma Gaussiana.

Entonces para determinar la entropía sobre una trayectoria reescribirmos el logaritmo de $w$ usando las propiedades de los logaritmos

$$
\begin{array}{r}
\operatorname{Ln}[w(x(t), t)]=\operatorname{Ln}\left[\sqrt{\frac{\epsilon(t)}{\pi}} e^{-\epsilon(t) x^{2}}\right]=\operatorname{Ln}\left[\sqrt{\frac{\epsilon(t)}{\pi}}\right]+\operatorname{Ln}\left[e^{-\epsilon(t) x^{2}}\right] \\
=\operatorname{Ln}\left[\sqrt{\frac{\epsilon(t)}{\pi}}\right]-\epsilon(t) x^{2}=\frac{\operatorname{Ln}\left[\frac{\epsilon(t)}{\pi}\right]}{2}-\epsilon(t) x^{2}
\end{array}
$$

de esta manera la entropía está dada por

$$
s(t)=-\frac{\operatorname{Ln}\left[\frac{\epsilon(t)}{\pi}\right]}{2}+\epsilon(t) x^{2}
$$

$\mathrm{Y}$ el promedio sobre muchas realizaciones es

$$
\langle s(t)\rangle=-\frac{1}{2} \operatorname{Ln}\left[\frac{\epsilon(t)}{\pi}\right]+\epsilon(t)\left\langle x^{2}\right\rangle
$$

Pero el promedio de $x^{2}$ es

$$
\left\langle x^{2}\right\rangle=\frac{1}{2 \epsilon(t)}
$$

Por lo tanto

$$
\langle s(t)\rangle=-\frac{1}{2} \operatorname{Ln}\left[\frac{\epsilon(t)}{\pi}\right]+\epsilon(t) \frac{1}{2 \epsilon(t)}=-\frac{1}{2} \operatorname{Ln}\left[\frac{\epsilon(t)}{\pi}\right]+\frac{1}{2}
$$

El cambio de entropía que se genera durante el proceso está dado por

$$
\begin{aligned}
& \Delta\langle s(t)\rangle=\left\langle s\left(t_{f}\right)\right\rangle-\left\langle s\left(t_{o}\right)\right\rangle \\
& =-\frac{1}{2} \operatorname{Ln}\left[\frac{\epsilon\left(t_{f}\right)}{\pi}\right]+\frac{1}{2}+\frac{1}{2} \operatorname{Ln}\left[\frac{\epsilon\left(t_{o}\right)}{\pi}\right]-\frac{1}{2} \\
& =-\frac{1}{2} \operatorname{Ln}\left[\frac{\epsilon\left(t_{f}\right)}{\pi}\right]+\frac{1}{2} \operatorname{Ln}\left[\frac{\epsilon\left(t_{o}\right)}{\pi}\right]=\frac{1}{2}\left(-\operatorname{Ln}\left[\frac{\epsilon\left(t_{f}\right)}{\pi}\right]+\operatorname{Ln}\left[\frac{\epsilon\left(t_{o}\right)}{\pi}\right]\right) \\
& =\frac{1}{2}\left(\operatorname{Ln}\left[\frac{\frac{\epsilon\left(t_{o}\right)}{\pi}}{\frac{\epsilon\left(t_{f}\right)}{\pi}}\right]\right)=\frac{1}{2}\left(\operatorname{Ln}\left[\frac{\epsilon\left(t_{o}\right)}{\epsilon\left(t_{f}\right)}\right]\right)
\end{aligned}
$$

Veremos el cambio de entropía durante cada proceso del ciclo 


\section{F.1. 1 a 2}

En el primer proceso se toma como condición inicial que el sistema se encuentra en equilibrio en este caso $\epsilon\left(t_{0}\right)=\frac{\gamma k_{2}}{m q_{2}}$, Tomando eso en cuenta podemos escribir la solución como

$$
\begin{aligned}
\frac{1}{\epsilon_{1 \rightarrow 2}(t)} & =\frac{1}{\epsilon\left(t_{0}\right)}\left(e^{\frac{2 \alpha t}{\tau}\left[2 \Delta k \frac{t}{\tau}-(\Delta k+1)\right]}\right. \\
& \left.+\frac{\sqrt{\alpha}(1+\Delta k) \sqrt{\pi} e^{\frac{\alpha}{4 \Delta k}\left(-4 \Delta k \frac{t}{\tau}+(\Delta k+1)\right.}}{2 \sqrt{\Delta k}}\left(\operatorname{erf}\left[\frac{\sqrt{\alpha}(\Delta k+1)}{2 \sqrt{\Delta k}}\right]-\operatorname{erf}\left[\frac{\sqrt{\alpha}\left(-4 \Delta k \frac{t}{\tau}+(\Delta k+1)\right)}{2 \sqrt{\Delta k}}\right]\right)\right)
\end{aligned}
$$

El proceso de 1 a 2 se lleva a cabo en un tiempo de $t_{o}=0$ a $t_{f}=\frac{\tau}{4}$ debemos de evaluar la solución en el tiempo inicial y en el tiempo final

$$
\begin{aligned}
\frac{1}{\epsilon_{1 \rightarrow 2}(0)} & =\frac{1}{\epsilon\left(t_{0}\right)} \\
& \text { cuando evaluamos en el tiempo final } \\
\frac{1}{\epsilon_{1 \rightarrow 2}\left(\frac{\tau}{4}\right)} & =\frac{1}{\epsilon\left(t_{0}\right)}\left(e^{\frac{2 \alpha \frac{\tau}{4}}{\tau}\left[2 \Delta k \frac{\frac{\tau}{4}}{\tau}-(\Delta k+1)\right]}\right. \\
& \left.+\frac{\sqrt{\alpha}(1+\Delta k) \sqrt{\pi} e^{\frac{\alpha}{4 \Delta k}\left(-4 \Delta k \frac{\tau}{\tau}+(\Delta k+1)\right.}}{2 \sqrt{\Delta k}}\left(\operatorname{erf}\left[\frac{\sqrt{\alpha}(\Delta k+1)}{2 \sqrt{\Delta k}}\right]-\operatorname{erf}\left[\frac{\sqrt{\alpha}\left(-4 \Delta k \frac{\tau}{\tau}+(\Delta k+1)\right)}{2 \sqrt{\Delta k}}\right]\right)\right) \\
& =\frac{1}{\epsilon\left(t_{0}\right)}\left(e^{\frac{\alpha}{2}\left[\Delta k \frac{1}{2}-(\Delta k+1)\right]}\right. \\
& \left.+\frac{\sqrt{\alpha}(1+\Delta k) \sqrt{\pi} e^{\frac{\alpha}{4 \Delta k}}}{2 \sqrt{\Delta k}}\left(\operatorname{erf}\left[\frac{\sqrt{\alpha}(\Delta k+1)}{2 \sqrt{\Delta k}}\right]-\operatorname{erf}\left[\frac{\sqrt{\alpha}}{2 \sqrt{\Delta k}}\right]\right)\right)
\end{aligned}
$$

Al hacer los cálculos para encontrar el promedio de la entropía encontramos

$$
\begin{aligned}
\Delta\langle s\rangle_{1 \rightarrow 2} & =\frac{1}{2} \operatorname{Ln}\left[\frac{\epsilon\left(t_{o}\right)}{\epsilon\left(t_{f}\right)}\right] \\
& =\frac{1}{2} \operatorname{Ln}\left[e^{\frac{\alpha}{2}\left[\Delta k \frac{1}{2}-(\Delta k+1)\right]}+\frac{\sqrt{\alpha}(1+\Delta k) \sqrt{\pi} e^{\frac{\alpha}{4 \Delta k}}}{2 \sqrt{\Delta k}}\left(\operatorname{erf}\left[\frac{\sqrt{\alpha}(\Delta k+1)}{2 \sqrt{\Delta k}}\right]-\operatorname{erf}\left[\frac{\sqrt{\alpha}}{2 \sqrt{\Delta k}}\right]\right)\right]
\end{aligned}
$$

$\mathrm{Al}$ dar valor a $\Delta k=1$ y $\Delta q=1$ entonces podemos ver el comportamiento de la eficiencia mientras $\alpha$ es grande. El límite cuando $\alpha$ tiende a infinito es

$$
\lim _{\alpha \rightarrow \infty} \Delta\langle s\rangle_{1 \rightarrow 2}=\frac{\log [2]}{2}
$$

y graficamente

\section{F.2. 2 a 3}

$$
\frac{1}{\epsilon_{2 \rightarrow 3}(t)}=-\frac{1}{\epsilon\left(t_{0}\right)} e^{-\frac{2 \alpha\left(t-\frac{\tau}{4}\right)}{\tau}}-\frac{q_{1}}{\gamma^{2} \alpha^{2}}\left(4 t \alpha \Delta q+\tau\left(2 \Delta q\left[-1+e^{-\frac{2 \alpha}{\tau}(t-\tau / 4)}\right]+\alpha\left[-1-2 \Delta q+e^{-\frac{2 \alpha}{\tau}(t-\tau / 4)}(1+\Delta q)\right]\right)\right)
$$

El proceso de 2 a 3 se lleva a cabo durante el tiempo $t_{0}=\frac{\tau}{4}$ y $\frac{2 \tau}{4}$ evaluamos la solución en los dos tiempos.

$$
\begin{aligned}
& \frac{1}{\epsilon_{2 \rightarrow 3}\left(\frac{\tau}{4}\right)}=\frac{1}{\epsilon\left(t_{0}\right)} \\
& \text { y para el tiempo final tenemos } \\
& \frac{1}{\epsilon_{2 \rightarrow 3}\left(\frac{2 \tau}{4}\right)}=-\frac{1}{\epsilon\left(t_{0}\right)} e^{-\frac{\alpha}{2}}-\frac{q_{1} m}{k_{1} \tau \gamma \alpha}\left(2 \tau \alpha \Delta q+\tau\left(2 \Delta q\left[-1+e^{-\frac{2 \alpha}{\tau}\left(\frac{\tau}{4}\right)}\right]+\alpha\left[-1-2 \Delta q+e^{-\frac{2 \alpha}{\tau}\left(\frac{\tau}{4}\right)}(1+\Delta q)\right]\right)\right) \\
&=-\frac{1}{\epsilon\left(t_{0}\right)} e^{-\frac{\alpha}{2}}-\frac{q_{1} m}{k_{1} \gamma \alpha}\left(2 \Delta q\left[-1+e^{-\frac{\alpha}{2}}\right]-\alpha+\alpha e^{-\frac{\alpha}{2}}(1+\Delta q)\right) \\
&=-\frac{1}{\epsilon\left(t_{0}\right)} e^{-\frac{\alpha}{2}}-\frac{1}{\epsilon_{e q} \alpha(1+\Delta q)}\left(2 \Delta q\left[-1+e^{-\frac{\alpha}{2}}\right]-\alpha+\alpha e^{-\frac{\alpha}{2}}(1+\Delta q)\right)
\end{aligned}
$$


El cambio de entropía durante el proceso es el siguiente

$$
\Delta\langle s\rangle_{2 \rightarrow 3}=\frac{1}{2} \operatorname{Ln}\left[\epsilon\left(t_{0}\right)\left(-\frac{1}{\epsilon\left(t_{0}\right)} e^{-\frac{\alpha}{2}}-\frac{1}{\epsilon_{e q} \alpha(1+\Delta q)}\left(2 \Delta q\left[-1+e^{-\frac{\alpha}{2}}\right]-\alpha+\alpha e^{-\frac{\alpha}{2}}(1+\Delta q)\right)\right)\right]
$$

Cuando $\alpha$ tiende a infinito entonces

$$
\lim _{\alpha \rightarrow \infty} \Delta\langle s\rangle_{2 \rightarrow 3}=\frac{1}{2} \operatorname{Ln}\left[\frac{\epsilon\left(t_{0}\right)}{\epsilon_{e q}(1+\Delta q)}\right]
$$

Tomando el caso en que $\epsilon\left(t_{0}\right)=\epsilon_{e q}$ podemos graficar si damos el valor $\Delta k=1$ y $\Delta q=1$

\section{F.3. 3 a 4}

$\frac{1}{\epsilon_{3 \rightarrow 4}(t)}=\frac{1}{\epsilon\left(t_{0}\right)} e^{-\frac{\alpha(2 t-\tau)(2 t \Delta k+\tau-\Delta k \tau)}{\tau^{2}}}+\frac{2 q 1 \sqrt{\pi} \tau e^{-\frac{\alpha}{4 \Delta k \tau^{2}}(4 \Delta k t-2 \Delta k \tau+\tau)^{2}}}{4 \gamma^{2} \sqrt{\alpha \Delta k}}\left[\operatorname{erfi}\left(\frac{\sqrt{\alpha}}{2 \sqrt{\Delta k}}\right)-\operatorname{erfi}\left(\frac{\sqrt{\alpha}(4 t \Delta k+\tau-2 \Delta k \tau)}{2 \tau \sqrt{\Delta k}}\right)\right]$

Es lo mismo en este caso el tiempo va de $t_{0}=\frac{2 \tau}{4}$ a $t_{f}=\frac{3 \tau}{4}$ al evaluar la solución en los tiempos llegamos a

$$
\frac{1}{\epsilon_{3 \rightarrow 4}\left(\frac{2 \tau}{4}\right)}=\frac{1}{\epsilon\left(t_{0}\right)}
$$

y para el tiempo final

$$
\begin{aligned}
& \frac{1}{\epsilon_{3 \rightarrow 4}\left(\frac{3 \tau}{4}\right)}=\frac{1}{\epsilon\left(t_{0}\right)} e^{-\frac{\alpha\left(2 \frac{3 \tau}{4}-\tau\right)\left(2 \frac{3 \tau}{4} \Delta k+\tau-\Delta k \tau\right)}{\tau^{2}}}+\frac{2 q_{1} \sqrt{\pi} \tau e^{-\frac{\alpha}{4 \Delta k \tau^{2}}\left(4 \Delta k \frac{3 \tau}{4}-2 \Delta k \tau+\tau\right)^{2}}}{4 \gamma^{2} \sqrt{\alpha \Delta k}}\left[\operatorname{erfi}\left(\frac{\sqrt{\alpha}}{2 \sqrt{\Delta k}}\right)-\operatorname{erfi}\left(\frac{\sqrt{\alpha}\left(4 \frac{3 \tau}{4} \Delta k+\tau-2 \Delta k \tau\right)}{2 \tau \sqrt{\Delta k}}\right)\right] \\
& \frac{1}{\epsilon_{3 \rightarrow 4}\left(\frac{3 \tau}{4}\right)}=\frac{1}{\epsilon\left(t_{0}\right)} e^{-\frac{\alpha\left(\frac{\Delta k}{2}+1\right)}{2}}+\frac{2 q_{1} \sqrt{\pi} \tau e^{-\frac{\alpha}{4 \Delta k}(\Delta k+1)^{2}}}{4 \gamma^{2} \sqrt{\alpha \Delta k}}\left[\operatorname{erfi}\left(\frac{\sqrt{\alpha}}{2 \sqrt{\Delta k}}\right)-\operatorname{erfi}\left(\frac{\sqrt{\alpha}(\Delta k+1)}{2 \sqrt{\Delta k}}\right)\right] \\
& =\frac{1}{\epsilon\left(t_{0}\right)} e^{-\frac{\alpha\left(\frac{\Delta k}{2}+1\right)}{2}}+\frac{\sqrt{\alpha} \sqrt{\pi} e^{-\frac{\alpha}{4 \Delta k}(\Delta k+1)^{2}}}{2 \epsilon_{e q} \sqrt{\Delta k}}\left[\operatorname{erfi}\left(\frac{\sqrt{\alpha}}{2 \sqrt{\Delta k}}\right)-\operatorname{erfi}\left(\frac{\sqrt{\alpha}(\Delta k+1)}{2 \sqrt{\Delta k}}\right)\right]
\end{aligned}
$$

Tomando esto en cuenta el cambio de entropía durante el proceso es el siguiente

$$
\begin{aligned}
\Delta\langle s\rangle_{3 \rightarrow 4} & =\frac{1}{2} \operatorname{Ln}\left[\frac{\epsilon\left(t_{o}\right)}{\epsilon\left(t_{f}\right)}\right] \\
& =\frac{1}{2} \operatorname{Ln}\left[\epsilon\left(t_{0}\right)\left(\frac{1}{\epsilon\left(t_{0}\right)} e^{-\frac{\alpha\left(\frac{\Delta k}{2}+1\right)}{2}}+\frac{\sqrt{\alpha} \sqrt{\pi} e^{-\frac{\alpha}{4 \Delta k}(\Delta k+1)^{2}}}{2 \epsilon_{e q} \sqrt{\Delta k}}\left[\operatorname{erfi}\left(\frac{\sqrt{\alpha}}{2 \sqrt{\Delta k}}\right)-\operatorname{erfi}\left(\frac{\sqrt{\alpha}(\Delta k+1)}{2 \sqrt{\Delta k}}\right)\right]\right)\right]
\end{aligned}
$$

Cuando $\epsilon\left(t_{0}\right)=\epsilon_{e q}$ y damos valores numéricos a $\Delta k_{1}=1$ y $\Delta q_{1}=1$ encontramos que el límite cuando $\alpha$ tiende a infinito es

$$
\lim _{\alpha \rightarrow \infty} \Delta\langle s\rangle_{3 \rightarrow 4}=-\frac{\log [2]}{2}
$$

y al graficar el comportamiento de este encontramos su decaimiento al valor límite

\section{F.4. $\quad 4$ a 1}

$$
\begin{aligned}
\frac{1}{\epsilon_{4 \rightarrow 1}(t)} & =\frac{e^{-\frac{2 \alpha(1+\Delta k)\left(t-\frac{3 \tau}{4}\right)}{\tau}}}{\epsilon\left(t_{0}\right)}+ \\
& \frac{q_{1}}{\gamma^{2} \alpha^{2}(1+\Delta k)^{2}}\left[4 t \alpha \Delta q(1+\Delta k)-\tau\left(-2 \Delta q\left(-1+e^{\frac{2 \alpha(1+\Delta k)\left(-t+\frac{3 \tau}{4}\right)}{\tau}}\right)+\alpha(1+\Delta k)\left(-1+e^{\frac{2 \alpha(1+\Delta k)\left(-t+\frac{3 \tau}{4}\right)}{\tau}}+3 \Delta q\right)\right)\right]
\end{aligned}
$$

en este caso el tiempo va de $t_{0}=\frac{3 \tau}{4}$ a $t_{f}=\tau$ al evaluar la solución en los tiempos llegamos a

$$
\frac{1}{\epsilon_{4 \rightarrow 1}(t)}=\frac{1}{\epsilon\left(t_{0}\right)}
$$


y para el tiempo final tenemos

$$
\begin{aligned}
\frac{1}{\epsilon_{4 \rightarrow 1}(t)} & =\frac{e^{-\frac{2 \alpha(1+\Delta k)\left(\frac{\tau}{4}\right)}{\tau}}+}{\epsilon\left(t_{0}\right)}+ \\
& \frac{q_{1}}{\gamma^{2} \alpha^{2}(1+\Delta k)^{2}}\left[4 \tau \alpha \Delta q(1+\Delta k)-\tau\left(-2 \Delta q\left(-1+e^{\frac{2 \alpha(1+\Delta k)\left(-\frac{\tau}{4}\right)}{\tau}}\right)+\alpha(1+\Delta k)\left(-1+e^{\left.\left.\left.\frac{2 \alpha(1+\Delta k)\left(-\frac{\tau}{4}\right)}{\tau}+3 \Delta q\right)\right)\right]}\right.\right.\right. \\
& =\frac{e^{-(1+\Delta k)\left(\frac{\alpha}{2}\right)}}{\epsilon\left(t_{0}\right)}+ \\
& \frac{q_{1} \tau}{\gamma^{2} \alpha^{2}(1+\Delta k)^{2}}\left[4 \alpha \Delta q(1+\Delta k)-\left(-2 \Delta q\left(-1+e^{(1+\Delta k)\left(-\frac{\alpha}{2}\right)}\right)+\alpha(1+\Delta k)\left(-1+e^{(1+\Delta k)\left(-\frac{\alpha}{2}\right)}+3 \Delta q\right)\right)\right] \\
& =\frac{e^{-(1+\Delta k)\left(\frac{\alpha}{2}\right)}}{\epsilon\left(t_{0}\right)}+ \\
& \frac{1}{\epsilon_{e q} \alpha(1+\Delta k)}\left[4 \alpha \Delta q(1+\Delta k)-\left(-2 \Delta q\left(-1+e^{(1+\Delta k)\left(-\frac{\alpha}{2}\right)}\right)+\alpha(1+\Delta k)\left(-1+e^{(1+\Delta k)\left(-\frac{\alpha}{2}\right)}+3 \Delta q\right)\right)\right] \quad(\mathrm{F} .34)
\end{aligned}
$$

Tomando esto en cuenta podemos escribir la diferencia de entropía en el proceso

$$
\begin{aligned}
\Delta\langle s\rangle_{4 \rightarrow 1} & =\frac{1}{2} \operatorname{Ln}\left[\frac{\epsilon\left(t_{o}\right)}{\epsilon\left(t_{f}\right)}\right] \\
& =\frac{1}{2} \operatorname{Ln}\left[\epsilon ( t _ { 0 } ) \left(\frac{e^{-(1+\Delta k)\left(\frac{\alpha}{2}\right)}}{\epsilon\left(t_{0}\right)}+\right.\right. \\
& \left.\left.\frac{1}{\epsilon_{e q} \alpha(1+\Delta k)}\left[4 \alpha \Delta q(1+\Delta k)-\left(-2 \Delta q\left(-1+e^{(1+\Delta k)\left(-\frac{\alpha}{2}\right)}\right)+\alpha(1+\Delta k)\left(-1+e^{(1+\Delta k)\left(-\frac{\alpha}{2}\right)}+3 \Delta q\right)\right)\right]\right)\right]
\end{aligned}
$$

En el límite cuando $\alpha$ tiende a infinito encontramos

$$
\lim _{\alpha \rightarrow \infty} \Delta\langle s\rangle_{4 \rightarrow 1}=\frac{1}{2} \operatorname{Ln}\left[\frac{\epsilon\left(t_{0}\right)}{\epsilon_{e q}}(\Delta q+1)\right]
$$

En el caso en el que $\epsilon\left(t_{0}\right)=\epsilon_{e q}$ y damos valores numéricos a $\Delta k_{1}=1$ y $\Delta q_{1}=1$ encontramos el comportamiento de la eficiencia variando $\alpha$

\section{F.4.1. Análisis ciclo completo}

Durante la realización de los 4 procesos vemos que la solución tiende al valor en equilibrio, sinembargo nunca llega a este estado, en el límite la máquina se comporta como un ciclo ideal en elq ue el total de la variación de la entropía es nula, solo en este caso el ciclo es reversible. 


\section{Bibliografía}

[1] B.-Q. Ai, L. Wang, and L.-G. Liu. Brownian micro-engines and refrigerators in a spatially periodic temperature field: Heat flow and performances. Physics Letters A, 352:286-290, April 2006.

[2] D. Arold, A. Dechant, and E. Lutz. Heat leakage in overdamped harmonic systems. Physical Review E, 97:022131, February 2018.

[3] J. D. Badjic, C. M. Ronconi, et al. Operating molecular elevators. Journal of the American Chemical Society Articles, 128:1489-1499, January 2006.

[4] L. Benguigui. The different paths to entropy. European Journal of Physics, 34:303-321, January 2013.

[5] V. Blickle and C. Bechinger. Realization of a micrometre-sized stochastic heat engine. Nature Physics Letters, 8:143-146, 2012.

[6] E. Braun. Un movimiento en zigzag. Fondo de Cultura Económica, 1 edition, 2011.

[7] R. Brown. A brief account of microscopical observations made in the months of june, july, and august, 1827, on the particles contained in the pollen of plants; and on the general existence of active molecules in organic and inorganic bodies. Philosophical Magazine N. S. 4, 4, September 1828.

[8] M. Büttiker. Transport as a consequence of state-dependent diffusion. Zeitschrift für Physik B Condendes Matter, 68:161-167, June 1987.

[9] F. L. Curzon and B. Alborn. Efficiency of a carnot engine at maximum power output. American Journal of physics, 43:22-24, January 1975.

[10] A. Einstein. Investigation of the theory of the Brownian Movement. Dover Publications, 1 edition, 1956.

[11] M. Esposito, R. Kawai, K. Lindenberg, et al. Efficiency at maximum power of low-dissipation carnot engines. Physical Review Letters, 105:150603, October 2010 .

[12] M. Esposito, K. Lindenberg, and C. van den Broeck. Universality of efficienc at maximum power. Physical Review Letters, 102:130602, April 2009.

[13] L. García-Colín. Introducción a la termodinámica clásica. Trillas, 1995.

[14] L. García-Colín. De la máquina de vapor al cero absoluto. Fondo de cultura económica, 3 edition, 2003. 
[15] T. L. Hill. Thermodynamics of small systems. Dover Publications, 1 edition, 1994.

[16] K. Itô. Stochastic integral. Proceedings of the Imperial Academy, 20:519-524, Oct 1944.

[17] J. S. Kim and S. S. Lee. Scattering of laser beams and the optical potential well for a homogeneous sphere. Journal of the Optical Society of America, 73:303-312, 1983.

[18] R. Landauer. Motion out of noisy states. Journal of Statistical Physics, 53:233-248, October 1988.

[19] P. Langevin. Sur la theorie du mouvement brownien. 1908.

[20] I. Martínez, E. Roldán, et al. Brownian carnot engine. Nature Physics Letters, 12:67-70, 2016.

[21] I. A. Martínez, Édgar Roldán, et al. Adiabatic processes realized with a trapped brownuan particle. Physical Review Letters, 114:120601, March 2015.

[22] N. Méndez. Construcción y calibración de un sistema de pinzas ópticas. Master's thesis, Universidad Autónoma Metropolitana, 2014.

[23] E. Nelson. Dynamical Theories of Brownian Motion. Princeton University Press, 2 edition, 2001.

[24] J. Perrin. Brownian movement and molecular reality. Dover Phoenix Edition, 1 edition, 2013.

[25] S. Rana, P. S. Pal, et al. Single- particle stochastic heat engine. Physical Review E, 90:042146, October 2014.

[26] A. C. Richardson, S. N. S. Reihani, and L. Oddershede. Non-harmonic potential of a single beam optical trap. Optics Express, 16:15709-15717, 2008.

[27] H. Risken. The Fokker-Planck Equation. Springer-Verlag, 2 edition, 1988.

[28] R. Salcedo. Las ventajas de ser débil: Premio nobel de química 2016. Educación química, 28:59-61, January 2017.

[29] T. Schmiedl and U. Seifert. Efficiency at maximum power: An analytically solvable model for stochastic heat engines. Europhysics Letters, 81, January 2008 .

[30] U. Seifert. Entropy production along a stochastic trajectory and an integral fluctuation theorem. Physical Review Letters, 95:040602, July 2005.

[31] U. Seifert. Stochastic thermodynamics: Principles and perspectives. The European Physical Journal B, 64:423-431, January 2008.

[32] U. Seifert. Stochastic thermodynamics, fluctuation theorems and molecular machines. Reports on Progress in Physics, 75:126001, November 2012.

[33] K. Sekimoto. Langevin equation and thermodynamics. Progress of Theoretical Physics Supplement, 130:17-27, January 1998. 
[34] K. Sekimoto. Stochastic Energetics. Lecture Notes in Physics. Springer, 2010 .

[35] S. P. Sethi and J. P. Lehoczky. A comparison of the ito and stratonovich formulations of problems in finance. Journal of Economic Dynamics and Control, 3:343-356, 1981.

[36] R. L. Stratonovich. A new representation for stochastic integrals and equations. SIAM Journal on Control, 4:362-371, 1966.

[37] The Nobel Commitee for physics. Goundbreaking inventions in laser physics. The royal swedish academy of sciences, October 2018.

[38] D. G. Thombare and S. K. Verma. Technological development in the stirling cycle engines. Renewable and Sustainable Energy Reviews, 12:1-38, January 2008 .

[39] S. A. Torres. Atrapamiento y manipulación óptica para aplicaciones biológicas. PhD thesis, Instituto Nacional de Astrofísica, Óptica y Electrónica, 2016 .

[40] Z. C. Tu. Stochastic heat engine with the consideration of inertial effects and shortcuts to adiabaticity. Physical Review E, 89:052148, May 2014.

[41] J. Valero, N. Pal, et al. A bio-hybrid dna rotor-stator nanoengine that moves along predefined tracks. Nature Nanotechnoogy, 13:469-503, April 2018.

[42] N. van Kampen. Relative stability in nonuniform temperature. IBM Journal of Research and Development, 32:107-111, January 1988.

[43] M. von Smoluchowski. Annalen der Physik, 21:756, 1906. 
Ciclo de stirling para una particula Browniana
En la Ciudad de México, se presentaron a las 11:00 horas del día 14 del mes de noviembre del año 2019 en la Unidad Iztapalapa de la Universidad Autónoma Metropolitana, los suscritos miembros del jurado:

DRA, ROSA MARIA VELASCO BELMONT

DRA. LORENA ROMERO SALAZAR

DR. JOSE INES JIMENEZ AQUINO

Bajo la presidencia de la primera y con carácter de Secretario el último, se reunieron para proceder al Examen de Grado cuya denominación aparece al margen, para la obtención del grado de:

MAESTRA EN CIENCIAS (FISICA)

DE: ANA ELISA ZAMUdIO MURILLO

$y$ de acuerdo con el artículo 78 fracción III del Reglamento de Estudios Superiores de la Universidad Autónoma Metropolitana, los miembros del jurado resolvieron:

\section{APROBAR}

Acto continuo, la presidenta del jurado comunicó a la interesada el resultado de la evaluación $y$, en caso aprobatorio, le fue tomada la protesta. 